Copyright

by

Rustem Ospanov

2008 
The Dissertation Committee for Rustem Ospanov

certifies that this is the approved version of the following dissertation:

\title{
A measurement of muon neutrino disappearance with the MINOS detectors and NuMI beam
}

\author{
Committee:
}

Karol Lang, Supervisor

Duane Dicus

Karl Gebhardt

Sacha Kopp

Jack Ritchie 


\title{
A measurement of muon neutrino disappearance with the MINOS detectors and NuMI beam
}

\author{
by
}

Rustem Ospanov, B.S., M.A.

\author{
DISSERTATION \\ Presented to the Faculty of the Graduate School of \\ The University of Texas at Austin \\ in Partial Fulfillment \\ of the Requirements \\ for the Degree of \\ DOCTOR OF PHILOSOPHY
}

THE UNIVERSITY OF TEXAS AT AUSTIN

December 2008 
Dedicated to my family. 


\title{
A measurement of muon neutrino disappearance with the MINOS detectors and NuMI beam
}

\author{
Publication No. \\ Rustem Ospanov, Ph.D. \\ The University of Texas at Austin, 2008 \\ Supervisor: Karol Lang
}

MINOS is a long-baseline two-detector neutrino oscillation experiment that uses a high intensity muon neutrino beam to investigate the phenomena of neutrino oscillations. The neutrino beam is produced by the NuMI facility at Fermilab, Batavia, Illinois, and is observed at near and far detectors placed $734 \mathrm{~km}$ apart. The neutrino interactions in the near detector are used to measure the initial muon neutrino flux. The vast majority of neutrinos travel through the near detector and Earth matter without interactions. A fraction of muon neutrinos oscillate into other flavors resulting in the disappearance of muon neutrinos at the far detector. This thesis presents a measurement of the muon neutrino oscillation parameters in the framework of the two-neutrino oscillation hypothesis. 


\section{Table of Contents}

Abstract $\quad$ v

Chapter 1. Introduction to neutrino physics 1

1.1 A brief history of neutrinos . . . . . . . . . . . . . . . 1

1.2 Theory of neutrino oscillations . . . . . . . . . . . . 6

1.3 Experimental evidence for neutrino oscillations . . . . . . . . 12

1.3.1 Electron neutrinos from the sun . . . . . . . . . 13

1.3.2 Electron anti-neutrinos from nuclear reactors . . . . . . 19

1.3.3 Atmospheric neutrinos . . . . . . . . . . . . . 21

1.3.4 Man-made muon neutrino beams . . . . . . . . . . 23

1.4 Summary of neutrino oscillations . . . . . . . . . . . . . 25

1.5 Outline of the dissertation . . . . . . . . . . . 26

Chapter 2. NuMI facility and MINOS detectors 30

2.1 Neutrino beams . . . . . . . . . . . . . . . . . . . . . 30

2.2 NuMI facility . . . . . . . . . . . . . . . . . 31

2.3 MINOS detectors ................... . . . 37

2.4 Detector calibration ................ . . 46

Chapter 3. Neutrino interactions in the MINOS detectors 53

3.1 Event reconstruction ................. 54

3.2 Charged-current muon neutrino interactions . . . . . . . . 60

3.3 Selection of QES, RES, and DIS events . . . . . . . . . . . . 69

3.4 Energy reconstruction in QES, RES, and DIS events . . . . . . 79

3.5 Monte-Carlo simulation . . . . . . . . . . . . . . 83

3.6 Study of near detector hits in the data and MC simulation . . 87

3.7 Summary . . . . . . . . . . . . . . . . 96 
$\begin{array}{lll}\text { Chapter 4. } & \text { Identification of muon tracks } & 98\end{array}$

4.1 Reconstructed muon and non-muon tracks . . . . . . . . . . . 100

4.2 Improving sensitivity to muon tracks . . . . . . . . . . . . . . 101

4.3 Track based muon identification variables . . . . . . . . . . . . 104

4.3.1 Number of track scintillator planes . . . . . . . . . . . 106

4.3.2 Mean pulse height of track hits . . . . . . . . . . . . 108

4.3.3 Signal fluctuation . . . . . . . . . . . . . . . . . . . . . . . . . . . . . . . . . 111

4.3.4 Transverse track profile . . . . . . . . . . . . . . 111

4.4 Event classification with the k-nearest neighbor algorithm . . . 115

4.5 Identification of muon neutrino charged-current events . . . . . 120

4.6 A study of event selection for several beam configurations . . . 123

4.7 Comparison of muon tracks for data and MC . . . . . . . . . . 124

4.8 Summary . . . . . . . . . . . . . . . . . . . 131

$\begin{array}{lll}\text { Chapter 5. } & \text { Measurement of muon charge sign } & 133\end{array}$

5.1 Charge-sign measurement using fitting algorithm . . . . . . . . 134

5.2 New method for charge-sign measurement . . . . . . . . . . . . 136

5.3 Charge-sign for non-muon tracks . . . . . . . . . . . . . . . . 143

5.4 Selecting muon neutrino and anti-neutrino events . . . . . . . 144

5.5 Summary. . . . . . . . . . . . . . . . 150

$\begin{array}{lll}\text { Chapter 6. Muon neutrino events in the near detector } & 151\end{array}$

6.1 Calibration of the near detector magnetic field . . . . . . . . . 152

6.2 Selecting near detector events . . . . . . . . . . . . . . . . 161

6.3 Near detector data . . . . . . . . . . . . . . . . . 163

6.4 Summary. . . . . . . . . . . . . . . . . . 177

$\begin{array}{lll}\text { Chapter 7. } & \text { Tuning of flux and cross-section models } & 178\end{array}$

7.1 Parameterization of the flux model . . . . . . . . . . . . . . 179

7.2 Parameterization of the cross-section model . . . . . . . . . . . 185

7.3 Fit description and $\chi^{2}$ function . . . . . . . . . . . . . . . . 188

7.4 Kernel method and $\chi^{2}$ function . . . . . . . . . . . . . . . 190

7.5 Tuning results . . . . . . . . . . . . . . . . . . . 192 
7.6 Study of different fit approaches . . . . . . . . . . . . . 199

7.7 Summary . . . . . . . . . . . . . . . . . 202

Chapter 8. Muon neutrino events in the far detector 204

8.1 Extrapolation from near to far detectors . . . . . . . . . . 204

8.2 Selecting far detector events . . . . . . . . . . . . . . . 209

8.3 Far detector data . . . . . . . . . . . . . . . . . . . . 213

8.4 Summary . . . . . . . . . . . . . . . . . . . . . . 219

Chapter 9. Measurement of muon neutrino disappearance 220

9.1 Description of the fit method . . . . . . . . . . . . 220

9.2 Measurement of neutrino oscillations parameters . . . . . . . 223

9.3 Statistically independent pseudo-experiments . . . . . . . . 235

9.4 Treatment of the systematic errors . . . . . . . . . . . . . 243

9.5 MINOS results . . . . . . . . . . . . . . . . . . 251

9.6 Conclusions . . . . . . . . . . . . . . . . 256

$\begin{array}{ll}\text { Appendices } & 257\end{array}$

Appendix A. Summary of kinematic distributions 258

$\begin{array}{ll}\text { Bibliography } & 267\end{array}$

$\begin{array}{ll}\text { Vita } & 280\end{array}$ 


\section{Chapter 1}

\section{Introduction to neutrino physics}

Particle physics is the study of the elementary constituents (or particles) of the physical world. We have learned a great deal about elementary particles since 1896, when Henry Becquerel discovered radioactivity. Subsequent work by Marie and Pierre Curie led to a realization that this phenomena is linked to the interior structure of atoms. Much of modern physics, including neutrino physics, is rooted in those discoveries. This chapter presents a necessarily brief history of neutrinos, from their discovery to modern neutrino experiments.

\subsection{A brief history of neutrinos}

In 1914, James Chadwick showed that a spectrum of electrons emitted in the $\beta$-decays is continuous [1]:

$$
N \rightarrow X+e^{-},
$$

where $\mathrm{N}$ and $\mathrm{X}$ stand for the initial and final nucleus states. It was observed, however, that the continuous electron spectrum violated the energy conservation principle if the $\beta$-decays emit a single particle. At that time, this observation was explained by interactions of the electron with nearby atoms, 
before the electron was detected. In 1927, Charles Ellis and William Wooster performed calorimetric measurements that unequivocally proved that in the nuclear $\beta$-decays electrons are emitted over a broad range of energies [2].

A solution to this puzzle was proposed in 1930 by Wolfgang Pauli, who introduced a new neutral particle. Experimental data suggested that this particle is significantly lighter than a proton and very penetrating to escape observations; it also needed to have spin- $\frac{1}{2}$ to explain statistics of the $\beta$-decays. Pauli wrote: "I have hit upon a desperate remedy to save the 'exchange theorem' of statistics and the law of conservation of energy. Namely, the possibility that in the nuclei there could exist electrically neutral particles, which I will call neutrons... The continuous beta spectrum would then make sense with the assumption that in beta decay, in addition to the electron, a neutron is emitted such that the sum of the energies of neutron and electron is constant." 1 In 1932, Chadwick discovered a neutron [3], but it was too heavy to be the particle predicted by Pauli. Enrico Fermi then developed a theory of the $\beta$-decays [4]; this theory accurately described the existing data and included the new neutral particle - which Fermi renamed as neutrino.

As Pauli suggested, the neutrino is emitted together with the electron in the nuclear $\beta$-decays in the reaction:

$$
{ }^{A} Z \rightarrow{ }^{A}(Z+1)+e^{-}+\nu
$$

where ${ }^{A} Z$ stands for the nucleus with $Z$ protons and $A-Z$ neutrons and the

\footnotetext{
${ }^{1}$ Translation by Kurt Riesselmann.
} 
neutrino ${ }^{2}$ escapes unobserved. The $\beta$-decays are now used to set a limit on the mass of the emitted neutrino by accurately measuring electron momentum and nuclear recoil energy. The current measurements constrain the mass of the neutrino produced in the tritium $\beta$-decays to be less than $2 \mathrm{eV}$ [5]. In comparison, the electron mass is $510,998 \mathrm{eV}$, and the proton mass is $938,272,029 \mathrm{eV}[5] .^{3}$

Neutrinos were directly observed for the first time in 1956 by Frederick Reines and Clyde Cowan [6]. Their experiment detected electron antineutrinos produced by the Savannah River nuclear plant in South Carolina, using a detector placed behind heavy shielding material. Electron anti-neutrinos interact with protons via the reaction $\bar{\nu}+p \rightarrow n+e^{+}$. The detector observed this reaction via a coincidental measurement of a neutron capture on cadmium and positron annihilation. The new coincidence method substantially reduced a number of background events in the detector. Other physicists, including Raymond Davis Jr., had unsuccessfully tried to detect neutrinos at the same site via the reaction $\nu+{ }^{37} \mathrm{Cl} \rightarrow e^{-}+{ }^{37} \mathrm{Ar}$. A use of this reaction for the detection of neutrinos was suggested by Bruno Pontecorvo and Luis Alvarez $[7,8]$. These attempts were unsuccessful because this reaction is only sensitive to neutrinos, rather than anti-neutrinos. In the 1960s, Davis used this reaction for the first time to detect neutrinos produced in the sun [9]. Davis measured fewer than expected solar neutrinos giving rise to "the solar neutrino problem."

\footnotetext{
${ }^{2}$ This particle is now known as an electron anti-neutrino.

${ }^{3}$ Here and throughout the thesis, the convention $c^{2}=1$ is used, where $c$ is the speed of light.
} 
In the two decades preceding the detection of neutrinos, muons and pions were discovered in cosmic ray experiments. The pions were observed to decay to muons through a process similar to the $\beta$-decay: $\pi \rightarrow \mu+\nu$. In 1962, an experiment led by Melvin Schwartz, Leon Lederman, and Jack Steinberger concluded that the interaction of the high energy neutrinos with matter produces muons [10]. Because these neutrinos were produced in the decay of pions, the researchers concluded that the neutrinos associated with the pion decays are different from the neutrinos produced in the $\beta$-decays. This new type of neutrinos was named muon neutrinos. A coherent picture of two "generations" of particles emerged where electron and its neutrino are the first generation, and muon and its neutrino are the second generation. This family of particles was called leptons.

With the help of new particle accelerators, many new particles were discovered during the 1960s. These particles shared common properties with pions and protons. In 1964, Murray Gell-Mann and George Zweig proposed the idea that this family of particles ${ }^{4}$, which included pions and protons but not leptons, is composed of even more elementary constituents called quarks. It was observed in a proton-electron scattering experiment at SLAC that protons contain point-like constituents. This and other experiments provided data for the development of the "Standard Model of Particles and Fields." This Standard Model included the Glashow-Weinberg-Salam theory of electroweak interactions. This theory predicted the existence of three heavy gauge bosons,

\footnotetext{
${ }^{4}$ These particles are now collectively known as hadrons
} 


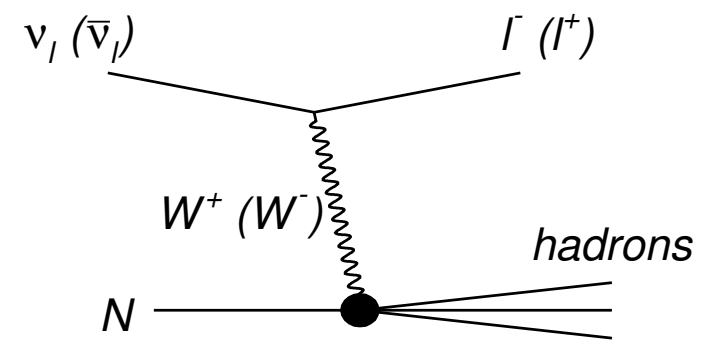

(a) Charged-current

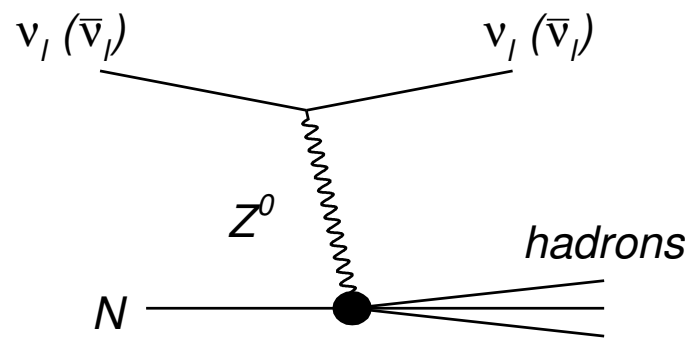

(b) Neutral-current

Figure 1.1: Feynman diagrams for charged-current and neutral current interactions of neutrinos. Neutrinos interact with quarks bound within protons and neutrons which form nuclei. Neutrinos can be distinguished by flavor of associated charged lepton (electron, muon or tau) in the charged current interactions.

$W^{ \pm}$and $Z^{0}$, and a fourth (charm) quark. In 1974, the discovery of the $J / \Psi$ particle $[11,12]$ confirmed the existence of the charm quark and established a second generation of quarks.

The theory predicted the existence of the heavy gauge bosons, $W^{ \pm}$ and $Z^{0}$, which mediate charged-current $(\mathrm{CC})$ and neutral-current $(\mathrm{NC})$ interactions. Figure 1.1 illustrates the neutral-current and charged-current interactions of neutrinos with quark constituents of protons and neutrons bound within a nucleus. In the charged-current interactions, a charged lepton is produced along with an excited hadronic system. In the neutral-current interactions, a neutrino is produced along with an excited hadronic system. Neutrinos can also scatter off atomic electrons through the quasielastic scattering process $\nu+e^{-} \rightarrow \nu+e^{-}$. In 1973, the neutral-current interactions were detected at CERN [13], confirming the existence of the neutral gauge bozon $Z^{0}$. 
The 1970s saw rapid progress in experimental and theoretical particle physics. The high energy neutrinos were used as probes of a nucleon structure in deep-inelastic scattering experiments. These experiments helped to firmly establish the quark theory. The discovery of the b-quark [14] and the tau lepton [15] established the existence of three generations of leptons and quarks.

The Standard Model combines both electroweak and strong fields and three generations of the leptons and quarks (summarized in Table 1.1). With a new high energy accelerator and large detectors, the heavy gauge bosons, $Z^{0}$ and $W^{ \pm}$, were directly observed in 1983 at CERN $[16,17]$. In the late 1980s, experiments at CERN and SLAC studied the decay of the $Z$ boson and determined the number of light neutrinos ${ }^{5}, N_{\nu}=2.984 \pm 0.008$ [5]. A sixth (top) quark was detected in 1995 [18], and a third (tau) neutrino was observed in 2000 by the DONUT experiment [19]. This experiment observed the decays of the tau leptons produced in the $\nu_{\tau}$ charged-current interactions. The Standard Model also includes a Higgs boson, which is the last undetected particle predicted by the model. The search for the Higgs boson is the goal of the newly constructed Large Hadron Collider at CERN.

\subsection{Theory of neutrino oscillations}

The Standard Model describes all existing data on the weak, electromagnetic and strong interactions [5]. The weak interactions are due to cou-

\footnotetext{
${ }^{5}$ The light neutrinos have mass less than one half of the mass of the $Z$ boson, $91.187 \mathrm{GeV}[5]$.
} 


\begin{tabular}{l|r|r} 
Quark flavor & Mass $(\mathrm{MeV})$ & Electric charge \\
\hline \hline Up & 2.5 & $2 / 3$ \\
Down & 5.0 & $-1 / 3$ \\
\hline \hline Charm & 1,270 & $2 / 3$ \\
Strange & 104 & $-1 / 3$ \\
\hline \hline Top & 171,000 & $2 / 3$ \\
Bottom & 4,200 & $-1 / 3$
\end{tabular}

\begin{tabular}{l|r|r} 
Lepton flavor & Mass $(\mathrm{MeV})$ & Electric charge \\
\hline \hline Electron neutrino $\left(\nu_{\mathbf{e}}\right)$ & $<2 \times 10^{-6}$ & 0 \\
Electron $(\mathrm{e})$ & 0.51 & -1 \\
\hline \hline Muon neutrino $\left(\nu_{\mu}\right)$ & $<0.19$ & 0 \\
Muon $(\mu)$ & 105.7 & -1 \\
\hline \hline Tau neutrino $\left(\nu_{\tau}\right)$ & $<18.2$ & 0 \\
Tau $(\tau)$ & 1776.8 & -1
\end{tabular}

Table 1.1: Three generations (or flavors) of fermions in the Standard Model. The table shows the most current mass measurements and limits [5]. The quark mass measurements contain significant uncertainties because quarks are confined within hadrons, and, unlike leptons, their masses can not be measured directly. The neutrino mass limits are obtained from direct measurements. The squared neutrino mass differences are measured precisely by neutrino oscillation experiments. 
plings of the leptons and quarks with the three heavy vector gauge bosons, $W^{ \pm}$ and $Z^{0}$. There are three flavors of leptons and quarks, summarized in Table 1.1. Each flavor of lepton and quark has a different mass, but all other characteristics are the same. The weak interactions couple to linear superpositions of the quark mass states, as described by the Cabbibo-Kobayashi-Maskawa (CKM) matrix (see Reference [5] for a review), this results in a flavor violation in the quark sector.

Neutrino mixing is a process in which a neutrino of one flavor is converted into a different flavor. This process does not conserve a number of leptons with one flavor. There are no experimental measurements to suggest a lepton number violation for the charged leptons [5]. For example, the branching ratio for the lepton number violating process $\mu^{-} \rightarrow e^{-}+\gamma$ is less than $1.2 \times 10^{-11}$. The neutrino mixing allows the lepton number violation only if neutrinos have non-zero masses. In 1957, Bruno Pontecorvo [20,21] proposed that a neutrino state produced in the weak interactions is a superposition of two neutrino mass states resulting in the neutrino mixing. This process is similar to the mixing of neutral kaon states. The possibility of the muon and electron neutrino mixing was first considered by Ziro Maki, Masami Nakagawa, and Shoichi Sakata [22].

The weak vector gauge bosons couple to left-handed neutrino flavor states $\nu_{\alpha}$, identified by the charged lepton flavor $\alpha=\mathrm{e}, \mu, \tau$. It is assumed that the neutrinos are Dirac fields; the current experimental data do not distinguish 
between Majoranna and Dirac neutrino masses. ${ }^{6}$ Neutrino mass states $\nu_{i}$. are expressed as linear superpositions of the flavor states:

$$
\left|\nu_{i}\right\rangle=\sum_{\alpha=\mathrm{e}, \mu, \tau} U_{\alpha i}\left|\nu_{\alpha}\right\rangle
$$

where $U$ is the $3 \times 3$ unitary mixing matrix often referred to as the PontecorvoMaki-Nakagawa-Sakata (PMNS) matrix. This matrix can be inverted to express the neutrino flavor states as the linear superpositions of the mass states:

$$
\left|\nu_{\alpha}\right\rangle=\sum_{i=1,2,3} U_{\alpha i}^{*}\left|\nu_{i}\right\rangle
$$

The data do not exclude additional neutrino mass states [5]. However, no compelling evidence exists to suggest the existence of more than three neutrino mass states. For simplicity, this discussion considers the three neutrino mass states.

A neutrino is born in the definite flavor state $\nu_{\alpha}$. It propagates through empty space to a detector as a superposition of the mass states:

$$
\left|\nu_{\alpha}(\mathbf{x})\right\rangle=\sum_{i=1,2,3} U_{\alpha i}^{*} e^{-i \mathbf{x} \cdot \mathbf{p}_{i}}\left|\nu_{i}\right\rangle
$$

where $\mathbf{x}$ is the space-time four-vector, $\mathbf{p}_{i}$ is the energy-momentum four-vector, and the mass states propagate as quantum mechanical plane waves. A rigorous treatment using quantum mechanical wave packets gives identical results [24].

\footnotetext{
${ }^{6}$ See Reference [23] for a comprehensive review of different scenarios. This section follows the standard approach [5] to a derivation of the neutrino mixing parameters.
} 
Using Equation 1.1, the state $\left|\nu_{\alpha}(\mathbf{x})\right\rangle$ is expressed as the superposition of the flavor states:

$$
\left|\nu_{\alpha}(\mathbf{x})\right\rangle=\sum_{i=1,2,3} \sum_{\gamma=\mathrm{e}, \mu, \tau} U_{\alpha i}^{*} U_{\gamma i} e^{-i \mathbf{x} \cdot \mathbf{p}_{i}}\left|\nu_{\gamma}\right\rangle
$$

A transition probability to observe a flavor state $\nu_{\beta}$ at the detector position $\mathbf{x}$ is given by:

$$
\begin{aligned}
P\left(\nu_{\alpha} \rightarrow \nu_{\beta}\right) & =\left|\left\langle\nu_{\beta} \mid \nu_{\alpha}(\mathbf{x})\right\rangle\right|^{2}, \\
& =\sum_{i=1,2,3} \sum_{j=1,2,3} U_{\alpha i}^{*} U_{\beta i} U_{\alpha j} U_{\beta j}^{*} e^{i \mathbf{x} \cdot\left(\mathbf{p}_{i}-\mathbf{p}_{j}\right)} .
\end{aligned}
$$

The phase difference $\phi_{i j}$ is given by:

$$
\phi_{i j}=\mathbf{x} \cdot\left(\mathbf{p}_{i}-\mathbf{p}_{j}\right)=\left(p_{i}-p_{j}\right) L-\left(E_{i}-E_{j}\right) t
$$

where $L$ is the distance to a detector. The transit time, $t$, can be approximated as $L / \bar{v}$, where the average neutrino speed $\bar{v}$ :

$$
\bar{v}=\frac{p_{i}+p_{j}}{E_{i}+E_{j}}
$$

The phase differences are then approximated as:

$$
\phi_{i j} \approx \frac{p_{i}^{2}-p_{j}^{2}}{p_{i}+p_{j}} L-\frac{E_{i}^{2}-E_{j}^{2}}{p_{i}+p_{j}} L=\frac{m_{j}^{2}-m_{i}^{2}}{p_{i}+p_{j}} L \approx \frac{m_{j}^{2}-m_{i}^{2}}{2 E} L,
$$

where the neutrino momentum is approximately equal to the neutrino energy.

After performing few manipulations and using properties of the unitary matrix $U$, the transition probability is written as:

$$
\begin{aligned}
P\left(\nu_{\alpha} \rightarrow \nu_{\beta}\right) & =\delta_{\alpha \beta} \\
& -4 \sum_{i<j} \operatorname{Re}\left(U_{\alpha i}^{*} U_{\beta i} U_{\alpha j} U_{\beta j}^{*}\right) \sin ^{2}\left(\Delta m_{i j}^{2} L / 4 E\right) \\
& +2 \sum_{i<j} \operatorname{Im}\left(U_{\alpha i}^{*} U_{\beta i} U_{\alpha j} U_{\beta j}^{*}\right) \sin \left(\Delta m_{i j}^{2} L / 2 E\right),
\end{aligned}
$$


where Re and Im are the real and imaginary parts of a complex number. Many experiments, including MINOS, measure neutrino survival probability for the transition $\nu_{\alpha} \rightarrow \nu_{\alpha}$ :

$$
P\left(\nu_{\alpha} \rightarrow \nu_{\alpha}\right)=1-4 \sum_{i<j}\left|U_{\alpha i}^{*}\right|^{2}\left|U_{\alpha j}\right|^{2} \sin ^{2}\left(\Delta m_{i j} L / 4 E\right)
$$

A convenient parametrization of the mixing matrix $U$ was introduced in Reference [25]:

$$
U=\left(\begin{array}{ccc}
1 & 0 & 0 \\
0 & c_{23} & s_{23} \\
0 & -s_{23} & c_{23}
\end{array}\right) \times\left(\begin{array}{ccc}
c_{13} & 0 & s_{13} e^{-i \delta} \\
0 & 1 & 0 \\
s_{13} e^{-i \delta} & 0 & c_{13}
\end{array}\right) \times\left(\begin{array}{ccc}
c_{12} & s_{12} & 0 \\
-s_{12} & c_{12} & 0 \\
0 & 0 & 1
\end{array}\right)
$$

where $c_{i j}=\cos \theta_{i j}, s_{i j}=\sin \theta_{i j}$, and $\theta_{i j}$ is the mixing angle for the $\nu_{i}$ and $\nu_{j}$ flavor states.

The transition (or oscillation) probability, $P\left(\nu_{\alpha} \rightarrow \nu_{\alpha}\right)$, depends on the neutrino energy, the distance, and the mass squared differences as a function of $\sin \left(\Delta m_{i j}^{2} L / 4 E\right)$. The term "oscillations" is used because of the sinusoidal dependence on $L / E$. A size of the $\Delta m_{i j}^{2}$ splitting determines a baseline and energy range for an experiment searching for the neutrino oscillations. Experimental data suggest two very different scales for the mass differences squared, $\Delta m_{32}^{2} \gg \Delta m_{12}^{2}$. Experimental evidence also favors small values of the mixing angle $\theta_{13}$, compared to the mixing angles $\theta_{12}$ and $\theta_{23}, \theta_{13} \ll \theta_{12}$, and $\theta_{13} \ll \theta_{32}$. In this scenario, the three flavor neutrino oscillations described by Equation 1.9 can be reduced to a simple two-neutrino (or two-flavor) case. The mass squared difference between these two mass states is $\Delta m^{2}$. The $2 \times 2$ 
mixing matrix contains one mixing angle, omitting irrelevant phase factors. Equation 1.9 now reduces to:

$$
P\left(\nu_{\alpha} \rightarrow \nu_{\beta}\right)=\sin ^{2} 2 \theta \sin ^{2}\left(1.27 \Delta m^{2} L / E\right),
$$

when $\alpha \neq \beta$. The transition (or survival) probability of the same flavor neutrino is:

$$
P\left(\nu_{\alpha} \rightarrow \nu_{\alpha}\right)=1-\sin ^{2} 2 \theta \sin ^{2}\left(1.27 \Delta m^{2} L / E\right) .
$$

These two formulas accurately describe data for several neutrino oscillation experiments. One exception is solar neutrino experiments where matter effects need to be included. In Chapter 9, Equation 1.13 will be used for an analysis of $\nu_{\mu}$ events at the MINOS far detector. Figure 1.2 shows an expected probability to observe $\nu_{\mu}$ events at the MINOS far detector with the neutrino oscillations present.

\subsection{Experimental evidence for neutrino oscillations}

Neutrino oscillations are investigated using an intense source of neutrinos and a large neutrino detector. Neutrino oscillation experiments compare a number of neutrino interactions in the detector with an expected interaction rate, assuming that neutrinos travel directly from the source to the detector without oscillations. A detailed understanding of the neutrino source is required to compute the expected interaction rate. For neutrinos produced by the sun or cosmic rays, computation of the predicted rate presents a complex experimental and theoretical problem. Man-made sources of neutrinos are 


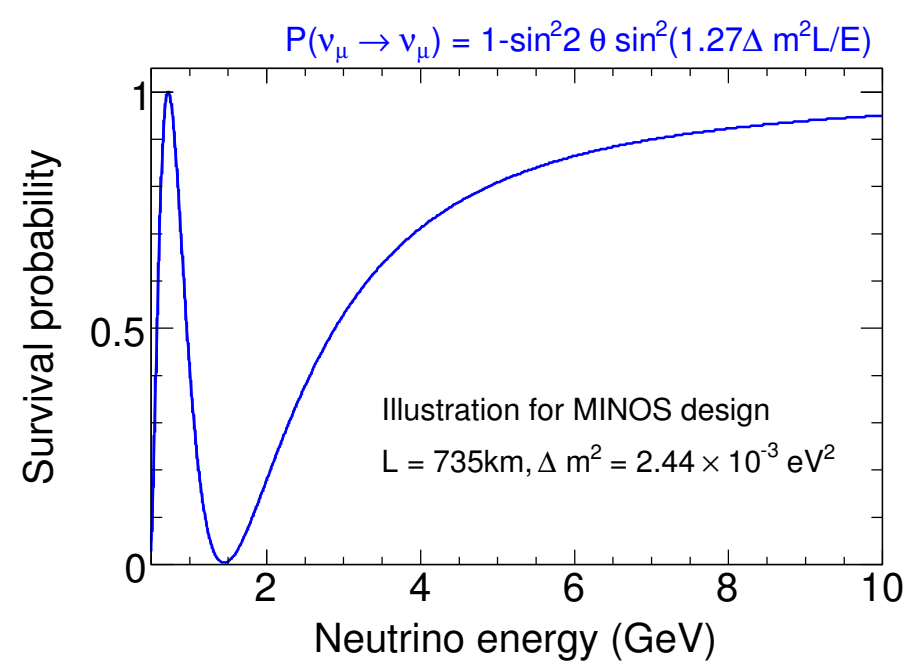

Figure 1.2: Muon neutrino survival probability plotted as a function of neutrino energy for the MINOS $735 \mathrm{~km}$ baseline and the mass squared difference measured in Chapter 9. The blue line shows the probability that a muon neutrino produced at Fermilab reaches the MINOS far detector.

easier to understand, but they generate less intense neutrino fluxes. The evidence for neutrino oscillations from experiments using natural and man-made neutrino sources is discussed in this section.

\subsubsection{Electron neutrinos from the sun}

A first hint of neutrino oscillations came from the radiochemical experiment at Homestake Gold Mine, South Dakota, led by Davis. The experiment was sensitive to the electron neutrinos produced by the sun via the reaction $\nu+{ }^{37} \mathrm{Cl} \rightarrow e^{-}+{ }^{37}$ Ar with the threshold energy $0.81 \mathrm{MeV}$. Argon isotopes decay via electron capture with a half-life of 35 days. Davis successfully solved the experimental challenge of being able to extract around 17 expected argon 


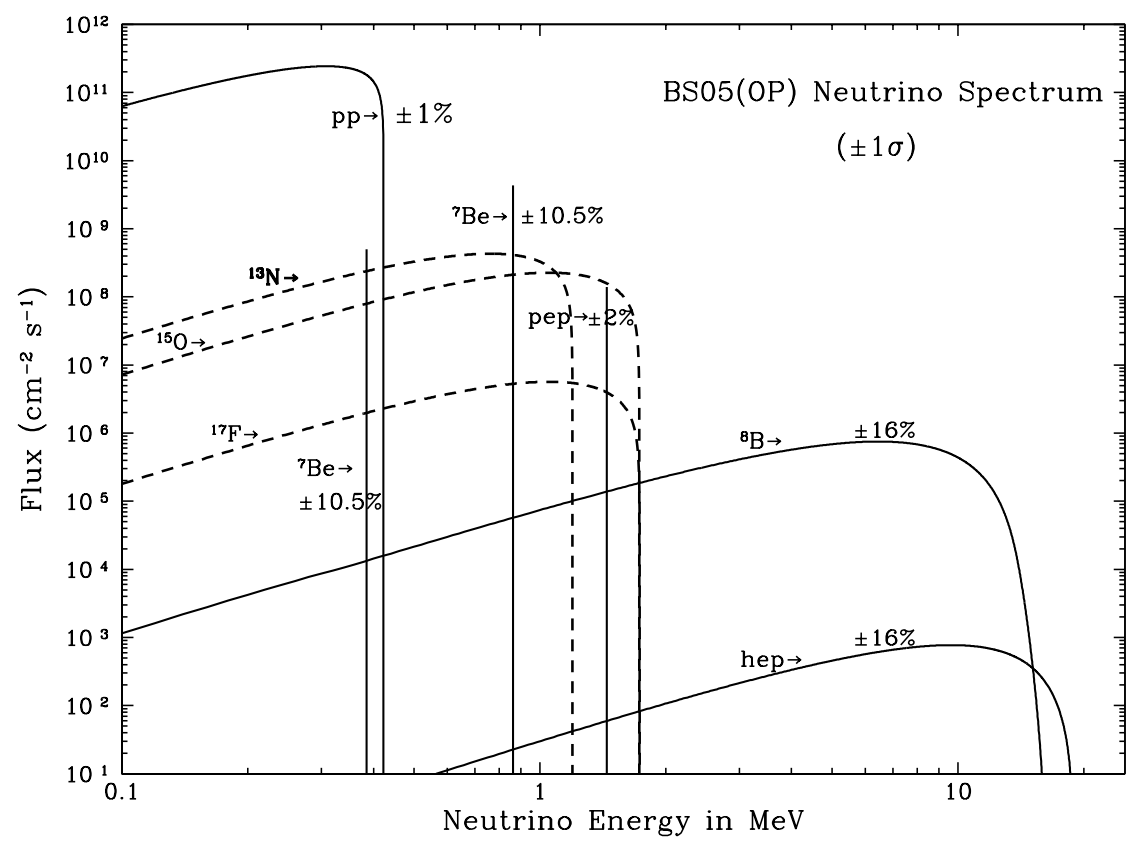

Figure 1.3: Predicted solar neutrino energy spectrum [27].

atoms from a sea of $2 \times 10^{30}$ chlorine atoms every two months [26]. The experiment started collecting data in the late 1960s and completed its run in 1994. Through this period the experiment measured a consistent rate of argon capture, and the final result for the production of argon atoms per day was $2.56 \pm 0.16$ (stat) \pm 0.16 (syst) SNU $(1 \mathrm{SNU}=1$ Solar Neutrino Unit $=1$ capture per second and per 1,036 target atoms).

Through the efforts of many scientists, a cycle for the energy production in the sun was understood by 1960s. A crucial comparison for the Davis experiment was a new computation for the number of neutrinos produced in the sun with energies greater than the reaction threshold $0.81 \mathrm{MeV}$. The predicted solar neutrino spectrum for the current solar model is shown in 
Figure 1.3. The sun generates energy via the nuclear conversion reaction, which schematically is expressed as:

$$
4 p \rightarrow^{4} H e+2 e^{+}+2 \nu_{e}
$$

Two neutrino are produced in each fusion reaction. These neutrinos escape the sun and carry away some energy. John Bahcall computed the expected number of argon atoms produced in the Davis experiment as $40 \pm 20 \mathrm{SNU}$ [28]. This discrepancy between the measured and expected rates for the number of the captured argon atoms posed "the solar neutrino problem."

New experiments were constructed to study the electron neutrinos produced by the sun. All of these experiments confirmed that the number of observed solar electron neutrino events were less than the number of these events predicted by a theoretical model of the sun. Three new experiments used a radiochemical method based on gallium. ${ }^{7}$ This method is sensitive to the low energy solar neutrinos through the reaction ${ }^{71} G a+\nu_{e} \rightarrow{ }^{71} G e+e^{-}$with an energy threshold of $0.233 \mathrm{MeV}$. The measured capture rate of ${ }^{71} \mathrm{Ge}$ isotopes in these three experiments was significantly smaller than the predicted rate.

In Japan, Kamiokande and Super-Kamiokande detectors were built to study the proton decay $p \rightarrow \pi^{0}+e^{+}$. These experiments detect charged particles via Cherenkov radiation in a large tank of pure water. The Kamiokande detector was built in the late 1980s. It was later replaced by the SuperKamiokande detector, which holds 50,000 tons of water. Interactions of solar

\footnotetext{
${ }^{7}$ See Reference [5] for a review.
} 
neutrinos are detected in real-time using the elastic scattering (ES) reaction:

$$
\begin{aligned}
& \nu_{x}+e^{-} \rightarrow \nu_{x}+e^{-} \text {(via neutral-current) } \\
& \nu_{e}+e^{-} \rightarrow \nu_{e}+e^{-} \text {(via charged-current) }
\end{aligned}
$$

where $x=e, \mu, \tau$. The neutral-current reaction measures interaction rates of neutrinos of all flavors. However, the sensitivity of the ES reaction to $\nu_{\mu}$ and $\nu_{\tau}$ is smaller than the sensitivity to $\nu_{\mathrm{e}}, \sigma\left(\nu_{\mu, \tau}\right) \approx 0.16 \sigma\left(\nu_{e}\right)$, because the sensitivity to $\nu_{\mathrm{e}}$ is enhanced through the charged-current interactions. In the ES process, a direction of the outgoing electron is measured by the detector. This signature helps to cleanly separate solar neutrino events from background events. For events over the $5 \mathrm{MeV}$ threshold in Super-Kamiokande, the measured flux of the solar neutrinos was $(2.35 \pm 0.02$ (stat.) \pm 0.08 (syst.) $) \times 10^{6} \mathrm{~cm}^{-2} \mathrm{sec}^{-1}[29]$. This measured flux is significantly smaller than the predicted flux $(5.69 \pm 1.0) \times$ $10^{6} \mathrm{~cm}^{-2} \mathrm{sec}^{-1}[27]$. This result agreed with other solar neutrino experiments.

The Sudbury Neutrino Observatory (SNO) was a solar neutrino experiment in the Creighton mine near Sudbury, Ontario, Canada. This experiment started observations in 1999 and completed in 2006. SNO was a water-Cherenkov detector that used ultra-pure, heavy water $\left(D_{2} O\right)$ as a neutrino target. The low binding energy of a proton and neutron in a deuterium atom $^{8}$ gave SNO a unique sensitivity to the low energy neutral-current and charged-current interactions. The experiment was sensitive to the following

\footnotetext{
${ }^{8}$ For oxygen, the nucleon binding energy of few $\mathrm{MeV}$ suppresses these reactions
} 
three reactions:

$$
\begin{array}{cl}
\nu_{x}+e^{-} \rightarrow \nu_{x}+e_{-} & \text {(elastic scattering) }, \\
\nu_{e}+d \rightarrow e^{-}+p+p & \left(\nu_{\mathrm{e}} \text { charged-current }\right), \\
\nu_{x}+d \rightarrow \nu_{x}+p+n & \text { (neutral-current) }
\end{array}
$$

where $x=e, \mu, \tau$. The elastic scattering and neutral-current interactions are sensitive to all neutrino flavors. They provide a direct measurement of the total solar neutrino flux. The $\nu_{\mathrm{e}}$ charged-current interactions are only sensitive to electron neutrinos. The previous solar experiments were mostly sensitive to the flux of electron neutrinos. SNO independently measured the electron neutrino flux and total neutrino flux from the sun. In the last phase of the SNO experiment, the following neutrino fluxes were measured (in units of $\left.10^{6} \mathrm{~cm}^{-2} \mathrm{~s}^{-1}\right)[30]:$

$$
\begin{aligned}
\phi_{\mathrm{CC}}^{\mathrm{SNO}} & =1.67_{-0.04}^{+0.05}(\text { stat })_{-0.08}^{+0.07}(\text { syst }) \\
\phi_{\mathrm{ES}}^{\mathrm{SNO}} & =1.77_{-0.21}^{+0.24}(\text { stat })_{-0.10}^{+0.09}(\text { syst }) \\
\phi_{\mathrm{NC}}^{\mathrm{SNO}} & =5.54_{-0.31}^{+0.33}(\text { stat })_{-0.34}^{+0.36} \text { (syst) },
\end{aligned}
$$

and the ratio of the solar neutrino flux measured with the $\nu_{\mathrm{e}}$ charged-current and neutral-current reactions was:

$$
\frac{\phi_{\mathrm{CC}}^{\mathrm{SNO}}}{\phi_{\mathrm{NC}}^{\mathrm{SNO}}}=0.301 \pm 0.033(\text { total })
$$

This measurement indicates that the muon and tau neutrinos are present among the solar neutrinos. These neutrino flavors are not generated by the 


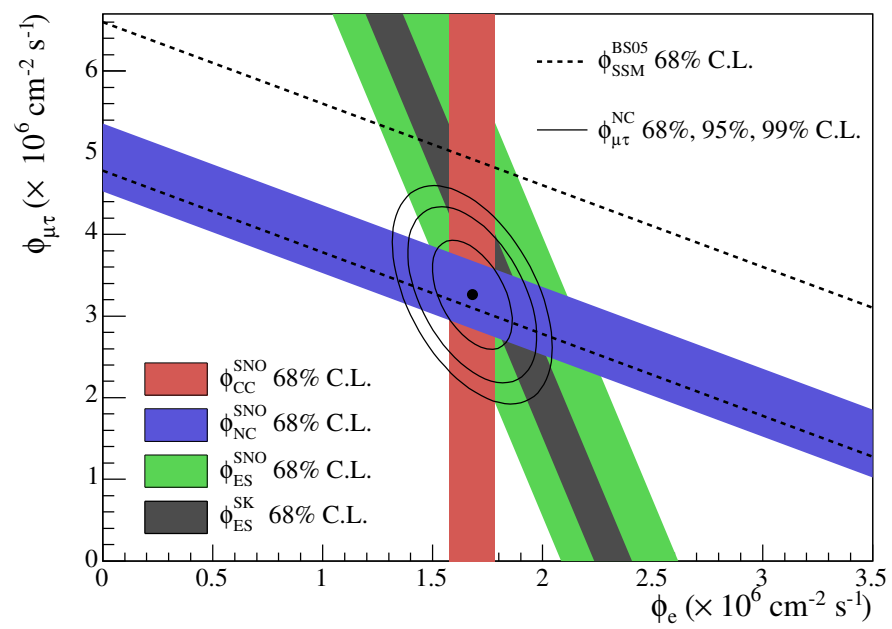

Figure 1.4: Flux of $\mu+\tau$ neutrinos versus flux of electron neutrinos. CC, NC and ES flux measurements are indicated by the filled bands. The predicted ${ }^{8} \mathrm{~B}$ solar neutrino flux [27] is shown as dashed lines, and that measured with the $\mathrm{NC}$ channel is shown as the solid band parallel to the prediction. The narrow band parallel to the SNO ES result corresponds to the Super-Kamiokande result [31]. The intercepts of these bands with the axes represent the $\pm 1 \sigma$ uncertainties. The non-zero value of $\phi_{\mu \tau}$ provides strong evidence for neutrino flavor transformation. The point represents $\phi_{e}$ from the CC flux and $\phi_{\mu \tau}$ from the NC-CC difference with $68 \%, 95 \%$, and $99 \%$ C.L. contours included. The figure and caption were taken from [32].

nuclear energy cycle in the sun, so a new explanation is required. The combination of the solar neutrino measurements from SNO and Super-Kamiokande is shown in Figure 1.4. This figure shows the earlier SNO result than the result discussed above. The two experiments consistently measured fewer than expected solar electron neutrinos.

The solution of the solar neutrino problem was proposed by Bruno Pontecorvo and Vladimir Gribov, who suggested that electron neutrinos con- 
vert to muon and tau neutrinos via neutrino oscillations [33,34]. The original theory was modified by Lincoln Wolfenstein, Stanislav Mikheyev, and Alexei

Smirnov $[35,35]$ to account for resonant effects introduced by the interaction of neutrinos with the matter inside the sun. The deficit of the electron neutrinos from the sun is now explained by oscillations of the electron neutrinos into the tau or muon neutrinos.

\subsubsection{Electron anti-neutrinos from nuclear reactors}

A remarkable confirmation of the solar neutrino oscillation hypothesis was carried out by the Kamioka Liquid-scintillator Anti-Neutrino Detector (KamLAND). This experiment observes interactions of electron anti-neutrinos through the inverse $\beta$-decay reaction:

$$
\bar{\nu}_{e}+p \rightarrow e^{+}+n
$$

The positron deposits energy in the scintillator and then annihilates. The measured positron energy deposition is directly related to the energy of the incoming neutrino. The neutron is captured on a proton after approximately $200 \mu \mathrm{s}$, generating a photon through the reaction:

$$
n+p \rightarrow d+\gamma(2.2 \mathrm{MeV})
$$

The delayed coincidence of the initial light from the positron and delayed light from the neutron capture is an extremely robust signature of electron anti-neutrinos. 


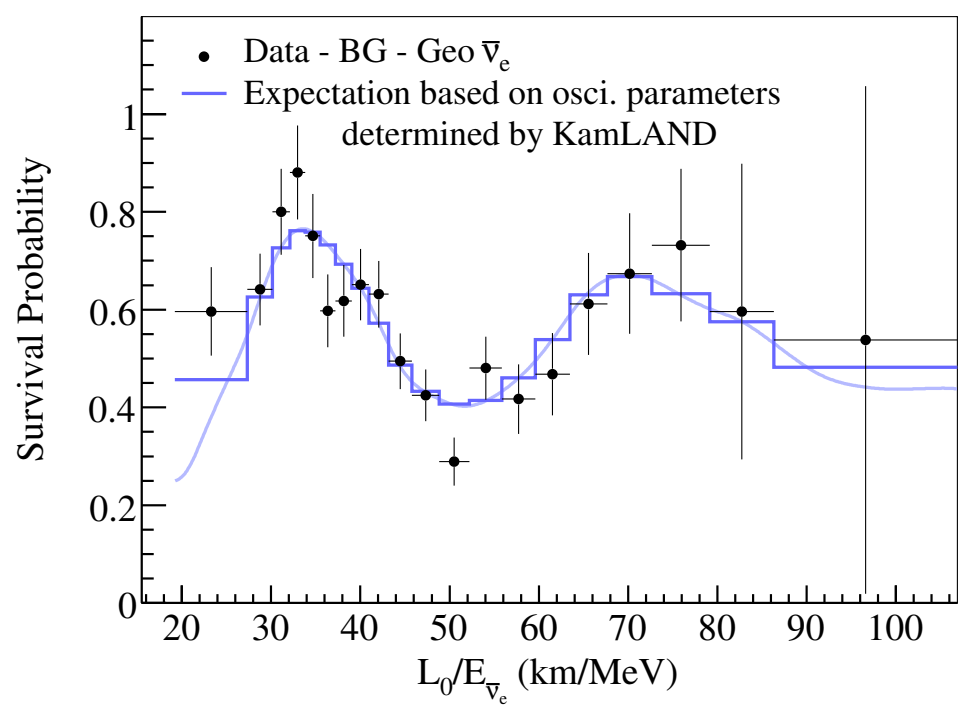

Figure 1.5: Ratio of the background subtracted electron anti-neutrino spectrum measured by KamLAND to the expectation for no-oscillation as a function of $\mathrm{L} / \mathrm{E}$. L is the effective baseline taken as a flux-weighted average $(\mathrm{L}=180 \mathrm{~km})$. The expected spectrum accounts for the distances to the individual reactors, time-dependent flux variations, and efficiencies. This figure was taken from [36].

Electron anti-neutrinos observed by KamLAND arrive from commercial nuclear reactors in Japan. The average distance between KamLAND and the reactors is $180 \mathrm{~km}$. The expected flux of anti-neutrinos at the detector can be predicted based on power production at the individual nuclear plants and the distance between each plant and the detector. Figure 1.5 shows a ratio of the observed and expected energy spectra for the electron anti-neutrino events. The observed data are accurately described by the two-neutrino oscillation formula, shown in Equation 1.13. The measured mass squared difference is $\Delta m^{2}=7.58_{-0.13}^{+0.14}(\text { stat })_{-0.15}^{+0.15}($ syst $) \times 10^{-5} \mathrm{eV}^{2}[36]$.

In addition, Bugey [37], Palo Verde [38], and CHOOZ [39] collabora- 
tions searched for neutrino oscillations using reactor nuclear plants as a source of electron anti-neutrinos. These smaller experiments were placed approximately $1 \mathrm{~km}$ away from a nuclear reactor, so they were sensitive to neutrino oscillations with the mass squared difference in the range $10^{-2}<\Delta m^{2}<$ $10^{-3} \mathrm{eV}^{2}$. A number of observed neutrino interactions in these experiments agreed with a prediction meaning that the electron anti-neutrinos do not oscillate in this mass parameter range.

\subsubsection{Atmospheric neutrinos}

In the 1990s, several deep underground neutrino experiments measured fluxes of atmospheric neutrinos. These neutrinos are produced by the interaction of cosmic rays (primarily protons) with the Earth's atmosphere, expressed schematically as:

$$
\begin{aligned}
p+N & \rightarrow N^{\prime}+\pi^{ \pm} / K^{ \pm} \\
\pi^{ \pm} / K^{ \pm} & \rightarrow \mu^{ \pm}+\nu_{\mu}\left(\bar{\nu}_{\mu}\right) \\
\mu^{ \pm} & \rightarrow e^{ \pm}+\bar{\nu}_{e}\left(\nu_{e}\right)+\nu_{\mu}\left(\bar{\nu}_{\mu}\right)
\end{aligned}
$$

This reaction produces approximately two muon neutrinos for every electron neutrino. The underground detectors measured the ratio of muon neutrinos to

electron neutrinos, $R=\frac{N_{\nu_{\mu}}+N_{\bar{\nu}_{\mu}}}{N_{\nu_{e}}+N_{\overline{\nu_{e}}}}$, for neutrino energies of few GeV. This ratio was expected to be approximately 2 , based on a detailed computation of the above reaction. The experiments consistently measured fewer muon neutrinos than were predicted, as illustrated in Figure 1.6. 


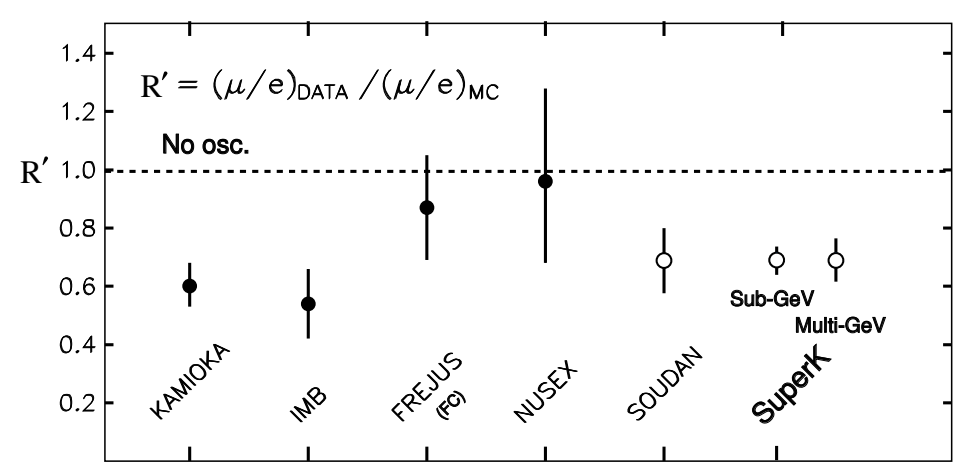

Figure 1.6: Measurements of the atmospheric neutrino flavor ratio of ratios $\mathrm{R}^{\prime}$ from several atmospheric neutrino experiments. See References $[40,41]$ for a review of the experiments. This figure was taken from [41].

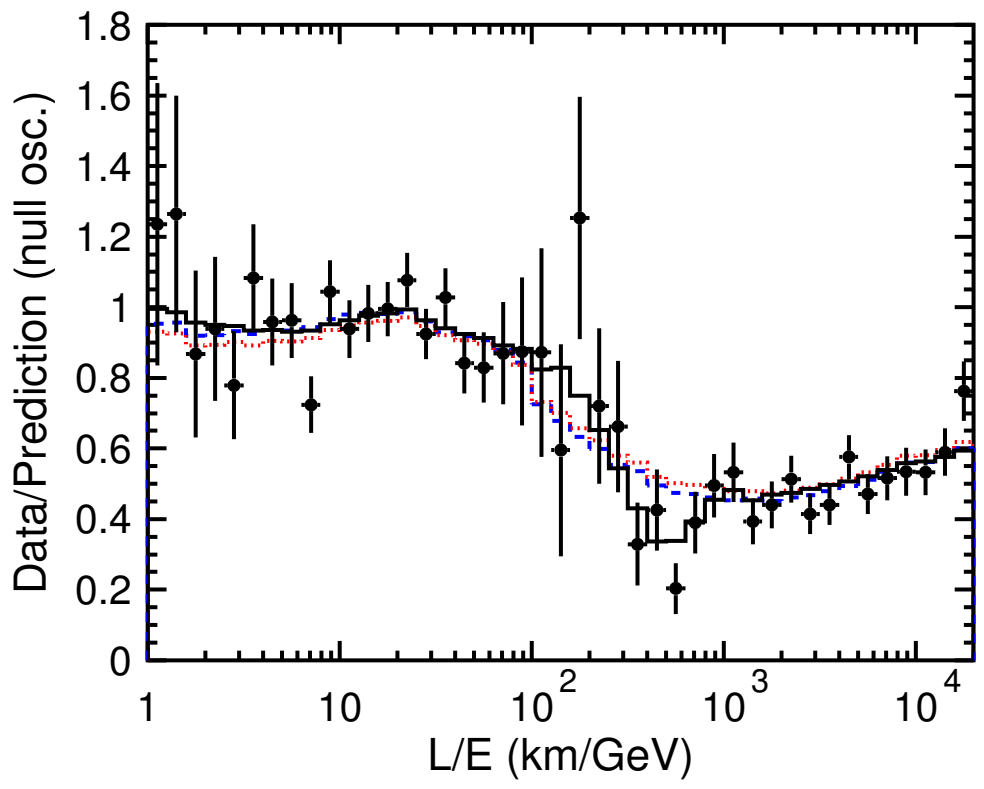

Figure 1.7: The figure shows a ratio of the Super-Kamiokande data to the expected events without neutrino oscillations (black points) plotted as a function of $\mathrm{L} / \mathrm{E}$. $\mathrm{L}$ is a reconstructed distance from the detector to the point in the Earth's atmosphere where the muon neutrino was produced. The solid black line shows the result of the two-neutrino $\nu_{\mu} \rightarrow \nu_{\tau}$ oscillations. The best-fit expectation for neutrino decay (dashed line) and neutrino decoherence (dotted line) are also shown. This figure was taken from [42]. 
The Super-Kamiokande experiment provided a definite measurement which proved that the atmospheric muon neutrino deficit (or disappearance) is due to the neutrino oscillations. The flux of cosmic rays is isotropic so the flux of atmospheric neutrinos is also isotropic. Super-Kamiokande measured variations in the muon neutrino flux as a function of the zenith angle $\theta$ [43]. These variations were consistent with the two-neutrino oscillation formula shown in Equation 1.13. The distance $\mathrm{L}$ is a reconstructed distance from the detector to the point in the Earth's atmosphere where the muon neutrino was produced. Figure 1.7 shows a ratio of the observed and expected muon neutrino fluxes as a function of $\mathrm{L} / \mathrm{E}$. The mass parameter that gives the best description of the Super-Kamiokande data is $\Delta m^{2}=2.4 \times 10^{-3} \mathrm{eV}^{2}[42]$. Figure 1.7 also shows the best-fit expectations for neutrino decay [44] and neutrino decoherence [45] models. These two models provide alternative explanations for the disappearance of atmospheric muon neutrinos. The recent MINOS result [46] disfavors these two models compared to the neutrino oscillation hypothesis.

\subsubsection{Man-made muon neutrino beams}

The first man-made, neutrino beam was constructed at Brookhaven, Upton, New York. In 1962, this beam was used to detect muon neutrinos. Neutrino beam facilities (beamlines) were later constructed at CERN, Fermilab, KEK, Los Alamos, and Serpukhov, where they were utilized to study weak interactions. These beamlines typically had a detector placed close to a source of neutrinos, producing a sufficiently high interaction rate in the detector with 
a mass of around one kton.

In the early 1990s, atmospheric neutrino experiments provided the first hints for the muon neutrino oscillations with $\mathrm{L} / \mathrm{E}$ around $400 \mathrm{~km} / \mathrm{GeV}$ $\left(\Delta m^{2} \approx 10^{-3} \mathrm{eV}^{2}\right)$. The muon neutrino oscillations with these parameters can be studied with an intense muon neutrino beam and a detector placed a few hundred kilometers away from a source of neutrinos.

The construction of these high intensity neutrino beams presents experimental challenges because a very high neutrino flux is required to produce a sufficient number of interactions in the detector a few hundred kilometers away. ${ }^{9}$ A chief advantage of the man-made neutrino beams is the significant reduction of systematic errors in the measurement of the neutrino oscillation parameters. An experiment utilizes two detectors. A smaller (near) detector is placed very close to the neutrino source, and a larger (far) detector is placed a few hundred km away from the neutrino source. These experiments use a differential measurement between two similar detectors to measure precisely the neutrino oscillation parameters.

Over the past few years, new muon neutrino beamlines were constructed at KEK, Fermilab, and CERN to study neutrino oscillations. The K2K experiment, which ran between 1999 and 2004, used a beamline at KEK aimed at the Super-Kamiokande detector with a baseline of $250 \mathrm{~km}$. The experiment observed a disappearance of muon neutrinos consistent with the Super-

\footnotetext{
${ }^{9}$ Reference [47] reviews recently constructed accelerator neutrino beams and discusses experimental innovations required to build these facilities.
} 
Kamiokande result for the atmospheric muon neutrino disappearance [48]. The MINOS experiment uses the NuMI beamline at Fermilab. A precise measurement of muon neutrino disappearance using the MINOS detectors and NuMI beamline is the subject of this thesis. The CNGS beamline at CERN is aimed at the OPERA detector. This experiment has recently started recording data [49]; its goal is to search for the appearance of tau neutrinos in the muon neutrino beam. The $\mathrm{T} 2 \mathrm{~K}$ experiment is expected to start operations next year [50]; it will search for the appearance of electron neutrinos in the muon neutrino beam.

\subsection{Summary of neutrino oscillations}

Experimental data provide compelling evidence that neutrinos do oscillate between three mass states. ${ }^{10}$ The values of the mass differences among these states are illustrated in Figure 1.8. Figure 1.9 shows the allowed region for the two-flavor oscillation parameters for the current solar and reactor experiments. Solar and reactor neutrino data are consistent with $\nu_{\mathrm{e}} \rightarrow \nu_{\mu}$ or $\nu_{\mathrm{e}} \rightarrow \nu_{\tau}$ oscillations with $\left|\Delta m^{2}\right|=7.58 \times 10^{-5} \mathrm{eV}^{2}$. However, these experiments do not tell us which of these two oscillation scenarios is realized in Nature.

The atmospheric, accelerator, and reactor neutrino data are consistent with pure $\nu_{\mu} \rightarrow \nu_{\tau}$ oscillations with $\left|\Delta m^{2}\right|=2.44 \times 10^{-3} \mathrm{eV}^{2}$. The MINOS experiment provides the most precise current measurement of $\left|\Delta m^{2}\right|[46]$. Fig-

\footnotetext{
${ }^{10}$ The LSND experiment suggests a fourth mass state but the MiniBooNE experiment did not confirm this hypothesis; see Reference [5] for a recent review.
} 


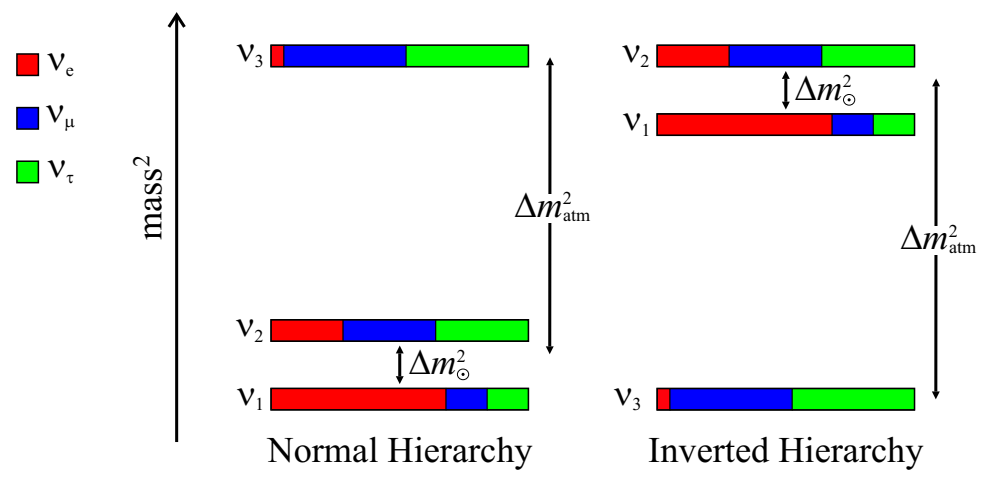

Figure 1.8: A three-neutrino squared-mass spectrum that accounts for the observed flavor changes of solar, reactor, atmospheric, and long-baseline accelerator neutrinos. The figures shows the normal and inverted mass hierarchies. The $\nu_{e}$ fraction in the $\nu_{3}$ mass eigenstate shows the best experimental limit. The plot was taken from [51].

ure 1.9 shows the allowed region for the two-flavor oscillation parameters for the muon neutrino disappearance. Super-Kamiokande data and null results from the short baseline reactor experiments exclude $\nu_{\mu} \rightarrow \nu_{\mathrm{e}}$ oscillations for this value of $\Delta m^{2}$, except for a very small value of the mixing angle $\theta_{13}$.

\subsection{Outline of the dissertation}

This dissertation presents a measurement of muon neutrino disappearance using the MINOS detectors and NuMI neutrino beam. The detectors and beamline facility are briefly discussed in Chapter 2. The properties of neutrino interactions in the MINOS detectors are the subject of Chapter 3 ; this chapter also describes the techniques used to reconstruct the neutrino interactions. Chapters 4 and 5 present the new methods used to identify muons and measure the muon charge sign in the MINOS detectors. The near detector data 


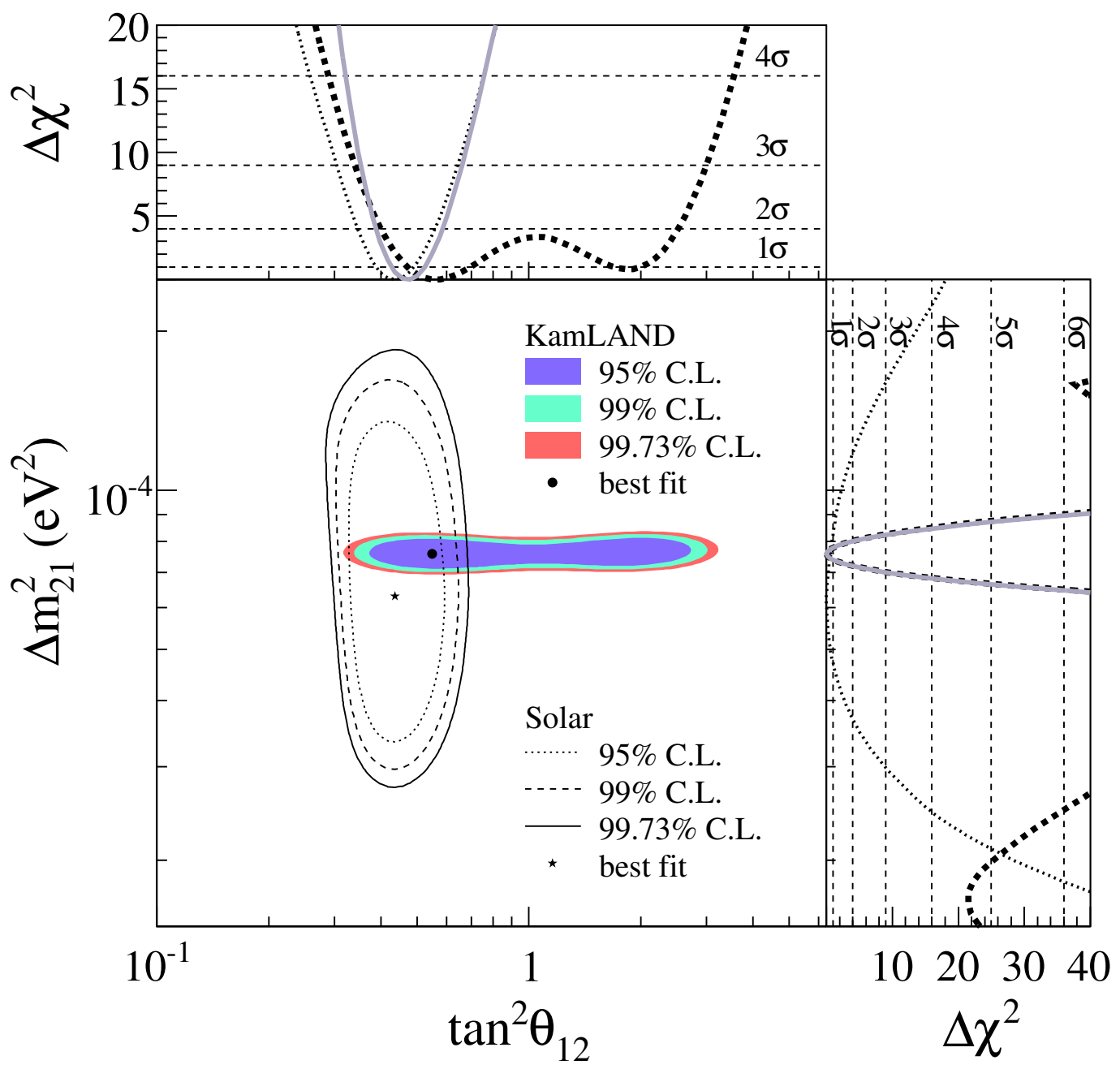

Figure 1.9: Allowed region for the neutrino oscillation parameters from the KamLAND and solar neutrino experiments. The side-panels show the $\Delta \chi^{2}$ profiles for KamLAND (dashed) and solar experiments (dotted) individually, as well as the combination of the two (solid). 


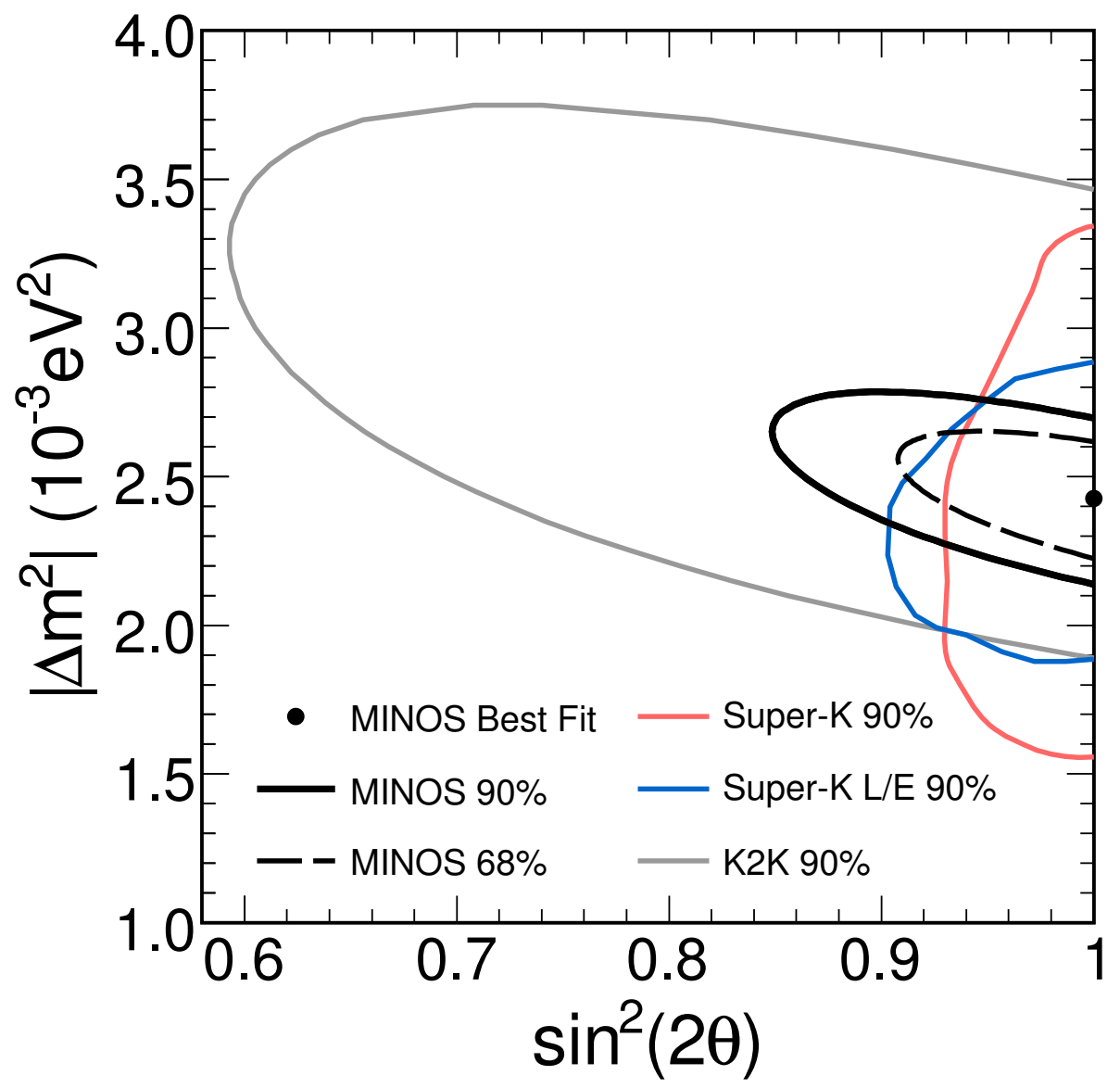

Figure 1.10: Figure shows $68 \%$ and $90 \%$ C.L. regions from the MINOS, SuperKamiokande, and $\mathrm{K} 2 \mathrm{~K}$ experiments for the measurement of oscillation parameters [46]. The best fit parameters for the published MINOS result are: $\sin ^{2} 2 \theta>0.90$ (90\% C.L.) and $\left|\Delta m^{2}\right|=(2.43 \pm 0.13) \times 10^{-3} \mathrm{eV}^{2}(68 \%$ C.L $)$ (statical and systematic errors combined). 
are analyzed in Chapters 6 and 7. This near detector analysis computes corrections to neutrino flux and cross-section models. These corrections are used in Chapter 8 to compute a predicted event rate at the far detector assuming there are no oscillations. Chapter 9 measures oscillation parameters with the far detector data. 


\section{Chapter 2}

\section{NuMI facility and MINOS detectors}

The Main Injector Neutrino Oscillation Search (MINOS) experiment [52] is a two-detector neutrino experiment that uses an intense muon neutrino beam to study neutrino oscillations. The muon neutrino beam is generated by the Neutrinos at the Main Injector (NuMI) facility [53] at Fermi National Accelerator Laboratory (Fermilab), and it is aimed in the direction of the Soudan Underground Laboratory in Northern Minnesota. This chapter briefly describes the design and operation of the NuMI facility and the MINOS detectors.

\section{$2.1 \quad$ Neutrino beams}

An idea for neutrino experiments using accelerators was proposed independently by Pontecorvo [54] and Schwartz [55] around 1960. This idea led to the first accelerator based neutrino experiment at Brookhaven AGS and the discovery of a muon neutrino [10].

A muon neutrino beam is generated by the decay of pions and kaons:

$$
\begin{array}{r}
\pi^{ \pm} \rightarrow \mu^{ \pm}+\nu_{\mu}\left(\bar{\nu}_{\mu}\right) \\
K^{ \pm} \rightarrow \mu^{ \pm}+\nu_{\mu}\left(\bar{\nu}_{\mu}\right)
\end{array}
$$

The pions and kaons are produced by interactions of high energy protons with 
a target. The pions emit neutrinos with energies up to $43 \%$ of the pion energy and in a direction frequently aligned with the pion direction. A neutrino detector is placed behind shielding material, which stops remaining primary protons, secondary hadrons, and tertiary muons. Charged pions can be directed forward using a focusing magnet (horn) $[55,56]$, which produces a more intense collimated neutrino beam.

\section{$2.2 \quad$ NuMI facility}

The NuMI is a neutrino beamline facility $[53,57,58]$ constructed at the Fermi National Accelerator Laboratory in Batavia, Illinois. The construction of the NuMI facility was completed in Winter 2005, and routine operations began in May, 2005. The NuMI operations are projected to continue through the next decade.

Protons with a $120 \mathrm{GeV} / \mathrm{c}$ momentum are extracted from the Main Injector (MI) accelerator in a single pulse (approximately $10 \mu$ s long) and are transported $350 \mathrm{~m}$ to the NuMI target. A transport line bends the proton pulses downward 58 mrad toward Soudan, Minnesota (see Figure 2.1). The extractions typically contain $2.1-3.1 \times 10^{12}$ protons with a cycle time of $2.2-2.4$ s. Figure 2.2 summarizes the number of protons delivered to the NuMI target since the start of the NuMI operations.

The proton pulses are focused onto a $94 \mathrm{~cm}$ long graphite target made of 47 longitudinal segments. The segmented design was chosen to relieve thermal stresses from high energy proton collisions. The target is continuously 
water cooled and is enclosed within an aluminum vacuum vessel with beryllium windows. A $1.5 \mathrm{~m}$ long graphite baffle with an $11 \mathrm{~mm}$ inner bore diameter is installed upstream of the target (see Figure 2.3). The baffle protects the target, target assembly, and focusing magnets from misdirected proton pulses.

Two focusing magnets (horns) are positioned downstream of the target and are spaced $10 \mathrm{~m}$ apart (see Figure 2.3). The horns have parabolic inner conductors acting as a "lens," with the focal length for point-to-parallel focusing proportional to particle momentum. The horns are pulsed simultaneously with a proton pulse reaching a $200 \mathrm{kA}$ peak current and generating a maximum 30 kG magnetic field. The NuMI horns are pulsed in "forward" polarity, focusing positively charged secondary hadrons.

The target and baffle are mounted on a rail system, allowing longitudinal motion within a $2.5 \mathrm{~m}$ range. The relative distance between the target and horns determines a range of hadron momenta, which are preferentially focused in the forward direction. Figure 2.4 shows energy distributions for $\nu_{\mu}$ charged-current events in the near detector for the 3 target positions (beam configurations). Data from the $\mathrm{L} 10 \mathrm{~cm} / 185 \mathrm{kA}$ configuration are used for the oscillation analysis in Chapter 9. Data from other beam configurations are used to tune neutrino flux and cross-section models, as described in Chapter 7 . 

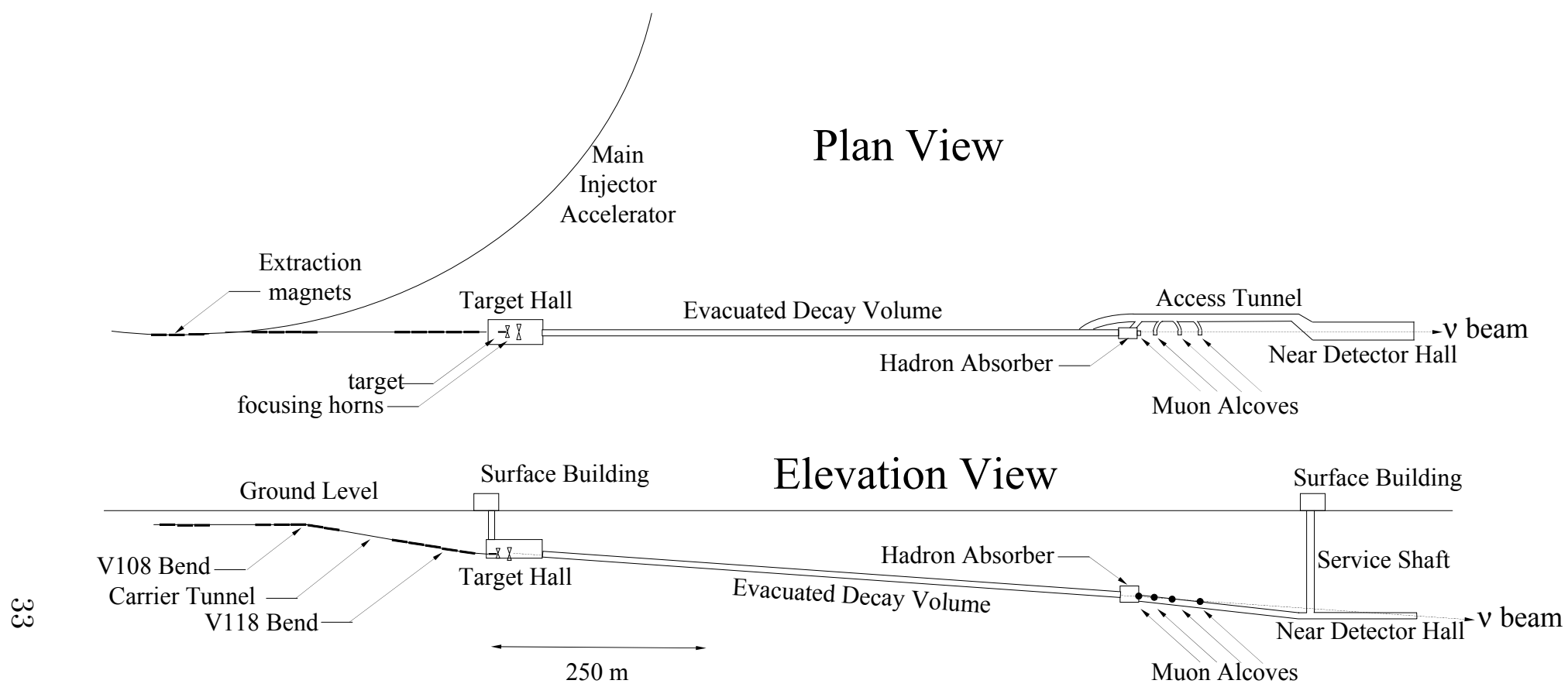

Figure 2.1: Schematic view of the NuMI neutrino beam facility. A proton beam is extracted from the Main Injector and is directed onto a graphite target. Secondary pions and kaons are focused by magnetic horns (Figure 2.3) into a decay volume. Ionization chambers, placed in front of hadron absorber and in muon alcoves, measure the flux of secondary hadrons and tertiary muons. This figure was taken from [58]. 


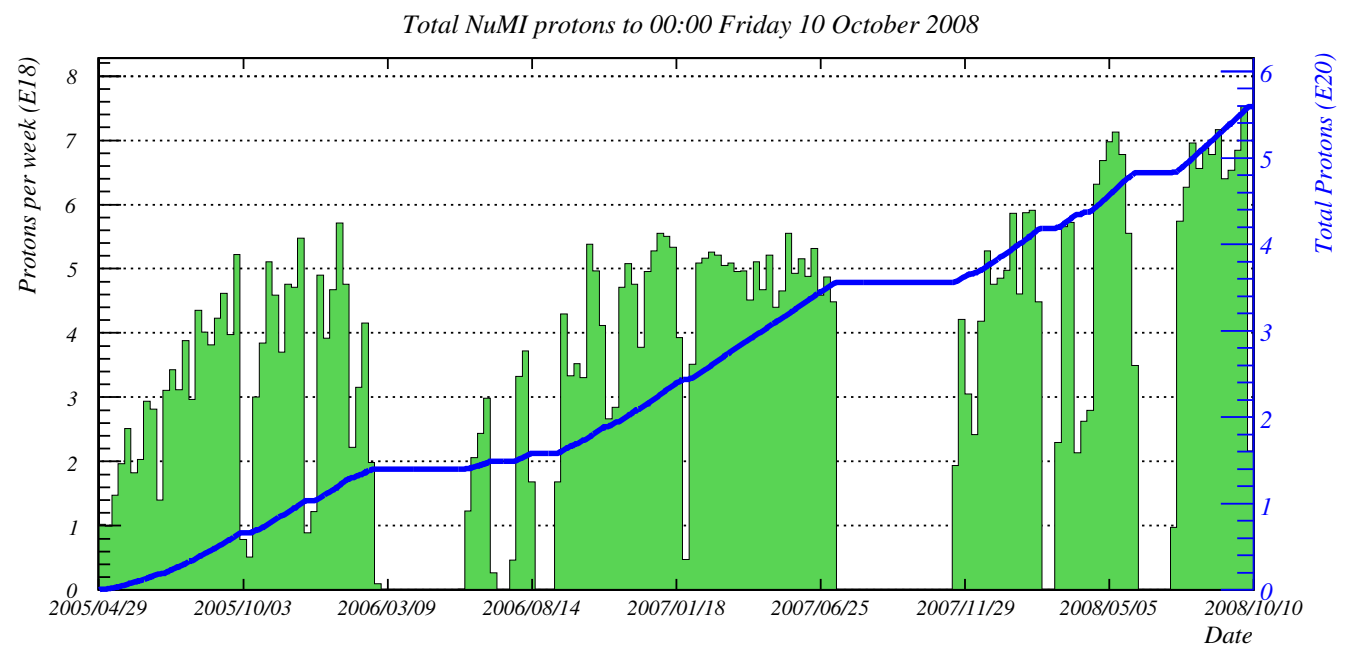

Figure 2.2: Number of protons on a target (POT) delivered to the NuMI target. The solid green histogram (using the left vertical axis) shows an average number of POT per week plotted versus time. The blue line (using the right vertical axis) shows an integrated number of POT. The thesis analysis uses the near detector data recorded between May, 2005 and July, 2007.

The horns focus secondary hadrons into a $675 \mathrm{~m}$ long, $2 \mathrm{~m}$ diameter "decay" pipe. The decay pipe is made of steel and surrounded by $2.5-3.5 \mathrm{~m}$ of concrete to prevent activation of ground water and soil. An entrance window to the decay pipe is made of steel and aluminum. An absorber, made of aluminum, steel, and concrete, is located at the end of the decay pipe. The absorber and walls of the decay pipe are water cooled to remove the heat deposited by secondary hadrons. Initially, the decay pipe was evacuated to 1 Torr. In Fall 2007, the decay pipe was filled with helium at the atmospheric pressure due to concerns about the integrity of the entrance window. This thesis analysis uses the data recorded between May, 2005 and July, 2007. 


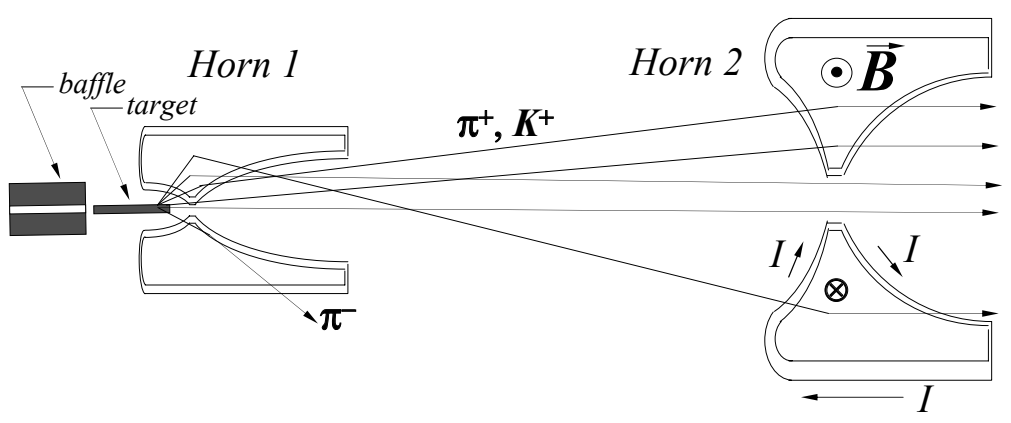

Figure 2.3: Drawing of a baffle, a target, and two magnetic horns (separated by $10 \mathrm{~m}$ ). The NuMI horns are pulsed in "forward" polarity, focusing positively charged secondary hadrons. The baffle and target can be moved together further upstream (to the left) of the horns to focus higher momentum secondary hadrons and to produce a higher energy neutrino beam (see Figure 2.4). The vertical scale is 4 times greater than the horizontal scale. This figure was taken from [58].

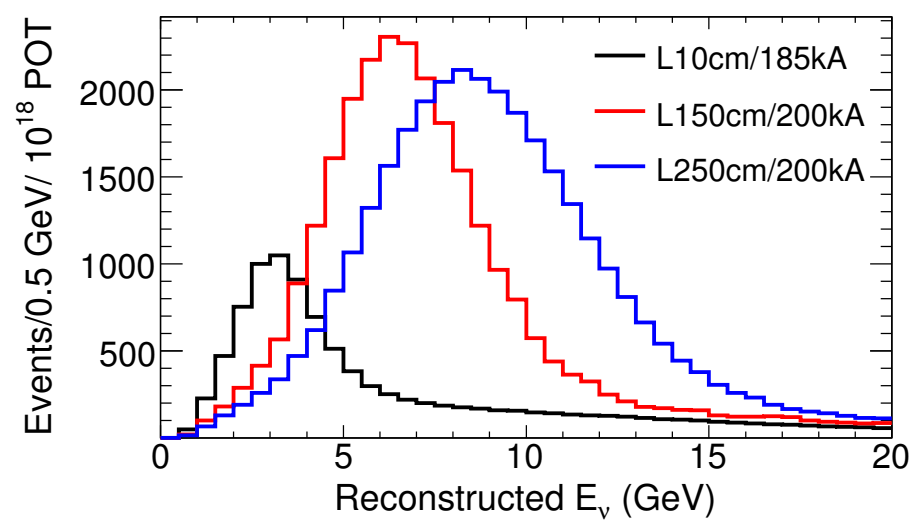

Figure 2.4: Reconstructed neutrino energy distributions in the MINOS near detector. The figure shows the $\nu_{\mu}$ charged-current data events (discussed in Chapters 4 and 6), recorded using 3 target positions, with the target placed $10 \mathrm{~cm}, 150 \mathrm{~cm}$ and $250 \mathrm{~cm}$ away from the 1st horn (see Figure 2.3). The horns are pulsed at $185 \mathrm{kA}$ or $200 \mathrm{kA}$ current, producing a maximum $30 \mathrm{kG}$ toroidal magnetic field. The number of events is normalized to $10^{18}$ protons on target (POT). 
The transport line from the Main Injector to the target is instrumented with beam position monitors and beam profile monitors. The beam trajectory and beam profile are monitored for every proton beam pulse (spill). Proton pulse intensity is measured using two toroidal beam current monitors (toroids) and loss monitors. The current flowing through the horns is recorded for every pulse. Less than $1 \%$ of the proton pulses with parameters outside an accepted window are rejected from analysis [59]. The accepted window was defined to select proton pulses with charatestics as similar as possible to the NuMI design specifications.

The physics goals of the MINOS experiment require an accurate alignment of the proton beam and the MINOS far detector. The absolute positions of the NuMI facility and Soudan underground lab were measured using GPS satellites and an inertial survey. The inertial survey used accelerometers and optical gyroscopes to establish a global coordinate system for the underground detector hall. The relative positions of the NuMI target and far detector were established with $0.7 \mathrm{~m}$ per coordinate precision [52]. An independent check indicated that the neutrino beam is aimed at the far detector within $20 \mathrm{~m}$ [52]. This systematic error introduces a negligible uncertainty into the calculation of muon neutrino flux at the far detector [58].

A Monte-Carlo (MC) simulation is used to predict muon neutrino flux at the near and far detectors. The MC simulation of the NuMI neutrino flux is done in two steps. First, the FLUKA05 program [60,61] is used to simulate interactions of the $120 \mathrm{GeV}$ protons with the NuMI target (hadron production 
simulation). These interactions produce pions and kaons that are passed on to the next simulation step. These pions and kaons are propagated through the magnetic horns and are allowed to decay within the decay pipe (beamline simulation). The beamline simulation uses the GEANT3 program [62].

Figure 2.5 shows the contributions to the predicted MC neutrino spectrum at the near detector from the pion and kaon decays for different beam configurations. A beam configuration is identified by the relative position of the two magnetic horns, the target position, and the current pulsed through the horns. For example, the low energy (LE) beam configuration is identified as "L10cm/185kA." In this name, L stands for the (low energy) position of the two horns ${ }^{1}$ and $10 \mathrm{~cm}$ is the position of the target relative to the 1st horn and $185 \mathrm{kA}$ is a current pulsed through the NuMI horns. In all beam configurations the predicted $\mathrm{MC}$ spectrum is dominated by neutrinos from the pion decays.

\section{$2.3 \quad$ MINOS detectors}

MINOS consists of two iron and scintillator detectors designed as hadronic sampling calorimeters with a muon tracking capability. A near detector is located at Fermilab, $104 \mathrm{~m}$ underground and $1040 \mathrm{~m}$ away from the NuMI target. A far detector is located in the Soudan mine, $705 \mathrm{~m}$ underground and $735.34 \mathrm{~km}$ away from the NuMI target. The two detectors are shown in

\footnotetext{
${ }^{1}$ The relative position of the NuMI horns has not changed since the start of the NuMI operations.
} 

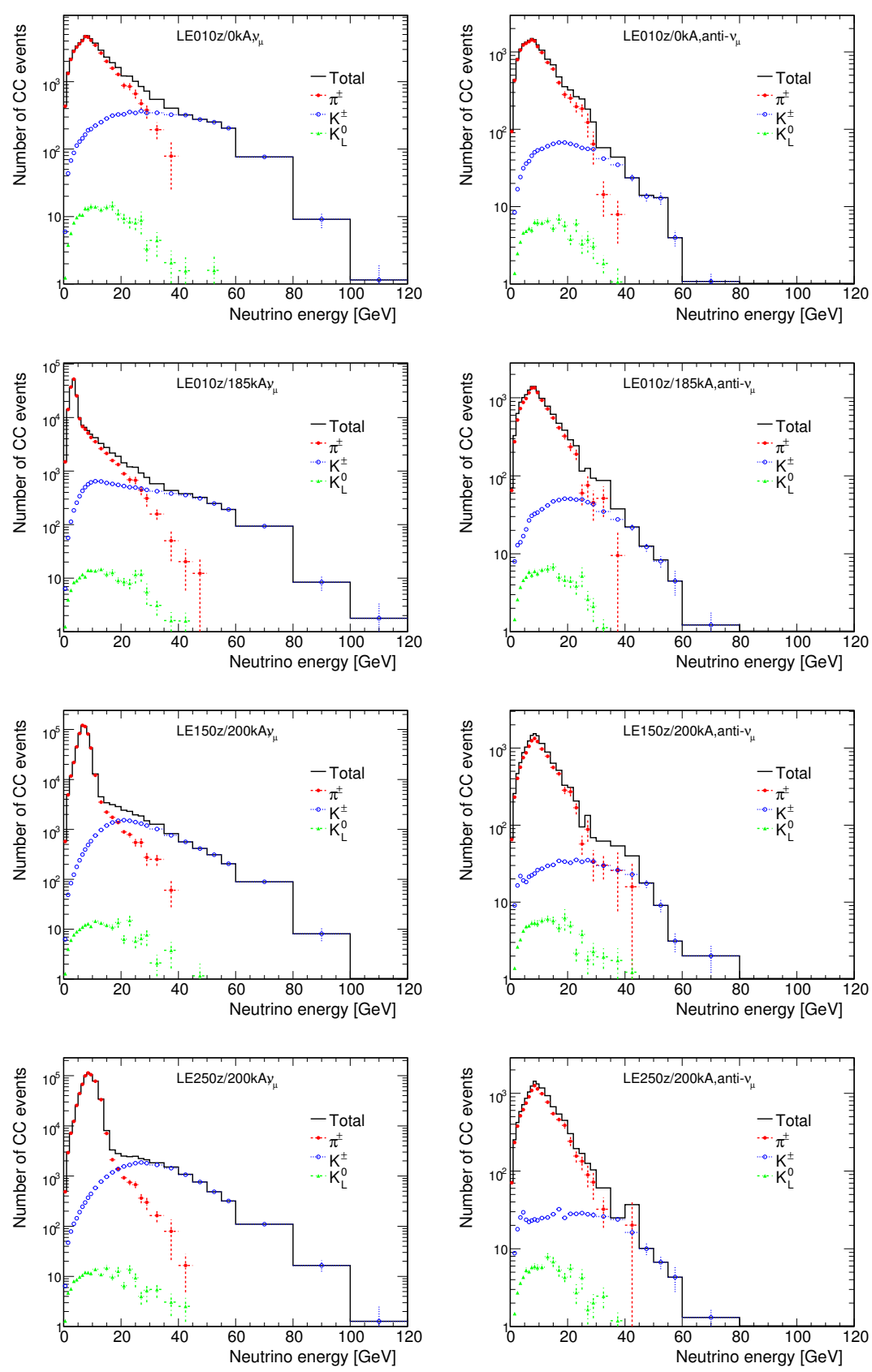

Figure 2.5: The left panels show contributions to the near detector $\nu_{\mu}$ chargedcurrent spectrum from $\pi^{+}$and $K^{+}$parents. The right panels show contributions to the near detector $\bar{\nu}_{\mu}$ charged-current spectrum from $\pi^{-}$and $K^{-}$ parents. The plots were taken from [63]. 
Figure 2.6. The two detectors were designed to provide a similar response to neutrino interactions while taking into account the difference in the neutrino interaction rate. ${ }^{2}$

The two detectors are constructed of alternating planes made of magnetized steel and plastic scintillator. The steel planes are $2.54 \mathrm{~cm}$ thick with different dimensions for the two detectors. The scintillator planes are made of $4.1 \mathrm{~cm}$ wide, $1.0 \mathrm{~cm}$ thick plastic scintillator strips, organized in modules of 20 or 28 strips. The modules are combined to form one scintillator plane, which is attached to a steel plane. The detectors were built as a series of steel and scintillator planes hung vertically and placed every $5.95 \mathrm{~cm}$ along a detector axis (see Figure 2.6). Scintillator strips in two successive planes are rotated by $90^{\circ}$ to allow two independent views of neutrino interactions. These two views are called the $\mathrm{U}$ and $\mathrm{V}$ views. A combination of these two views allows three-dimensional event reconstruction.

The scintillator strip technology was developed by the MINOS collaboration in a three-year research program [52]. The scintillator strips were made in an extrusion process. During the extrusion process, a groove for a wavelength shifting (WLS) fiber was also made, and a $0.25 \mathrm{~mm}$ thick $\mathrm{TiO}_{2}$ reflective layer was applied (see Figure 2.7). Charged particles passing through a scintillator strip generate scintillation light, which is absorbed by the WLS fiber and re-emitted isotropically. The re-emitted light is transported via WLS and

\footnotetext{
${ }^{2} \mathrm{~A}$ detailed description of the MINOS detectors is presented in Reference [52].
} 


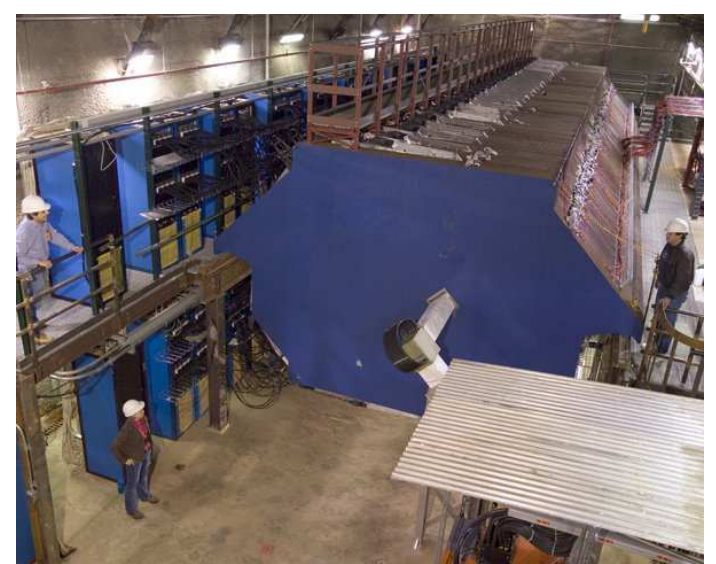

(a)

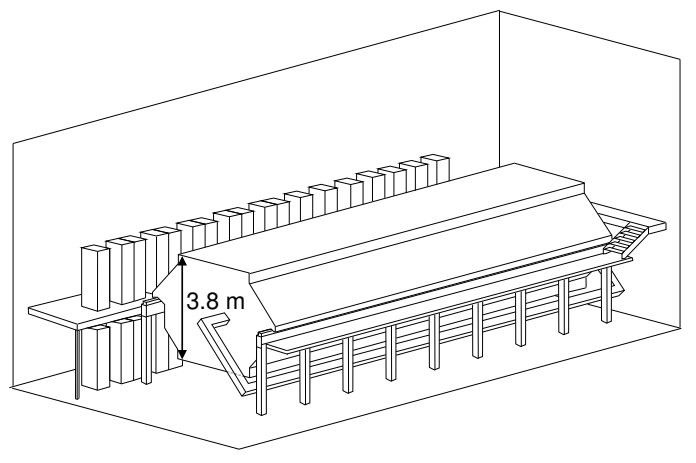

(c)

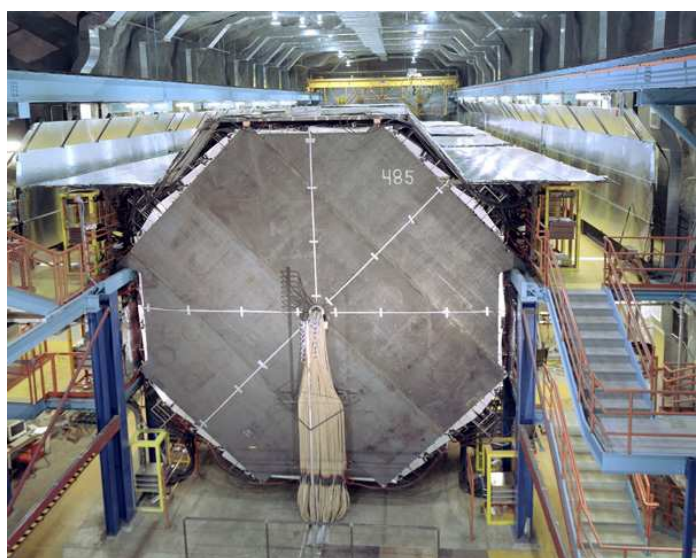

(b)

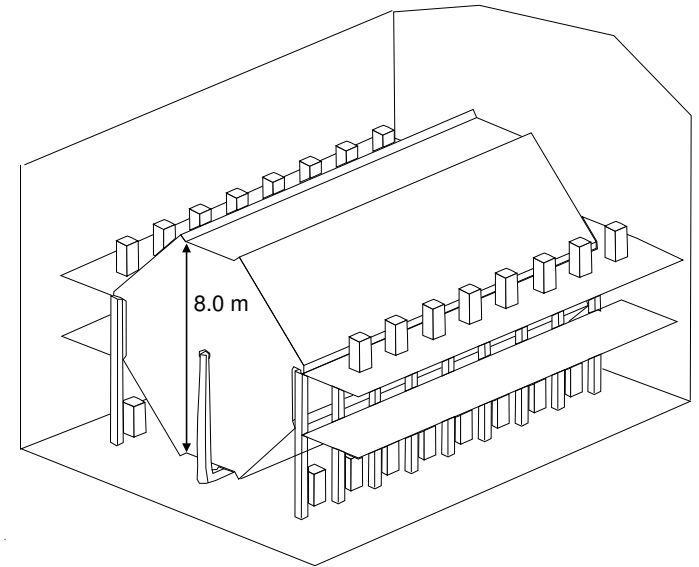

(d)

Figure 2.6: (a) A view of the MINOS near detector looking toward Soudan. (b) A view of the MINOS far detector looking toward Fermilab. These two images are courtesy of the Fermilab Visual Media Services. (c) A schematic drawing of the MINOS near detector. (d) A schematic drawing of the MINOS far detector. The far detector consists of two functionally identical modules, only one of the modules is shown in the figure. The drawings were taken from [58]. 
clear fibers to a photomultiplier tube (PMT), as illustrated in Figure 2.8.

The near detector has a mass of 980 metric ton and consists of 282 steel planes and 153 scintillator planes. Each steel plane is a $6.2 \mathrm{~m}$ wide, $3.8 \mathrm{~m}$ high "squashed octagon." The near detector is divided into two sections: the calorimeter and the spectrometer. The calorimeter consists of 121 steel and 120 scintillator upstream planes, and it is used to measure event topology, shower energy, and muon momentum. A fiducial volume is located entirely within the calorimeter. The spectrometer consists of the remaining detector planes, and it is used exclusively for muon tracking. In the spectrometer, a scintillator plane is attached to every fifth steel plane. The near detector steel planes are magnetized using a $40 \mathrm{kA}$ coil current; this produces an average 1.17 $\mathrm{T}$ magnetic field in a fiducial volume.

The near detector uses two types of scintillator planes. Partially instrumented planes contain 64 scintillator strips connected to a single PMT. Fully instrumented planes contain 96 scintillator strips connected to two PMTs. The near detector uses Hamamatsu 64-anode PMTs. Scintillator strips are connected to a PMT using a clear fiber connected to a WLS fiber from one strip-end; a WLS fiber on the opposite strip-end is covered with a mirror. In the calorimeter, every fifth scintillator plane is fully instrumented, and the remaining planes are partially instrumented. In the spectrometer, all scintillator planes are fully instrumented.

The far detector has a mass of 5400 metric tons and consists of 486 steel planes and 484 scintillator planes. Each steel plane is an $8.0 \mathrm{~m}$ wide regular 


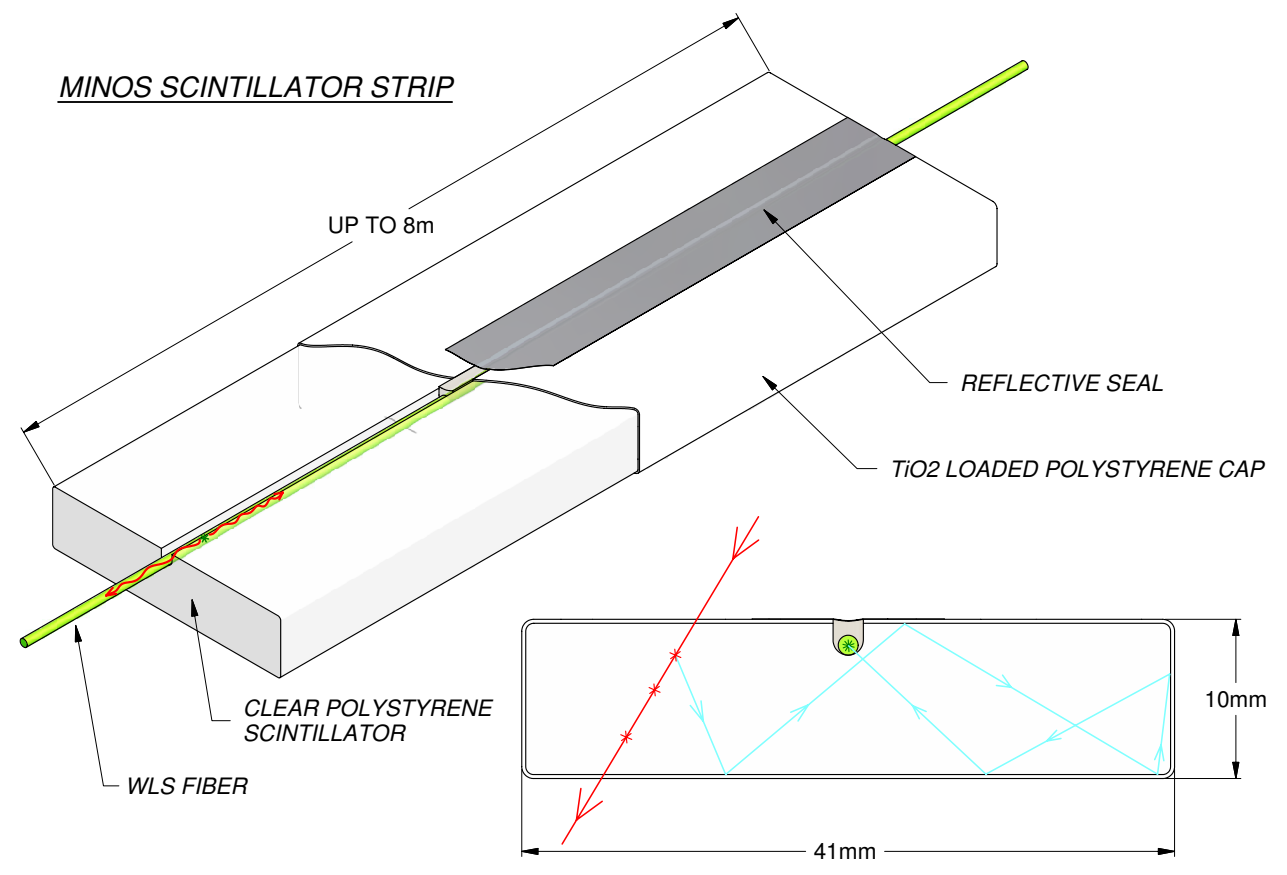

Figure 2.7: A cutaway drawing of a MINOS scintillator strip. Scintillation light is produced by an ionizing particle and is multiply reflected inside the strip by a $0.25 \mathrm{~mm}$ thick outer reflective layer (shown in the cross-section view). Light is absorbed by a wavelength shifting (WLS) fiber and is reemitted isotropically. The re-emitted photons, whose directions fall within the total internal reflection cones, are transported along the fiber to the strip's edges and routed to the photomultiplier tubes via clear fiber (see Figure 2.8). This figure was taken from [52]. 


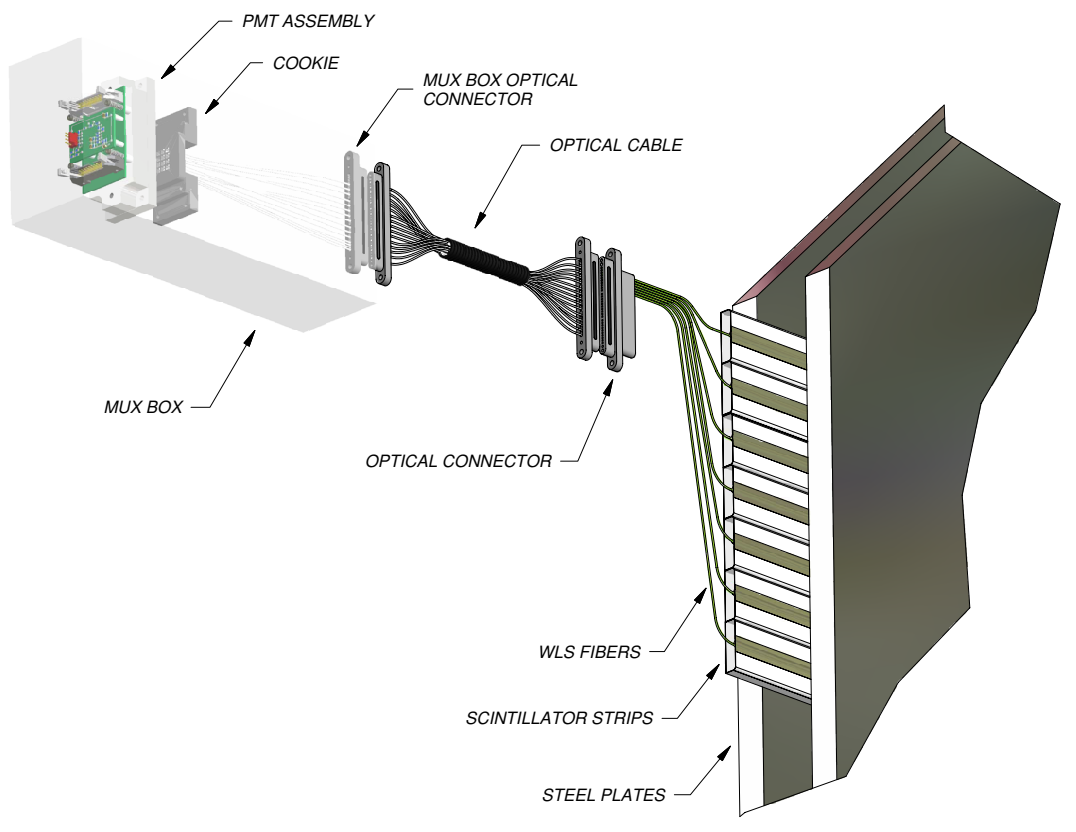

Figure 2.8: A schematic drawing of the optical readout for MINOS scintillator strips. The edges of two steel planes are on the right side of the drawing, showing several scintillator strips extending beyond the edges of the steel planes for clarity. Scintillation light is produced in a strip (see Figure 2.7) and travels out of the strip via WLS fiber. The WLS fiber is connected to a clear optical fiber, assembled into an optical cable and passing through two connectors, which guides the light to a photomultiplier tube. This figure was taken from [52]. 
octagon. The far detector is divided into two modules separated by a $1.1 \mathrm{~m}$ air gap. Scintillator planes contain 192 scintillator strips, with varying strip length. The scintillator strips are read out from both strip-ends through clear fibers connected to Hamamatsu 16 anode PMTs. In each scintillator plane, groups of 8 scintillator strips are multiplexed to a single PMT channel. Offline reconstruction algorithms use hit patterns and signals from both strip-ends to resolve multiplexing ambiguities [52]. The far detector steel planes are magnetized using a $15.2 \mathrm{kA}$ coil current, producing an average $1.27 \mathrm{~T}$ magnetic field.

The MINOS readout electronics were designed to provide adequate information for the classification of neutrino events and to enable an accurate measurement of event energy. The electronics were required to digitize the charge of each pulse from the PMTs with a dynamic range around 12-13 bits. The two detectors use different electronics systems to achieve this goal because the far detector observes approximately $10^{6}$ fewer neutrino interactions than the near detector, due to a divergence of the neutrino beam. A small calibration detector [64] was instrumented with the near and far electronics and PMTs. This detector was exposed to test beams at CERN, and the results of the tests showed that a calibrated response of the near and far detector readout systems are equal [65].

The near detector observes up to 20 fully contained events per spill. A timing accuracy of around $20 \mathrm{~ns}$ is sufficient for identification of individual events. The near detector electronics [66] continuously record signals from 
all detector channels during a neutrino beam spill. A signal is read out in contiguous $18.83 \mathrm{~ns}$ time intervals ${ }^{3}$ without dead-time for up to a $18 \mu$ s window. Any time interval with a signal greater than approximately 0.3 photoelectrons is digitized and passed to a data acquisition system.

The far detector electronics [67] were designed for a low rate underground environment. The detector rate is dominated by detector noise of few $\mathrm{kHz}$ per PMT. The far detector PMTs are organized in groups, with 36 PMTs (20 or 24 scintillator planes) in one group. All signals from a group of 36 PMTs are digitized if there is a dynode signal exceeding approximately 0.25 photoelectrons in at least 2 different PMTs within a 400 ns window. This condition significantly reduces the detector noise rate without compromising the recording of physical events. A digitized signal over a programmable sparsification threshold is passed to a data acquisition system.

The data acquisition systems (DAQ) [68] for the two detectors are functionally identical and utilize off-the-shelf PCs running GNU/Linux operating system. The timing systems in the two detectors are synchronized using GPS satellites. The DAQ permanently records detector signals (hits) for an offline analysis if a trigger condition is met. Each recorded hit contains information about the time, charge, and physical location. Several different triggers are implemented in the DAQ. For example, the cosmic muon trigger requires multiple plane hits within a fixed time window [52].

\footnotetext{
${ }^{3}$ This interval corresponds to $53.103 \mathrm{MHz}$ frequency of the Main Injector RF system.
} 
The near detector DAQ permanently records all detector hits within a spill window without any preconditions. The near detector timing system sends a "remote spill trigger" with the GPS time of each beam spill to the far detector via the Internet. The far detector DAQ has a long buffer to store data. The far detector DAQ permanently records all detector hits within at least a $100 \mu$ s window around the expected neutrino arrival time. This window is wide enough to collect all the beam neutrino events with sufficient safety margins. The efficiency of the remote spill trigger is around $99 \%$.

\subsection{Detector calibration}

A precise measurement of the oscillation parameters requires accurate calibration of two spatially separated detectors. The MINOS detectors are sampling calorimeters. The near and far detectors use steel planes of equal thickness, produced by a single steel foundry. The two detectors also use the same scintillator strips, WLS fiber, and clear fiber. As a result, the two detectors have an equivalent response to hadronic and electromagnetic showers. A detector response is calibrated using an LED based light injection system [69], a calibration detector [64], a test-bench scan of the scintillator modules using a radioactive source [52], and cosmic ray muons $[70,71] .^{4}$

A signal is calibrated with a series of multiplicative factors that convert the raw photomultiplier signal $Q_{\text {raw }}(i, t, x)$ measured in channel $i$ at time $t$ for

\footnotetext{
${ }^{4} \mathrm{~A}$ detailed description of the detector calibration scheme is presented in [52].
} 
an energy deposition at position $x$ into the calibrated signal $Q_{\text {cal }}$ [52]:

$$
Q_{\text {cal }}=Q_{\text {raw }}(i, t, x) \times D(t) \times L\left(i, Q_{\text {raw }}\right) \times U(i, t) \times A(i, x) \times E
$$

where $D(t)$ corrects for the drifts in a channel response, $L\left(i, Q_{\text {raw }}\right)$ corrects for the non-linear channel response, $U(i, t)$ corrects for the non-uniform channel to channel response and $A(i, x)$ corrects for the fiber attenuation. $E$ is the overall scale factor that converts the fully corrected signal to an absolute energy unit common for the two detectors. Each detector uses an independent set of correction factors computed in-situ (except for the attenuation corrections). Figure 2.9 illustrates stages of the calibration process.

The drift correction $D(t)$ is computed using an overall detector response to through-going cosmic ray muons. This correction factor includes a drift of the entire optical system (caused by scintillator and fiber aging and drifts of PMT gains) and a drift in electronics response. The detector response varies with time at a level of a few percent [52].

The MINOS PMTs are 5-10\% nonlinear at approximately 100 photoelectrons. The linearity correction $L\left(i, Q_{\text {raw }}\right)$ is computed using the LI system. Each individual channel is pulsed at multiple light levels, and a channel response is compared to an independent linear measurement of light intensities [69].

The uniformity correction $U(i, t)$ uses through-going cosmic ray muons to normalize a mean response of individual scintillator strips to an average detector response. This coefficient corrects for a number of detector effects 
that vary from channel to channel: scintillator light yield, light collection efficiency of WLS fibers, clear fiber attenuation, PMT quantum efficiency, and PMT gain. The response of individual strips varies by approximately $30 \%$, compared with the mean detector response [52].

The attenuation correction $A(i, x)$ of the WLS fibers was measured using a test-stand and was checked in-situ using through-going cosmic ray muons. During the construction of the scintillator modules, a radioactive source was used to map out a longitudinal response of the scintillator strips in each module [52]. The response of each scintillator strip was parametrized with an empirical formula. The resulting parametrization is used to correct a signal of muon and shower energy depositions based on a longitudinal position of a muon or shower hit. In the near detector, the attenuation corrections vary by approximately $50 \%$ for a $3 \mathrm{~m}$ scintillator strip. In the far detector, the attenution corrections vary by approximately $30 \%$ for an $8 \mathrm{~m}$ scintillator strip (for a sum of signals from both strip-ends).

The overall energy scale is determined from the detector response to muons with a known momentum, using cosmic ray muons that stop within a detector. A momentum of stopping muons is measured with $2 \%$ uncertainty using a range of the muon track within the detector. Figure 2.10 shows a detector response plotted as a function of the muon momentum for the data and Monte-Carlo simulation. The overall energy scale normalizes the relative response of the near, far, and calibration detectors to within approximately $2 \%[52]$. 


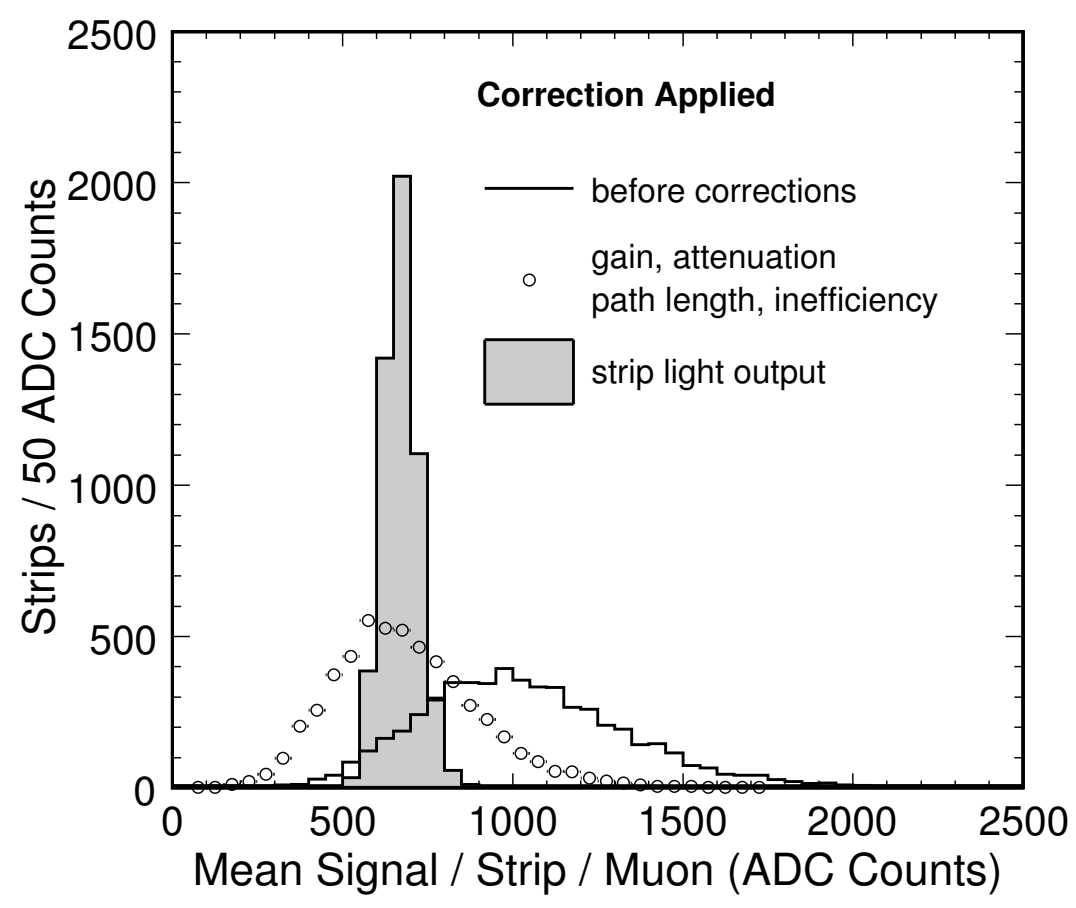

Figure 2.9: Raw and calibrated mean scintillator strip signal induced by muons produced in beam neutrino interactions in the near detector. The continuous line shows the raw signal before any corrections. Open circles show the signal with corrections accounting for variations in channel gain, attenuation in optical fiber, and strip light output. The solid histogram includes additional corrections for a non-uniform response of the scintillator and WLS fiber. This figure was taken from [52]. 


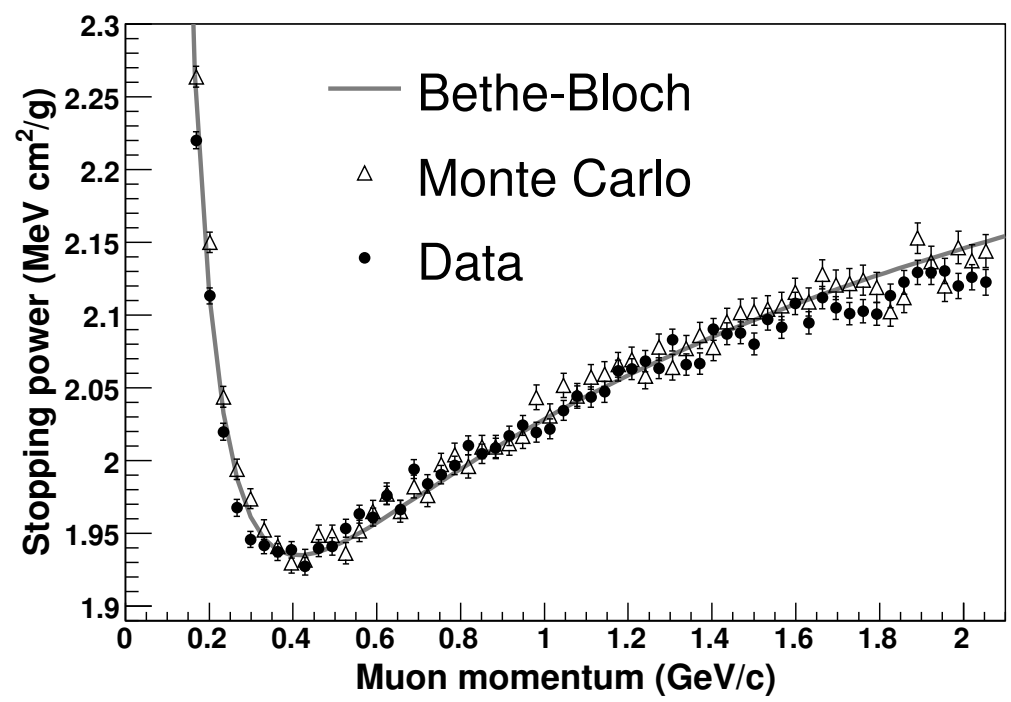

Figure 2.10: Absolute scintillator strip calibration using stopping power for muons. The gray line shows the Bethe-Block calculation [5] of the stopping power for muons in polystyrene scintillator. The solid circles and open triangles show the response stopping muons in the far detector data and the GEANT Monte-Carlo simulation, respectively. Both data and simulation are scaled to match the calculation at the minimum ionizing point. This figure was taken from [52]. 


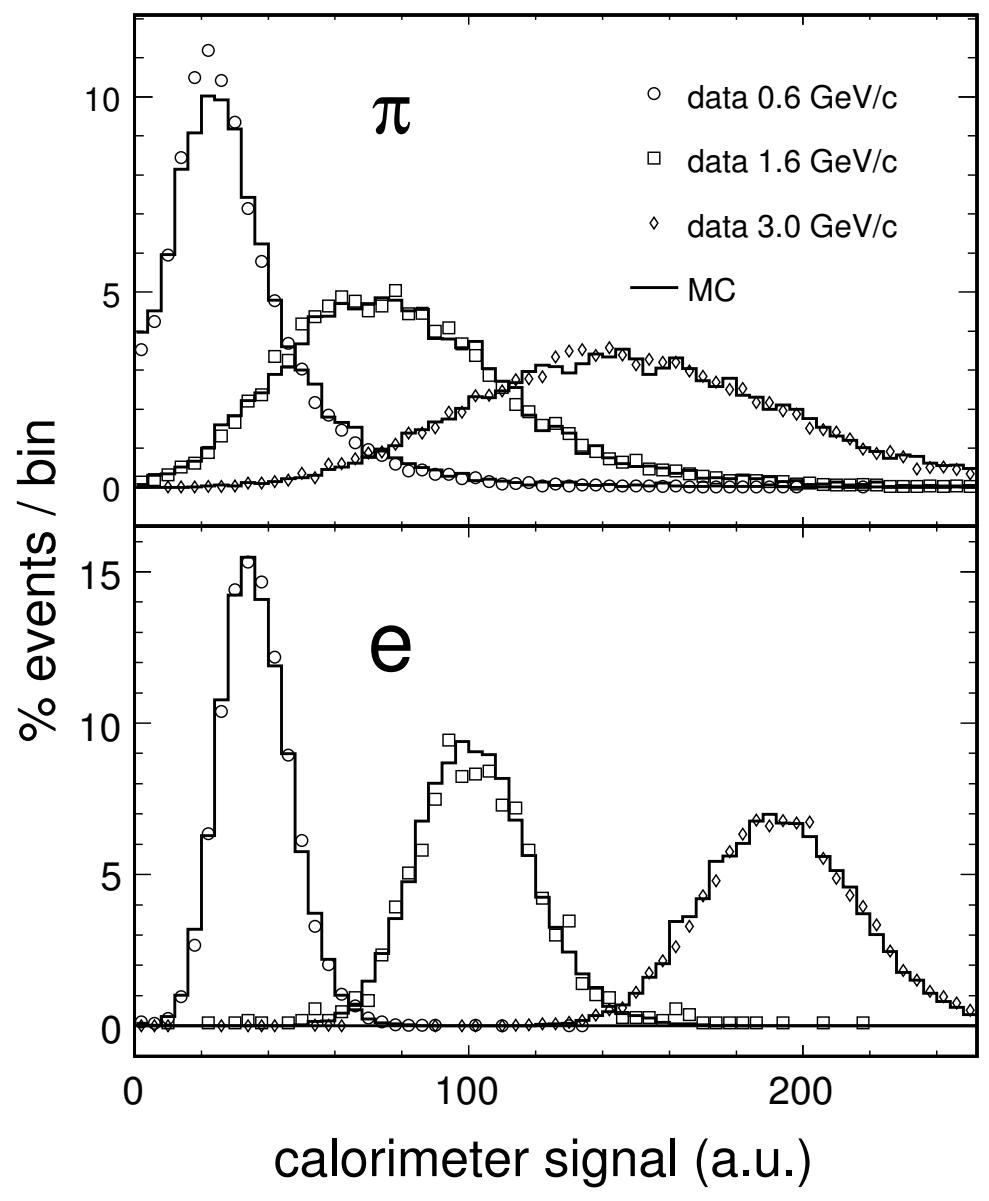

Figure 2.11: MINOS calorimetric response to pions and electrons at a three particle momenta. The calorimeter signal is measured in arbitrary units. The data (open symbols), obtained from the exposure of the calibration detector to CERN test beams, are compared with the Monte-Carlo simulation. This figure was taken from [52]. 
The calibration detector is a small copy of the MINOS detectors [64]. The calibration detector was used to establish an absolute energy scale calibration. It was exposed to muons, electrons, pions, and protons of known momentum in a test beam facility at CERN. These measurements were used to normalize a Monte-Carlo simulation to the test beam data and to establish $6 \%$ uncertainty on the absolute hadronic shower energy scale [52,72,73]. Figure 2.11 compares the CalDet measurements with the Monte-Carlo simulation. 


\section{Chapter 3}

\section{Neutrino interactions in the MINOS detectors}

This chapter examines the properties of neutrino interactions in the MINOS detectors. The neutrino interactions produce secondary particles; interactions of these secondary particles with the MINOS scintillator strips generate visible detector hits. These detectors hits are examined using reconstruction algorithms for patterns that match muon tracks and hadronic cascades (showers). The reconstruction algorithms create the reconstructed events; understanding these events is the subject of our analysis. Section 3.1 briefly describes the reconstruction algorithms used for analysis of the MINOS data.

Section 3.2 discusses the properties of $\nu_{\mu}$ charged-current interactions in the MINOS detectors. The total $\nu_{\mu}$ charged-current cross-section includes contributions from the quasielastic scattering (QES) process, the resonance production (RES) process, and the many additional channels collectively la-

beled as the deep inelastic scattering (DIS) process. These processes can be categorized by a multiplicity of hadronic final states in a hadronic cascade. Reconstructed hadronic showers in DIS, RES, and QES events are studied in Sections 3.3 and 3.4. These events are selected using the amount of the visible 
shower energy, as described in Section 3.3.

The MINOS collaboration developed a complete Monte-Carlo (MC) simulation model of the experiment. This model is discussed in Section 3.5. In general, the neutrino interactions are reasonably well understood, so the $\mathrm{MC}$ simulation is used to understand properties of neutrino interactions in the MINOS detector. In Section 3.6, detector hits from the data and MC simulation are examined to validate the MC simulation.

\subsection{Event reconstruction}

The event reconstruction procedure uses the topology and timing of detector hits to identify neutrino interactions inside the detector or surrounding rock. There are several types of neutrino interactions observed in the MINOS detectors: $\quad \nu_{\mathrm{e}}$ charged-current, $\nu_{\mu}$ charged-current, $\nu_{\tau}$ charged-current, and neutral-current. Figure 3.1 shows event displays for the typical $\nu_{\mu}$ chargedcurrent, neutral-current, and $\nu_{\mathrm{e}}$ charged-current interactions in the MINOS detectors. Only $\nu_{\mu}$ charged-current and neutral-current interactions contribute a significant event rate; the other types together contribute less than $1 \%$ of the events produced by the NuMI neutrino beam. A primary goal of the reconstruction procedure is to identify the $\nu_{\mu}$ charged-current and neutral-current interactions. In the $\nu_{\mu}$ charged-current interactions, a muon and a hadronic cascade are produced. A main component of the event reconstruction is a track finding algorithm, which identifies detector hits matching a muon track.

The track finding algorithm [74] relies on the following two assumptions 


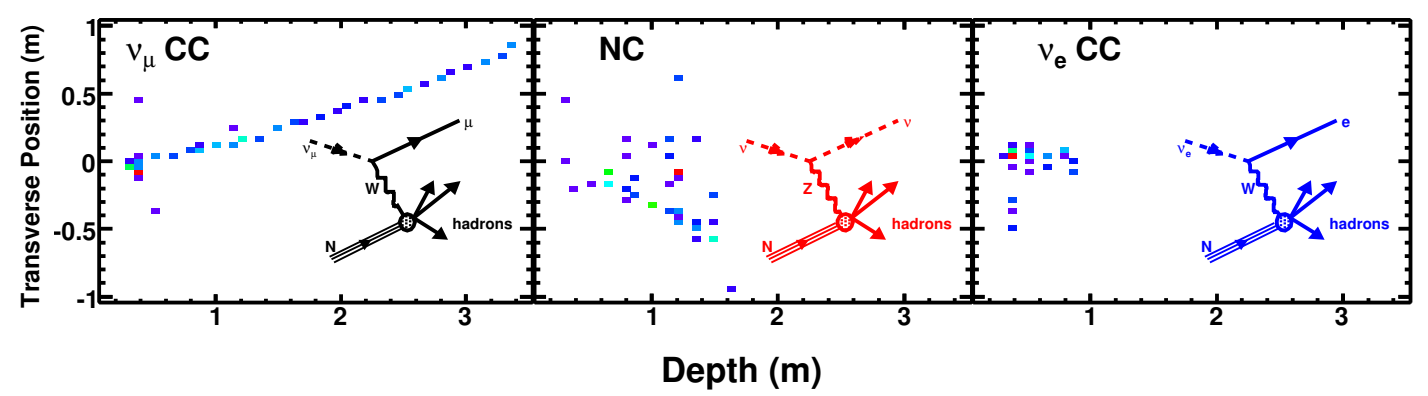

Figure 3.1: Examples of $\nu_{\mu}$ charged-current (left), neutral-current (center) and $\nu_{\mathrm{e}}$ charged-current (right) interactions in the MINOS detectors obtained from the MC simulation. The figures are courtesy of Patricia Vahle.

to find muons produced by the interaction of beam neutrinos: (a) muons preferentially travel along a detector axis; and (b) muons are very penetrating and cross multiple steel planes. First, the algorithm creates clusters; each cluster uses hits from a single plane with a pulse height greater than 2 photoelectrons. The clusters from neighboring planes are connected into segments. Then, the segments are chained together to create tracks: multiple arrangements of segments are examined to create a logically consistent track. The algorithm was developed to find any track-like patterns of hits with a minimum length of 6 detector planes. The algorithm finds almost $100 \%$ of muons with a momentum greater than $1 \mathrm{GeV}$, but the algorithm also frequently finds false muon tracks in neutral-current interactions. Chapter 4 of this dissertation presents a novel classification procedure that substantially reduces the number of false muon tracks in the MINOS detectors.

The track momentum is measured using a track range and track curvature. The track range through the detector material is integrated to obtain 
the muon momentum using range tables [75]. The muon momentum via the range is measured with the $2 \%$ uncertainty. The track curvature is measured using the track fitting algorithm [74]. This algorithm fits the track curvature using a Kalman filter technique, and it takes into account both bending of the muon track in the toroidal magnetic field, muon energy loss, and deflections from the multiple Coulomb scattering. The uncertainty of the muon momentum measurement via curvature is estimated using the stopping muon tracks by comparing the muon momentum via the range with the muon momentum via curvature. This method used to compute this systematic error is described in Section 6.1. The muon momentum via curvature is measured with the $3 \%$ uncertainty.

Showers are constructed from clusters of hits that are localized in space and time. The hadronic shower energy is estimated using the pulse height of the shower hits. If more than one shower is present in the event, the shower closest to a neutrino interaction vertex is used. The uncertainty on the absolute hadronic energy scale is $10.3 \%$, which is a sum in quadratures of 3 effects [46]: (a) the $8.3 \%$ uncertainty in the simulation of the neutrino interactions on iron nucleus; (b) the 5.5\% uncertainty in the calorimeter response to indvidual hadrons as derived in the test beam measurements [64]; (c) the $2.3 \%$ uncertainty in the energy scale calibration of the scintillator and readout systems.

Finally, the event reconstruction software combines these reconstructed tracks and showers into events. Reconstructed showers and tracks are assigned 
to a reconstructed event based on the start position of the track and shower and the timing of the track and shower hits. Data from the two detectors are analyzed using identical software algorithms. This analysis takes into account the individual geometry of each detector and the difference in the neutrino interaction rate.

An interaction vertex is the point at which a given neutrino interacts with the detector. For events that include a track, the vertex position is estimated using the starting point (vertex) of the track. The vertex positions of the selected $\nu_{\mu}$ charged-current events for the data and MC simulation are shown in Figures 3.2 and 3.3 for the near and far detectors, respectively. These figures show events recorded (simulated for the MC) with the target placed in the low energy configuration. The vertex positions are accurately modeled by the MC simulation (described in Section 3.5).

The reconstructed event energy, $E_{\nu}$, is:

$$
E_{\nu}=E_{h a d}+E_{\mu}
$$

where $E_{\text {had }}$ is the reconstructed shower energy and $P_{\mu}$ is the reconstructed muon track momentum. For tracks that stop within the detector, the muon momentum is measured via the range. Otherwise, the track momentum is measured via the curvature. The shower energy, muon momentum, and muon angle for the selected $\nu_{\mu}$ charged-current events for the data and MC simulation are shown in Figures 3.4 and 3.5 for the near and far detectors, respectively. The figures show events recorded (simulated for the MC) with the target placed 

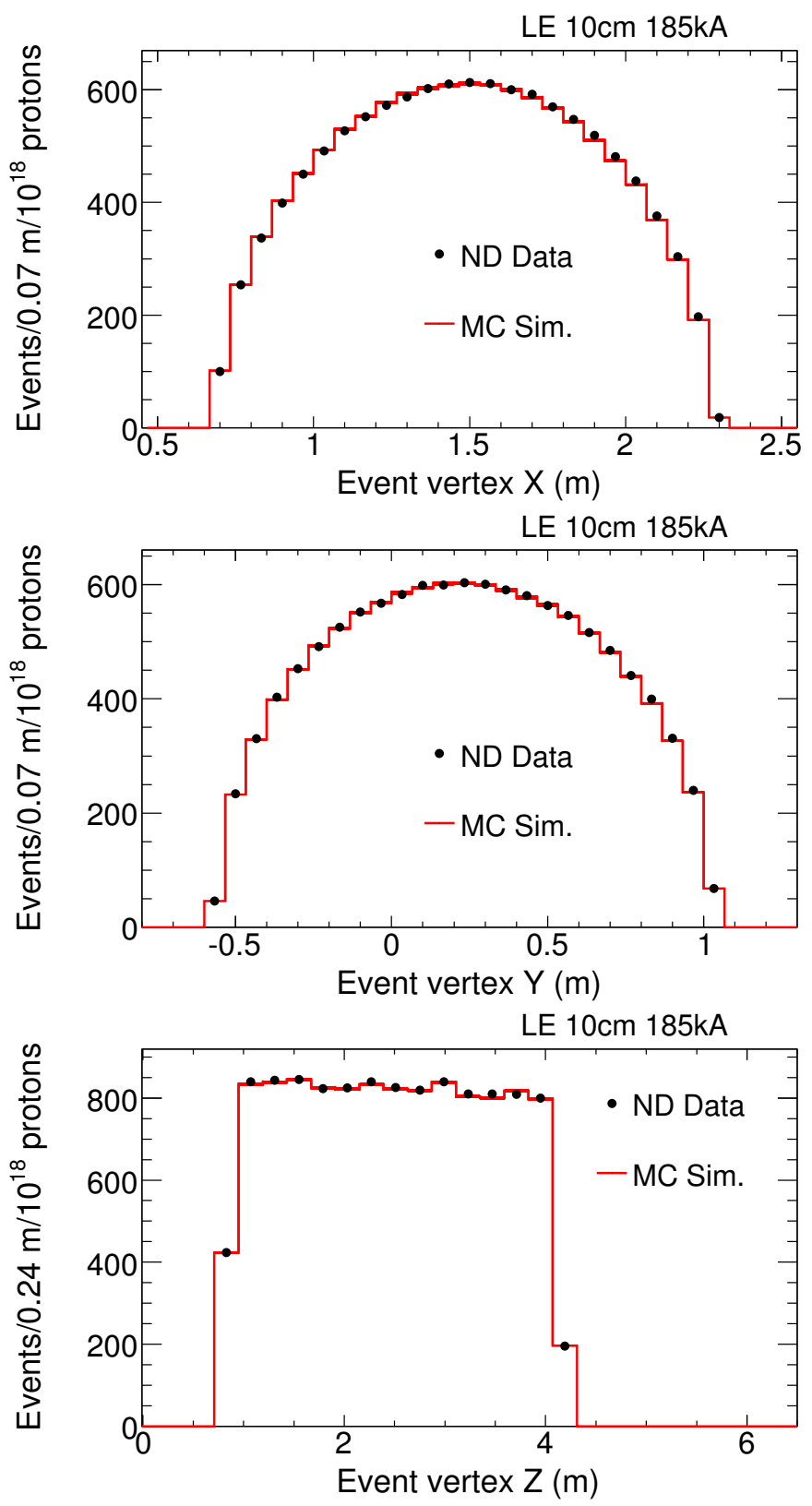

Figure 3.2: Reconstructed vertex positions for the selected $\nu_{\mu}$ charged-current events for the near detector data and MC simulation. The event selection procedure is described in Chapter 6. The MC simulation includes both flux and cross-section corrections computed in Chapter 7. The data events were recorded in the Run I period, as described in Section 6.3. 

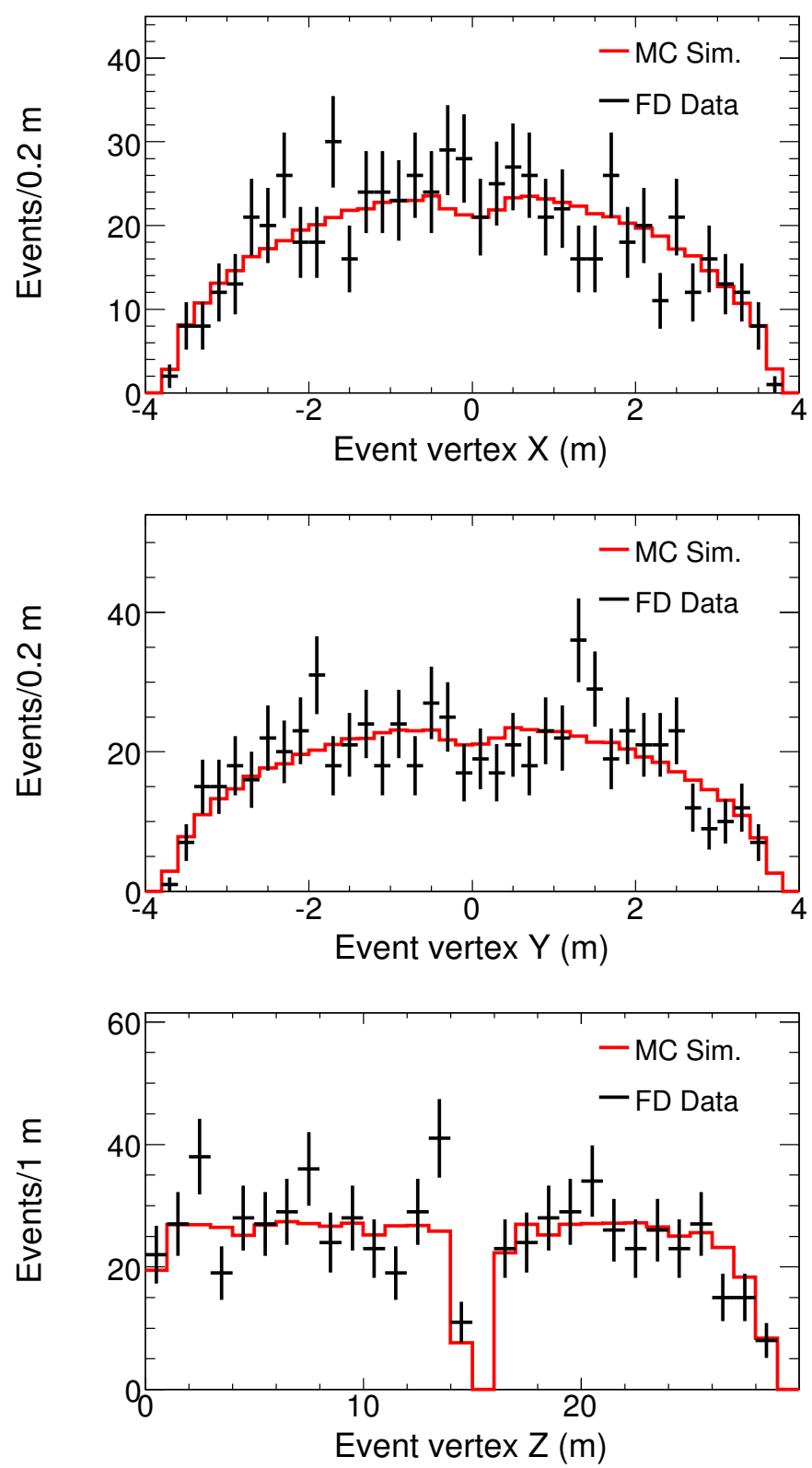

Figure 3.3: Reconstructed vertex positions for the selected $\nu_{\mu}$ charged-current events for the far detector data and MC simulation. The event selection procedure is described in Chapter 8. The MC simulation includes flux and crosssection corrections computed in Chapter 7 and corrections for the neutrino oscillations computed in Chapter 9. 
in the low energy configuration. These three reconstructed variables are accurately modeled by the MC simulation (described in Section 3.5).

In the near detector, hits that contain an electronics error code are excluded from the analysis. The remaining hits, with a signal greater than $40 \mathrm{ADC}^{1}$, are separated into time slices [76], and each slice is analyzed for tracks, showers, and events. The NuMI neutrino beam produces multiple neutrino interactions within the near detector. A use of time slices is required to separate overlapping events, illustrated in Figure 3.6. Figure 3.7 shows a mean number of the near detector events plotted as a function of the number of protons on target (POT). The number of events scales linearly with the number of POT. This plot shows that the overlapping events within the near detector are separated without intensity related errors.

In the far detector, a demultiplexing algorithm identifies which of the eight possible scintillator strips are associated with each far detector hit [77]. The algorithm compares signals from both strip-ends and uses timing and spatial information to resolve multiplexing ambiguities.

\subsection{Charged-current muon neutrino interactions}

The total $\nu_{\mu}$ charged-current cross-section includes contributions from three processes ${ }^{2}$ : quasielastic scattering (QES), resonance production (RES),

\footnotetext{
${ }^{1} 100 \mathrm{ADC} \approx 1$ photoelectron.

${ }^{2}$ Coherent pion production and flavor changing reactions do not contribute a significant event rate in the MINOS detectors [78]
} 

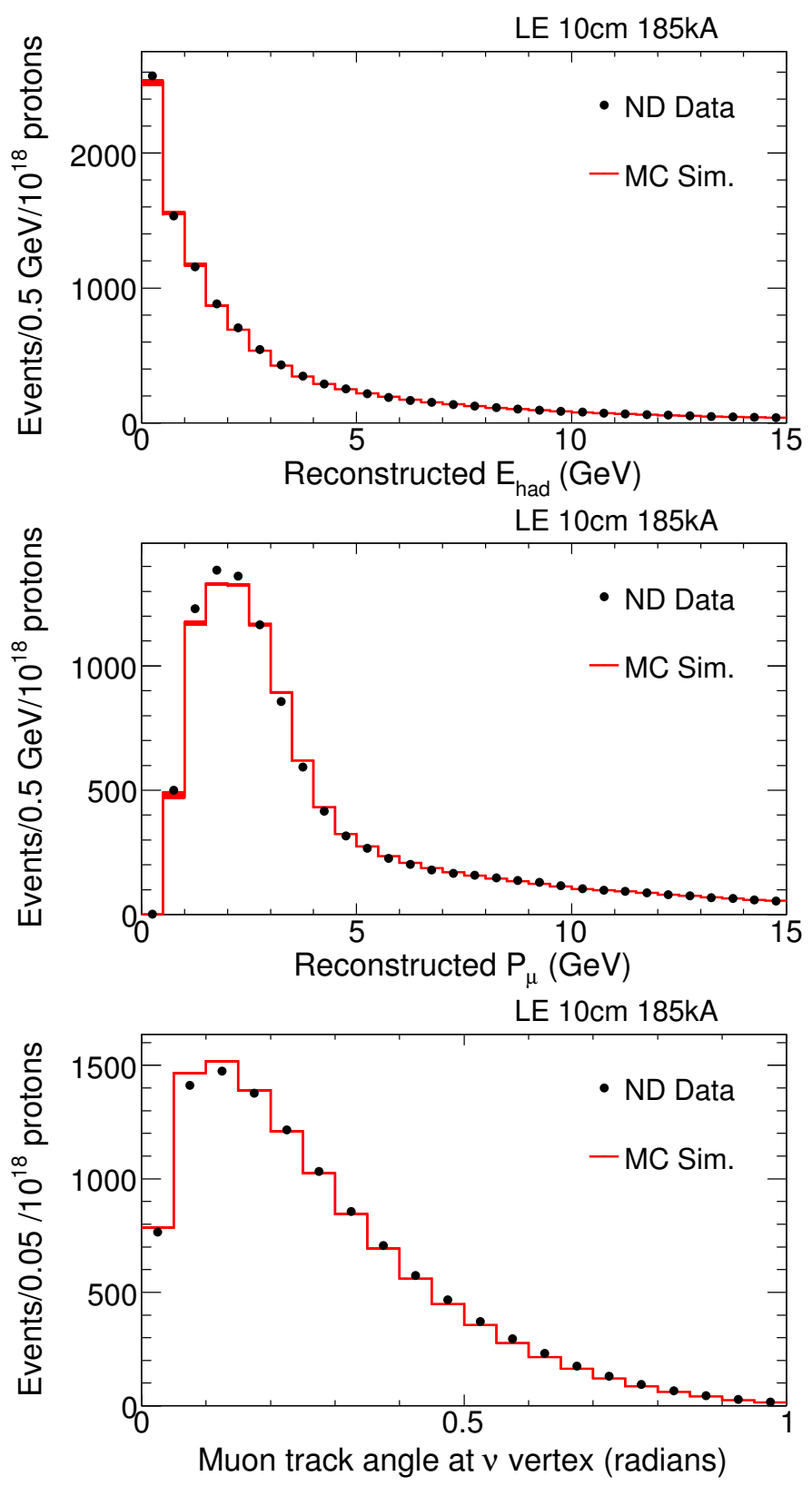

Figure 3.4: Reconstructed shower energy (top), muon momentum (middle), and muon angle (bottom) for the selected $\nu_{\mu}$ charged-current events for the near detector data and MC simulation. The event selection procedure is described in Chapter 6. The MC simulation includes flux and cross-section corrections computed in Chapter 7 . The data events were recorded in the Run I period, as described in Section 6.3. 

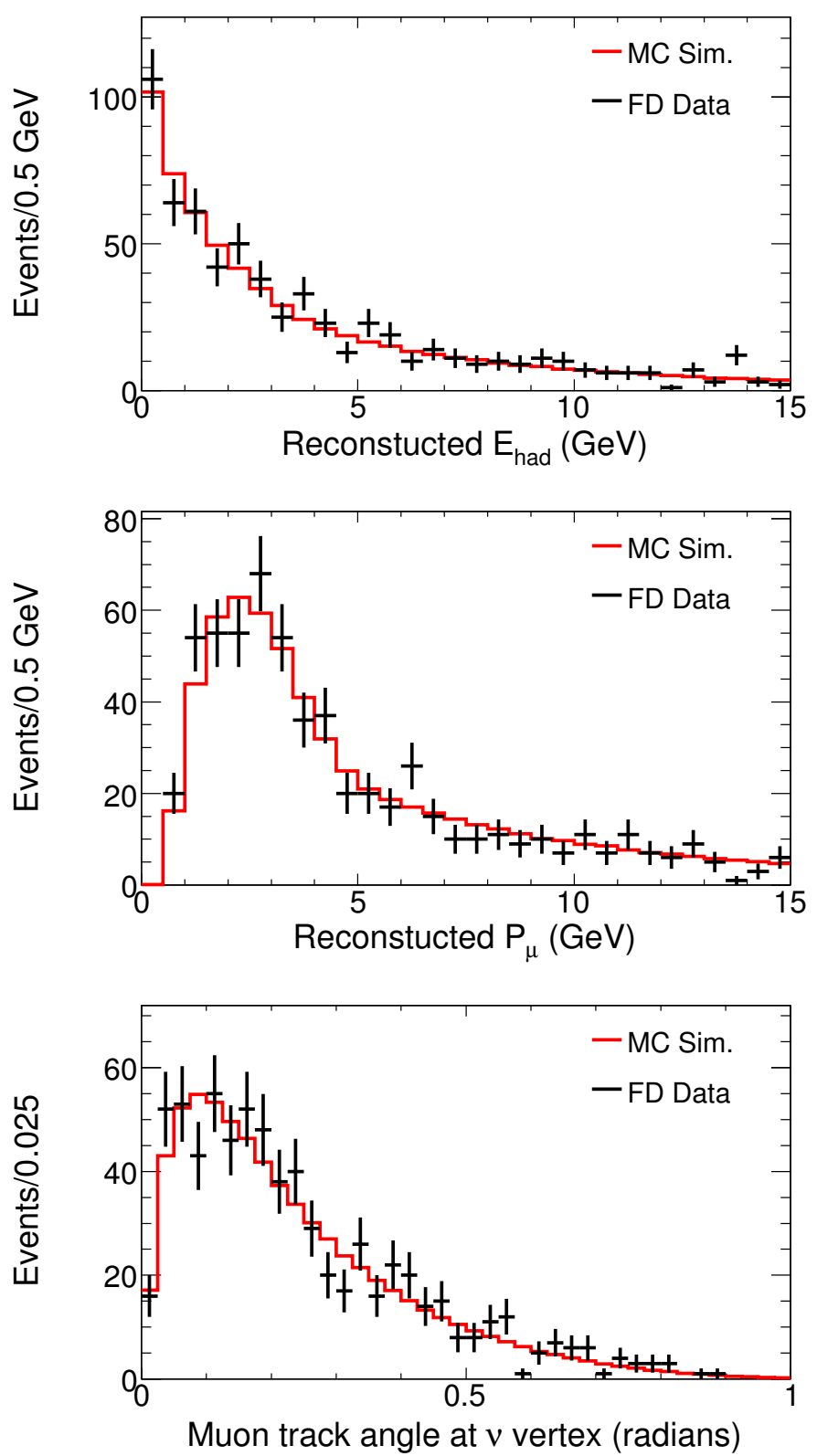

Figure 3.5: Reconstructed shower energy (top), muon momentum (middle), and muon angle (bottom) for the selected $\nu_{\mu}$ charged-current events for the far detector data and $\mathrm{MC}$ simulation. The event selection procedure is described in Chapter 8. The MC simulation includes flux and cross-section corrections computed in Chapter 7 and corrections for the neutrino oscillations computed in Chapter 9. 

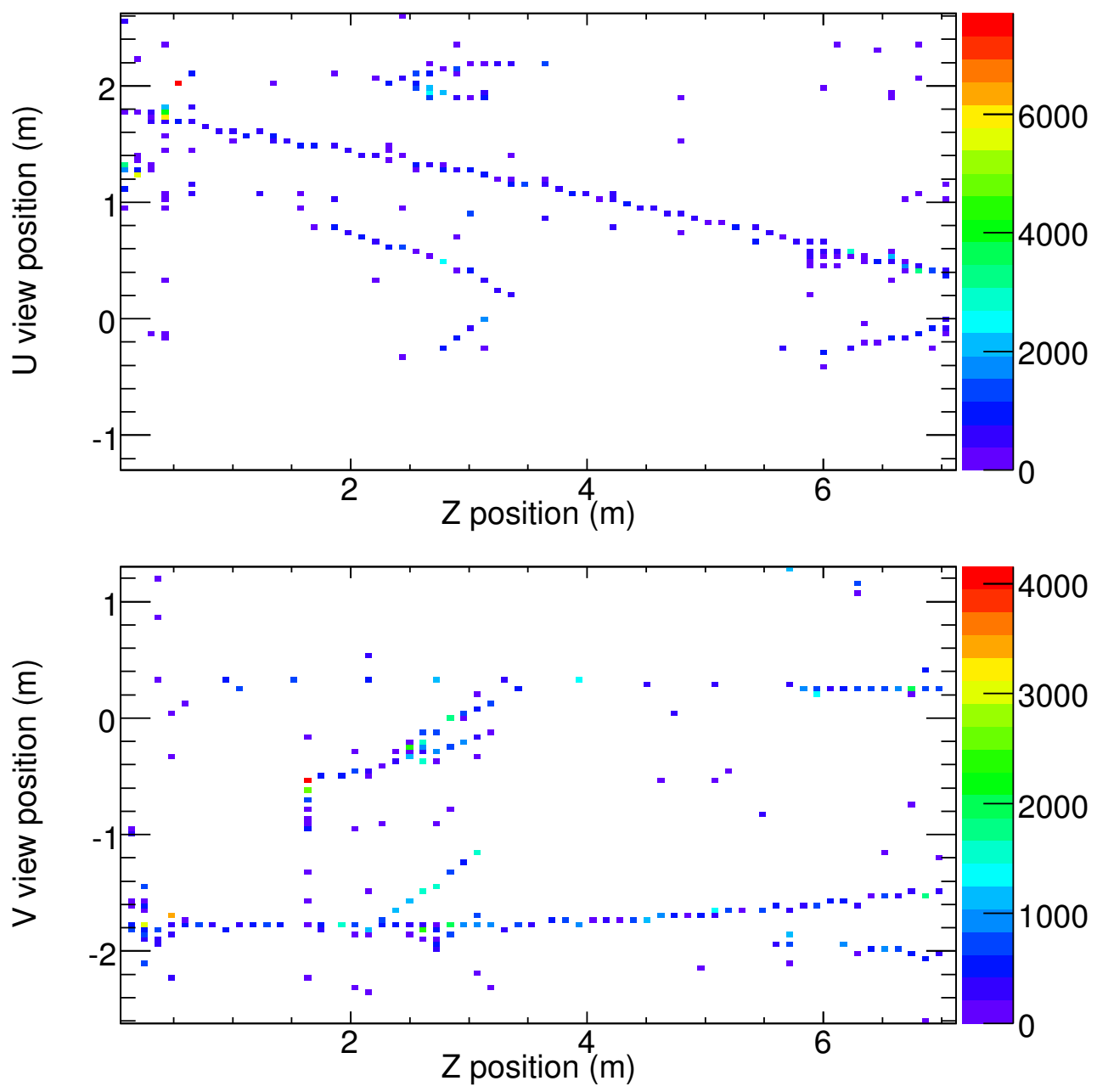

(a)

Figure 3.6: This figure shows one neutrino beam spill in the near detector. All hits with pulse height greater than 40 ADC from the calorimeter section of the near detector are shown. Neutrinos are incident from the left. This spill contains multiple neutrino interactions within the detector and one rock muon entering through the front face of the detector. The hit pattern and hit times are used to reconstruct individual events. The hit pulse height is measured using ADC, where $100 \mathrm{ADC} \approx 1$ photoelectron. 


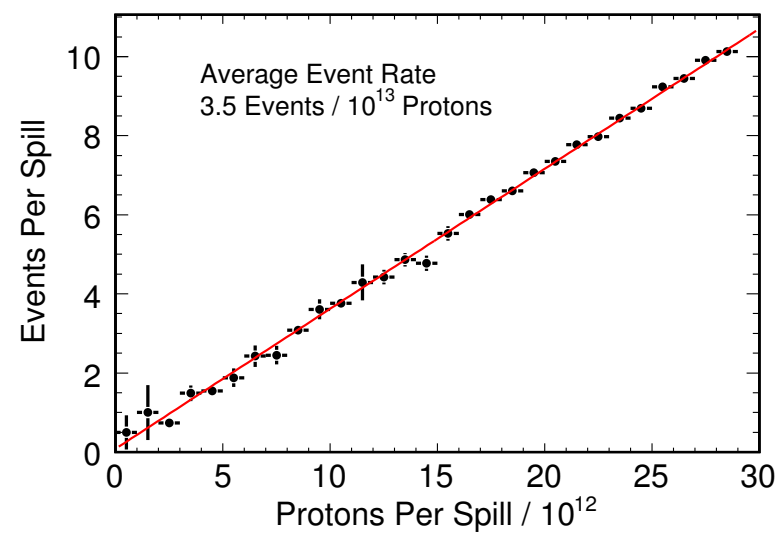

Figure 3.7: A mean number of near detector events per near detector beam spill plotted as a function of the number of protons on target (POT). The plot was taken from [58].

and deep inelastic scattering (DIS). The total $\nu_{\mu}$ charged-current cross-section and these contributions are shown in Figure 3.8. Examples of simulated QES, RES, and DIS events are shown in Figures 3.9, 3.10, and 3.11.

QES interactions produce muons and protons. Figure 3.9 shows a typical reconstructed QES event. In this example, no visible energy from the final state proton is observed in the detector. The absence of the visible hadronic energy is an efficient signature of the QES events in the MINOS detectors [80].

RES interactions produce muons and baryon resonances. Figure 3.10 shows a typical reconstructed RES event in which the baryon resonance $\Delta(1232)$ has decayed into a proton and pion. The resonance production process is dominated by a single pion production; this process proceeds through 3 channels [81]: $\nu_{\mu} p \rightarrow \mu^{-} p \pi^{+}, \nu_{\mu} n \rightarrow \mu^{-} p \pi^{0}$, and $\nu_{\mu} n \rightarrow \mu^{-} n \pi^{+}$. The resonance production also includes contributions from higher mass resonances. As illus- 


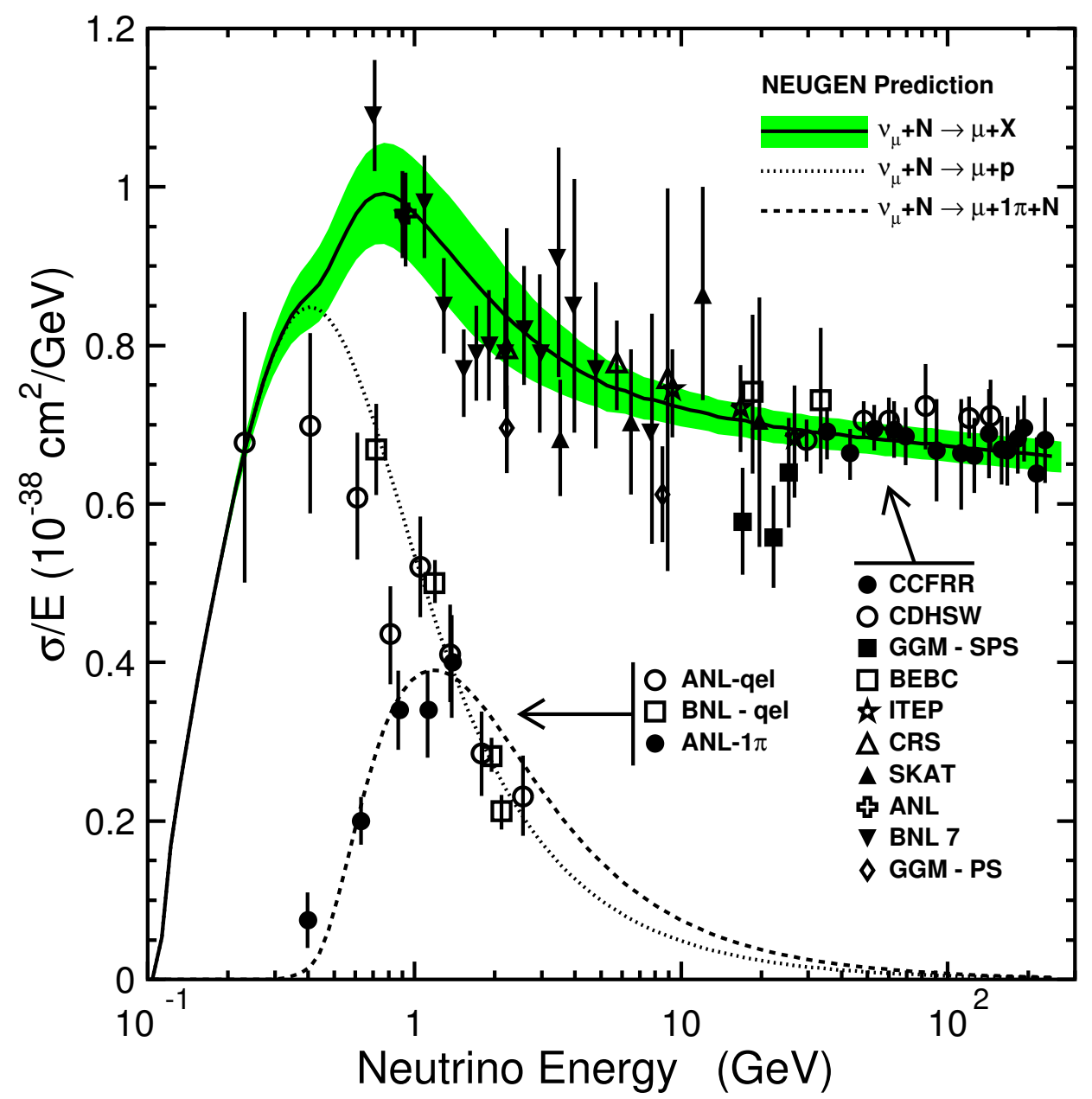

Figure 3.8: Total cross-section for $\nu_{\mu}$ charged-current interactions per nucleon on an isoscalar target, as predicted by the NEUGEN event generator [78]. The cross-sections per $\mathrm{GeV}$ are shown as a function of the neutrino energy for inclusive scattering $\left(\nu_{\mu}+N \rightarrow \mu+X\right)$, quasielastic scattering $\left(\nu_{\mu}+N \rightarrow \mu+p\right)$, and single pion production $\left(\nu_{\mu}+N \rightarrow \mu+\pi+N\right)$. The calculation is compared with the experimental data tabulated in [79]. The shaded band corresponds to the cross-section uncertainties. This figure was taken from [58]. 

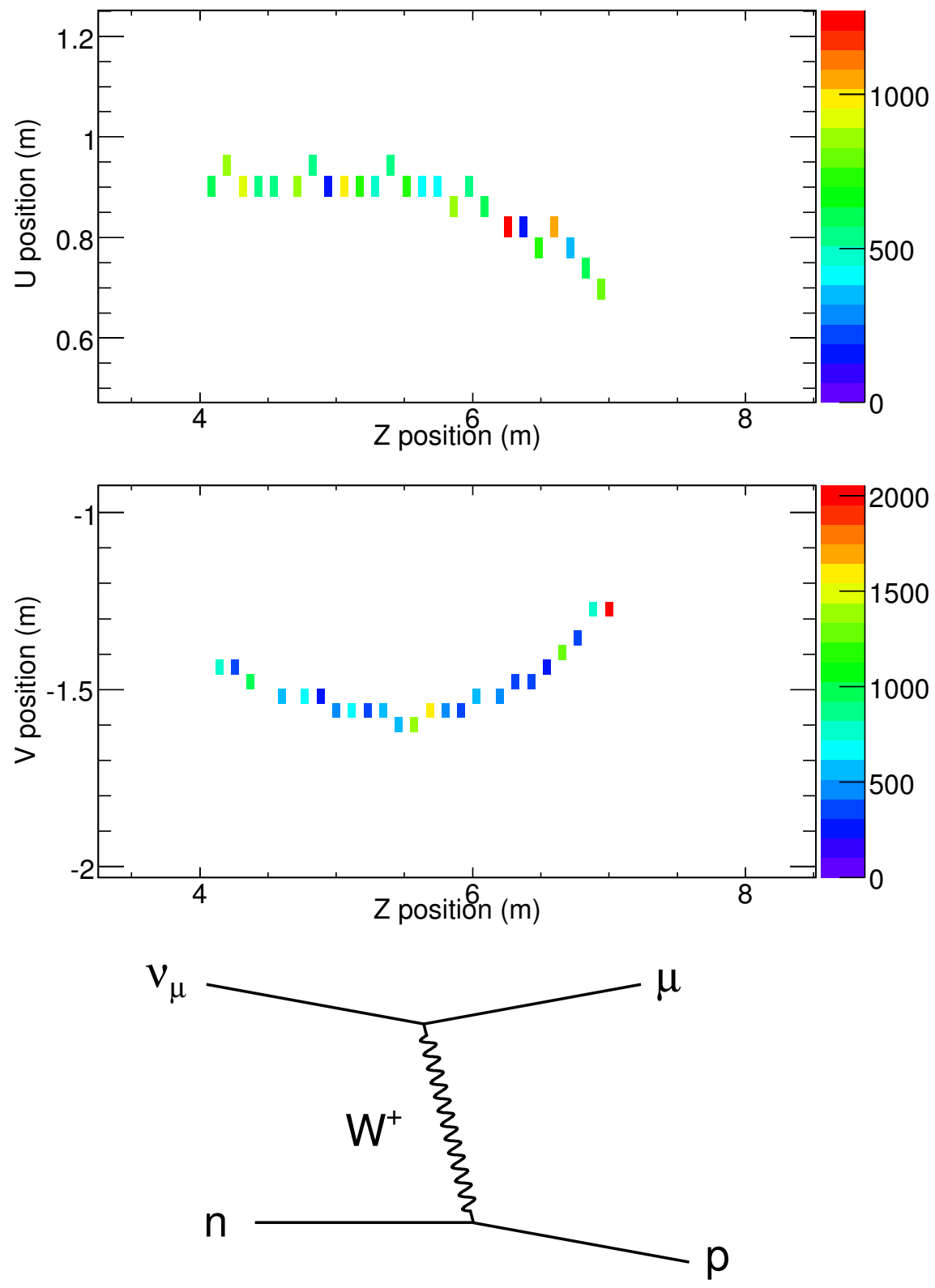

Figure 3.9: Event display and Feynman diagram for the quasielastic scattering (QES) $\nu_{\mu}$ charged-current interaction. The top two panels show the reconstructed QES event in the MC simulation of the near detector. 

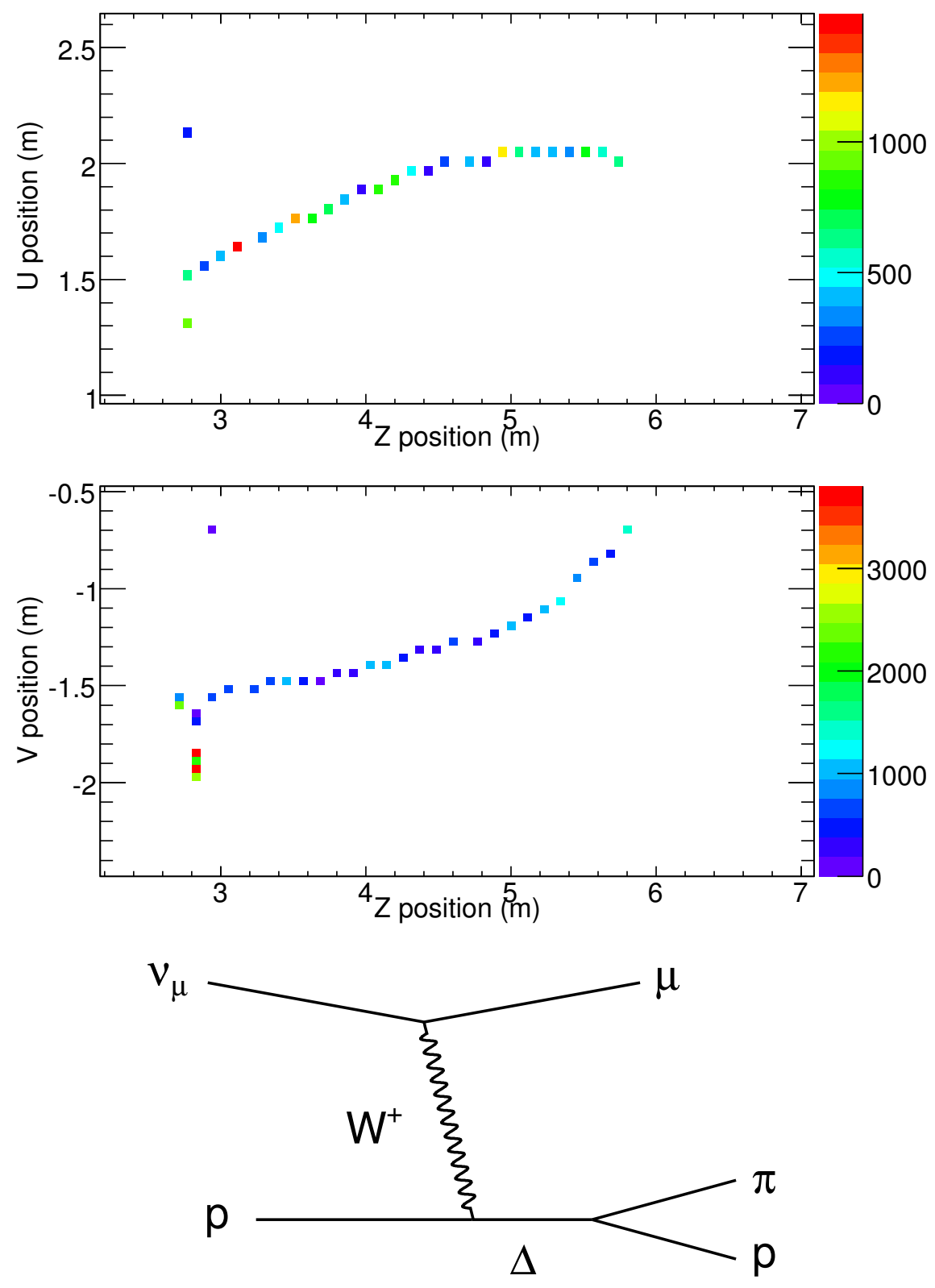

Figure 3.10: Event display and Feynman diagram for the resonance production (RES) $\nu_{\mu}$ charged-current interaction. The top two panels show the reconstructed RES event in the MC simulation of the near detector. 

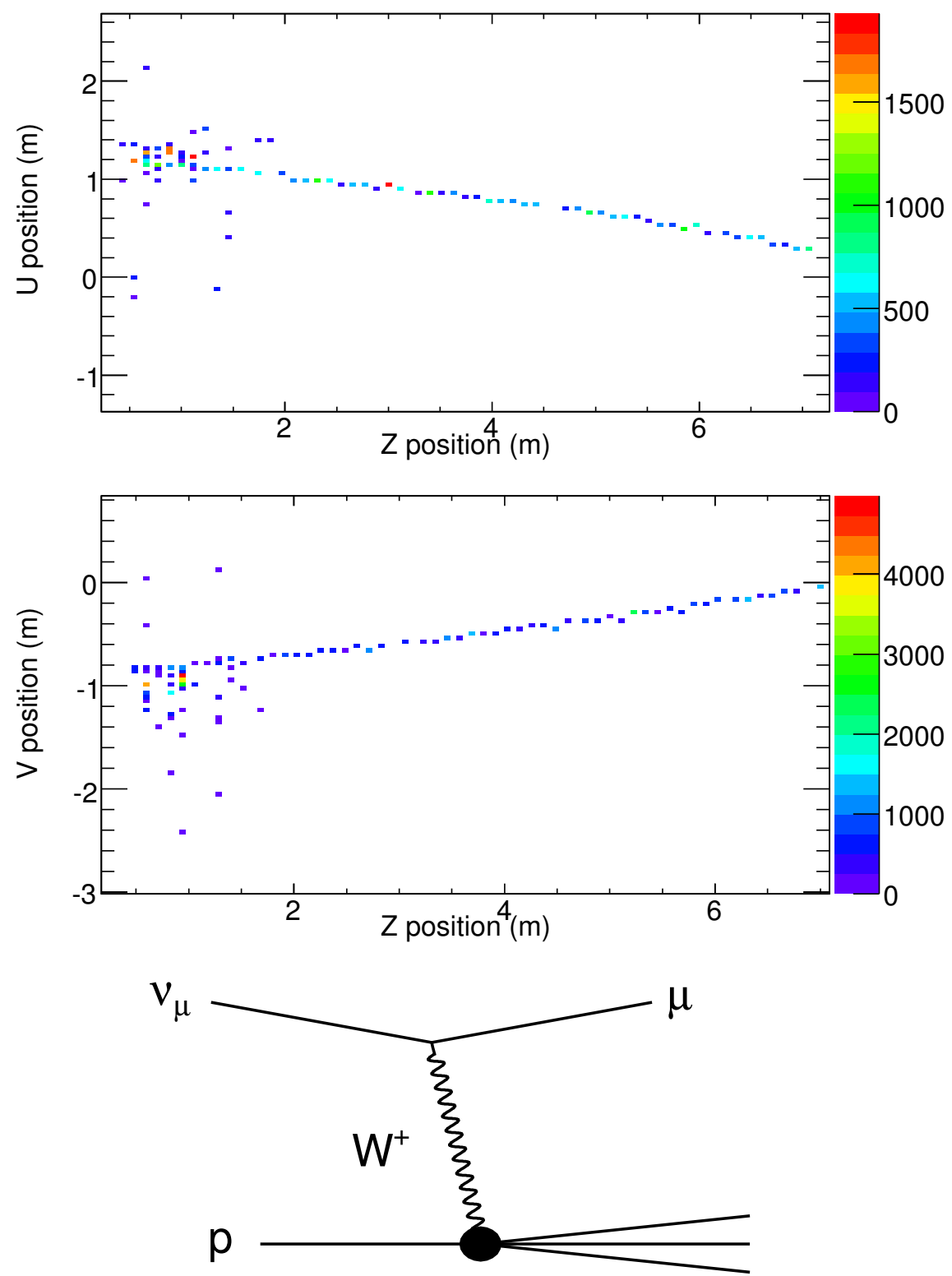

Figure 3.11: Event display and Feynman diagram for the deep inelastic scattering (DIS) $\nu_{\mu}$ charged-current interaction. The filled circle represents the internal proton structure which is described by the parton and gluon structure functions. The top two panels show the reconstructed DIS event in the MC simulation of the near detector. 
trated by this example, the MINOS detectors can not resolve tracks due to the pions and protons.

DIS interactions produce muons and hadronic cascades. Figure 3.11 shows a typical reconstructed DIS event. The event display clearly shows a hadronic shower with multiple detector hits.

Hadrons are produced by neutrino interactions within a nucleus; interactions of hadrons with a nucleus are called "final state interactions" (see Section 3.5). These interactions can change the multiplicity and the particle type of the hadrons produced in neutrino interactions. For example, if a pion from a RES interaction is absorbed by a nucleus, then a detector can only observe a single proton. This RES interaction becomes indistinguishable from a QES interaction. These nuclear interactions occur before a particle can be observed, and they introduce irreducible systematic effects for a measurement of QES and RES cross-sections on heavy nuclei. Understanding these nuclear effects presents experimental and theoretical challenges for low energy neutrino experiments. $^{3}$

\subsection{Selection of QES, RES, and DIS events}

In this section, a method to select QES, RES, and DIS events is developed, using events from the Monte-Carlo simulation. This work is motivated by two goals. First, a measurement of oscillation parameters requires an ac-

\footnotetext{
${ }^{3}$ see Reference [81] for a review.
} 
curate measurement of hadronic shower energy in the MINOS detectors. The QES, RES, and DIS events produce distinct hadronic cascades; thus, a study of these events is a step toward improving our understanding of hadronic showers. The MINOS near detector accumulated millions of $\nu_{\mu}$ charged-current events, which allowed for a detailed study of QES, RES, and DIS interactions using high statistics event samples. Second, the energy from the QES and RES interactions is measured more precisely than energy from the DIS interactions, as discussed in Section 3.4.

Table 3.1 lists fractions of the true QES, RES, and DIS events among the selected $\nu_{\mu}$ charged-current events in the MC simulation of the low energy beam configuration. In the MC simulation, each reconstructed event is matched to the true neutrino interaction that generated the detector hits for this reconstructed event.

Figures 3.12 and 3.13 show the reconstructed variables, including reconstructed energy, for the true QES, RES, and DIS events. The DIS events dominate at high neutrino energies, and the QES and RES events dominate at low neutrino energies. In the MINOS experiment, a muon neutrino disappearance at the far detector is maximal around $1.5 \mathrm{GeV}$ (see Figure 1.2). The QES and RES events can be used to measure precisely the shape of the neutrino energy spectrum, as discussed in Chapter 9 .

A visual scan of the QES, RES, and DIS events was performed using the MC simulation of the near detector. No clear features were found that would have allowed for the identification of the QES, RES, and DIS events. This 


\begin{tabular}{l|r} 
Type & Fraction (\%) \\
\hline QES & $17.6 \%$ \\
RES & $25.6 \%$ \\
DIS & $56.8 \%$
\end{tabular}

Table 3.1: Fractions of the true QES, RES, and DIS events among the selected $\nu_{\mu}$ charged-current events, computed with the MC simulation of neutrino interactions in the near detector using the low energy beam configuration.

MC study agrees with the test beam data accumulated with the calibration detector (see Section 2.4). The protons and pions, with momenta around $1 \mathrm{GeV}$, were observed to produce only a few detector hits [72]. These hits do not provide enough information to identify the proton or pion tracks in the MINOS detectors. As a result, individual QES and RES events cannot be identified in the MINOS detectors. Instead, a procedure was developed to select events similar to QES, RES, and DIS events. The three categories of events are defined using reconstructed variables; each category is enriched with either QES, RES, or DIS events.

Figure 3.12 shows reconstructed muon momenta, shower energy, and muon track angles for the true QES, RES, and DIS events. Approximately $40 \%$ of the QES events have no reconstructed hadronic energy. These QES events can be selected by requiring $E_{\text {had }}<150 \mathrm{MeV}$.

It is useful to introduce invariant quantities to describe the neutrino 

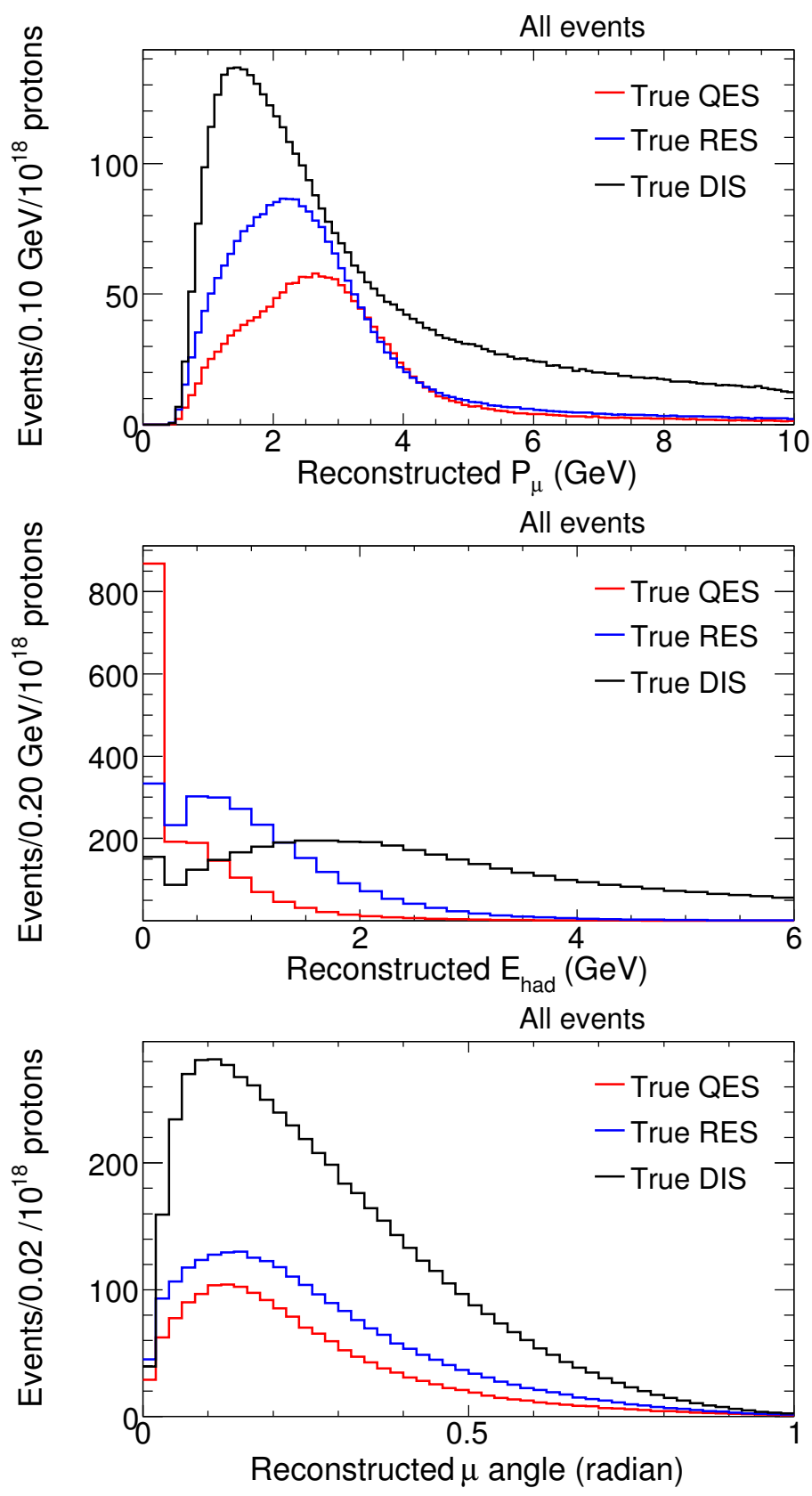

Figure 3.12: Reconstructed quantities for the true QES, RES, and DIS events among the selected MC $\nu_{\mu}$ charged-current events. The top figure shows the reconstructed muon momentum. The middle figure shows the reconstructed shower energy. The bottom figure shows the reconstructed muon track angle. 


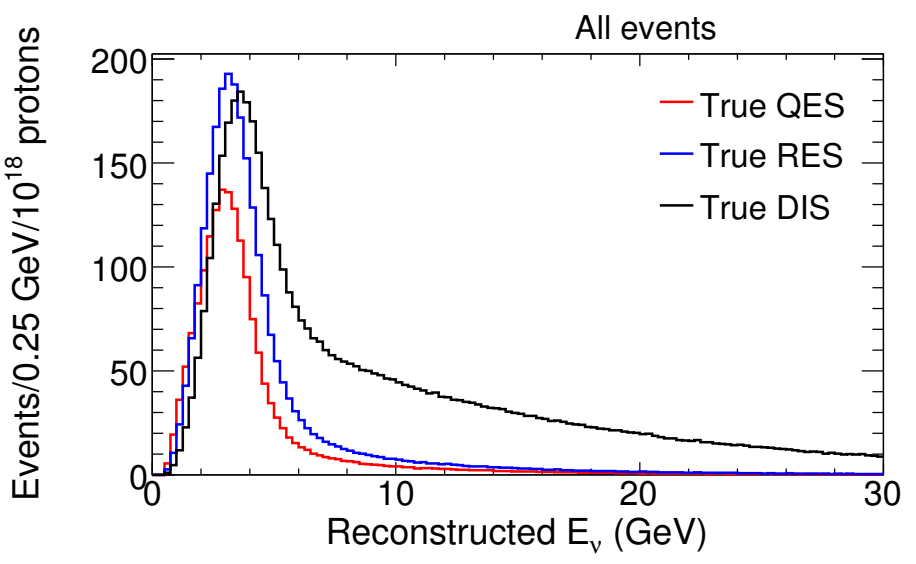

Figure 3.13: Reconstructed event energy for the true QES, RES, and DIS events among the selected $\mathrm{MC} \nu_{\mu}$ charged-current events.

interactions:

$$
\begin{aligned}
y & =\frac{p \cdot q}{p \cdot k_{1}} \text { (inelasticity), } \\
Q^{2} & =-q^{2} \text { (negative squared four-momentum), } \\
X & =\frac{Q^{2}}{2 p \cdot q}(\text { Bjorken scaling variable }), \\
W^{2} & =(p+q)^{2} \text { (squared invariant mass of final state), }
\end{aligned}
$$

where the four-momentum vectors $p, q$, and $k_{1}$ are defined in Figure 3.14. These quantities are commonly used for the analysis of data in neutrino experiments [82].

A laboratory coordinate system is defined to express the above invariant quantities as a function of the three detector observables: shower energy $E_{\text {had }}$, muon momentum $P_{\mu}$, and muon angle $\theta_{\mu}$. The $\mathrm{Z}$ axis of the laboratory coordinate system is aligned along the direction of the NuMI neutrino beam. The target nucleon is assumed to be at rest with a mass $M$. In the laboratory 


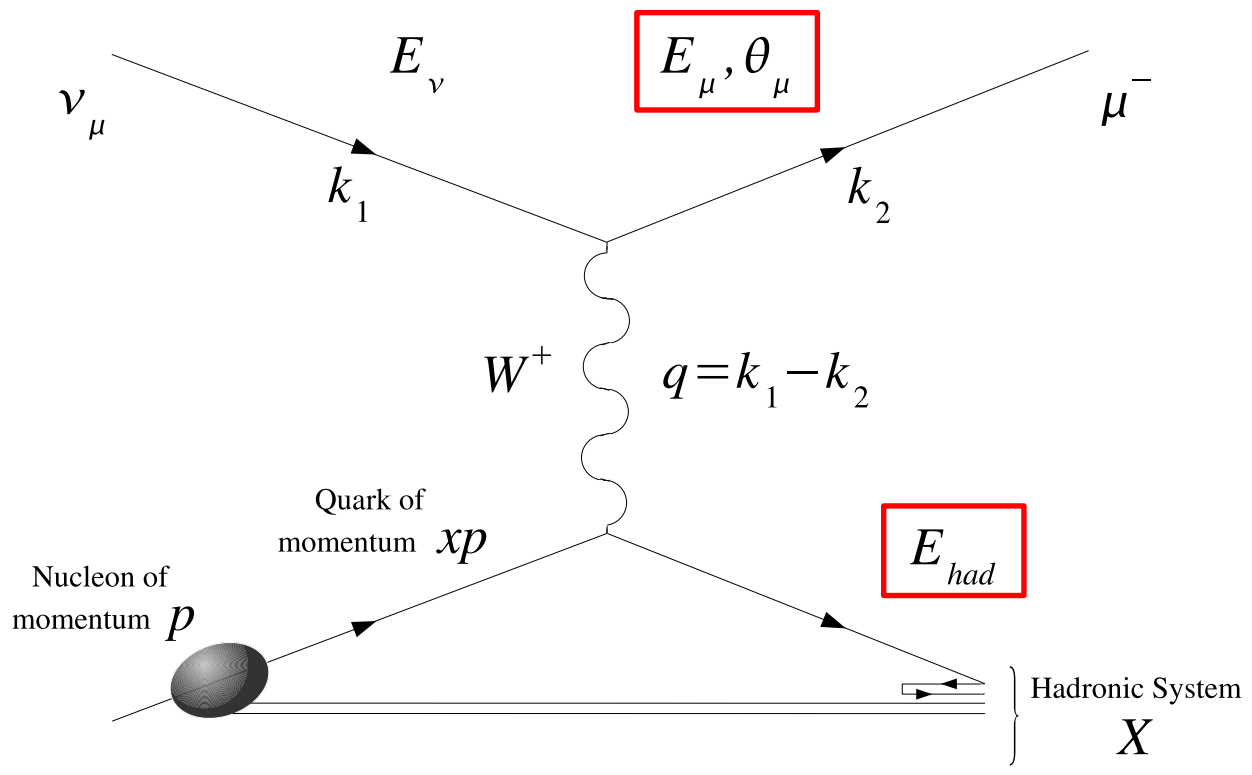

Figure 3.14: Feynman diagram of $\nu_{\mu}$ charged-current interaction with nucleon. Three detector observables are measured for every reconstructed event: energy of final-state hadronic system (hadronic shower energy) $E_{\text {had }}$, muon energy $E_{\mu}$, and muon angle $\theta_{\mu}$ with respect to the initial neutrino direction. Here, $k_{1}$ is the four-momentum of incoming neutrino, $p$ is the four-momentum of target nucleon, $k_{2}$ is the four-momentum of outgoing muon, and $q$ is the fourmomentum of $W^{+}$boson. The figure was taken from [80]. 
frame, the above expressions reduce to:

$$
\begin{aligned}
y & =\frac{E_{\text {had }}}{E_{\nu}}, \\
Q^{2} & =E_{\nu} E_{\mu}\left(1-\cos \theta_{\mu}\right), \\
X & =\frac{Q^{2}}{2 M E_{h a d}}, \\
W^{2} & =M^{2}+2 M E_{h a d}+Q^{2} .
\end{aligned}
$$

The negative squared four-momentum $Q^{2}$ and squared invariant mass of final state $W^{2}$ are shown in Figure 3.15. This figure shows two categories of events: all events and events with $E_{\text {had }}>150 \mathrm{MeV}$. Among the events with $E_{\text {had }}>150 \mathrm{MeV}$, the majority of the RES events have values of $W^{2}$ less than $4 \mathrm{GeV}^{2}$. This observation is consistent with the $\Delta$ resonance mass $m_{\Delta}=1.233 \mathrm{GeV}$ and the proton mass $m_{p}=0.938 \mathrm{GeV}[5]$.

Based on the above observations, a simple method is created to separate the QES, RES, and DIS events:

QES selection: $E_{\text {had }}<150 \mathrm{MeV}$,

RES selection: $E_{\text {had }}>150 \mathrm{MeV}$ and $W^{2}<4 \mathrm{GeV}^{2}$,

DIS selection: $E_{\text {had }}>150 \mathrm{MeV}$ and $W^{2}>4 \mathrm{GeV}^{2}$.

The MC energy spectra for the selected QES, RES, and DIS events are shown in Figure 3.16. These categories of events will be used for the analysis of the near detector data in Chapter 7 and the analysis of the far detector data in Chapter 9. 

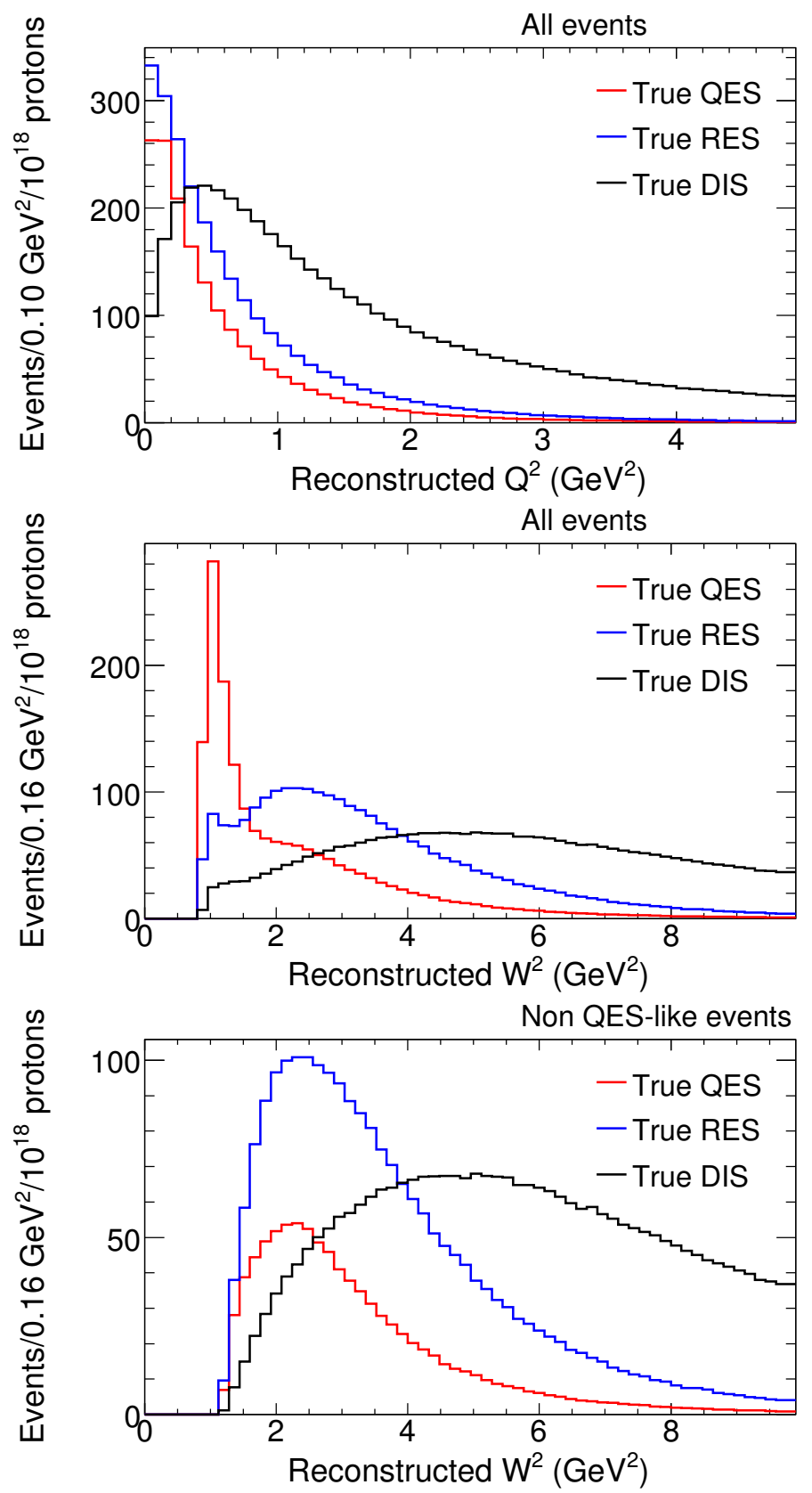

Figure 3.15: Reconstructed $W^{2}$ and $Q^{2}$ variables for the true QES, RES, and DIS events. The top and middle figures show all selected MC $\nu_{\mu}$ chargedcurrent events. The bottom figure shows the events with $E_{\text {had }}>150 \mathrm{MeV}$. 

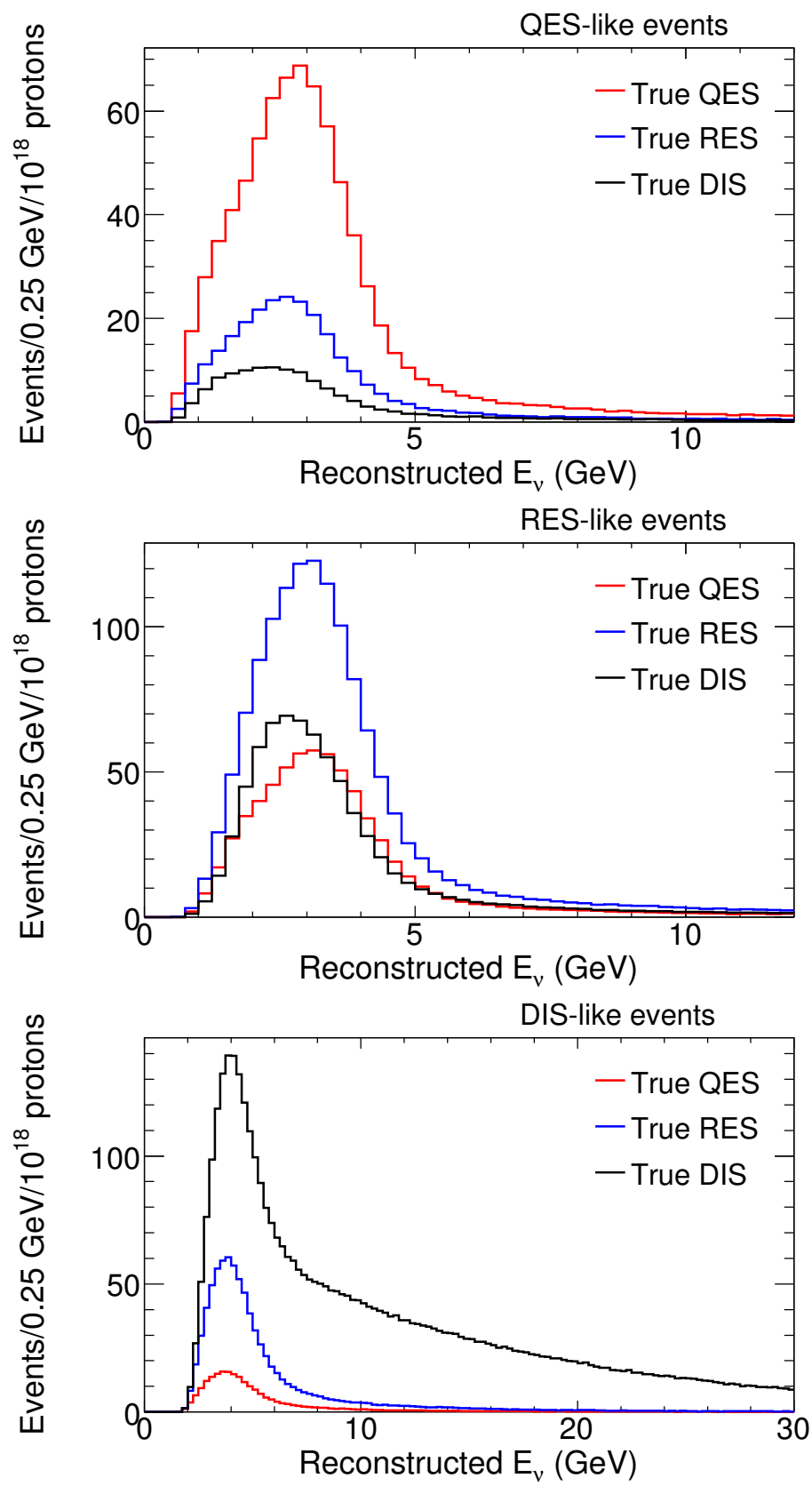

Figure 3.16: Reconstructed event energy for 3 categories of the selected MC $\nu_{\mu}$ charged-current events. The top figure shows the selected QES events. The middle figure shows the selected RES events. The bottom figure shows the selected DIS events. In each figure, the contributions from the true QES, RES, and DIS events are shown separately. 

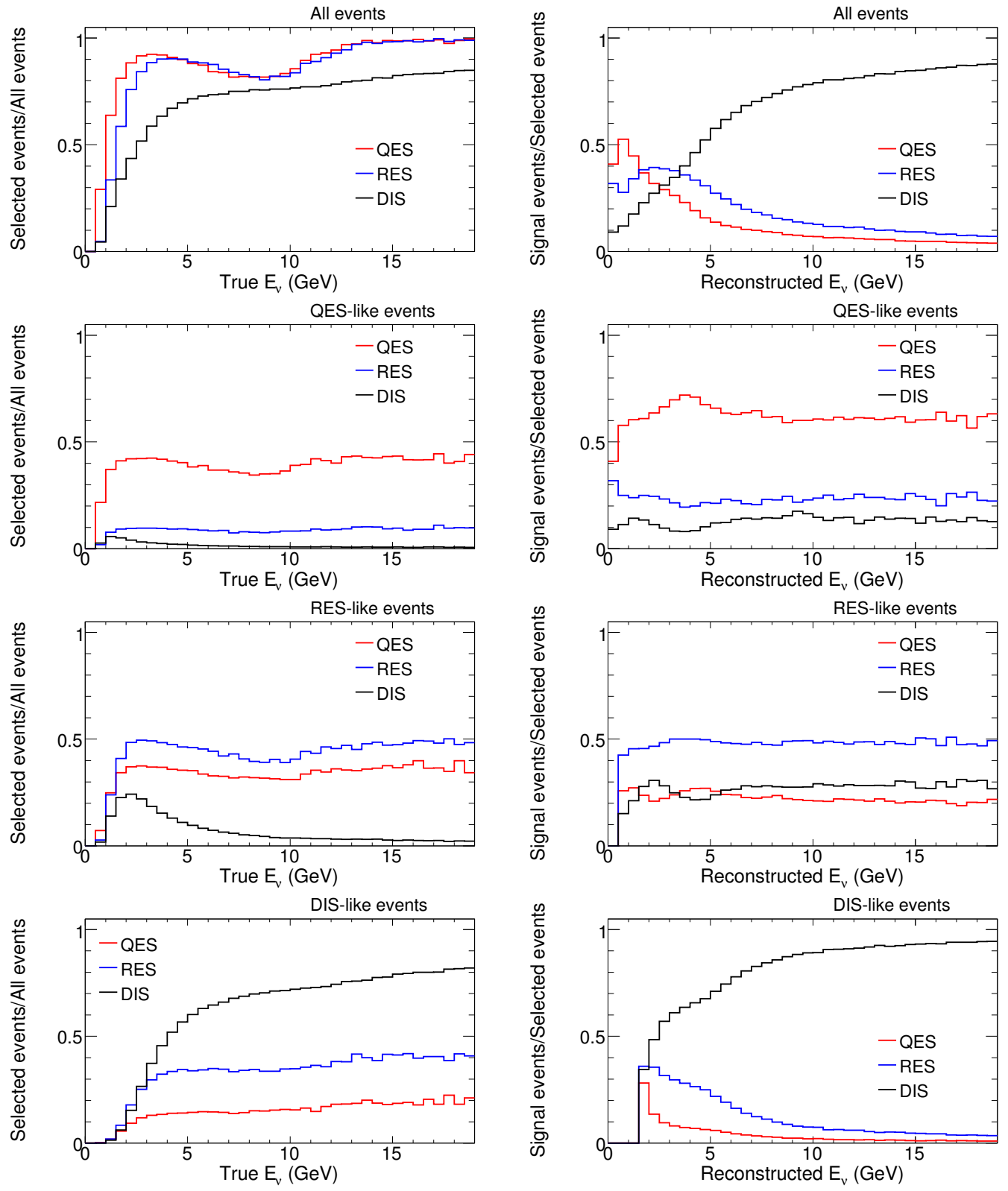

Figure 3.17: Purity and efficiency (defined in the text) for true QES, RES, and DIS components for the selected categories of the QES, RES, and DIS events and for all selected events. 
Figure 3.17 shows purity and efficiency for the selected QES, RES, and DIS categories. The efficiency is the number of selected signal events divided by the total number of signal events (before a selection). The purity is the number of background events among the selected events divided by the total number of selected events. For example, in the QES category, the true QES events are signal events. In each category of events, the required event type is a majority of selected events.

\subsection{Energy reconstruction in QES, RES, and DIS events}

In this section, the reconstructed event energy is examined for QES, RES, and DIS events, using events from the Monte-Carlo simulation. For this Monte-Carlo study, it is useful to define these energy resolution quantities:

$$
\begin{aligned}
\sigma_{\mu} & =\frac{E_{\mu}^{\text {reco }}-E_{\mu}^{\text {true }}}{E_{\mu}^{\text {true }}}, \\
\sigma_{\text {had }} & =\frac{E_{\text {had }}^{\text {reco }}-E_{\text {had }}^{\text {true }}}{E_{\text {had }}^{\text {true }}}, \\
\sigma_{\nu} & =\frac{E_{\nu}^{\text {reco }}-E_{\nu}^{\text {true }}}{E_{\nu}^{\text {true }}},
\end{aligned}
$$

where $E_{\mu}^{r e c o}$ is the reconstructed muon momentum, $E_{\text {had }}^{r e c o}$ is the reconstructed hadronic shower energy, and $E_{\nu}^{r e c o}$ is the reconstructed event energy $\left(E_{\nu}^{r e c o}=\right.$ $\left.E_{\mu}^{\text {reco }}+E_{\text {had }}^{\text {reco }}\right) . E_{\mu}^{\text {true }}$ is the true muon momentum, $E_{\text {had }}^{\text {true }}$ is the true hadronic shower energy, and $E_{\nu}^{\text {true }}$ is the true neutrino energy. The true values are generated by the MC simulation. The energy resolution estimates the accuracy of the energy reconstruction, taking into account detector response effects. 
Figure 3.18 shows the energy resolution computed for the true QES, RES, and DIS events (left figures). For the true QES and RES events, the energy reconstruction consistently underestimates the amount of visible hadronic energy, as shown in Figure 3.18a. This bias is likely due to statistical fluctuations in the detector response to a single hadron. Figure 3.18e shows the shower energy resolution for the true DIS events; these events have a nearly unbiased energy reconstruction.

Figure 3.18 also shows the energy resolution computed for the selected QES, RES, and DIS categories (right figures). The energy reconstruction for the selected RES and DIS events performs reasonably well, as shown in Figure 3.18b. However, the QES requirement selects the reconstructed events with no visible hadronic energy. This requirement results in a significant underestimate of the reconstructed shower energy for the selected QES event category, as shown in Figure 3.18f.

The energy reconstruction for the selected QES events can be improved by including the muon angle. If the nuclear effects are ignored, the QES interaction is a two-body scattering process; this is illustrated in Figure 3.9. The momentum of an incoming neutrino is aligned along the neutrino beam direction, and it is assumed that the target nucleon is at the rest. The hadronic shower energy, muon momentum, and muon angle are measured by the detector. Using the energy and momentum conservation, the event energy for the 


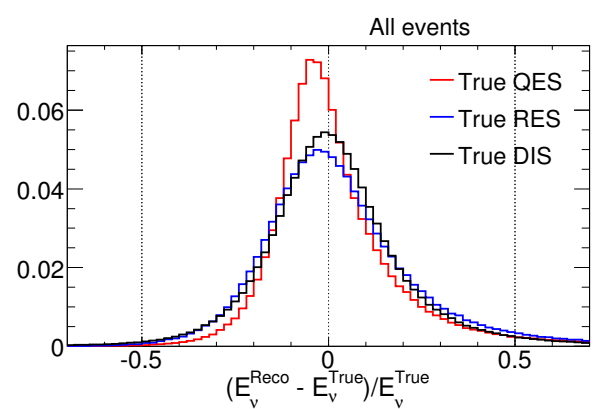

(a)

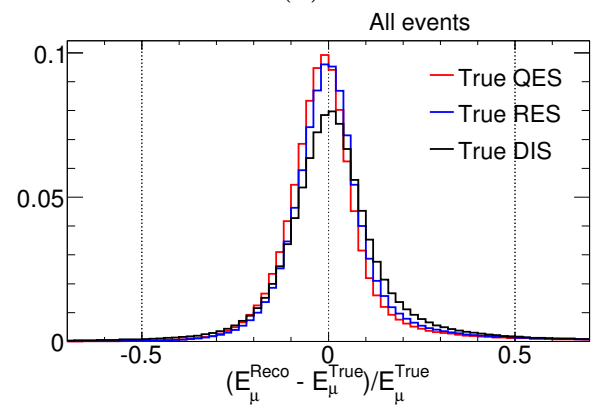

(c)

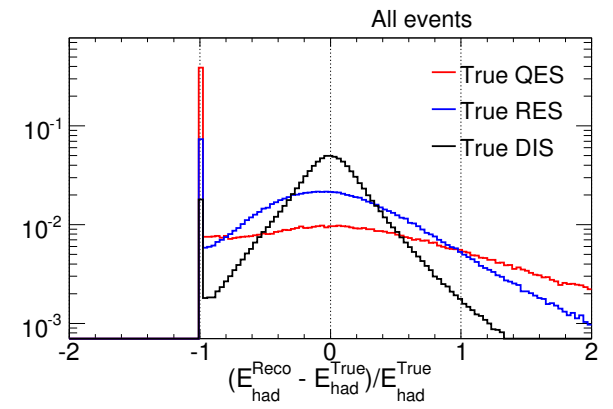

(e)

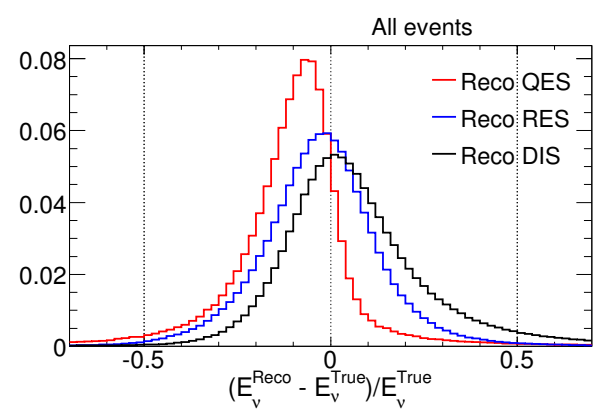

(b)

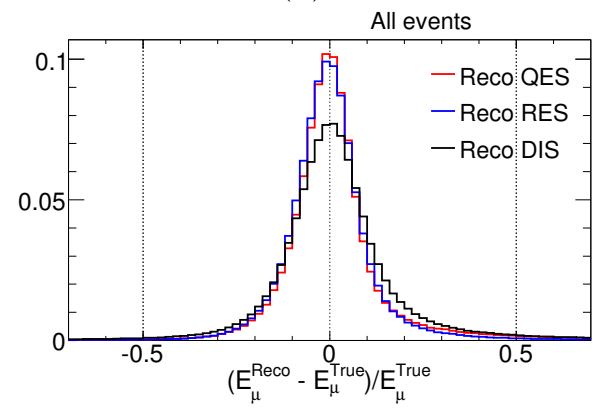

(d)

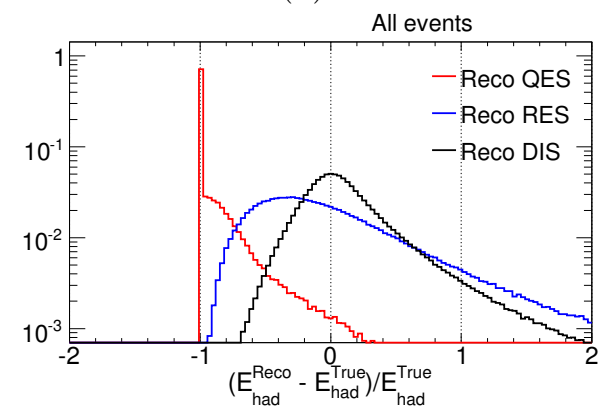

(f)

Figure 3.18: Energy resolution for $E_{\nu}$ (top), $E_{\mu}$ (middle), and $E_{\text {had }}$ (bottom). Figures (a), (c), and (e) show the energy resolution computed for the true QES, RES, and DIS events in the MC simulation. Figures (b), (d), and (f) show the energy resolution computed for the selected QES, RES, and DIS events, as discussed in the text. The selected QES, RES, and DIS categories include the contributions from the true QES, RES, and DIS events, as illustrated in Figure 3.16. 


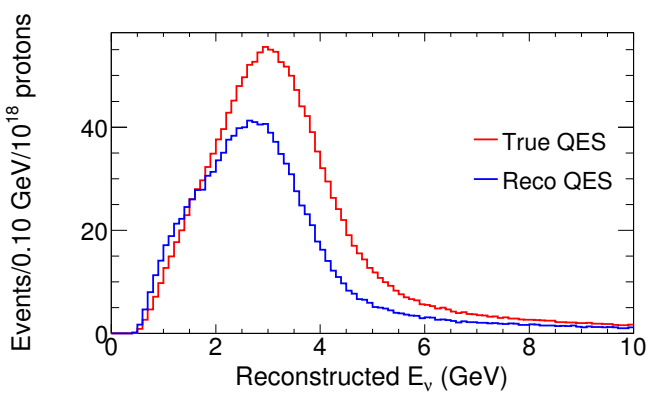

(a)

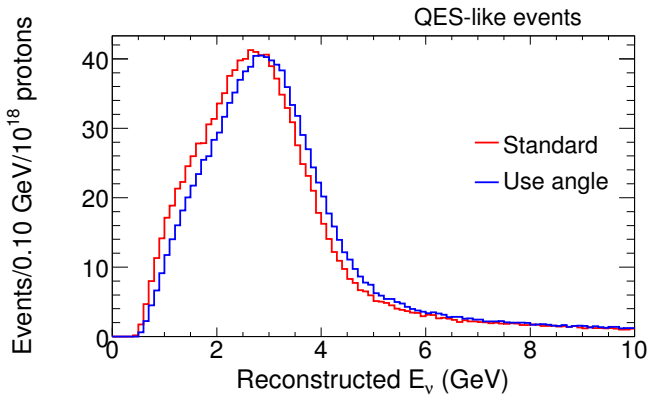

(c)

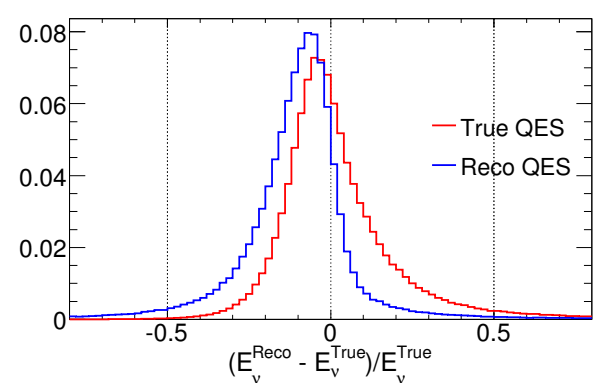

(b)

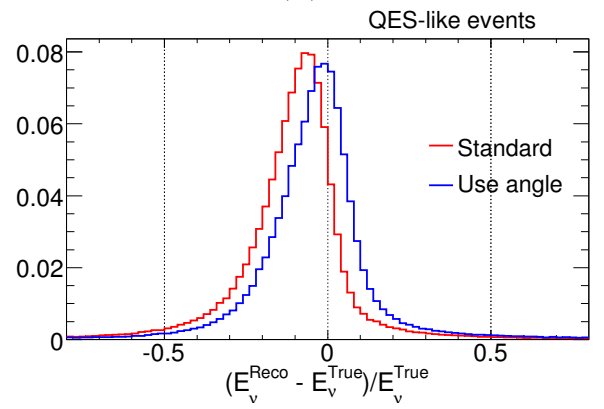

(d)

Figure 3.19: Figure (a) shows the reconstructed energy for the true and selected (reco) QES events. Figure (b) shows the energy resolution for the true and selected (reco) QES events. The bottom two plots compare the two energy reconstruction methods, defined in Equation 3.1 (red) and Equation 3.5 (blue). Figure (c) shows the reconstructed energy for the selected QES events. Figure (d) shows the energy resolution for the selected QES events. Using Equation 3.5 significantly reduces the energy reconstruction bias. 
QES events can be expressed as a function of the three detector observables:

$$
E_{Q E S}=\frac{\left(M_{p}-E_{b i n}\right) E_{\mu}+\frac{1}{2}\left(M_{p}^{2}-\left(M_{p}-E_{b i n}\right)^{2}-m_{\mu}^{2}\right)}{M_{p}-E_{b i n}-E_{\mu}+P_{\mu} \cos \theta_{\mu}},
$$

where $M_{p}$ is the proton mass, $E_{b i n}$ is the nucleon binding energy ${ }^{4}$, and $m_{\mu}$ is the muon mass.

The standard method estimates the neutrino energy as the sum of hadronic shower energy and muon energy. The standard method and the method defined in Equation 3.5 are compared in Figure 3.19. Figure 3.19a shows the reconstructed event energy for the true QES events and the events selected as the QES events. Figures 3.19b shows the energy resolution for the true QES events and the events selected as the QES events. These figures show that a part of the neutrino energy ${ }^{5}$ can be recovered by including the reconstructed muon angle. A similar study for the RES events concluded that Formula 3.5 does not improve the energy reconstruction for the RES events.

\subsection{Monte-Carlo simulation}

The MC simulation of the MINOS experiment is used to develop analysis methods and to compute a predicted event rate the far detector. This MC simulation contains three components:

- Simulation of the proton interactions with the graphite target, propagation of the secondary pions and kaons, and generation of the neutrino

\footnotetext{
${ }^{4}$ The iron binding energy is approximately $36 \mathrm{MeV}$.

${ }^{5}$ This part is carried by the undetected proton.
} 
beam from the pion and kaon decays within the decay pipe.

- Simulation of neutrino interactions with a detector, including the simulation of cross-sections and kinematic distributions of the secondary hadrons and leptons and the interactions of final state hadrons with a nucleus.

- Propagation of the secondary particles through the detector material and magnetic field and the simulation of the interactions of these particles with the active detector components.

The first (beam simulation) MC component is outlined in Chapter 2. The neutrino interactions in the MINOS detectors are modeled using the NEUGEN neutrino event generator [78]. NEUGEN simulates neutrino interactions in the $100 \mathrm{Mev}-100 \mathrm{GeV}$ range for the charged-current interactions of the muon, electron, and tau neutrinos and neutral-current interactions. The generator includes a cross-section library that contains the theoretical expectations and experimental data for the total and differential neutrino crosssections, summarized in Figure 3.8. The cross-sections are used to compute a probability for a neutrino interaction within a detector or rock material around the detector. NEUGEN produces a list of particles generated in the neutrino interaction; these particles are passed on to the detector simulation.

The MINOS detectors are constructed from steel (95\% of the detector mass), plastic scitillator (5\% of the detector mass), and a small amount of aluminimum. The majority of the neutrino interactions occur in steel. The 
target nucleons are bound within nuclei; in NEUGEN the bound nucleons are described by the Fermi Gas model [83]. The target nucleons are off-shell and have a Bodek-Ritchie momentum distribution [84], with a cutoff set at $500 \mathrm{MeV}$ within the NEUGEN simulation. The initial neutrino-nucleon interaction is simulated in the nucleon rest frame, where kinematic variables for the final state leptons and hadrons are selected based on the free nucleon differential cross-sections. For the QES interactions the final nucleon energy has to be greater than the Fermi momentum, $p_{F}=236 \mathrm{MeV}$ for iron; otherwise, the interaction is rejected.

Final state leptons are allowed to leave the target nucleus without additional interactions and are passed directly to the detector simulation. Hadronic final states can interact with the nucleus (final state interactions), which changes the momentum and the multiplicity of the final state hadrons. The final state interactions calculations in NEUGEN use INTRANUKE, which incorporates pion elastic and inelastic scattering, single charge exchange, and absorption $[78,85]$. The final state interactions reduce the amount of visible hadronic energy in the detector. This is the largest systematic error for the measurement of the hadronic shower energy in the MINOS detectors $[86,87]$.

The MINOS detector simulation randomly draws neutrinos from the MC neutrino flux and traces them through the detectors and detector halls. The neutrinos are allowed to interact within the detector, surrounding hall structures and rock material. The secondary particles produced in these interactions are traced through the realistic model of the MINOS detectors, 
including the effects of the magnetic field. These interactions of the secondary particles with the MINOS detectors are simulated using GEANT3 [62]. The energy depositions within the active detector elements (scintillator strips) are passed to the MC simulation of the detector response. This step in the simulation uses a simulation model developed by MINOS.

The response of individual channels to identical energy deposition can vary from channel to channel. This is due to variations in the gains of the photomultiplier tubes, the quality of the scintillator, and the quality and the length of the optical fiber (see Section 2.3). The MINOS experiment employs calibration procedures to measure the response of the individual detector channels, as discussed in Section 2.4. The MC simulation of the detector readout system accurately represents the non-uniform detector response. It also reproduces the actual characteristics of the scintillator strips, the photomultiplier tubes, and the optical fibers in the real detector. The fully calibrated response of the real and the simulated detectors to a muon passage through a scintillator strip agree at the $2 \%$ level $[52,70]$. Chapter 4 will shows that the remaining differences between the data and $\mathrm{MC}$ simulation do not affect the events selected for our analysis.

The overall response of the near detector was independently checked with the muons produced in the interactions of the beam neutrinos. A charged particle passing through a scintillator strip generates a detector hit, as discussed in Section 2.3. The pulse height of the hit is a function of energy loss in the scintillator strip. The amount of energy deposited by a muon in a scin- 
tillator strip approximately follows the Landau distribution [88]. The signal produced by the muon is smeared by the detector response. The scintillator and wave-length shifting fiber response, photoelectron statistics, and fiber attenuation are the most significant detector smearing effects. The recorded energy scintillator strip signal for the muons is a convolution of the Landau function and detector response function, which can be approximated by the Gaussian function. The shape of the detector response function is in reasonable agreement with the MC simulation [89].

\subsection{Study of near detector hits in the data and MC simulation}

This section compares a number of neutrino induced hits in the near detector data and the MC simulation. A mean number of all detector hits in a spill is a function of the number of neutrino interactions and mean neutrino energy. The MC simulation includes realistic neutrino flux and neutrino interaction models, as discussed in Section 3.5. The MC simulation also includes an accurate model of the near detector response. A number of detector hits in the data and $\mathrm{MC}$ simulation are counted to verify that the MC simulation accurately reproduces the near detector response. This study is independent from the reconstruction algorithms because all near detector hits are counted. Figure 3.6 shows an example of all the near detector hits produced in one neutrino beam spill.

This study uses neutrino beam spills accumulated with the target placed 


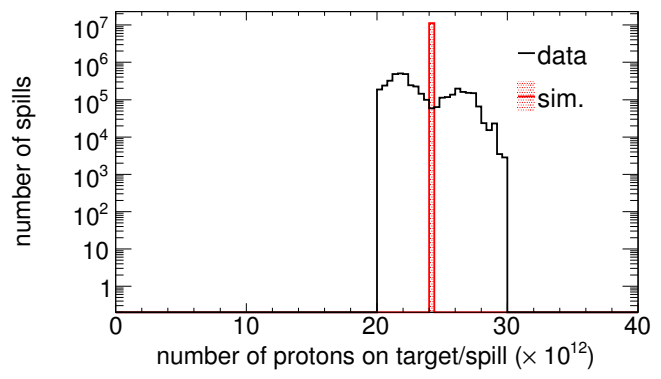

Figure 3.20: A number of protons delivered to (simulated with) the NuMI target for the data (MC) spills.

in the low beam configuration. Table 3.2 summarizes a number of spills for the data and the MC simulation. On average, the MC spills contain the same number of protons on target (POT) as the data spills. The number of POT per spill is shown in Figure 3.20. In this section, the MC distributions are normalized to the number of POT in the data.

\begin{tabular}{l|r|r|r} 
& Spills & Number of protons & Mean number of protons/spill \\
\hline Data & 4218091 & $0.9777 \times 10^{20}$ & $23.18 \times 10^{12}$ \\
\hline Simulation & 11263201 & $2.7257 \times 10^{20}$ & $24.20 \times 10^{12}$
\end{tabular}

Table 3.2: Summary of the data (MC) spills recorded (simulated) with the target placed in the low energy target position.

A detector hit signal (pulse height) is measured using a partially calibrated scintillator strip response. The response of each strip is calibrated to match the average detector response (see Section 2.3). The signal is measured using ADC units. where $100 \mathrm{ADC} \approx 1$ photoelectron. Any detector hit with a signal greater than $40 \mathrm{ADC}$ is counted. Hits within a $120 \mathrm{~ns}$ window are combined into a single hit, if these hits are separated by a span less than 60 ns. 
Figure 3.21 shows the number of detector channels (scintillator strips) with one or more hits in the same channel for the data and MC simulation. On average, the data spills contain a greater number of the active channels than predicted by the MC simulation. The data spills also contain significantly more channels with multiple hits than the MC simulation. Understanding the sources of these additional hits is a subject of ongoing work by the MINOS collaboration. A working hypothesis is that these hits are caused by a delayed light emission within a photomultiplier tube after the tube is exposed to light from the earlier physical hit [90]. This effect was first observed in 2005 when it was noticed that the near detector channels for a few percent of the hits record an additional hit following the earlier hit in the same channel. This effect is attributed to a delayed light emission within a photomultiplier tube, and it is not included in the MC simulation. The reconstruction algorithms were modified [91] to suppress activity in a channel for $2 \mu$ s after the earlier hit in the channel.

Figure 3.22a shows the number of all the detector hits, and Figure 3.22c shows the mean signal of all the detector hits. These two figures do not include hits from the channels that have 8 or more hits in a single spill; the hits from these channels are shown in Figures $3.22 \mathrm{~b}$ and $3.22 \mathrm{~d}$. The shapes of the distributions (comparing the left and right panels) in Figure 3.22 are very different. The channels with multiple hits are likely due to faulty ("bad") electronics channels. ${ }^{6}$ Typically, there were a few faulty channels present in

\footnotetext{
${ }^{6}$ This observation is based on our experience with monitoring the near detector during
} 

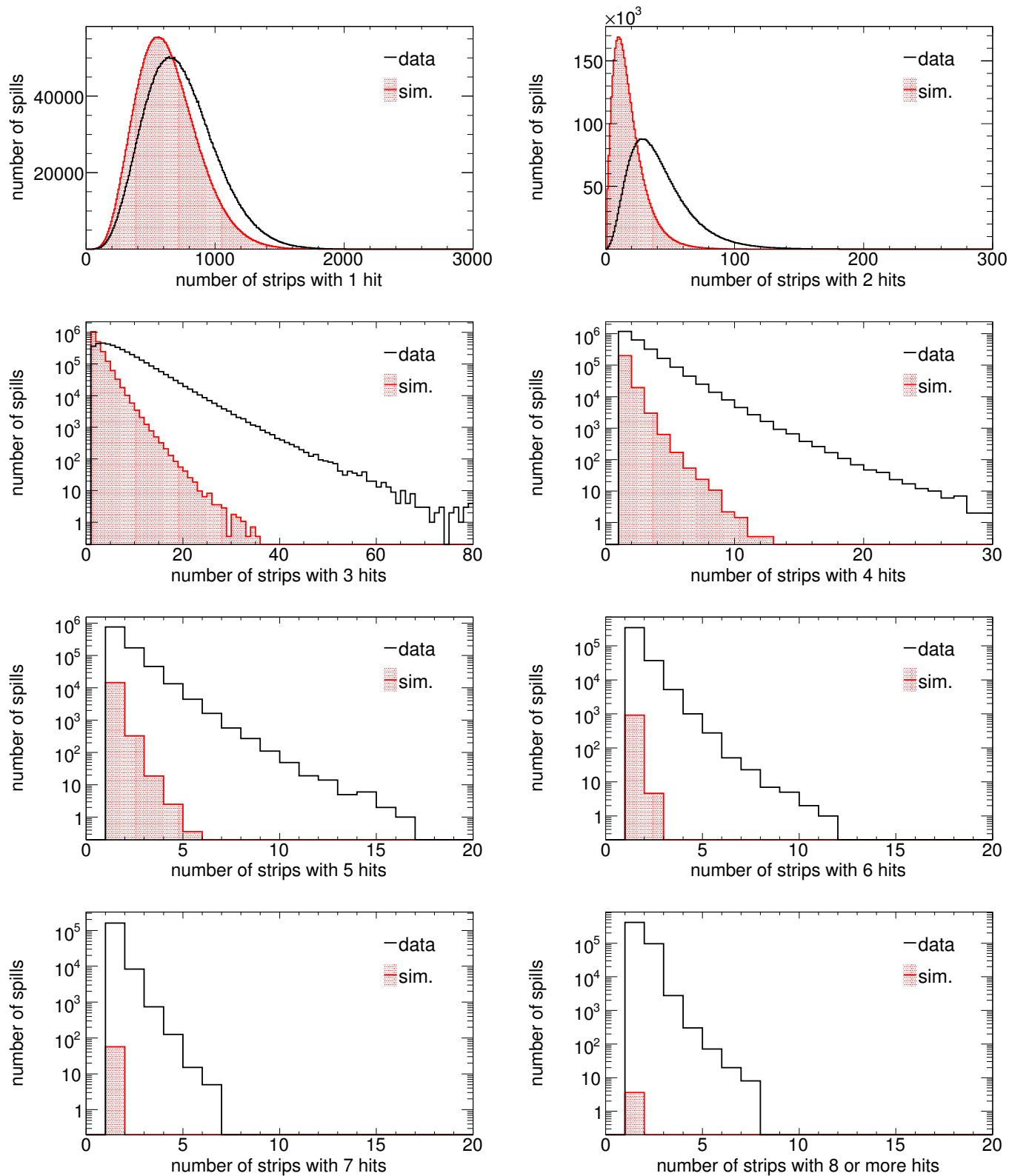

Figure 3.21: Response of the near detector to neutrino beam spills. The figures show the number of channels in a spill that have 1, 2, 3, 4, 5, 6, 7, or 8 or more hits within a single spill. The multiple hits in the same channel are separated in time. 
the near detector during any period before August of 2008. At that time, the near detector electronics boards were modified to reduce substantially the occurrence of faulty channels. A majority of the faulty channels are excluded from the analysis using the error code generated by the near detector electronics. Figure 3.22 shows the remaining "bad" channels without the error codes. In this section, any detector channel with 8 or more hits within a single spill is also excluded from the analysis. A fraction of the "bad" channels was always less than $0.1 \%$ of all near detector channels, and they did not affect analysis of the near detector data.

The physical and "noise" hits can be separated by applying a signal (pulse height) threshold. Hits with a signal below the threshold are called "low signal hits." Hits with a signal above the threshold are called "high signal hits." Next, the low and high signal strips are compared in the data and MC simulation.

A minimum ionizing particle (MIP) response of the near detector scintillator strips is approximately 6 photoelectrons or 600 ADC. As a result, the mean pulse height of the physical detector hits is a few photoelectrons. A mean number of the physical hits per spill is a function of the neutrino flux and cross-section. Figure 3.23 shows the number of the detector hits below and above the four signal thresholds: 1.2, 2, 2.8, and 3.6 photoelectrons. The number of high signal hits agrees with the MC simulation. The difference be-

control room shifts. 


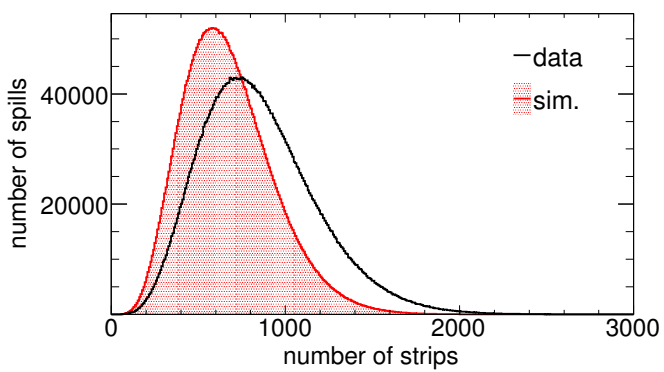

(a)

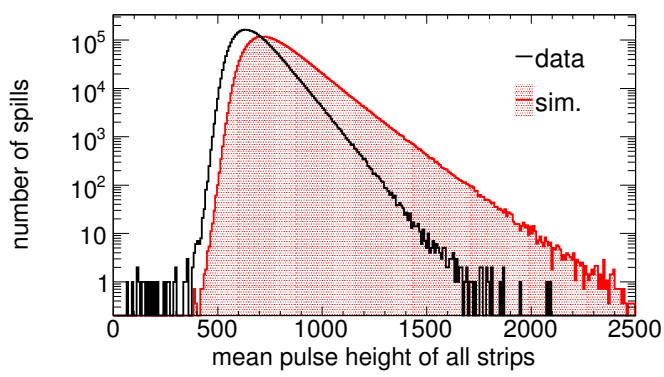

(c)

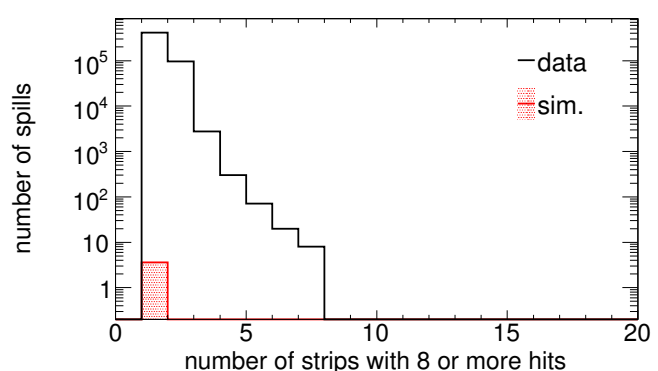

(b)

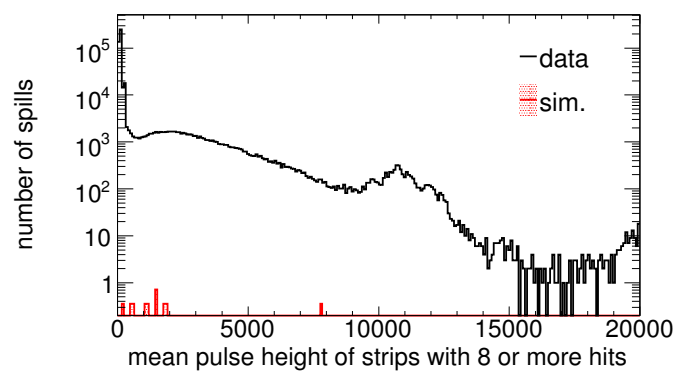

(d)

Figure 3.22: Response of the near detector to neutrino beam spills. Figure (a) shows the number of all active channels. Figure (b) shows the number of channels with 8 or more hits in a single spill. Figure (c) shows the mean signal of all active channels. Figure (d) shows the mean signal of channels with 8 or more hits in a single spill. The MC histograms are normalized to the number of POT in the data. 

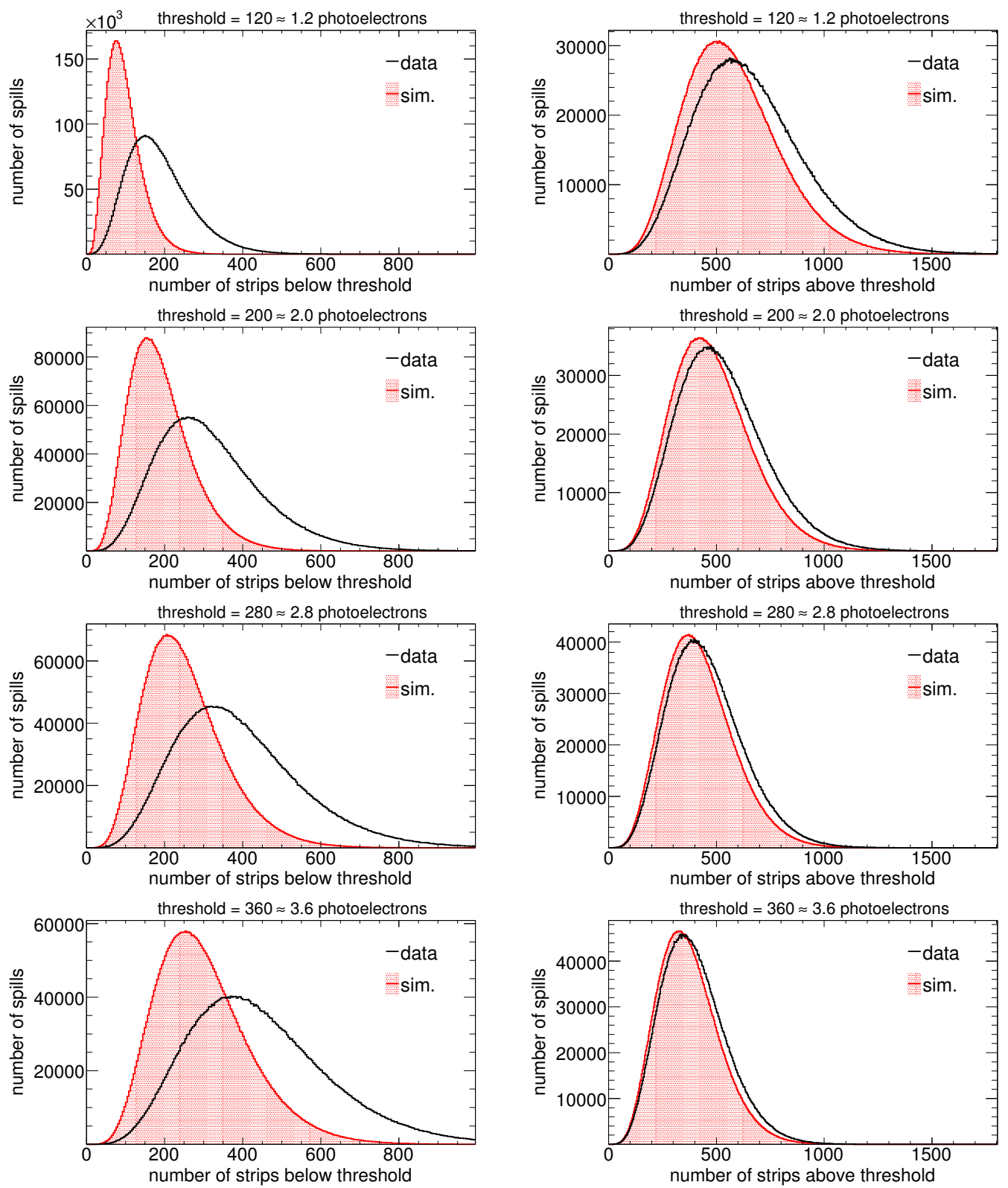

Figure 3.23: Response of the near detector to neutrino beam spills. The figures show the number of detector hits below (left) and above (right) the signal threshold, as labeled on each plot. The X axis labels use "strips" to stand for hits. 

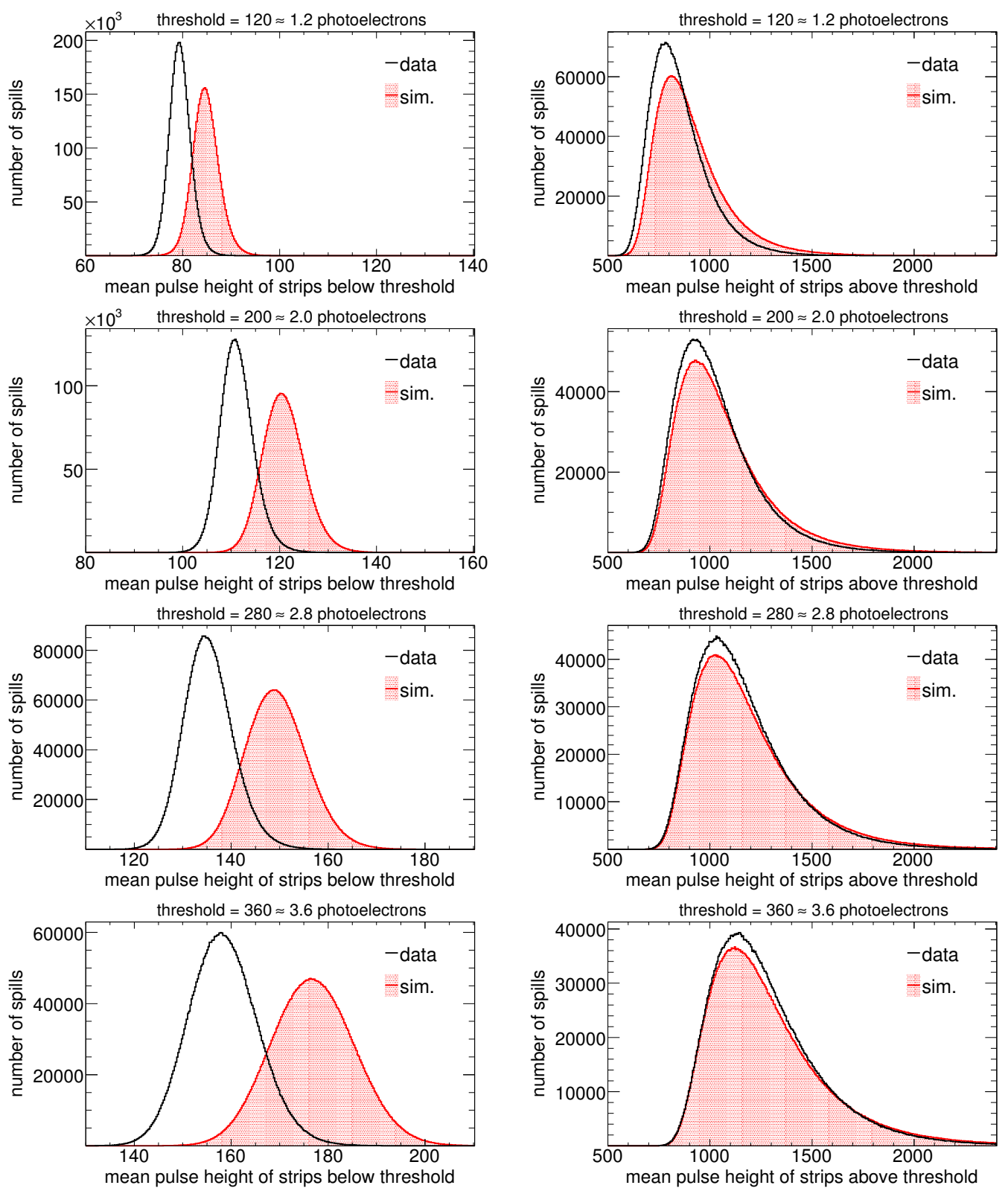

Figure 3.24: Response of the near detector to neutrino beam spills. The figures show the mean signal of detector hits with the signal below (left) and above (right) the signal threshold, as labeled on each plot. The $\mathrm{X}$ axis labels use "strips" to stand for hits. 
tween the data and the MC simulation is around a few percent, and it can be explained by uncertainties in the MC models of the neutrino flux and crosssections. In Chapter 7, flux and cross-section MC models are tuned using the near detector data.

The number of detector hits in the data with a signal less than 2 photoelectrons (low signal hits) is significantly greater than the number of these hits in the MC simulation. The mean number of the low signal hits depends on a number of optical cross-talk hits. A typical cross-talk hit is around 1 photoelectron. Properties of the photomultiplier tubes, including optical cross-talk, were measured using a test stand [92,93]; these measurements were included in the MC simulation. Figure 3.23 shows that the data spills contain additional low signal hits that are not accurately modeled by the MC simulation. Preliminary observations [90] suggest that many of these additional hits follow a physical hit within a few $\mu$ s window.

Figure 3.24 shows the mean signal of the low and high signal detector hits for the four threshold values. The mean signal of the high signal hits in the data agrees with the MC simulation. This observation implies that the physical hits in the data are accurately modeled by the $\mathrm{MC}$ simulation.

The response of the near detector and far detector readout systems was studied using the calibration detector, as discussed in Chapter 2. The response of the readout systems to physical hits is reproduced by the MC simulation at a better than a $1 \%$ level [65]. In Chapter 4, it will be shown that the additional data hits with a signal less than 2 photoelectrons do not affect $\nu_{\mu}$ charged- 
current events in the near detector. It is concluded that $\nu_{\mu}$ charged-current events in the far detector are also not affected by the low signal hits.

\subsection{Summary}

This chapter opened with the description of the event reconstruction and followed with the discussion of the $\nu_{\mu}$ charged-current interactions in the MINOS detectors. The $\nu_{\mu}$ charged-current cross-sections for the quasielastic scattering (QES), resonance production (RES), and deep inelastic scattering (DIS) processes overlap in the $1 \mathrm{GeV}$ to $5 \mathrm{GeV}$ region. The absolute QES and RES cross-sections are constrained by the previous neutrino experiments, but the normalization uncertainties are greater than the statistical precision of the MINOS near detector data. In this section, a simple method was developed to select QES, RES, and DIS events. These events will be used in Chapter 7 to tune the normalization of the QES and RES cross-sections, using the MINOS near detector data.

In addition, a measurement of the QES and RES event normalizations in the near detector allows the computation of the interaction rates of these events at the far detector. As a result, the oscillation analysis of the far detector data can be performed independently for the QES, RES, and DIS events. The energy of the QES events can be reconstructed with a higher resolution possibly improving the precision of the muon neutrino disappearance measurement. This study is discussed in Chapter 9.

This chapter also briefly discussed the Monte-Carlo (MC) simulation 
of the MINOS experiment. The MC simulation is used to develop analysis techniques and to compute a prediction for the $\nu_{\mu}$ charged-current event rate at the far detector without oscillations. However, the near detector data contains a substantial number of low pulse hits, which are not modeled by the MC simulation. It will be shown in Chapter 4 that these low pulse height hits do not introduce a systematic error to the analysis of $\nu_{\mu}$ charged-current events. 


\section{Chapter 4}

\section{Identification of muon tracks}

The MINOS experiment measures the muon neutrino disappearance rate by comparing the $\nu_{\mu}$ charged-current event rates at the near and far detectors. These events are identified by the observation of a muon track. Low energy muons produce short tracks, which are difficult to distinguish from background tracks. These background tracks are found in the neutral-current interactions, and they contribute a systematic error for the measurement of the $\nu_{\mu}$ disappearance rate by the MINOS experiment $[58,94]$. The goal of our work is to develop a new $\nu_{\mu}$ charged-current classification algorithm that improves upon the algorithm used for the first MINOS neutrino oscillation analysis [94].

The MINOS experiment records most of its data with the target placed in the low energy beam configuration (see Section 2.2). In this beam configuration, the $\nu_{\mu}$ charged-current interactions produce muons with momentum

peaked around $2 \mathrm{GeV}$ (see Figure 3.4). The muons with $2 \mathrm{GeV}$ momentum are easily distinguished by a long track through the multiple detector planes. An experimental challenge is to identify low momentum muons that form short reconstructed tracks. For example, a typical $1 \mathrm{GeV}$ muon travels through 
approximately 20-30 detector planes.

Many short reconstructed tracks are a part of a hadronic shower with track-like features. These non-muon tracks are produced in neutral-current interactions and, if not correctly identified, may lead to mis-classification of an event as a $\nu_{\mu}$ charged-current interaction. The second most significant source of the non-muon tracks are $\nu_{\mu}$ charged-current events in which a large fraction of the neutrino energy is transfered to a hadronic shower. In these events, the muons are obscured by a hadronic shower causing these events to be essentially indistinguishable from the neutral-current events. As a result, reconstructed tracks in these events do not have the characteristics of a muon track.

In this chapter, a $\nu_{\mu}$ charged-current event classification method based on a new muon identification technique is introduced. This technique uses four track variables that serve as inputs to a multi-variate classification algorithm. First, the reconstructed muon tracks and non-muon tracks in the MC simulation of the near detector were examined. Then, four variables that quantify the differences between muon and background tracks were defined. These four variables were then used to construct a single discriminant variable, which can be interpreted as the probability that a reconstructed track results from a muon track. The discriminant variable was then used to classify events as either $\nu_{\mu}$ charged-current or neutral-current interactions. Finally, the event classification methods for the data and MC events were compared, and the systematic error resulting from the disagreements between the data and MC 
simulation was estimated.

\subsection{Reconstructed muon and non-muon tracks}

This section provides a framework for the subsequent analysis of reconstructed muon and non-muon tracks in the MINOS detectors. First, a muon track term is defined. A number of different particles may deposit energy in a single scintillator strip. The resulting detector hit is a sum of contributions from many particles. In the MC simulation, each energy deposit is tagged with a unique code for the particle associated with that energy deposit. A reconstructed track is considered to be a true muon track if the majority of the hits in this reconstructed track include contributions from the same simulated muon; otherwise, it is considered to be a non-muon (hadronic or background) track. Similarly, a reconstructed event is considered to be a true $\nu_{\mu}$ chargedcurrent event if the majority of the hits in this reconstructed event include contributions from the same simulated $\nu_{\mu}$ charged-current interaction.

Two requirements were implemented as a preselection for the reconstructed tracks to be considered in the analysis. First, only tracks that have at least 5 scintillator plane hits in each detector view were considered. For tracks that failed this requirement, no features were found that would allow

for a distinction between muon and non-muon tracks. The majority of the very short tracks that fail this requirement result from hadronic showers. Approximately $2 \%$ of the reconstructed muon tracks in the MC simulation do not satisfy this requirement. Qualitative observations of the true $\nu_{\mu}$ charged- 
current events that fail this requirement indicate that these muon tracks are obscured by the associated hadronic shower. The second preselection requires that a track vertex (see Section 3.1 for a definition) is contained within the fiducial volume of the detector. The fiducial volume is defined in Section 6.2 for the near detector and in Section 8.2 for the far detector.

In this chapter, the fully calibrated channel response is used. The recorded signal (pulse height) is measured using mip, which a unit that represents the response of the average scintillator strip to a minimum ionizing particle traveling perpendicular to a scintillator plane [70].

\subsection{Improving sensitivity to muon tracks}

The presence of a muon is the signature of $\nu_{\mu}$ charged-current interactions. In these interactions, the muons are produced in association with the hadronic shower. Figure 4.1 shows a $\nu_{\mu}$ charged-current event and neutralcurrent events that contain a reconstructed track. As illustrated in this figure, a vertex of muon tracks is often buried among the detector hits generated by the hadronic shower. Figure 4.2 shows the mean pulse height (signal) of track segments plotted as a function of the track length. These plots illustrate that, in a few planes closest to the track vertex, the muon and non-muon tracks have comparable signal values.

A muon ionization energy loss and the interaction of hadronic showers with a detector are different processes. The main goal of this chapter is to

distinguish these two processes using the pulse height and the topology of 

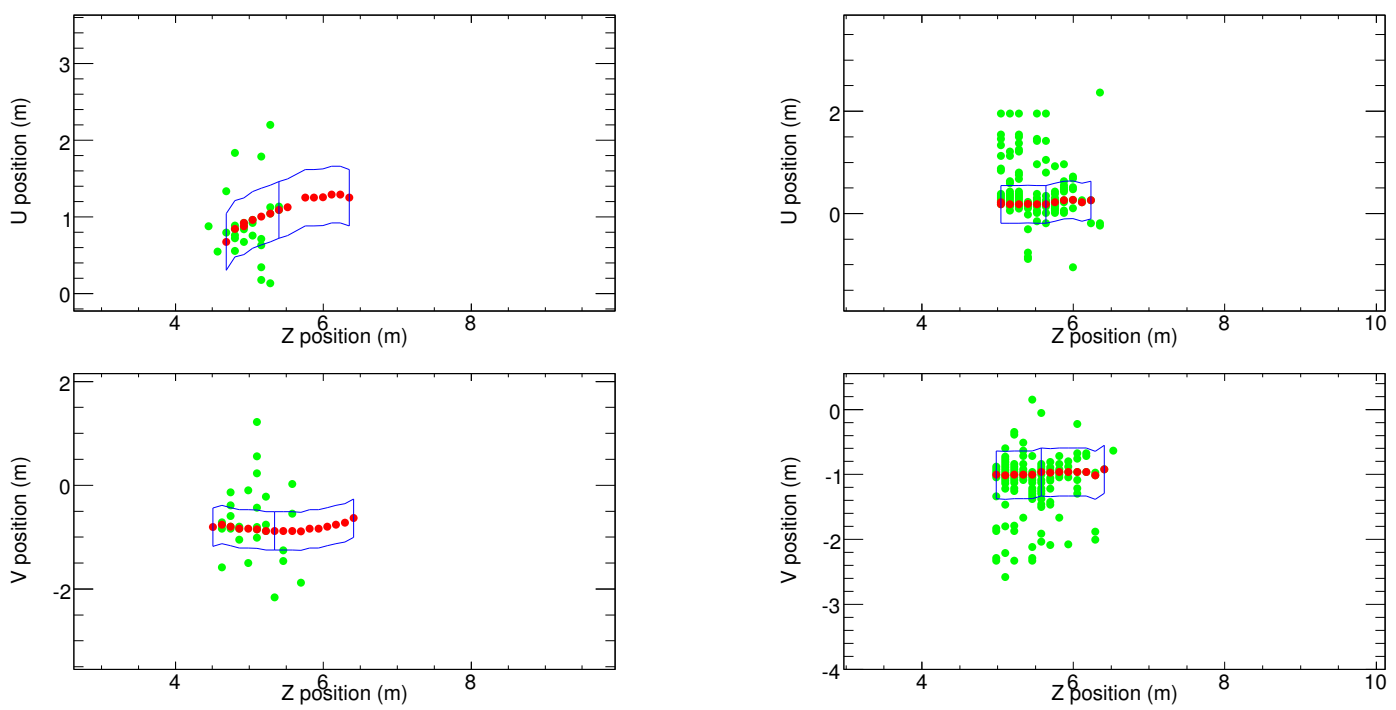

Figure 4.1: Event displays show two reconstructed events from the MC simulation. The left figure shows a true $\nu_{\mu}$ charged-current event, and the right figure shows a true neutral-current event. The reconstructed track hits are shown in red; the reconstructed shower hits are shown in green. For this $\nu_{\mu}$ charged-current event, the (green) shower hits obscure the beginning (vertex) of the track. The track is split into two segments, outlined by the blue boxes, and only the strips from the second (right) segment are used to construct the variables defined in Section 4.3. 


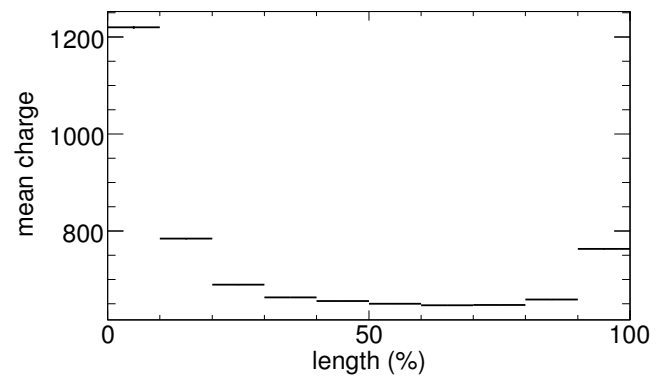

(a)

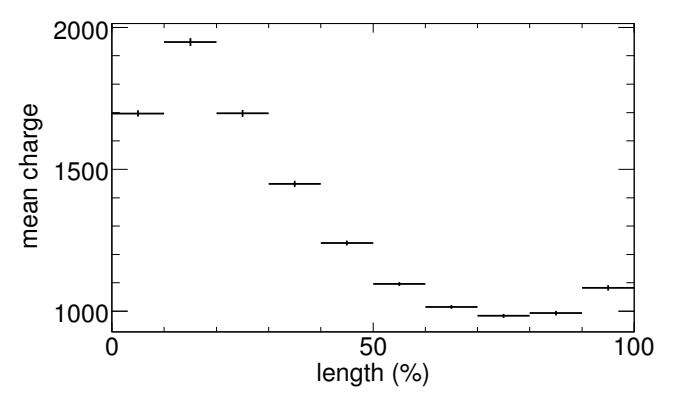

(b)

Figure 4.2: A longitudinal track profile for muon and non-muon reconstructed tracks in the MC simulation. Figure (a) shows muon tracks, and Figure (b) shows non-muon tracks. The tracks are split into ten segments of approximately equal lengths. For each segment, the mean signal of all strips in the segment is computed. The mean signal of the segment is plotted versus the segment position along the track. The units of the $\mathrm{Y}$ axis are ADC counts, where $100 \mathrm{ADC} \approx 1$ photoelectron.

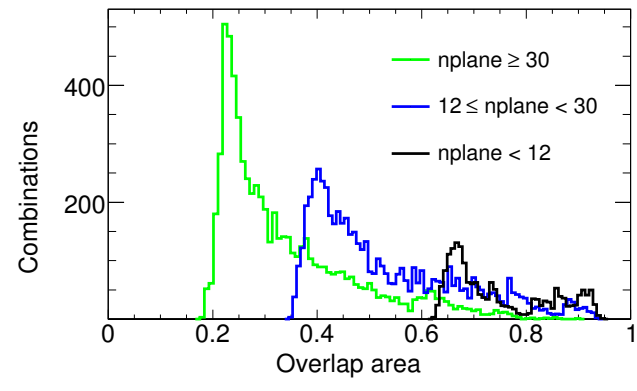

(a)

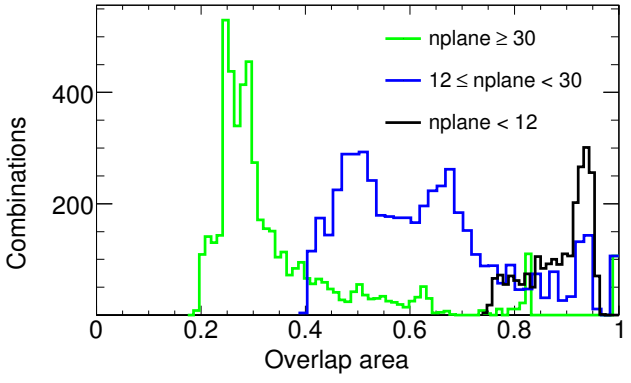

(b)

Figure 4.3: This figure shows an overlap area used to optimize the selection variables, as discussed in the text. Shown are the overlap areas for combinations of parameters; each combination is used to compute the selection variables for the tracks of different lengths. Figure (a) shows the overlap areas for the signal fluctuation variable (defined in Section 4.3.3). Figure (b) shows the overlap areas for the transverse profile variable (defined in Section 4.3.4). A combination of parameters that gives the smallest overlap area for medium length (12-30 planes) tracks was selected for the analysis. 
detector hits. Removing the hadronic shower hits closest to the track vertex might improve a muon identification algorithm. The variables defined in this chapter can be computed for a whole track or only for a part of the track away from the track vertex. A fraction of these excluded track planes is a tunable parameter, which can be varied in order to optimize the muon identification algorithm.

For three of the four variables defined in the next section, an optimization procedure, which maximized the sensitivity to the signature of muon tracks, was employed. For each variable, several parameters were adjusted, such that changing these parameters altered distributions of the considered variable for the muon and non-muon tracks. A figure of merit was defined as a separation between the muon and non-muon distributions measured by an overlap area (an integral of the common area) between these distributions. All available parameters were varied in this fashion to minimize the figure of merit for the three variables described in Section 4.3. Figure 4.3 shows the overlap values for two of the three variables.

\subsection{Track based muon identification variables}

Four variables were constructed to distinguish muon tracks from nonmuon tracks. The first variable is the length of a reconstructed track; this is a good indicator of whether the track is a muon. The pattern of the muon energy loss in the scintillator strips provides another muon track signature. The next two variables use the pattern of the muon energy loss. The fourth variable 
measures an isolation of the muon track from the associated hadronic shower. These four figures are discussed in the following four subsections. Qualitative observations indicate that these variables include most of the information available in the MINOS detectors for muon identification.

Some muons lose all of their energy and stop within the detector, while others exit through the back or sides of the detector. An average muon energy loss does not change significantly for the exiting muons with the momentum in the $1 \mathrm{GeV}$ to $20 \mathrm{GeV}$ range [5]. For the stopping muons, there is a significant increase in the ionization energy loss in the scintillator strips near the end of a track [70]. The left plot in Figure 4.2 shows an increase in the mean pulse height of the last track segment. A variable sensitive to this signature was developed [95]. However, this variable was sensitive to differences between the data and MC simulation and included information largely contained by the other variables. Therefore, adding this variable did not significantly improve the muon identification algorithm.

The distributions of each of the four variables are shown in a set of two figures. One figure shows the distributions for the muon and non-muon tracks from the MC simulation. A second figure shows the distributions for the three categories of MC events: (a) $\nu_{\mu}$ charged-current events with a reconstructed track where the track matches a true muon; (b) $\nu_{\mu}$ charged-current events with a reconstructed track where the track does not match a true muon; (c) neutralcurrent events. ${ }^{1}$ This second figure highlights the earlier argument that the

\footnotetext{
${ }^{1}$ For events with two or more tracks the track with a greatest number of scintillator
} 
neutral-current events and $\nu_{\mu}$ charged-current events without a reconstructed muon track are essentially indistinguishable. In some neutral-current events, a muon is produced in a hadronic shower. Based on the MC simulation, the number of these neutral-current events is negligibly small, and these events are always assigned to the neutral-current events. All histograms were normalized to the unit area.

\subsubsection{Number of track scintillator planes}

The number of track scintillator planes is plotted in Figure 4.4. This variable is proportional to the length of the muon track within the detector. Muons can travel a long distance through matter (compared with hadrons), so long reconstructed tracks are easily identified as muon tracks.

The number of track scintillator planes is a variable that measures the range of the reconstructed track within the detector; the range measures muon momentum if the muon stops within the detector. Because the reconstructed neutrino energy is a function of the reconstructed muon momentum, using this variable has the potential to produce a bias toward selecting neutrinos with energies similar to those in the MC simulation. Two factors significantly reduce this bias. First, a multidimensional likelihood method (described in Section 4.4) is employed for an event classification; this method compares reconstructed tracks of a similar length. Second, a muon carries only a fraction of the total reconstructed neutrino energy, where the fraction is determined

planes is used. 
by the kinematics of the individual neutrino interaction.

\subsubsection{Mean pulse height of track hits}

The mean pulse height of track hits measures the average energy loss in the MINOS scintillator strips. This variable is computed using hits located away from the track vertex. The above-mentioned optimization procedure was used to determine the fraction of the excluded planes. The mean pulse height variable is computed as follows:

- Exclude $30 \%$ of the track scintillator planes closest to the track vertex;

- Compute the mean pulse height of the track hits in the remaining scintillator planes.

The mean pulse height variable is shown in Figure 4.5. Events with large energy depositions by muons are infrequent; thus, this variable has a

narrow distribution for the muon tracks. A non-muon track often belongs to a hadronic shower, and hadronic showers have high mean energy depositions in the scintillator strips, as illustrated in Figure 4.5.

\subsubsection{Signal fluctuation}

The third variable, $R_{f}$, measures fluctuations in the energy deposited in the MINOS scintillator strips. Hadronic showers have larger fluctuations in deposited energy, than muons. This fact is illustrated by the width of the distributions in Figure 4.5. 


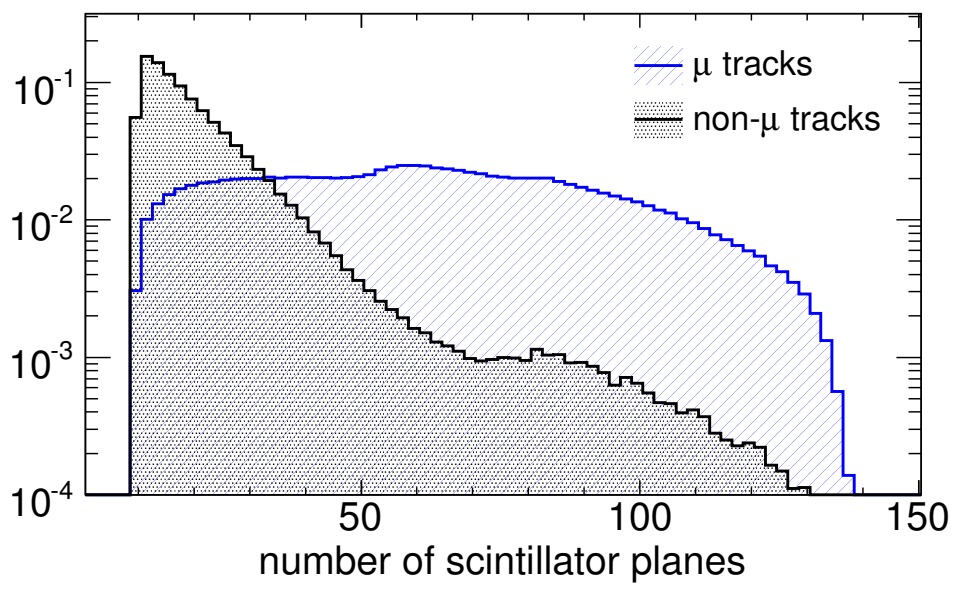

(a)

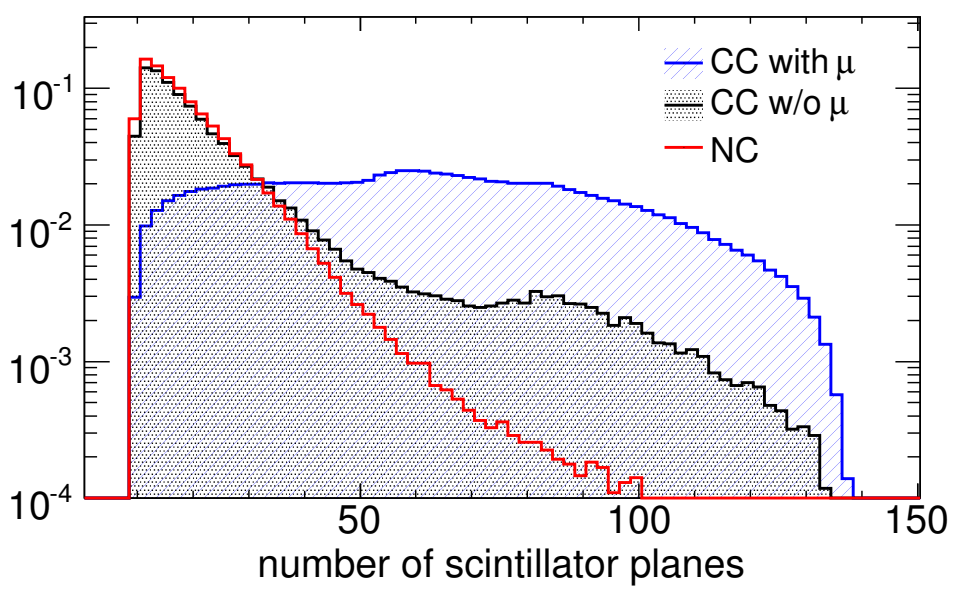

(b)

Figure 4.4: Number of track scintillator planes. Figure (a) shows the reconstructed muon and non-muon tracks. Figure (b) shows the three categories of events, as discussed in the text. 


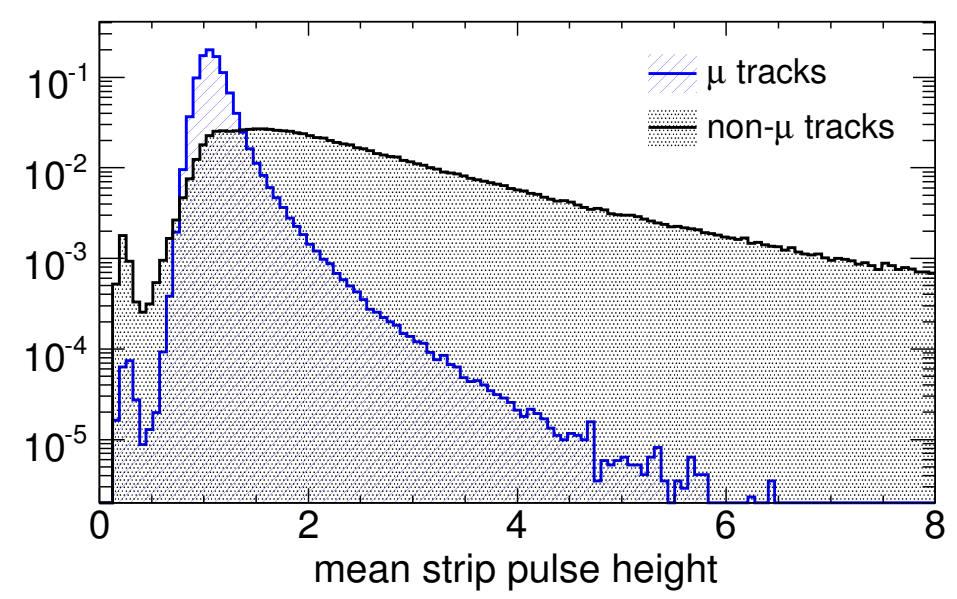

(a)

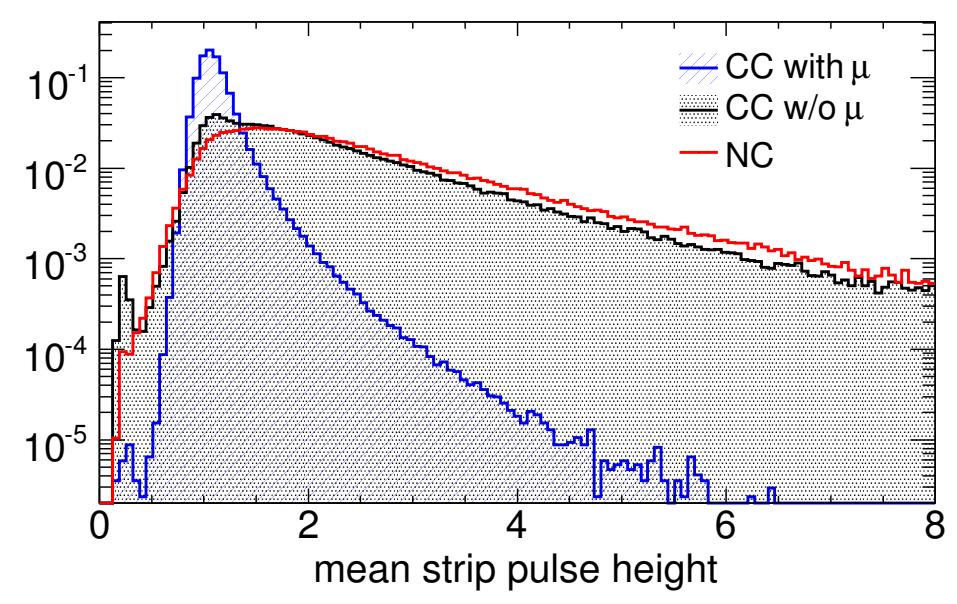

(b)

Figure 4.5: A mean pulse height of the track hits. Figure (a) shows the reconstructed muon and non-muon tracks. Figure (b) shows the three categories of events, as discussed in the text. 
The following four adjustable parameters determine the signal fluctuation variable: (a) the fraction of excluded planes; (b) the number of strips in the window around a track; (c) the time span of the window around a track; (d) the fraction of low pulse height hits to high pulse height hits. The values of these parameters are determined using the optimization procedure described in Section 4.2. The signal fluctuation variable is computed as follows:

- Exclude $30 \%$ of the track scintillator planes closest to the track vertex;

- In the remaining scintillator planes with track hits, select all the detector hits, $S_{i}$, that fall within a 4 strip window and within a 37.36 ns time window around the track hits. Denote $N$ as the number of the selected hits;

- Sort the selected hits in ascending order by pulse height. Divide the sorted hits into two parts: low pulse height hits and high pulse height hits. The fraction where the two parts are divided is a tunable parameter used for the sensitivity optimization procedure;

- Compute the mean of the low pulse height hits and the mean of the high pulse height hits. These two means are shown in Figure 4.6. A signal fluctuation variable is a ratio of the mean of the low pulse height hits over the mean of the high pulse height hits:

$$
R_{f}=\frac{\frac{1}{M} \sum_{i=1}^{M} S_{i}}{\frac{1}{N-M} \sum_{j=M+1}^{N} S_{j}},
$$




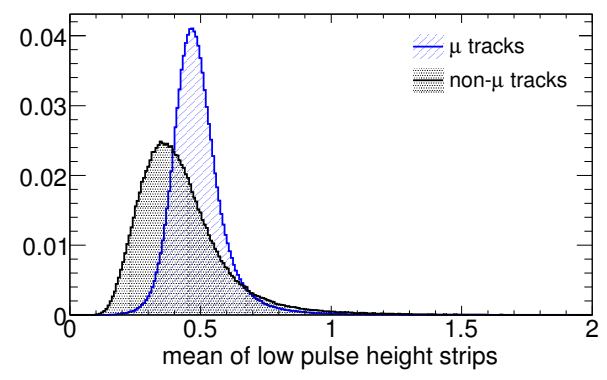

(a)

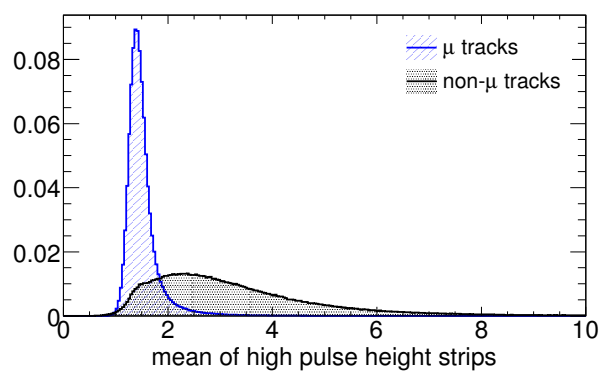

(b)

Figure 4.6: Figure (a) shows a mean of the low pulse height hits. Figure (b) shows a mean of the high pulse height hits.

where $S_{i}$ is the hit pulse height; $S_{i}<S_{j}$, where $1<i \leq M, M<j \leq N$.

$N$ is the number of detector hits, and $M=$ is an integer part of $N / 2$.

The signal fluctuation variable is shown in Figure 4.7. This variable is complimentary to the mean pulse height variable. The peak of the muon distribution is located on the right of the peak of the non-muon distribution. For the muon tracks, the mean of low pulse height hits is approximately $35 \%$ of the mean of high pulse height hits; for the non-muon tracks, this value is approximately $8 \%$.

\subsubsection{Transverse track profile}

The fourth variable is the transverse track profile, $R_{t}$. A scintillator plane is made from closely packed $4.1 \mathrm{~cm}$ wide scintillator strips. A typical muon deposits energy in a single scintillator strip. A typical hadronic shower has a transverse profile a few strips wide, so it deposits energy in multiple 


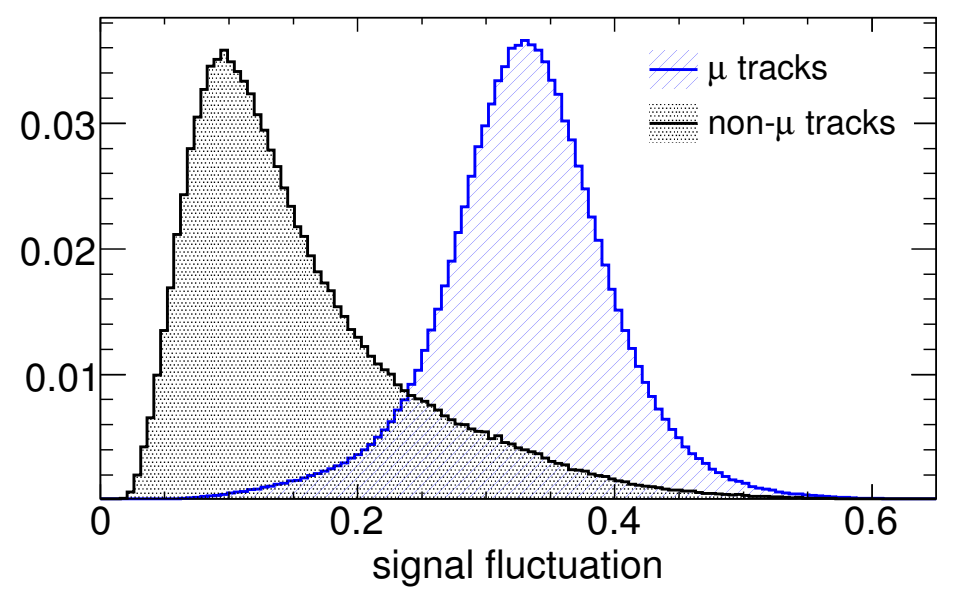

(a)

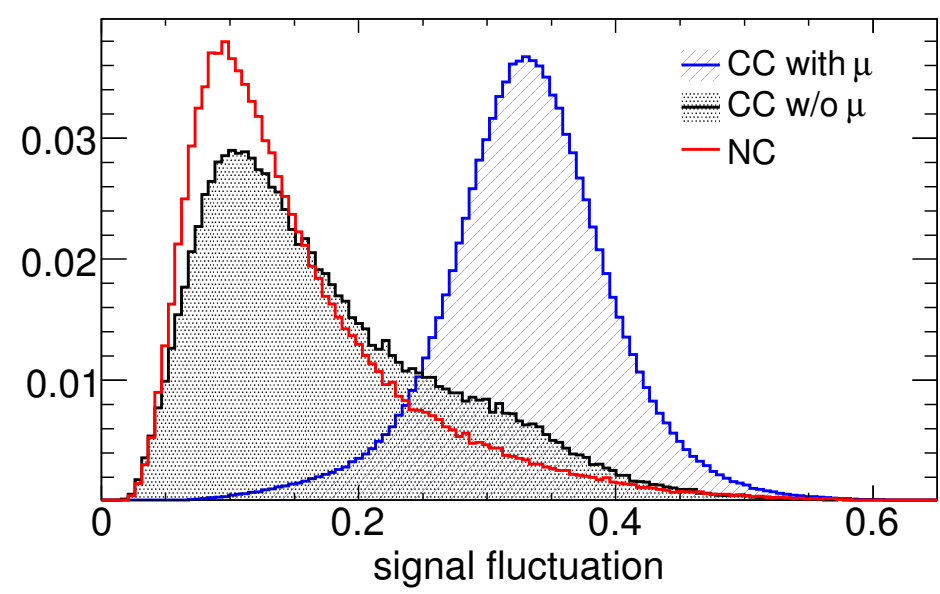

(b)

Figure 4.7: A signal fluctuation variable is the ratio of the mean of low pulse height hits over the mean of high pulse height hits. Figure (a) shows the reconstructed muon and non-muon tracks. Figure (b) shows the three categories of events, as discussed in the text. 
strips within one scintillator plane [72].

The following three adjustable parameters determine this variable: (a) the fraction of the excluded planes; (b) the number of strips in the window around a track; (c) the time span of the window around a track. The values of these parameters were determined using the optimization procedure, described in Section 4.2. The transverse track profile variable is computed as follows:

- Exclude $50 \%$ of the track scintillator planes closest to the track vertex;

- In the remaining scintillator planes with track hits, select all detector hits, $S_{i}^{\text {all }}$, that fall within a 4 strip window and within a $37.36 \mathrm{~ns}$ time window around the track hits, $S_{j}^{\text {track}}$, including hits that belong to the track. Denote $N$ as the number of all the selected hits and $M$ as the number of the track hits;

- The transverse track profile variable is the ratio of the track signal over the signal of all the selected hits:

$$
R_{t}=\frac{\sum_{j=1}^{M} S_{j}^{\text {track }}}{\sum_{i=1}^{N} S_{i}^{a l l}},
$$

where $S_{j}^{\text {track }}$ is the pulse height of the track hits, and $S_{i}^{\text {all }}$ is the pulse height of all the selected hits (including track hits).

The transverse track profile variable is shown in Figure 4.8. This variable compares the pulse height of detector hits in the vicinity of a reconstructed track to the pulse height of the track hits. For muon tracks, this distribution 
has a peak around unity because the muons typically deposit energy in a single scintillator strip.

\subsection{Event classification with the k-nearest neighbor al- gorithm}

An event classification is a decision to assign an event to one of several predetermined classes. A typical classification problem has two classes: signal and background. For example, for an analysis of the $\nu_{\mu}$ oscillations, a physicist assigns reconstructed events to one of the two classes: $\nu_{\mu}$ charged-current interactions or neutral-current interactions. The goal of the classification procedure is to select a maximum number of signal events while keeping a number of background events at a reasonable level, as determined by specifics of a problem.

In the previous section, the four track variables were described. These variables probe the physics of the muon propagation through the MINOS detectors, and they have different distributions for the muon and non-muon tracks. The MC simulation was used to compute probability density functions for these four variables. In general, a set of events with a known class (in this case, a muon or non-muon track) is called a training sample (set). The classification decision is made by comparing a query event ${ }^{2}$ with the events in the training set.

\footnotetext{
${ }^{2}$ The query event is assigned to one of several classes, without knowing its "true" class.
} 


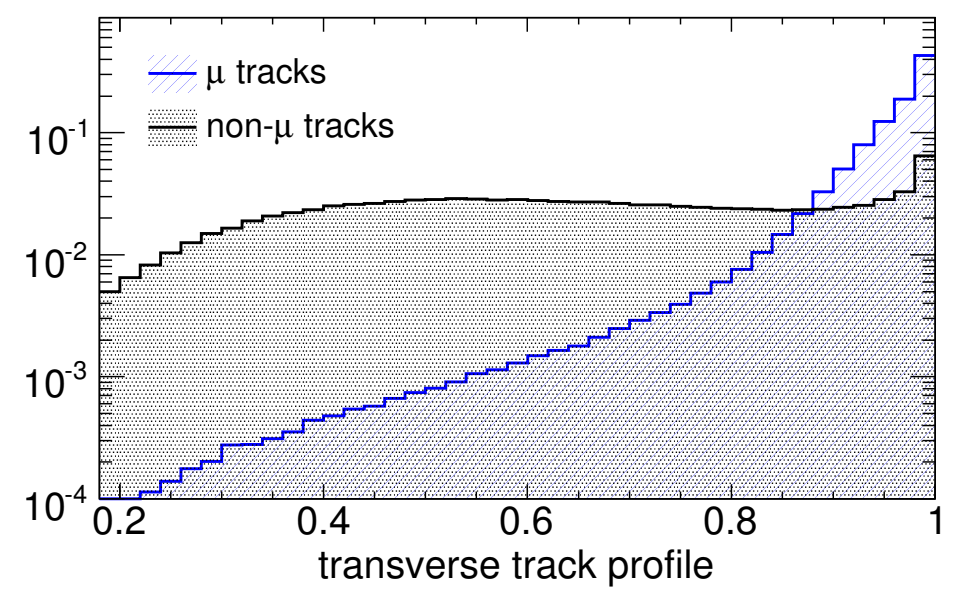

(a)

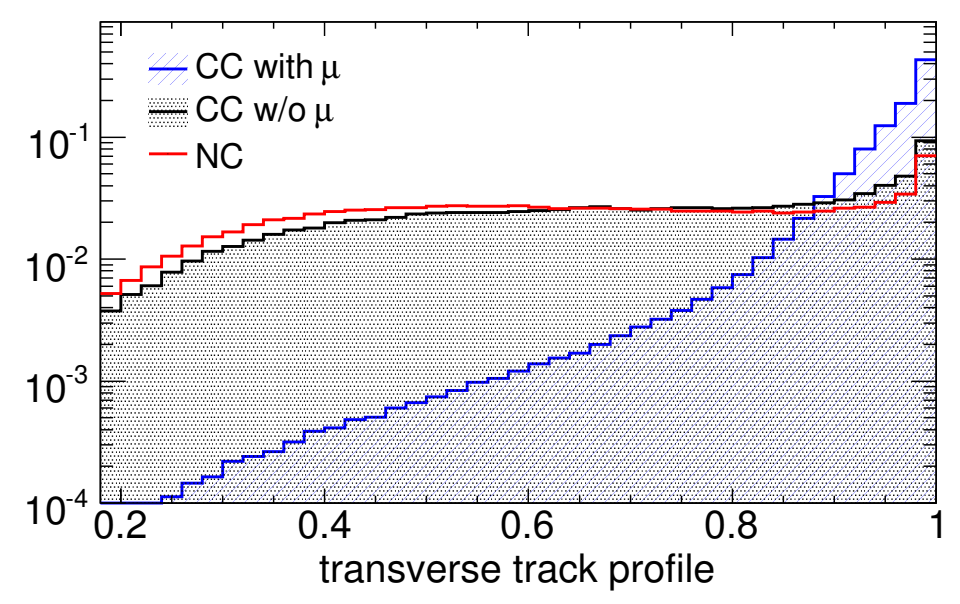

(b)

Figure 4.8: A transverse profile variable. Figure (a) shows the reconstructed muon and non-muon tracks. Figure (b) shows the three categories of events, as discussed in the text. 
The event classification is a statistical problem. In statistics, several methods were developed to compare an event to a training set. The authors of Reference [96] compared a few of these classification algorithms. Based on these comparisons, we decided to use the k-nearest neighbor (knn) algorithm. This algorithm offers easy implementation and high performance [96].

The knn algorithm uses a training set to estimate a density for the signal and background events in a small neighborhood around the query event. The knn algorithm estimates a multidimensional probability density function by counting the number of signal and background events in this small neighborhood. Typically, the signal and background events occupy distinct, but overlapping, regions in the parameter space creating distinguishing features that separate these two classes of events. The knn algorithm finds the $\mathrm{k}$ nearest events from the training set for each query event:

$$
k=k_{S}+k_{B}
$$

where $k_{S}$ is the number of the signal events, and $k_{B}$ is the number of the background events. The probability that the query event is the signal event is approximated by the following expression:

$$
P_{S}=\frac{k_{S}}{k_{S}+k_{B}}=\frac{k_{S}}{k}
$$

This variable is used as a discriminant variable for the event classification.

A vector $X_{i}$ represents a point in a multidimensional parameter space; this vector is constructed using the classification variables for the event $i$. 
Events are compared using a distance function, $D$. This distance function between two events is the Euclidean distance function:

$$
D=\left(\sum_{i=1}^{d}\left|X_{i}^{T}-X_{i}^{Q}\right|^{2}\right)^{\frac{1}{2}}
$$

where $d$ is the number of variables, $X_{i}^{T}$ are the variables of the event from a training set, and $X_{i}^{Q}$ are the variables of the query event. The k events with the smallest values of $D$ are the k-nearest neighbors. A value of $\mathrm{k}$ determines the average size of the neighborhood over which probability density functions are evaluated. Large values of $\mathrm{k}$ do not capture the local behavior of the probability density functions, while small values of $\mathrm{k}$ cause large fluctuations in a classification outcome. An optimal value of $\mathrm{k}$ for this analysis is briefly discussed in the next section.

Figures 4.9 illustrates event classification with the k-nearest neighbor algorithm. The number of events in the training set and the number of variables were reduced to illustrate the principles behind the knn algorithm.

The classification variables are normalized to compensate for different units among the variables. A normalization scale factor for each variable is determined as a width, $w_{i}$, of the combined distribution of the $X_{i}^{T}$ variable for the signal and background events. The width $w_{i}$ is an interval that contains $80 \%$ of the $X_{i}^{T}$ values, where the lower $10 \%$ and upper $10 \%$ of the values are excluded. The $10 \%$ fraction was chosen ad hoc based on qualitative observations about the input distributions. The events in "tails" are thus excluded, because 
outlying events can significantly change the width of the entire distribution. For the normalization, the variable $i$ is scaled by the $1 / w_{i}$ factor.

A total number of signal and background events in the training set depend on a MC physics model. This dependence on the MC simulation is reduced if an equal number of the signal and background events is included into the training set. In the MINOS MC simulation, the number of muon tracks (signal) exceeds the number of non-muon tracks (background) by a factor of 3 . For our analysis, the number of the muon tracks included within the training set was reduced to match the number of non-muon tracks for the following two reasons. First, the number of muon and non-muon tracks is determined by the $\nu_{\mu}$ charged-current and neutral-current cross-sections; including an equal number of the muon and non-muon tracks removes prior knowledge about these cross-sections for the analysis of data events. Second, an equal number of signal and background events creates an algorithm that evenly samples the multi-dimensional parameter space populated by the events from the training set.

A software implementation of the k-nearest neighbor algorithm ${ }^{3}$ stores $N$ training events in a computer memory and searches through these events for the k-nearest neighbors. The following two search functions were developed: a simple search and a search based on a kd-tree structure. For the simple search, a query event is compared to all training events. This search algorithm requires

\footnotetext{
${ }^{3}$ The code developed for the knn algorithm was contributed to the TMVA project [97].
} 

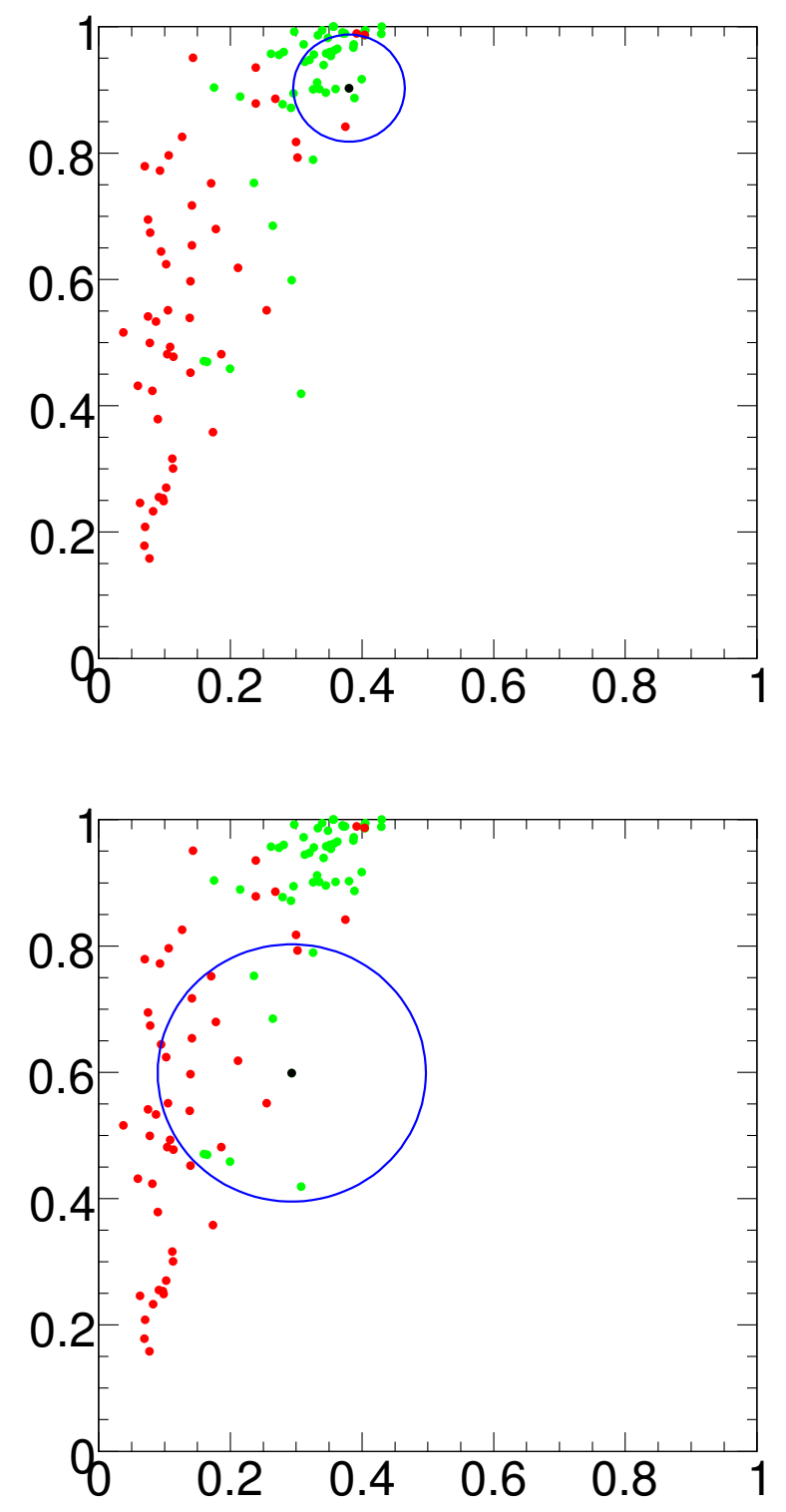

Figure 4.9: Examples of knn classification for two variables. Signal events are shown in green; background events are shown in red; and a query event is shown in black. A neighborhood enclosing the 20 nearest neighbors is shown in blue. Top: the neighborhood contains 19 green points and 1 red point - the query event is classified as a signal event. Bottom: the neighborhood contains 7 green points and 13 red points - the query event is classified as a background event. 
$N$ computations of the distance function, and for a large number of events it becomes impractical. A significant improvement in the performance of the knn algorithm was attained by using the kd-tree data structure [98,99]. This search algorithm requires approximately $\log N$ computations of the distance function. The two search algorithms were tested using the same events, and both algorithms produced identical results, as expected.

\subsection{Identification of muon neutrino charged-current events}

Reconstructed events in the MINOS detectors are classified using information from a reconstructed track. The reconstructed events without a reconstructed track are dominated by the neutral-current interactions. For our analysis, these events are always classified as neutral-current interactions. The reconstructed events that contain more than one track are classified using the longest track. ${ }^{4}$ Approximately $3 \%$ of events have two reconstructed tracks; a number of events with three tracks is less than 1\%; and, there are no events with more than three tracks. A reconstructed event is classified as a $\nu_{\mu}$ charged-current event if the reconstructed track is a muon track; otherwise, it is classified as a neutral-current event. In this section, both $\nu_{\mu}$ charged-current and $\bar{\nu}_{\mu}$ charged-current interactions are included into one category since the track classification variables do not distinguish between $\mu^{+}$and $\mu^{-}$tracks.

The reconstructed tracks are classified using a discriminant variable

\footnotetext{
${ }^{4}$ The longest track has a greatest number of the scintillator plane hits.
} 
computed with the knn algorithm. This variable approximates the probability that a reconstructed track is a muon track, as discussed in Section 4.4. A training set was created using a current version of the MINOS MC simulation. For the near detector, the training set contains 397,516 muon tracks and an equal number of non-muon tracks. For the far detector, the training set contains 163,838 muon tracks and an equal number of non-muon tracks. A classification result has no observable dependency on the size of the training set for sets with that many tracks. For each track in the training set, four classification variables and a true MC type (muon or non-muon) are recorded.

The discriminant variable, $P_{S}$, is a fraction of the muon tracks among the $\mathrm{k}$ nearest neighbors around a query track:

$$
P_{S}=\frac{k_{\mu}}{k_{\mu}+k_{n o n-\mu}}=\frac{k_{\mu}}{k},
$$

where $k_{\mu}\left(k_{n o n-\mu}\right)$ is the number of muon (non-muon) tracks among the $\mathrm{k}$ neighbors. The discriminant variable is shown in Figure 4.10 for the near detector and in Figure 8.6 for the far detector. The events with $P_{S}>0.3$ are identified as the $\nu_{\mu}$ charged-current and $\bar{\nu}_{\mu}$ charged-current candidate events. The same 0.3 value is used for the near and far detectors. This 0.3 value was obtained in a sensitivity study for an analysis of the $\nu_{\mu}$ disappearance [100]. In this MC study, the selection threshold was changed in small steps. For each step, the selected events were analyzed for the $\nu_{\mu}$ charged-current disappearance; selecting events with $P_{S}>0.3$ produced the smallest errors for the oscillation parameters. 


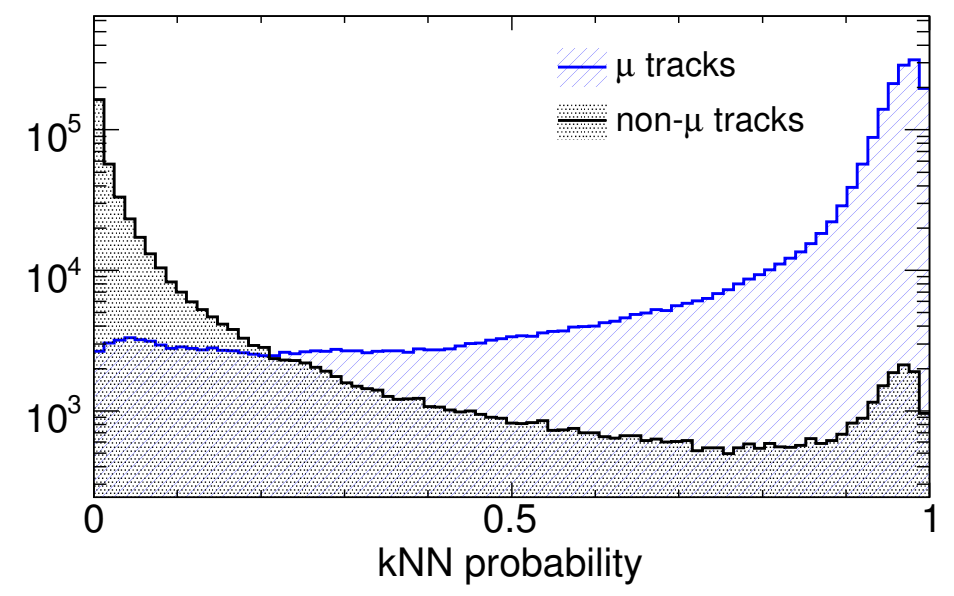

(a)

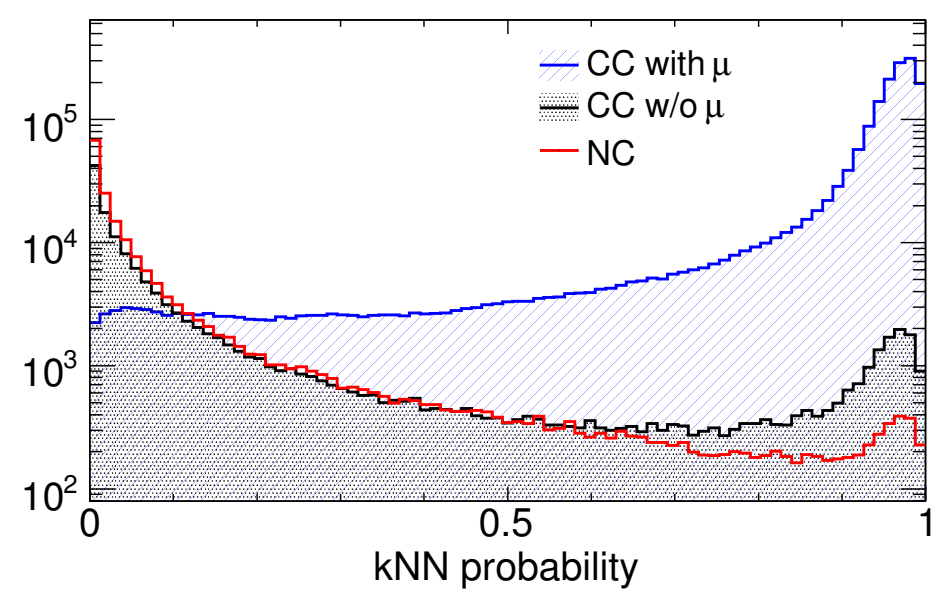

(b)

Figure 4.10: k-nearest neighbor variable for tracks and events. The variables are computed using the 80 nearest neighbors. Figure (a) shows the reconstructed muon and non-muon tracks. Figure (b) shows the three categories of events, as discussed in Section 4.3. 
A k-nearest neighbor algorithm does not specify an optimal value of $\mathrm{k}$ for a given classification problem. The performance of the knn algorithm is evaluated for several values of $\mathrm{k}$ using the following two quantities:

Signal acceptance: the number of selected signal events divided by the total number of signal events before the selection.

Background rejection: the number of rejected background events divided by the total number of background events before the selection.

Figure 4.11 shows the selection efficiency and the background rejection; these two quantities do not depend on $\mathrm{k}$, for $\mathrm{k}=20,60,100$. The analysis uses $k=80$; this value puts a reasonable separation (in a number of histogram bins) between the muon and non-muon peaks in the distributions shown in Figure 4.10.

\subsection{A study of event selection for several beam config- urations}

In this section, the energy dependence of the muon selection efficiency is examined. The $\mathrm{MC}$ events used to create a training set are generated using the MC simulation of the low energy beam configuration. The classification algorithm includes the number of planes variable, which depends on muon momentum. As a result, the classification algorithm contains some information about the MC neutrino energy spectrum. Figure 4.12 shows the efficiency and purity (defined in Section 3.3) for the three beam configurations. The efficiency 


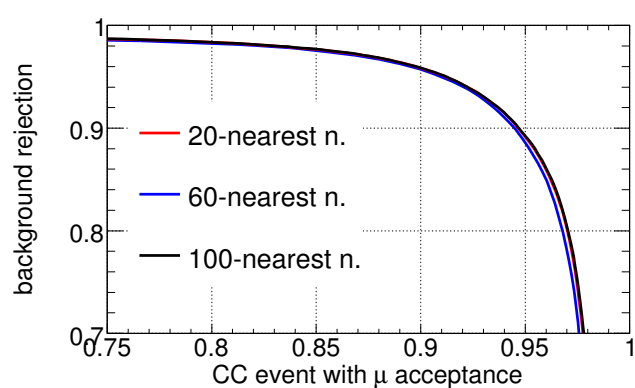

(a)

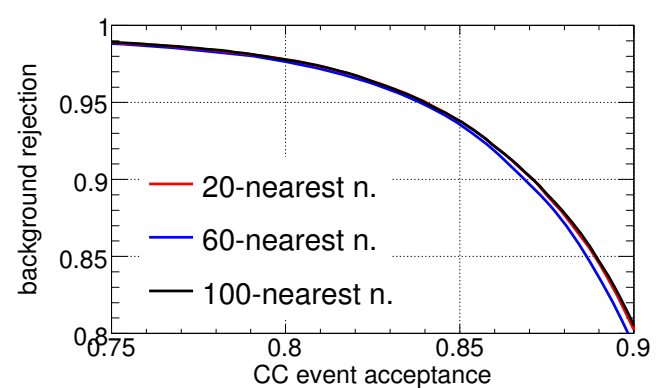

(b)

Figure 4.11: A background rejection is plotted versus a signal acceptance for $\mathrm{k}$ $=20,60,100$. Figure (a) shows the signal acceptance of the $\nu_{\mu}$ charged-current events with a reconstructed track that matches a true muon. Figure (b) shows the signal acceptance of all the $\nu_{\mu}$ charged-current events. The $\nu_{\mu}$ chargedcurrent events are selected using a charge-sign variable, defined in Chapter 5.

is the same for the three configuration, so it does not depend on the MC energy spectrum. The purity for the high energy beam is lower, because the number of neutral-current background events from the high energy tail is increased.

\subsection{Comparison of muon tracks for data and MC}

The excess of low pulse height hits in the near detector data (see Section 3.6) implies differences between the data and MC simulation in topology and pulse height of detector hits. The muon identification method compares a query track to a training set of MC tracks. These differences between the data and the MC simulation can potentially produce different selection results for data and $\mathrm{MC}$ events. This section evaluates a systematic error resulting from the excess of low pulse height hits.

Two selection methods for the near detector tracks are compared: (a) 


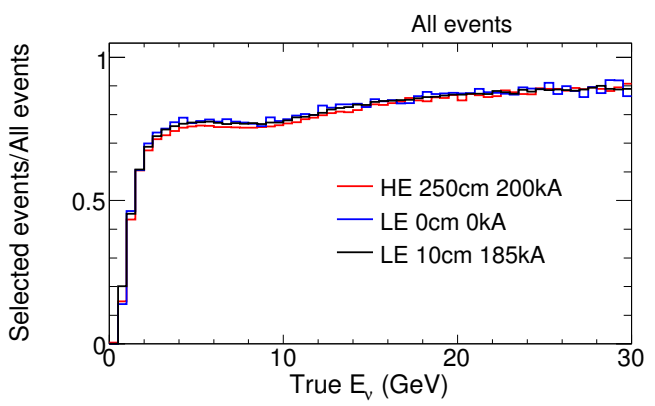

(a)

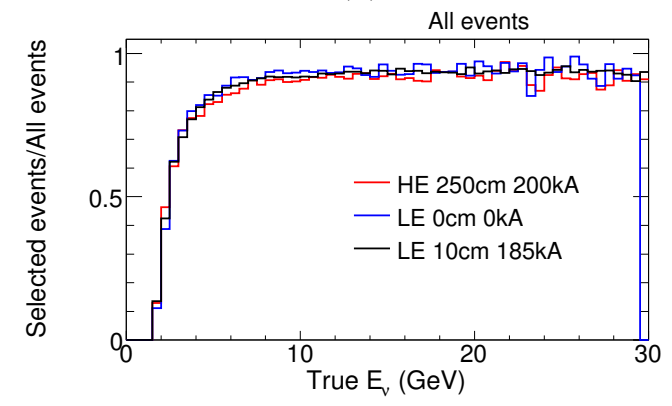

(c)

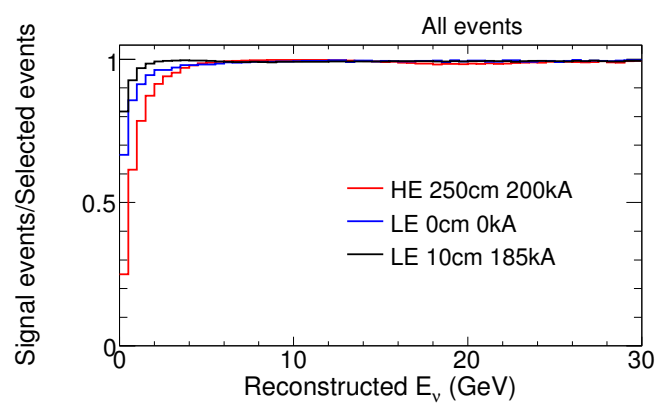

(b)

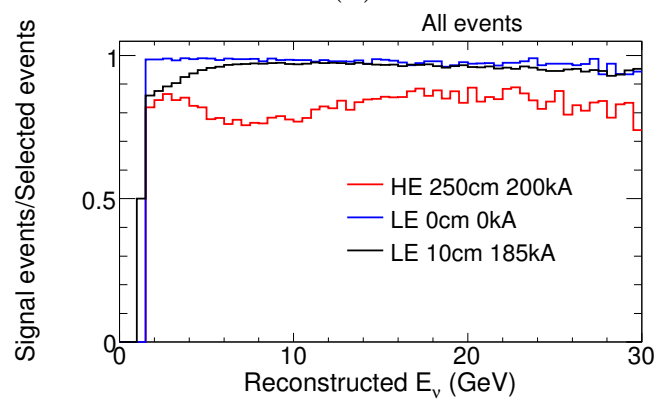

(d)

Figure 4.12: Figure (a) shows the efficiency, and Figure (b) shows the purity for the $\nu_{\mu}$ charged-current selection. Figure (c) shows efficiency, and Figure (d) shows purity for the $\bar{\nu}_{\mu}$ charged-current events. Each figure includes the near detector $\mathrm{MC}$ events from three beam configurations. 
an original method which uses all detector hits; (b) a modified method which uses hits from the calorimeter section with a pulse height greater than $0.3 \mathrm{mip}$ (approximately 1.8 photoelectrons). The original method is sensitive to hits of any pulse height. The modified method uses hits with high pulse height, which are accurately modeled by the MC simulation (see Section 3.6). The spectrometer section of the near detector is excluded because this section is not fully calibrated, due to sparse scintillator coverage. The same modifications are applied in the data and MC simulation. To summarize, the modified method uses only calorimeter hits with a well calibrated, high pulse height signal.

The track finding algorithm is described in Section 3.1. This algorithm uses hits with pulse heights greater than 2 photoelectrons to create track clusters; this requirement significantly reduces the dependence of the track finding algorithm on low pulse height hits. As a result, the excess of low pulse height hits in the near detector data does not affect the results of the algorithm for finding real muon tracks.

Figure 4.13 shows the four classification variables for the original and modified methods. For the original method, the signal fluctuation variable shows significant discrepancy in the right peak (populated by the true muon tracks). The agreement between the data and the MC simulation for the signal fluctuation variable significantly improve with the modified method. The signal fluctuation variable is a ratio of low pulse hits to high pulse hits, so removing additional, not modeled low pulse hits improves the agreement between the data and MC simulation, as expected. 

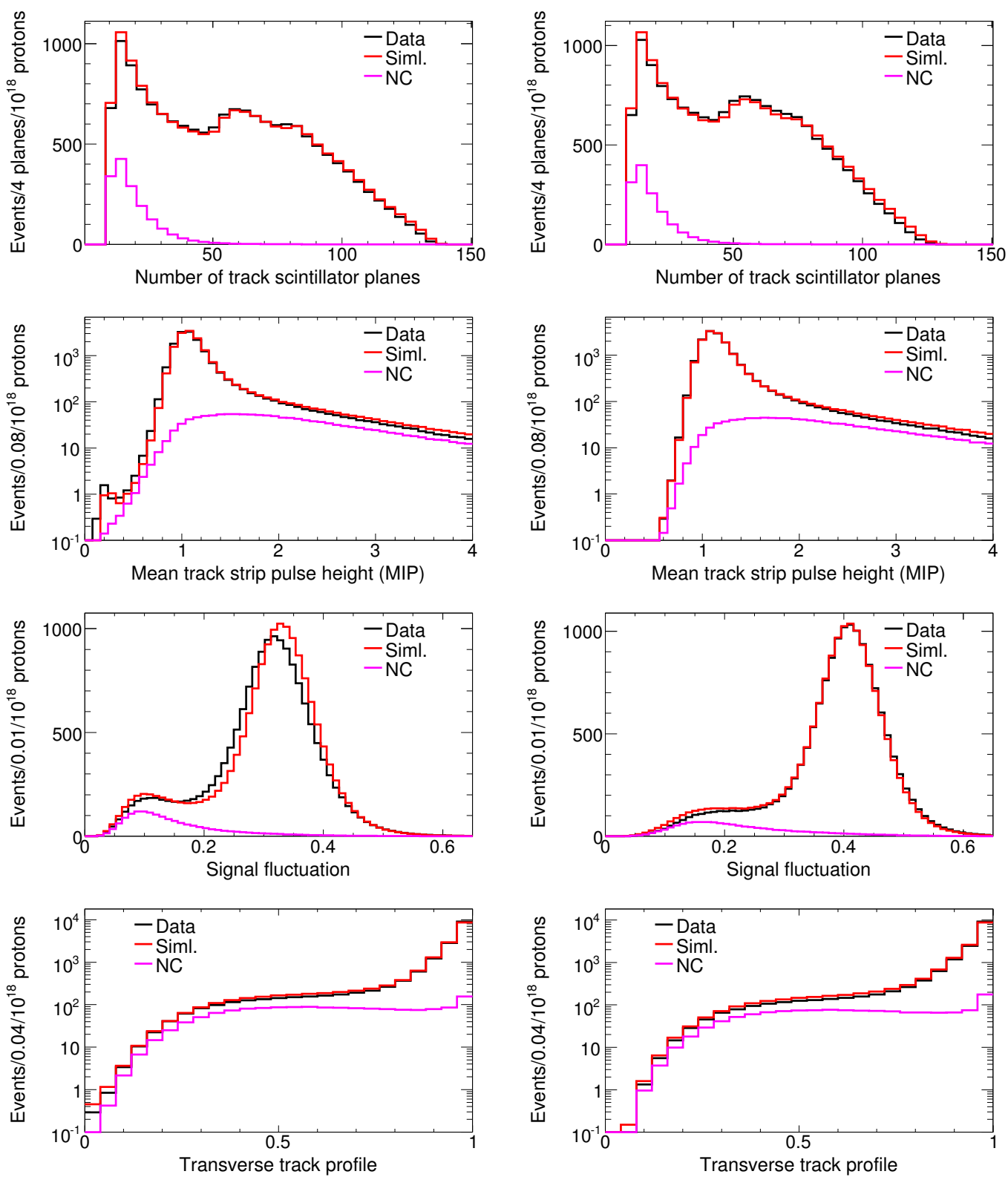

Figure 4.13: Four muon identification variables shown for the near detector data and MC tracks with the original method (left) and the modified method (right), as discussed in the text. The left plots use all near detector strips. The right plots use the calorimeter strips with a pulse height greater than $0.3 \mathrm{mip}$ (approximately 1.8 photoelectrons). The MC histograms (labeled as "Siml.") are normalized to the number of data events. The true MC neutral-current events are shown in light purple. 


\begin{tabular}{l|r|r} 
Data & Modified method: pass & Modified method: fail \\
\hline Original method: pass & $2204565(63.49 \%)$ & $35464(1.02 \%)$ \\
\hline Original method: fail & $22993(0.66 \%)$ & $1209446(34.83 \%)$
\end{tabular}

\begin{tabular}{l|r|r} 
MC & Modified method: pass & Modified method: fail \\
\hline Original method: pass & $3236804(61.28 \%)$ & $50590(0.96 \%)$ \\
\hline Original method: fail & $35662(0.68 \%)$ & $1958766(37.09 \%)$
\end{tabular}

Table 4.1: Number of the near detector data and MC events selected and rejected by the original and modified methods, as discussed in the text.

Figure 4.13 shows the discriminant variable, $P_{S}$, for the original and modified methods. For both methods, the data and MC distributions agree with each other. The $\nu_{\mu}$ charged-current events are selected with $P_{S}>0.3$. There are a few events per bin in this region, which minimizes a possible migration of events across the 0.3 boundary due to MC errors. Figure 4.15 shows the radius of the knn neighborhood with the 80 neighbors. The remaining differences between the data and MC events are consistent with an overall understanding of the muon neutrino flux and cross-sections (see Chapter 7).

A systematic error is computed by comparing the two selection methods. Table 4.1 shows the number of near detector data and MC events with $P_{S}>0.3$ (pass) and $P_{S}<0.3$ (fail). The number of the selected $\nu_{\mu}$ chargedcurrent data events is greater than the number of MC events, consistent with the results of tuning muon neutrino flux and cross-sections models in Chapter 7. A fraction of events that pass the original method and fail the modified method, and vice versa, is consistent with both the data and the MC simu- 


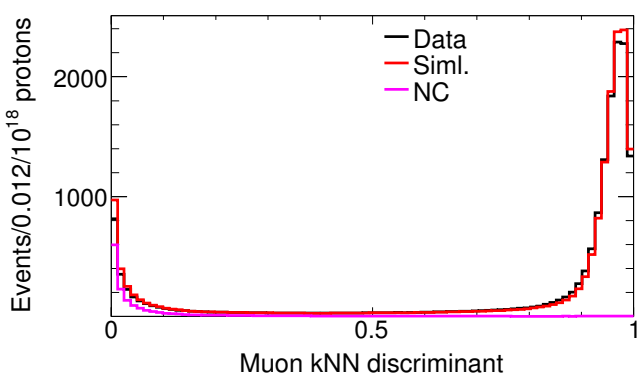

(a)

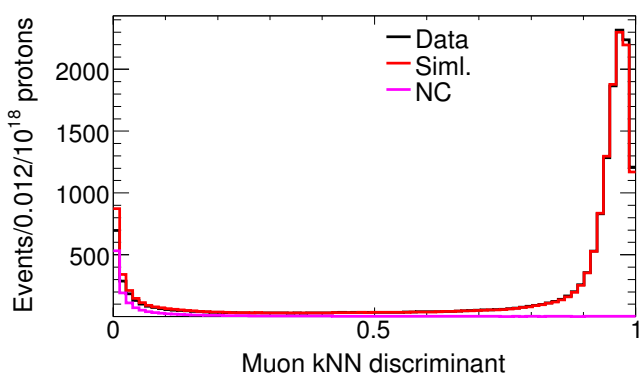

(c)

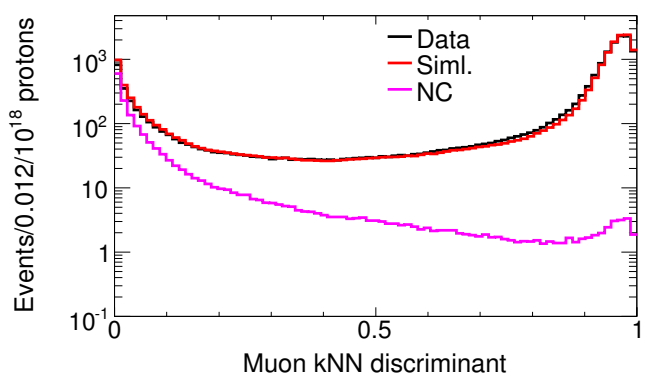

(b)

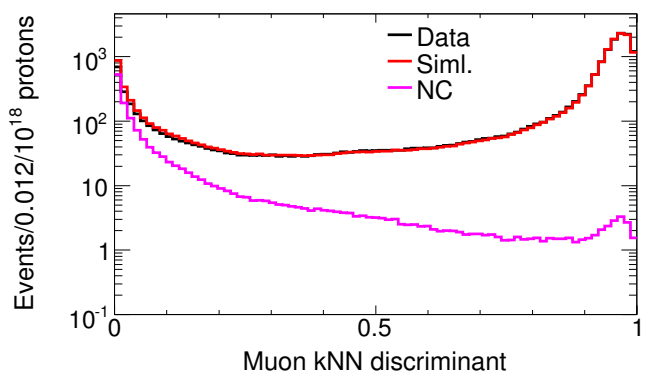

(d)

Figure 4.14: A discriminant variable shown for the near detector data and MC simulation. Figures (a) and (b) use all detector strips (original method). Figures (c) and (d) use strips from the calorimeter section with a pulse height greater than 0.3 mip (modified method). The MC histograms are normalized to the number of data events. The true MC neutral-current events are shown in light purple. 

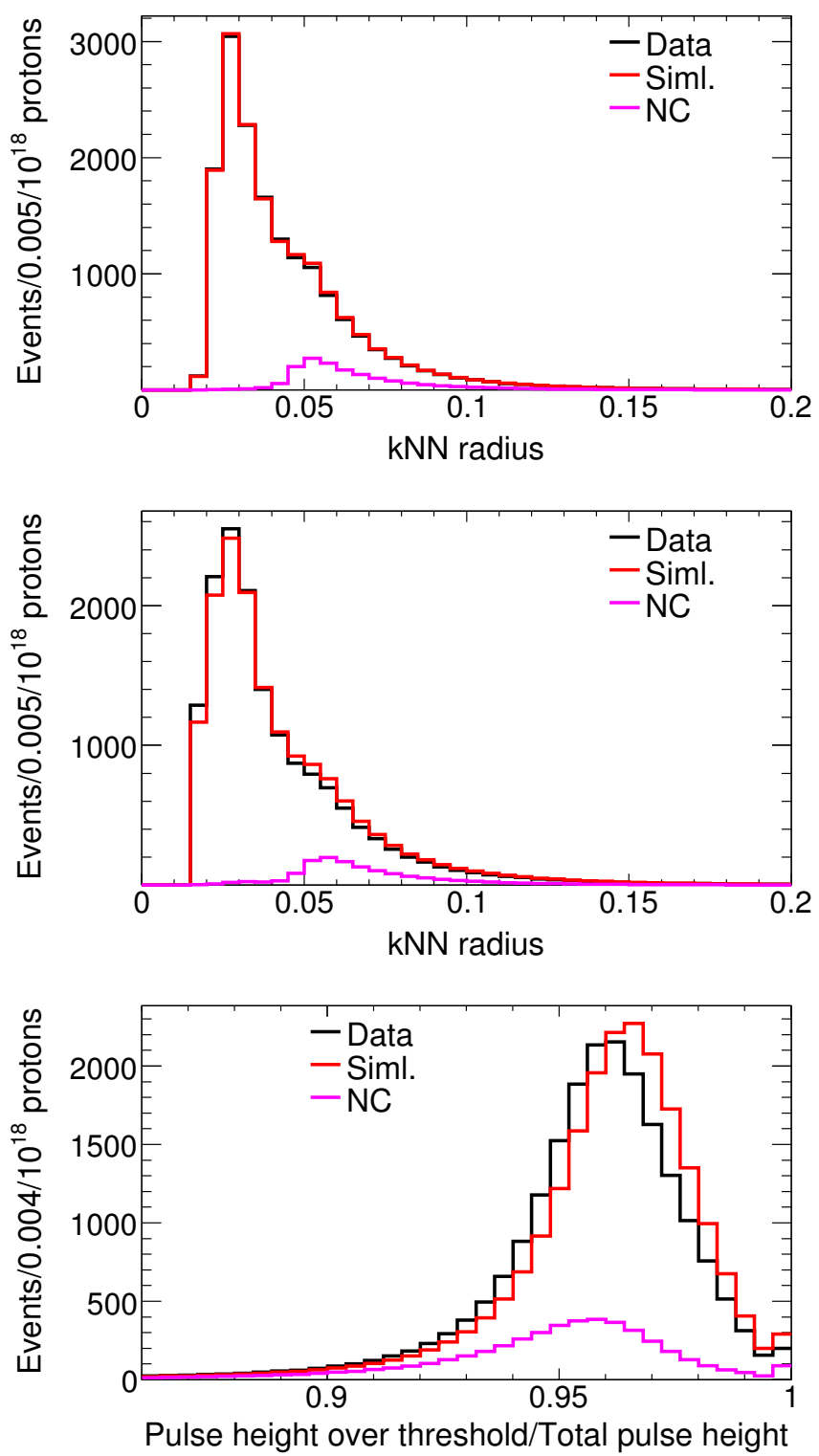

Figure 4.15: The top two figures show the radius of the knn neighborhood for the near detector data and the MC simulation for the original method (top) and for the modified method (middle), as discussed in the text. The bottom figure shows the fraction of the event pulse height contained within the high pulse height hits (with a pulse height greater than 2 photoelectrons). This figure shows all reconstructed events contained within the near detector fiducial volume. The MC histograms are normalized to the number of data events. The true MC neutral-current events are shown in light purple. 
lation. Based on the numbers in Table 4.1, the systematic error on the total number of selected $\nu_{\mu}$ charged-current data events is at most $0.5 \%$. This error is estimated as the difference between the number of data events that pass one method but fail another (difference between the off-diagonal elements for the data events in Table 4.1).

There is an additional systematic error associated with an excess of low pulse height hits in the data. A reconstructed shower energy is tuned using the $\mathrm{MC}$ simulation. On average, the reconstructed shower energy matches the true hadronic energy, as shown in Section 3.4. The excess of low pulse height hits in the data can generate a systematic shift for a measurement of the shower energy for data events. Figure 4.15 shows a fraction of the event pulse height contained in hits with a pulse height greater than 2 photoelectrons (high pulse height hits). These high pulse height hits are reasonably well reproduced by the MC simulation (see Section 3.6). Approximately $96.5 \%$ of the event pulse height is contained in the high pulse height hits. The difference between the data and the MC simulation is less than $1 \%$. This systematic error is sigficantly smaller than the $10 \%$ systematic error on the absolute shower energy measurement in the MINOS detectors [58].

\subsection{Summary}

In this chapter, a new method to the select $\nu_{\mu}$ charged-current and $\bar{\nu}_{\mu}$ charged-current events in the MINOS detectors was introduced. This method uses the k-nearest neighbor algorithm and the four track based variables. The 
selected $\nu_{\mu}$ charged-current events at the far detector include the estimated $0.6 \%$ background contamination from the neutral-current interactions [46]. This is compared to the $1.8 \%$ contamination for the previous analysis [58]. A smaller background contamination reduces systematic errors for the measurement of the muon neutrino oscillation parameters [46].

The near detector records the excess of low pulse height hits, compared to the MC simulation. This excess can potentially introduce a systematic error for selecting $\nu_{\mu}$ charged-current events. This systematic error was evaluated in this chapter; it is less than $0.5 \%$. The near and far detectors have a similar response to physical detector hits, so these results are appliacable to far detector analysis.

The MINOS detectors are magnetized, and thus the muon charge sign is determined by measuring the curvature of a reconstructed track, as discussed in Section 3.1. However, the four classification variables do not depend on the track curvature. As a result, the muon identification algorithm also does not depend on the track curvature. The algorithm developed in this chapter can be used to select both $\nu_{\mu}$ charged-current and $\bar{\nu}_{\mu}$ charged-current events. A measurement of the muon charge sign is the subject of Chapter 5 . 


\section{Chapter 5}

\section{Measurement of muon charge sign}

The MINOS detectors are made of magnetized steel so that charged particles traveling through the detector bend in the magnetic field. The muon charge sign is determined using a curvature of the muon track in the magnetic field. A track fitting algorithm measures the muon momentum and muon charge sign, by using a pattern of track hits and a detailed map of the detector magnetic field $[52,74]$.

Muon neutrino charged-current interactions are identified by an observation of a $\mu^{-}$track. Muon anti-neutrino charged-current interactions are identified by an observation of a $\mu^{+}$track. The $\mu^{-}$and $\mu^{+}$tracks are identified by a sign of the track curvature in the magnetic field. In addition to the magnetic field effects, muons also experience multiple Coulomb scattering, which produces small random changes in the muon's trajectory. These changes can generate errors when measuring the muon charge sign. The error of assigning a positive charge sign to a true $\mu^{-}$track and the error of assigning a negative charge sign to a true $\mu^{+}$track are similar in magnitude, as illustrated in Figure 5.1.

For the low energy beam, the predicted neutrino spectrum at the near 
detector is composed of $92.9 \% \nu_{\mu}, 5.8 \% \bar{\nu}_{\mu}$, and $1.3 \% \nu_{e}+\bar{\nu}_{e}[58]$. Because there are many more $\nu_{\mu}$ events, compared to $\bar{\nu}_{\mu}$ events, the standard algorithm is augmented to improve the separation of the $\bar{\nu}_{\mu}$ charged-current and $\nu_{\mu}$ chargedcurrent events.

In this chapter a new variable is described, which is used to improve the measurement of the muon charge sign. First, the performance of the track fitting algorithm is briefly discussed. Then, the new charge-sign variable is constructed. It is shown that this variable is sensitive to the muon charge sign and that it reduces a number of the background $\nu_{\mu}$ charged-current events, among the selected $\bar{\nu}_{\mu}$ charged-current events.

\subsection{Charge-sign measurement using fitting algorithm}

The MINOS detectors are muon tracking detectors; muons leave visible tracks in the detectors, as illustrated in Figure 4.1. The track finding and track fitting algorithms are described in Section 3.1. The track fitting algorithm computes the following three variables: (a) the muon momentum $p$ at a neutrino interaction vertex; (b) the muon charge sign $q$; (c) the $\sigma_{q / p}$ error (this is the error on the $q / p$ ratio). The first two variables are computed together as the $q / p$ ratio.

The fitting algorithm assumes that the reconstructed track is due to a muon. Non-muon tracks are identified and rejected using the muon identification algorithm described in Chapter 4. For some tracks, the $q / p$ ratio cannot be unambiguously computed; such tracks are assigned a "failed fit" 

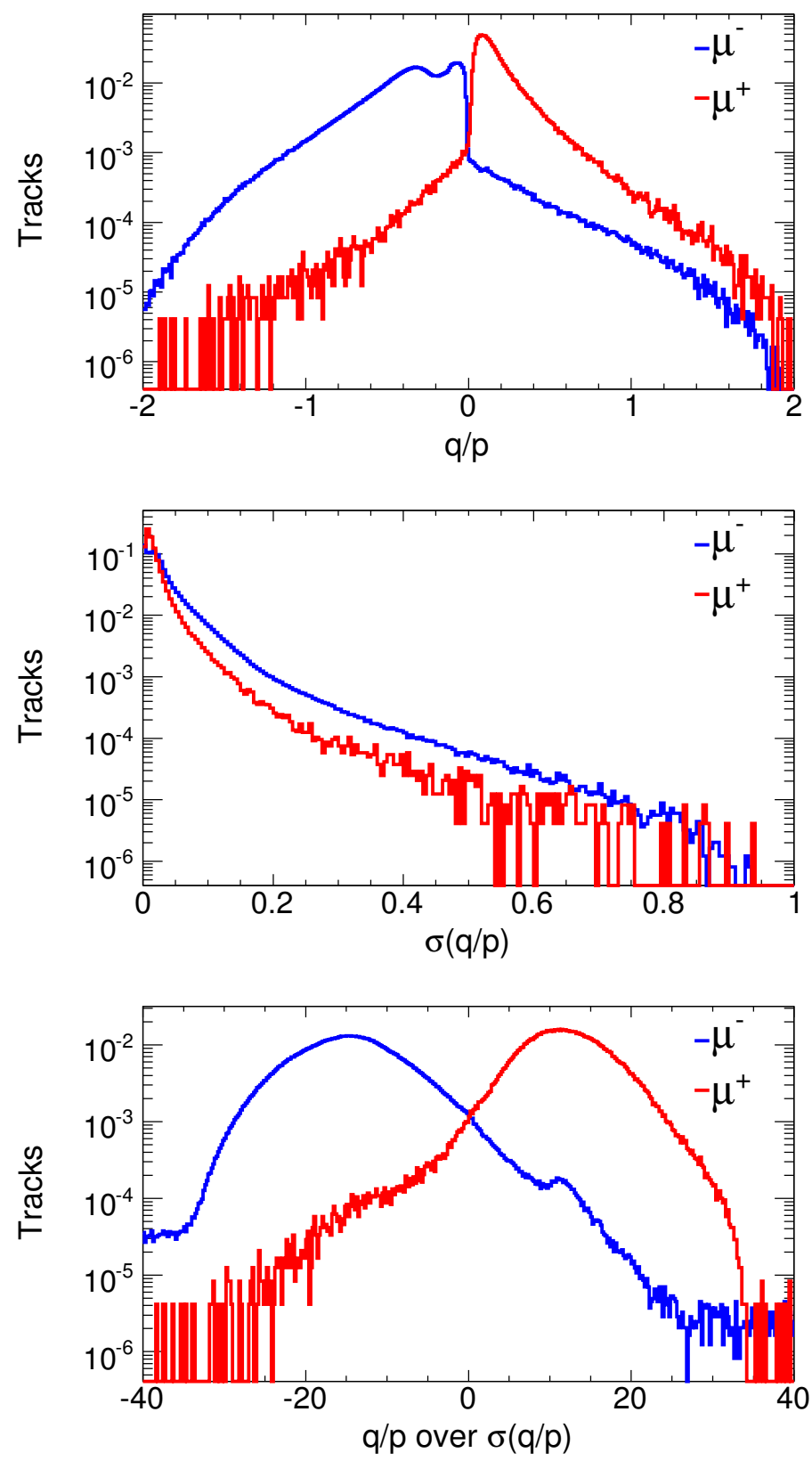

Figure 5.1: Variables computed by the track fitting algorithm are shown for the true $\mathrm{MC} \mu^{-}$and $\mu^{+}$tracks. The top figure shows $q / p$. The middle figure shows $\sigma_{q / p}$. The bottom figure shows $q / p$ divided by $\sigma_{q / p}$. The histograms are normalized to the unit area. 
flag. Only tracks that pass the track fitting algorithm are selected for the analysis. The distributions of $q / p, \sigma(q / p)$, and the ratio of $q / p$ over $\sigma_{q / p}$ are shown in Figure 5.1 for the true $\mu^{-}$and $\mu^{+}$tracks in the MC simulation of the near detector. The fitting algorithm correctly determines the muon charge sign for a majority of reconstructed tracks. In Figure 5.1, the histograms tails that cross zero represent tracks with an incorrectly measured muon charge sign.

\subsection{New method for charge-sign measurement}

The selection of the $\bar{\nu}_{\mu}$ charged-current events requires an efficient measurement of the muon charge sign. The fitter-based measurement of the muon charge sign for $\mu^{+}$and $\mu^{-}$tracks produces many tracks with an incorrectly measured charge sign. Thus, an improvement in the measurement of the muon charge sign is necessary. In this section, an improved method for the measurement of the muon charge sign is described.

In the MINOS detectors, the magnetic field lines follow concentric, nearly circular loops around a coil. Figure 5.2 shows the magnetic maps; the maps show field vectors tangent to the magnetic field lines. The direction of the magnetic field lines depends on the polarity of the coil current. There are two polarity settings: forward and reverse. Our analysis uses the data acquired with the forward coil polarity. For this polarity, a negatively charged particle traveling along the detector Z-axis experiences a Lorentz force directed toward the coil, and a positively charged particle is deflected away from the 

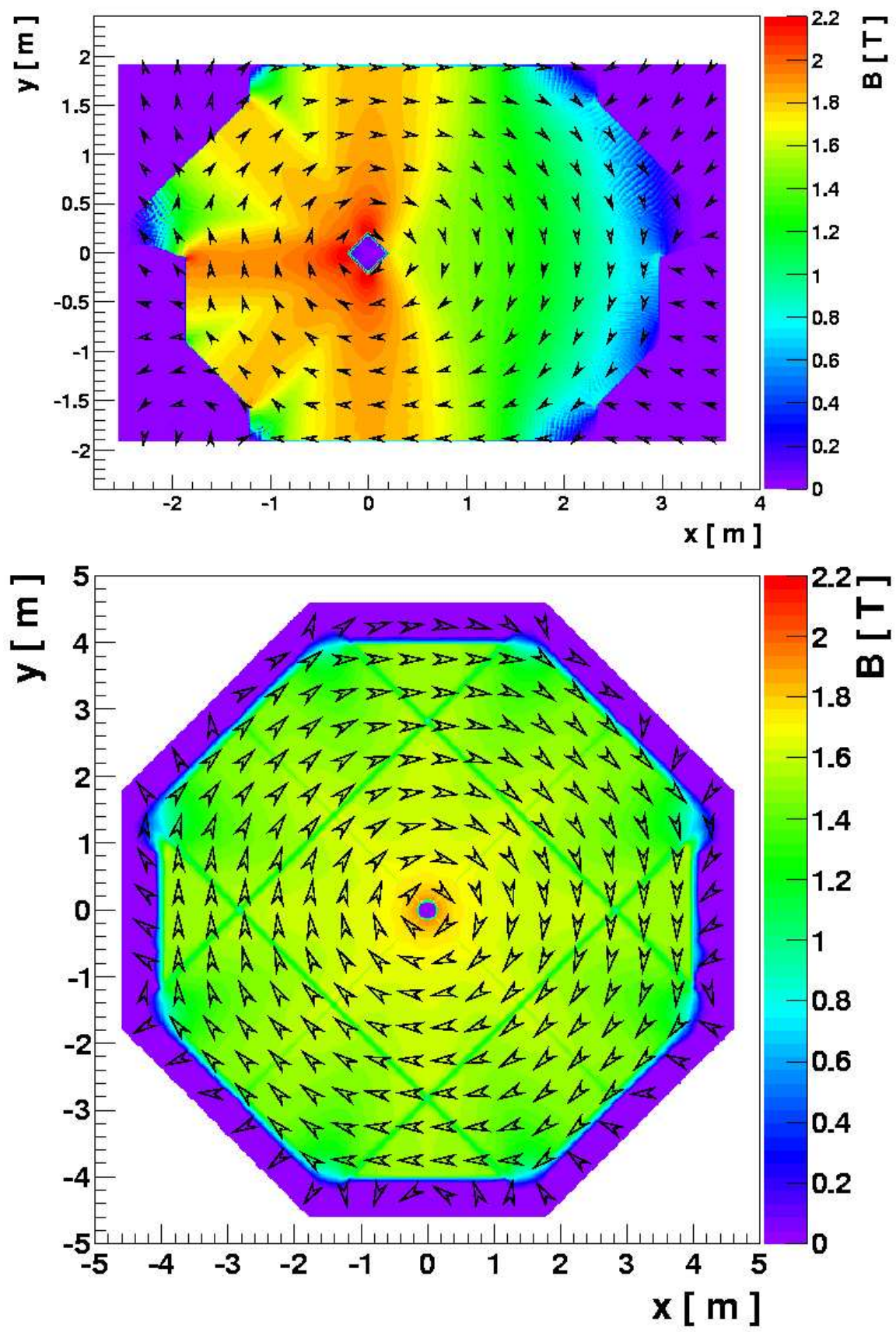

Figure 5.2: Near detector (top) and far detector (bottom) magnetic field maps. The two detectors have toroidal magnetic field geometry with an average field strength of around 1.2 T. Black arrows indicate the direction of the magnetic field lines. Figures are courtesy of Robert Hatcher. 
coil. Figure 5.3 shows the positions of muon track ends for the true $\mathrm{MC} \mu^{-}$ and $\mu^{+}$tracks. The majority of the $\mu^{-}$tracks move toward the coil, where many of these tracks stop. The majority of the $\mu^{+}$tracks move away from the coil and exit the detector.

The magnetic field induces a systematic deflection in the trajectory of a muon traveling through the MINOS detector. Our method computes a difference between the actual muon trajectory and the trajectory the muon would follow in an empty space (without matter and a magnetic field). This method exploits the toroidal geometry of the MINOS magnetic field and uses the direction of the muon momentum at the track vertex and the position of the track end. The muon direction at the track vertex is determined by the track fitting algorithm. ${ }^{1}$ The track vertex position, $V$, is projected along this muon direction to the point, $P$, in the last scintillator plane that contains the last track hit, E, as illustrated in Figure 5.4a. A right-handed Cartesian 2d coordinate system is constructed in the last scintillator plane, as illustrated in Figure 5.4b. The center of this coordinate system is at the projected point $P$; the $x$-axis is directed radially outward; and, the $y$-axis is orthogonal to the $x$-axis. In this coordinate system, $\mu^{-}$tracks bend toward the coil (toward the negative $x$ values), while $\mu^{+}$tracks bend away from the coil (toward the positive $x$ values).

Four interrelated variables are constructed using the geometry described

\footnotetext{
${ }^{1}$ If a track fails the fitting algorithm, then the muon direction is determined by the track finding algorithm; these tracks are not used for our analysis.
} 

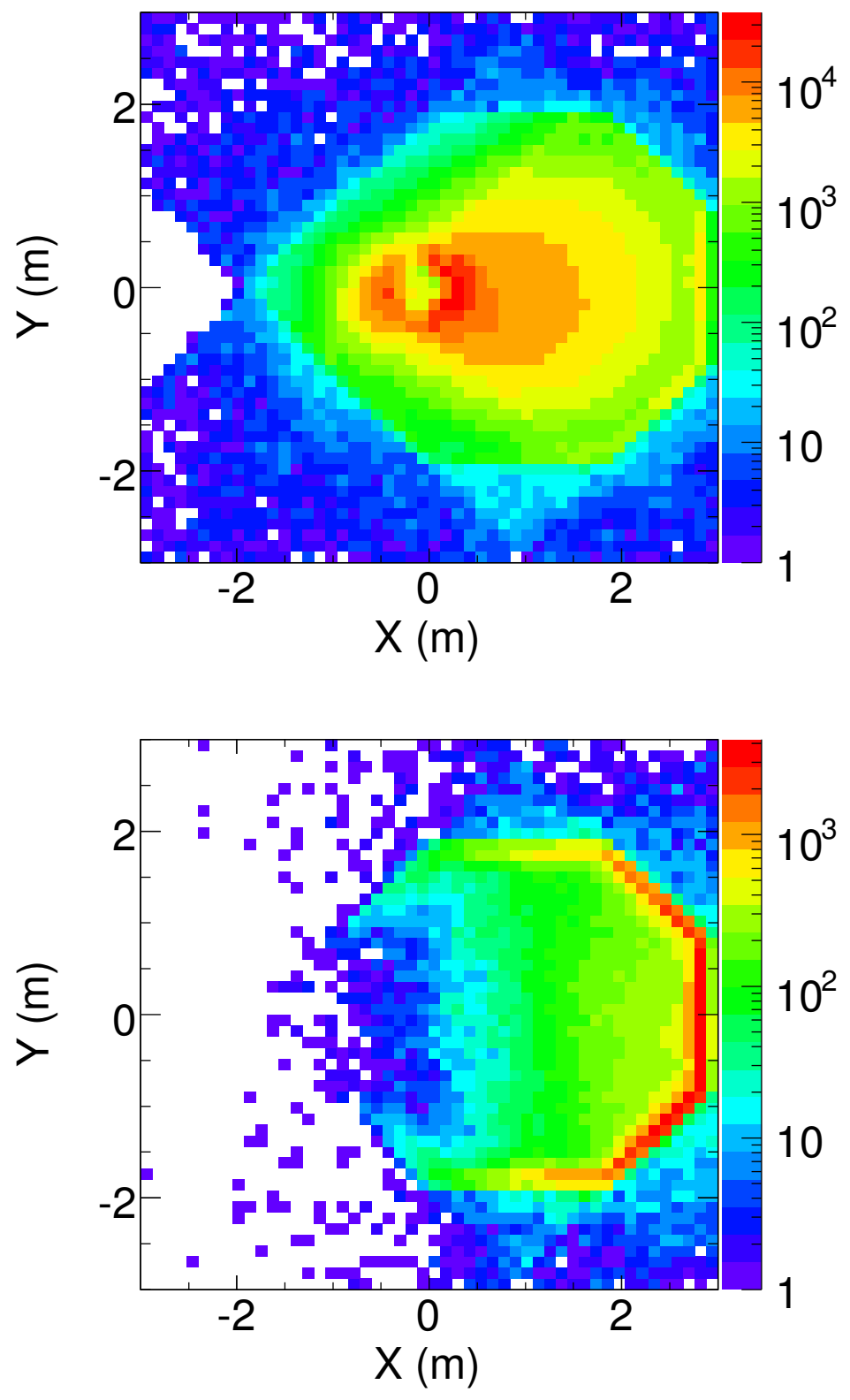

Figure 5.3: Figures show $y$ and $x$ track-end positions for the true $\mu^{-}$tracks (left) and $\mu^{+}$tracks (right) for the MC simulation of the near detector. 


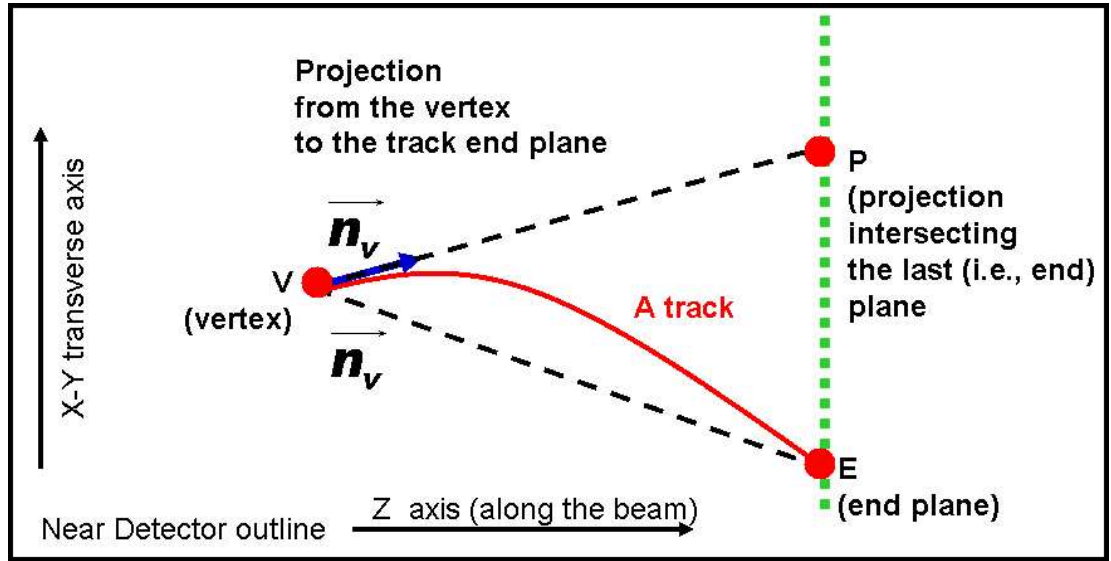

(a)

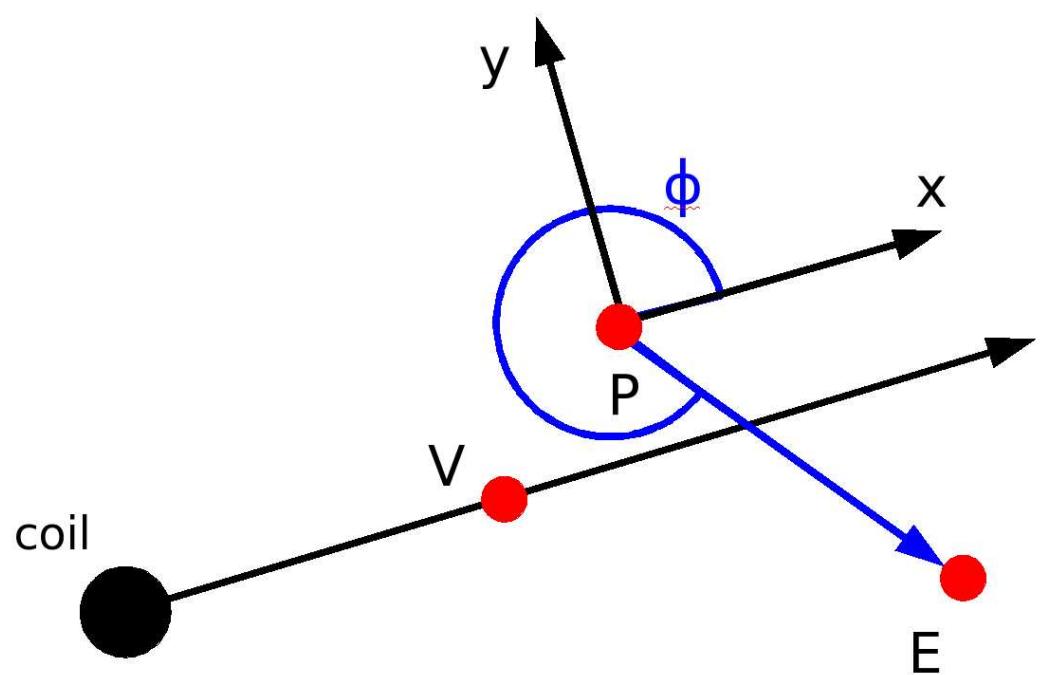

(b)

Figure 5.4: (a) Illustration of the charge-sign technique along the detector $\mathrm{Z}$ axis. A straight line along the initial track direction at the vertex, $\mathbf{n}_{\mathbf{V}}$, is used to project the vertex point $\mathbf{V}$ to the point $\mathbf{P}$ in the last track plane. The actual track ends at the point $\mathbf{E}$. (b) In the last track plane, the position of the last track hit $\mathbf{E}$ is shown; also shown are the projected point $\mathbf{P}$, and the vertex point V. A right-handed Cartesian 2d coordinate system is constructed such that the $x$-axis points radially outward (parallel to the line that passes through the coil and the track vertex), and the $y$-axis is orthogonal to the $x$-axis. The center of this coordinate system is at the point $\mathbf{P}$. 

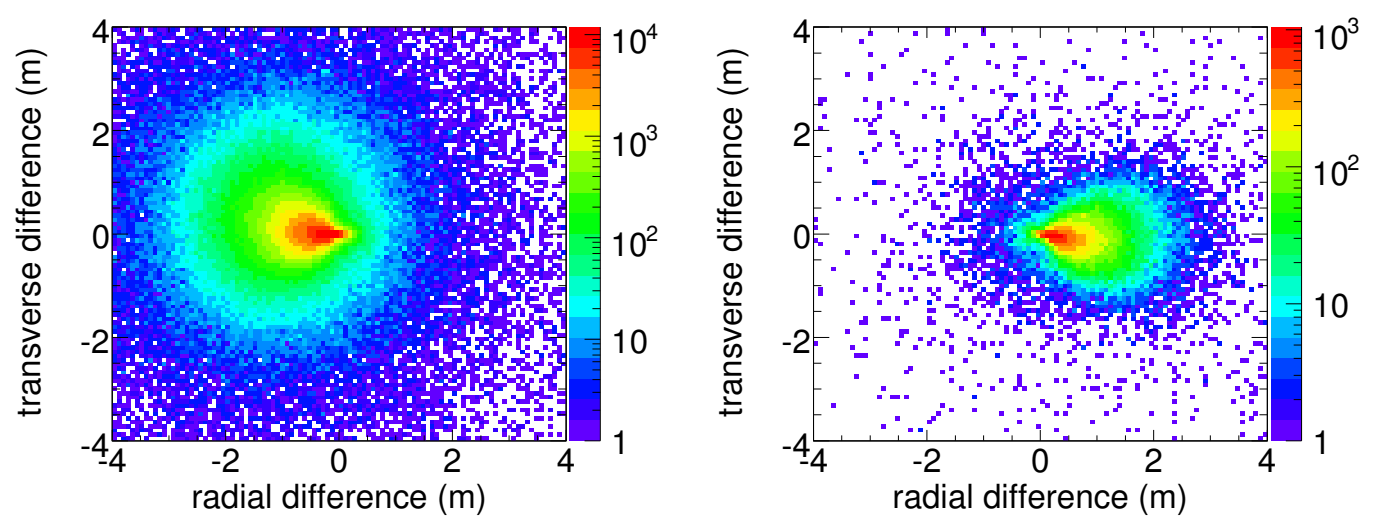

Figure 5.5: Differences between actual and projected muon trajectories. The figures show positions of the point $\mathbf{E}$ relative the point $\mathbf{P}$, using the coordinate system defined in Figure 5.4b. The left figure shows the $\mu^{-}$tracks, and the right figure shows $\mu^{+}$tracks using the MC simulation of the near detector.
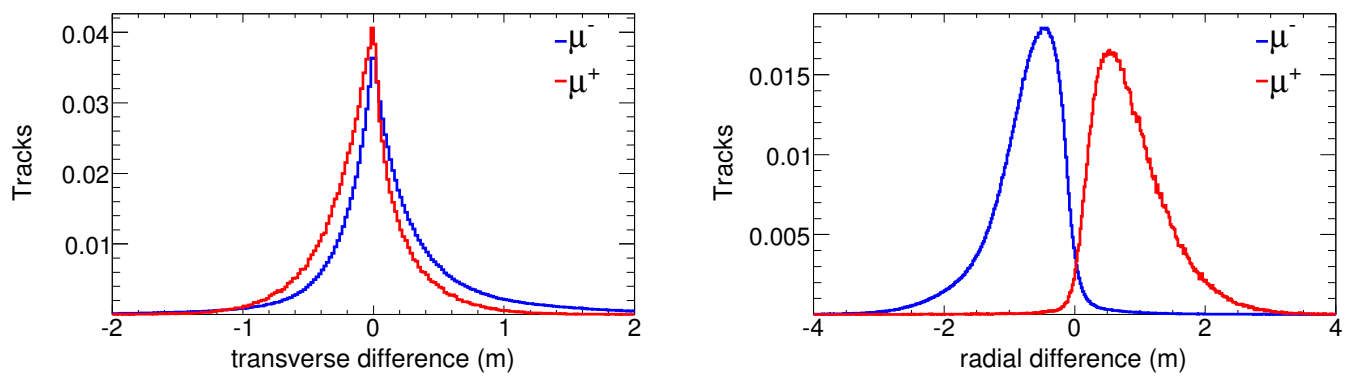

Figure 5.6: Projections onto the $y$ axis (left) and $x$ axis (right) of the scatter plots in Figure 5.5. These figures show the projections of the distance PE onto the $x$-axis and $y$-axis, defined in Figure 5.4b. The histograms are normalized to the unit area. The figures show the $\mu^{-}$and $\mu^{+}$tracks from the MC simulation of the near detector. 

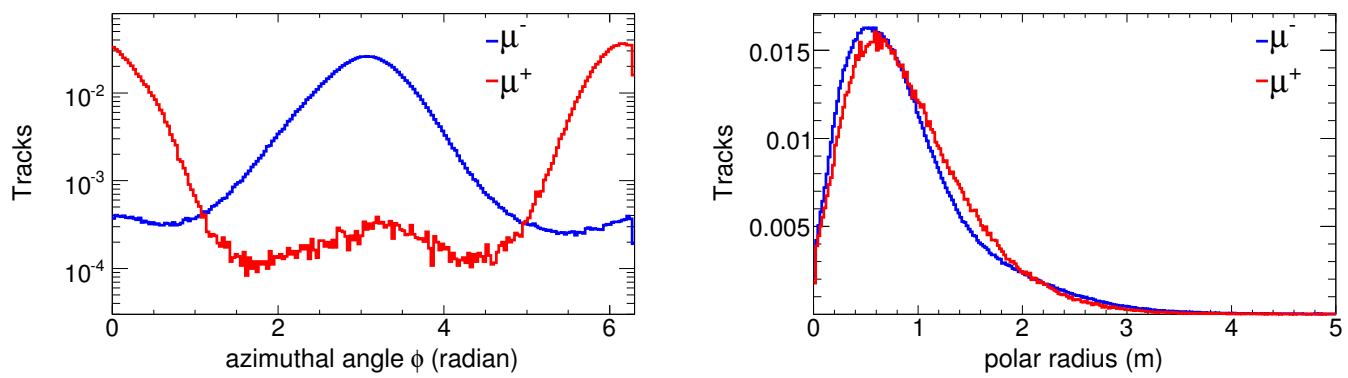

Figure 5.7: Figures show the azimuthal angle (left) and the polar radius PE (right), defined in Figure 5.4b. The histograms are normalized to the unit area. The figures show $\mu^{-}$and $\mu^{+}$tracks from the $\mathrm{MC}$ simulation of the near detector.

above. These variables measure the amount and the sign of the muon track deflection in the MINOS magnetic field. First, "radial difference" and "transverse difference" variables are defined. Using the notation in Figure 5.4b, the radial difference is $\Delta x=E_{x}-P_{x}$, and the transverse difference is $\Delta y=E_{y}-P_{y}$. These two variables are plotted in Figure 5.6. The radial difference depends on the muon charge sign, the muon momentum, and the strength of the magnetic field; this variable measures the muon deflection perpendicular to the magnetic field lines. The transverse difference measures the muon deflection parallel to the magnetic field lines. Because a charged particle traveling parallel to the magnetic field lines experiences no Lorentz force, the transverse difference is mostly independent of the magnetic field. This variable strongly depends on the multiple Coulomb scattering of muons in the MINOS detectors.

The last two variables are an azimuthal angle $\phi$ and a polar radius, $\mathbf{P E}$; these variables are the polar coordinates of the point $\mathbf{E}$ in the $2 \mathrm{~d}$ coordinate 

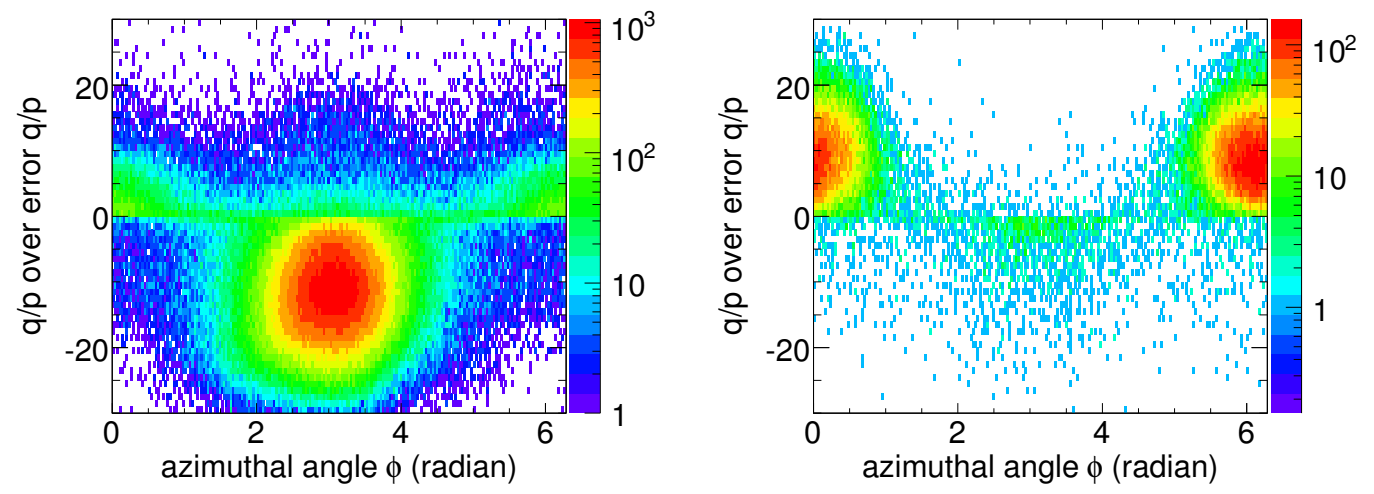

Figure 5.8: Azimuthal angle $\phi$ (defined in the text) plotted versus the ratio of $\frac{q / p}{\sigma_{q / p}}$ for the $\mu^{-}$(left) and $\mu^{+}$(right) tracks from the MC simulation of the near detector.

system defined in Figure 5.4b. Figure 5.7 shows the distributions of these two variables. The azimuthal angle is strongly correlated with the muon charge sign. Figure 5.7 shows the separation between the $\mu^{-}$and $\mu^{+}$tracks. Figure 5.8 shows correlations between the azimuthal angle $\phi$ and the ratio $\frac{q / p}{\sigma_{q / p}}$ for the $\mu^{-}$and $\mu^{+}$tracks. The azimuthal angle $\phi$ is a new variable used for the measurement of the muon charge sign.

\subsection{Charge-sign for non-muon tracks}

It is interesting to note that the curvature of the non-muon tracks does not depend on the magnetic field. Figure 5.9 shows the azimuthal angle $\phi$ and the polar radius for the muon and non-muon tracks, and Figure 5.9 shows these variables for the $\pi^{-}$and $\pi^{+}$tracks. The non-muon tracks do not experience significant deflections in the magnetic field. For these tracks, the 
deflections are independent of the charge sign. These observations suggest that the MINOS magnetic field has a negligible effect on the non-muon tracks. Figure 4.4 shows a number of track planes for the non-muon tracks; these tracks are typically very short and do not have a sufficient number of hits to measure the track curvature. More importantly, many of these tracks are found in hadronic showers and thus do not have a well defined track curvature.

\subsection{Selecting muon neutrino and anti-neutrino events}

In this section a new charge-sign method is examined using the $\mathrm{MC}$ simulation. First, events are selected using the muon discriminant variable $P_{S}$, defined in Equation 4.5. The events with $P_{S}>0.3$ are identified as either $\nu_{\mu}$ charged-current or $\bar{\nu}_{\mu}$ charged-current candidate events. The $\nu_{\mu}$ chargedcurrent and $\bar{\nu}_{\mu}$ charged-current events are separated using the charge sign of the reconstructed tracks. The longest track in the event is used to measure the muon charge sign. The standard (default) charge-sign selection uses the $q / p$ variable computed by the track fitting algorithm for the reconstructed track in the event. The events with $q / p<0$ are identified as the candidate $\nu_{\mu}$ chargedcurrent events. The events with $q / p>0$ are identified as the candidate $\bar{\nu}_{\mu}$ charged-current events.

Figure 5.10 shows the new sign charge-sign variable (azimuthal angle $\phi$ ) for the tracks that pass the default charge-sign selection. Figure 5.10a shows the azimuthal angle $\phi$ for the true $\mu^{+}$and $\mu^{-}$tracks with $q / p<0$; there is no significant background contamination from the true $\mu^{+}$tracks. Figure 5.10b 


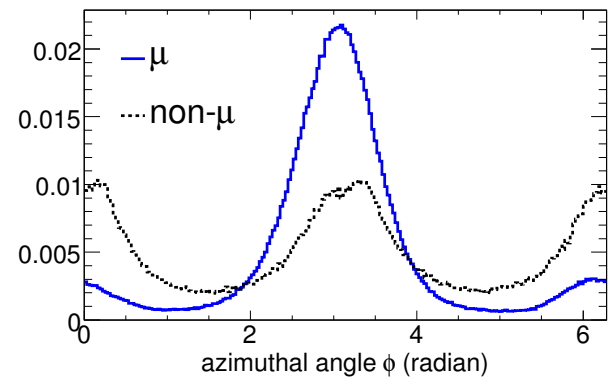

(a)

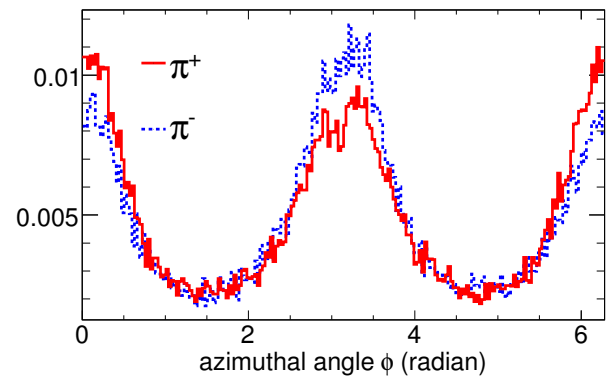

(c)

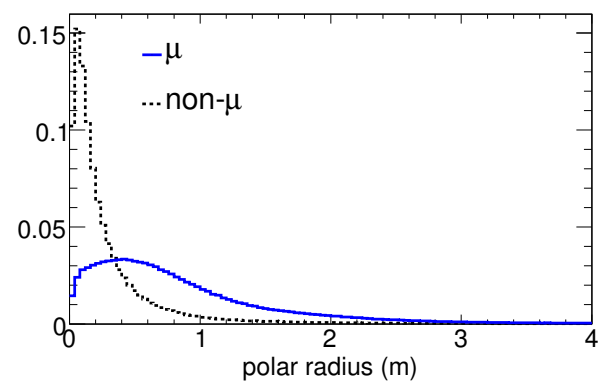

(b)

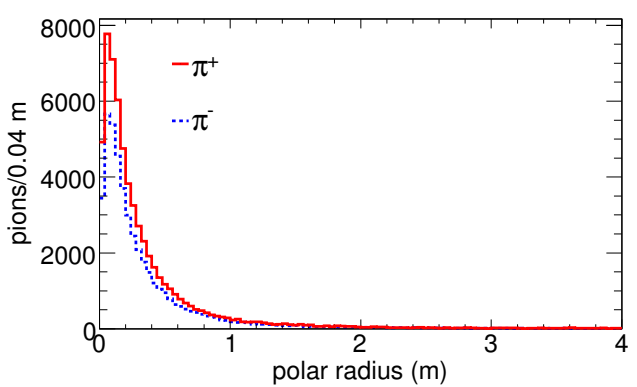

(d)

Figure 5.9: Azimuthal angle $\phi$ and polar radius. These variables are defined in Figure 5.4b. Figures (a) and (b) show the muon and non-muon tracks. Figures (c) and (d) show the $\pi^{-}$and $\pi^{+}$tracks. The histograms are normalized to the unit area (except for Figure $(\mathrm{d})$ ). The figures are made using the MC simulation of the near detector. 


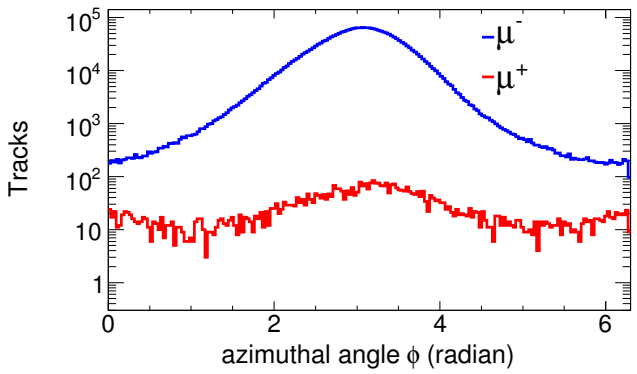

(a)

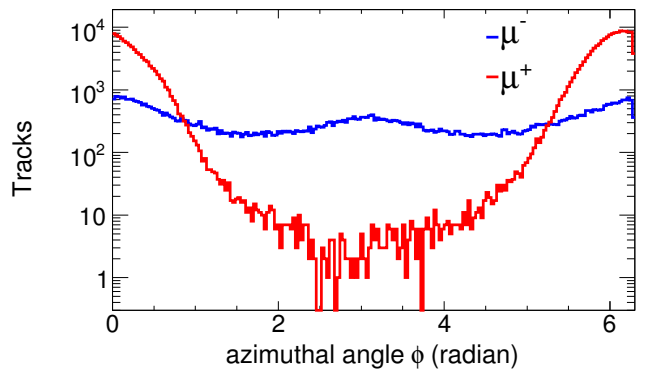

(b)

Figure 5.10: Azimuthal angle $\phi$, the new charge-sign variable defined in Figure $5.4 \mathrm{~b}$, plotted after the standard (default) charge-sign selection for the $\mu^{+}$ and $\mu^{-}$tracks. Figure (a) shows the azimuthal angle for the reconstructed tracks with $q / p<0$. Figure (b) shows the azimuthal angle for the reconstructed tracks with $q / p>0$

shows the azimuthal angle $\phi$ for the true $\mu^{+}$and $\mu^{-}$tracks with $q / p>0$; there is a significant number of the $\mu^{-}$background tracks.

The new charge-sign method is defined as follows:

- The positive charge-sign track selection: $\frac{q / p}{\sigma(q / p)}>2, \phi<1$ or $\phi>5$,

- The negative charge-sign track selection: $\frac{q / p}{\sigma(q / p)}<-2, \phi>1$ and $\phi<5$.

The $\frac{q / p}{\sigma(q / p)}<-2$ requirement is more stringent than the usual requirement of $q / p<0$. It was implemented to remove tracks with errors measuring the $q / p$ value.

The efficiency of the charge-sign measurement depends on the number of the scintillator hits in a reconstructed track. Longer tracks measure the track curvature at many points, which improves the efficiency of the curvature 


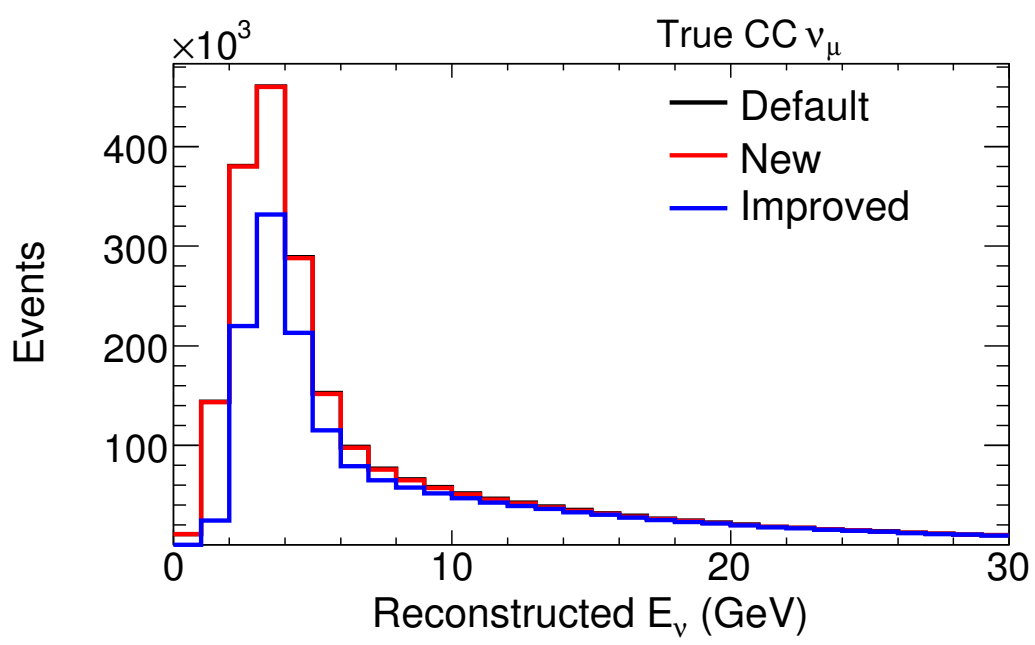

(a)

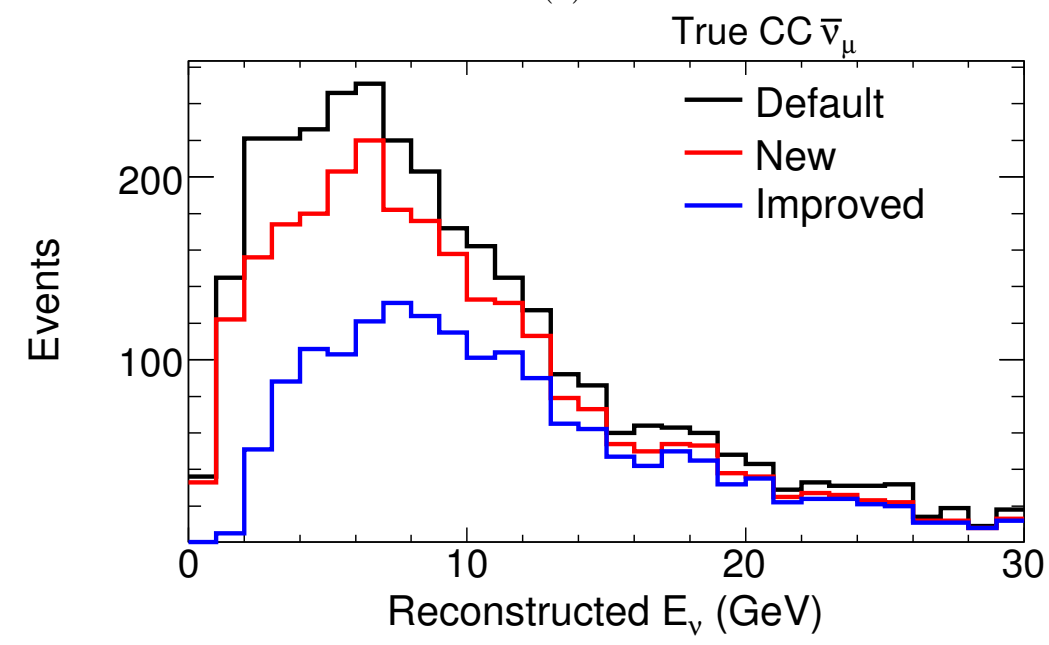

(b)

Figure 5.11: Reconstructed energy for the near detector events that pass one of three negative charge-sign selections described in the text: default, new, and improved. Figure (a) shows the true $\nu_{\mu}$ charged-current interactions. Figure (b) shows the true $\bar{\nu}_{\mu}$ charged-current interactions. 


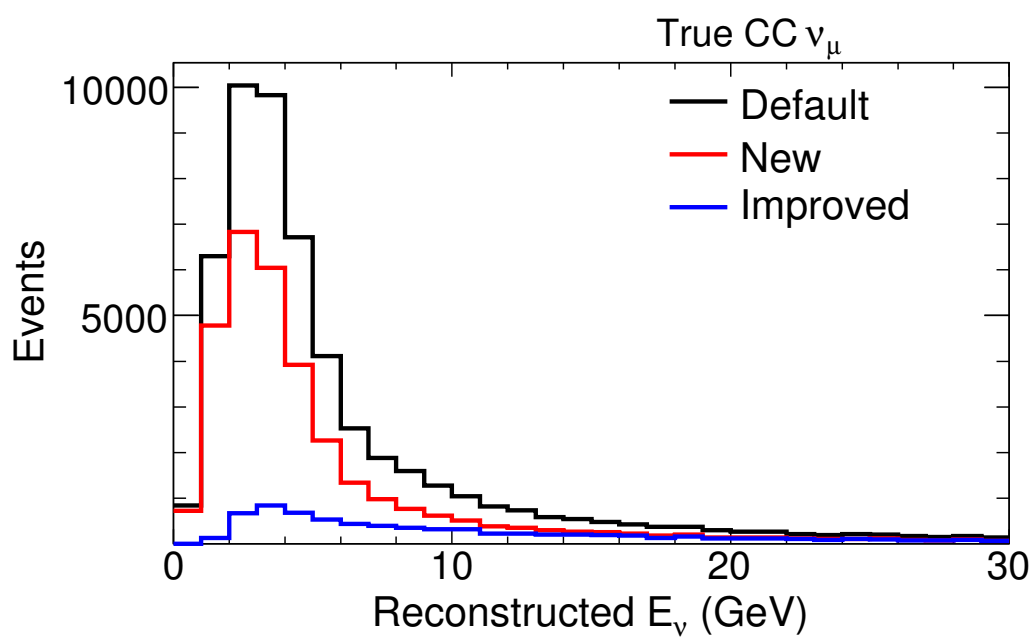

(a)

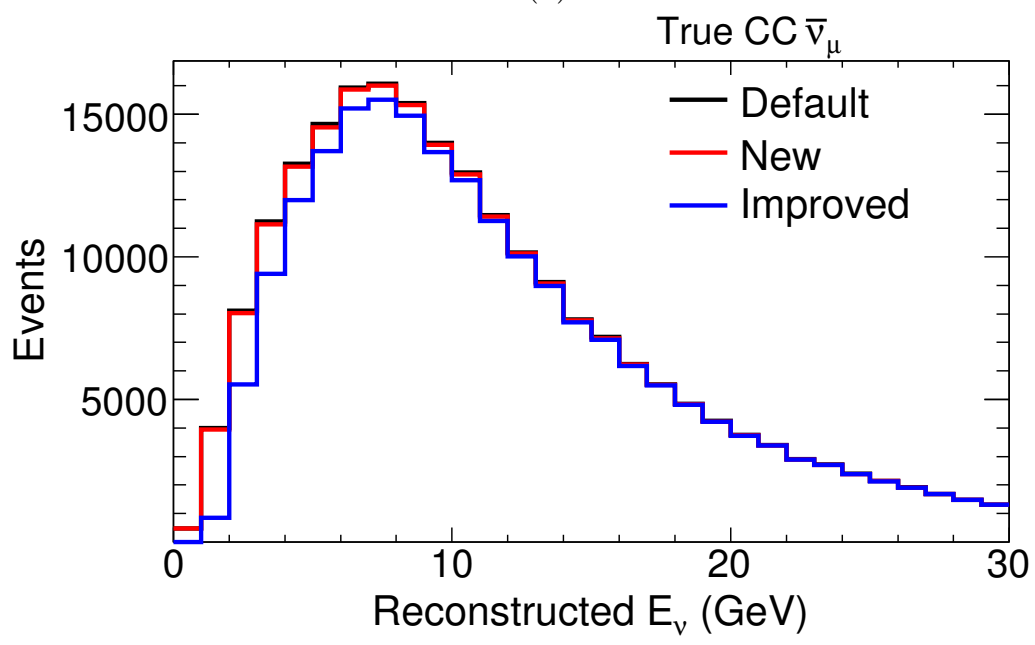

(b)

Figure 5.12: Reconstructed energy for the near detector events that pass one of three positive charge-sign selections described in the text: default, new, and improved. Figure (a) shows the true $\nu_{\mu}$ charged-current interactions. Figure (b) shows the true $\bar{\nu}_{\mu}$ charged-current interactions. 
measurement. The new charge-sign selection is improved by requiring that the tracks contain at least 20 hits in both detector views (the improved charge-sign method).

Figure 5.11 compares the reconstructed energy spectra for the candidate $\nu_{\mu}$ charged-current events selected with the default, new, and improved methods. Figure 5.11a shows the true $\nu_{\mu}$ charged-current events. There is no significant change in the number of the selected events between the default and new methods. Figure 5.11b shows the true $\bar{\nu}_{\mu}$ charged-current events. There is a small reduction in the number of these background events. The conclusion is that the new charge-sign method does not affect the selection of the $\nu_{\mu}$ charged-current events.

Table 5.1 lists the number of $\nu_{\mu}$ charged-current events and $\bar{\nu}_{\mu}$ chargedcurrent events that pass one of the three positive charge-sign methods: default, new, and improved. The fraction of the $\nu_{\mu}$ charged-current events (background) is significantly reduced with the new and improved charge-sign methods.

Figure 5.12 compares the reconstructed energy spectra for the candidate $\bar{\nu}_{\mu}$ charged-current events selected with the default, new, and improved methods. Figure 5.12a shows the true $\nu_{\mu}$ charged-current events. The new and improved methods remove a large fraction of the background $\nu_{\mu}$ chargedcurrent events, as summarized in Table 5.1. Figure 5.12b shows the true $\bar{\nu}_{\mu}$ charged-current events. There is no noticeable reduction in the number of true $\bar{\nu}_{\mu}$ charged-current events between the default and new selections. 


\begin{tabular}{l|rrr} 
& Default & New & Improved \\
\hline$N_{\bar{\nu}_{\mu}}$ & 228880 & 227598 & 215071 \\
\hline$N_{\nu_{\mu}}$ & 55460 & 33550 & 8535 \\
\hline $100 \% \times N_{\bar{\nu}_{\mu}} /\left(N_{\bar{\nu}_{\mu}}+N_{\nu_{\mu}}\right)$ & $24.23 \%$ & $14.74 \%$ & $3.97 \%$
\end{tabular}

Table 5.1: Number of true $\bar{\nu}_{\mu}$ charged-current and $\nu_{\mu}$ charged-current events among the selected events with the positive charge sign, as computed by the three methods. The new and improved charge sign methods significantly reduce a number of the background $\nu_{\mu}$ charged-current events.

\subsection{Summary}

In this chapter, a new and improved method for the measurement of the muon charge sign in the MINOS detectors was introduced. The method exploits the toroidal geometry of the magnetic field in the MINOS detectors. The method is used in combination with the standard charge-sign method to select the $\bar{\nu}_{\mu}$ charged-current candidate events. This selection can be further improved by requiring that the tracks contain at least 20 hits in each detector view. For this improved selection, the number of $\nu_{\mu}$ charged-current background events is reduced from $24.2 \%$ to $3.97 \%$. 


\section{Chapter 6}

\section{Muon neutrino events in the near detector}

The main purpose of the MINOS near detector is to measure properties of neutrino interactions produced by the neutrino beam, before neutrino oscillation occur. This measurement is then used to compute an expected number of $\nu_{\mu}$ charged-current events at the far detector. A study of the $\nu_{\mu}$ chargedcurrent events in the near detector events also improves our understanding of the neutrino physics and the characteristics of the near detector. The MINOS detectors are built to be similar, so an improved knowledge of the near detector directly transfers to the far detector.

Section 6.1 describes the calibration of the near detector magnetic field. This procedure uses the muon tracks, produced by neutrino interactions, that stop within the near detector. The goal of this work is to understand a relationship between the curvature of muon tracks and the near detector magnetic field. The MC simulation is employed to relate the muon momentum from the curvature to the more precise measurement of the muon momentum from the range. This work resulted in an improved understanding of the magnetic field in the MINOS detectors and reduced the systematic error for the measurement of the muon momentum from the curvature. 
In this chapter, the near detector events from several beam configurations are examined independently. Initial observations show substantial disagreements between the data and (default) MC simulation. The largest disagreements are attributed to the uncertainty of computing the pion production rate from the interaction of $120 \mathrm{GeV} / \mathrm{c}$ protons with the NuMI target [58]. The goal of our analysis is twofold. First, the previous flux analysis [101] is extended to measure the quasielastic scattering (QES) and resonance production (RES) event rates in the near detector. This work is discussed in Chapter 7. Second, these near detector measurements allow for an oscillation analysis of the QES and RES events separate from the deep inelastic scattering (DIS) events. This oscillation analysis is presented in Chapter 9.

In Section 6.2, the event selection requirements from the earlier chapters are summarized. In Section 6.3, the $\nu_{\mu}$ charged-current events in the near detector are examined. These events are compared with two versions of the MC simulation: default and tuned. The tuned MC simulation is derived using a tuning procedure described in Chapter 7. Briefly, the tuning procedure computes corrections for the neutrino flux mode and the cross-sections model; these corrections are computed by minimizing differences between the data and $\mathrm{MC}$ events.

\subsection{Calibration of the near detector magnetic field}

The near detector magnetic field is calibrated using muon tracks. These muons are produced when beam neutrinos interact with the near detector; 
a large number of these muons supply ample statistics for this calibration task. First, the $\mu^{-}$tracks are selected using the muon identification method described in Chapter 4 and the (improved) charge-sign method described in Chapter 5. The long muons are selected by requiring that the muon tracks contain at least 20 planes in each of the two detector views.

A fraction of the muons in the 1-6 GeV momentum range stops within the near detector. For the stopping muons, two independent measurements of the muon momentum are available. First, the momentum of these tracks is measured via range by integrating the track range through the detector material and using range tables [75]. The uncertainty of this measurement is $2 \%$, which is derived based on the uncertainty of the MC geometry simulation, the uncertainty of the detector mass, and the uncertainty of the track finding. Second, the muon momentum is measured via the curvature using a track fitting algorithm. ${ }^{1}$ This algorithm, called the alternative algorithm, is indepedent from the track fitting algorithm discussed in Section 3.1 (the standard algorithm). Figure 6.1 shows the muon momentum measured via the range and the curvature using the standard algorithm. The alternative fitting algorithm provides a measurement of the track curvature that does not depend on the track range. This algorithm employs an iterative procedure to minimize the differences between actual track hits and the parametrized muon trajectory through the detector. The procedure takes into account effects from

\footnotetext{
${ }^{1}$ The track fitting algorithm used in this section was developed by Sergei Avvakumov and it is based on techniques described in [102].
} 

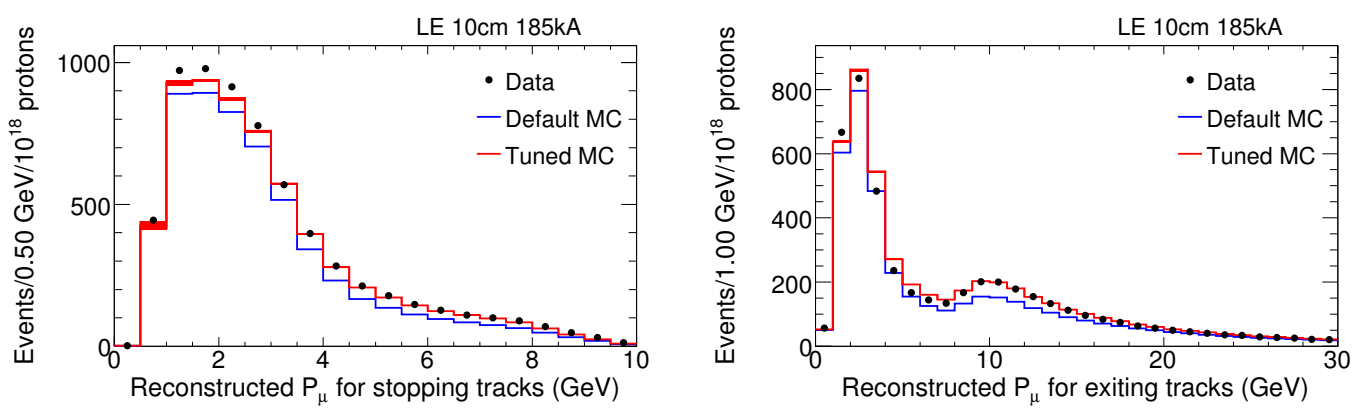

Figure 6.1: The left figure shows the muon momentum measured via range, for the tracks that stop within the near detector detector. The right figure shows the muon momentum measured via curvature, for the tracks that exit the near detector detector. These figures show the near detector events for the data and two versions of the MC simulation, described in Section 6.3. The $\mathrm{MC}$ histograms are scaled to the number of POT in the data.

the multiple Coulomb scattering, muon energy loss in the detector material, and bending in the magnetic field [102].

A comparison of the muon momentum from the range, $P_{\text {range }}$, with the muon momentum from the curvature, $P_{\text {curv }}$, provides a tool for the calibration of the magnetic field strength in the near detector. This calibration approach provides an overall magnetic field calibration factor. The calibration procedure compares the $P_{\text {range }} / P_{\text {curv }}$ ratio for the data tracks and the $\mathrm{MC}$ tracks. In the MC simulation, the magnetic field map is used for the simulation of muon propagation through the detector. The momentum from the range, $P_{\text {range }}$, for the data and MC simulation is known to within $2 \%$. Differences between the data and $\mathrm{MC}$ simulation for the $P_{\text {range }} / P_{\text {curv }}$ ratio greater than $2 \%$ are attributed to an incorrect scale of the near detector magnetic field.

The magnetic field maps for the near and far detectors are obtained 
in a finite element analysis [52]. Briefly, the analysis uses the "B-H" curve magnetization data obtained using test toruses manufactured from the actual near and far detector steel. Initial magnetic maps were computed using a limited sample of the detector steel; these maps are called "old" magnetic field maps. A subsequent analysis used an expanded test sample and additional detector data collected in-situ. The maps produced with this analysis are called "new" magnetic field maps, shown in Figure 5.2. The new magnetic field maps are used for our analysis of the near and far detector events. The old magnetic field maps are only used for the analysis in this section.

A study was performed to establish a relationship between the strength of the near detector magnetic field and the muon momentum from the curvature. For this study, three versions of the old magnetic field map were employed to reconstruct the muon momentum from the curvature for the data muon tracks. The old magnetic field map was scaled uniformly throughout the near detector by the following three scale factors: $B_{\text {scale }}=1,1.08,1.13$. In Figure 6.5, the $P_{\text {range }} / P_{\text {curv }}$ ratio is plotted as a function of the scale of the old magnetic field. The following relationship between the $P_{\text {range }} / P_{\text {curv }}$ ratio and the magnetic field scale was obtained:

$$
\frac{P_{\text {range }}}{P_{\text {curv }}}=1.413-0.419 \times B_{\text {scale }}
$$

Figures 6.3 shows the $P_{\text {range }} / P_{\text {curv }}$ ratio for the near detector muon tracks using three magnetic field maps, as explained in the figure caption. For the standard fitting algorithm, a significant correlation between $P_{\text {range }}$ 


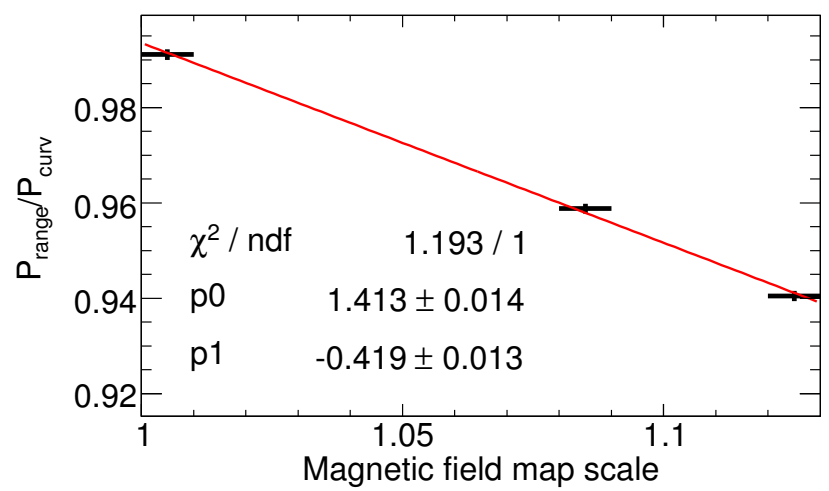

Figure 6.2: Mean of the ratio of the muon momentum from range over the momentum from the curvature plotted a function of the magnetic field scale, for the three scale factors: $1,1.08$, and 1.13. The mean is determined using Gaussian fit, similarly to the fit shown in Figure 6.5. The near detector muon tracks were used to make this figure.

and $P_{\text {curv }}$ is present in the computation of the muon momentum from the curvature. This bias improves the resolution of the muon momentum from the curvature. There is a few percent difference between the data and MC simulation. However, because of the correlation between $P_{\text {range }}$ and $P_{\text {curv }}$, it is difficult to draw any conclusions about the strength of the magnetic field in the near detector using the standard fitting algorithm.

Figures 6.4 shows the $P_{\text {range }} / P_{\text {curv }}$ ratio for the muon tracks in the near detector data and $\mathrm{MC}$ simulation. In this figure, the muon momentum from the curvature is computed using the alternative fitting algorithm. For the old magnetic field map, there is an approximately $5 \%$ shift between the data and $\mathrm{MC}$ simulation. Assuming that the muon momentum from the range is accurately measured, this figure implies that the muon momentum from the 

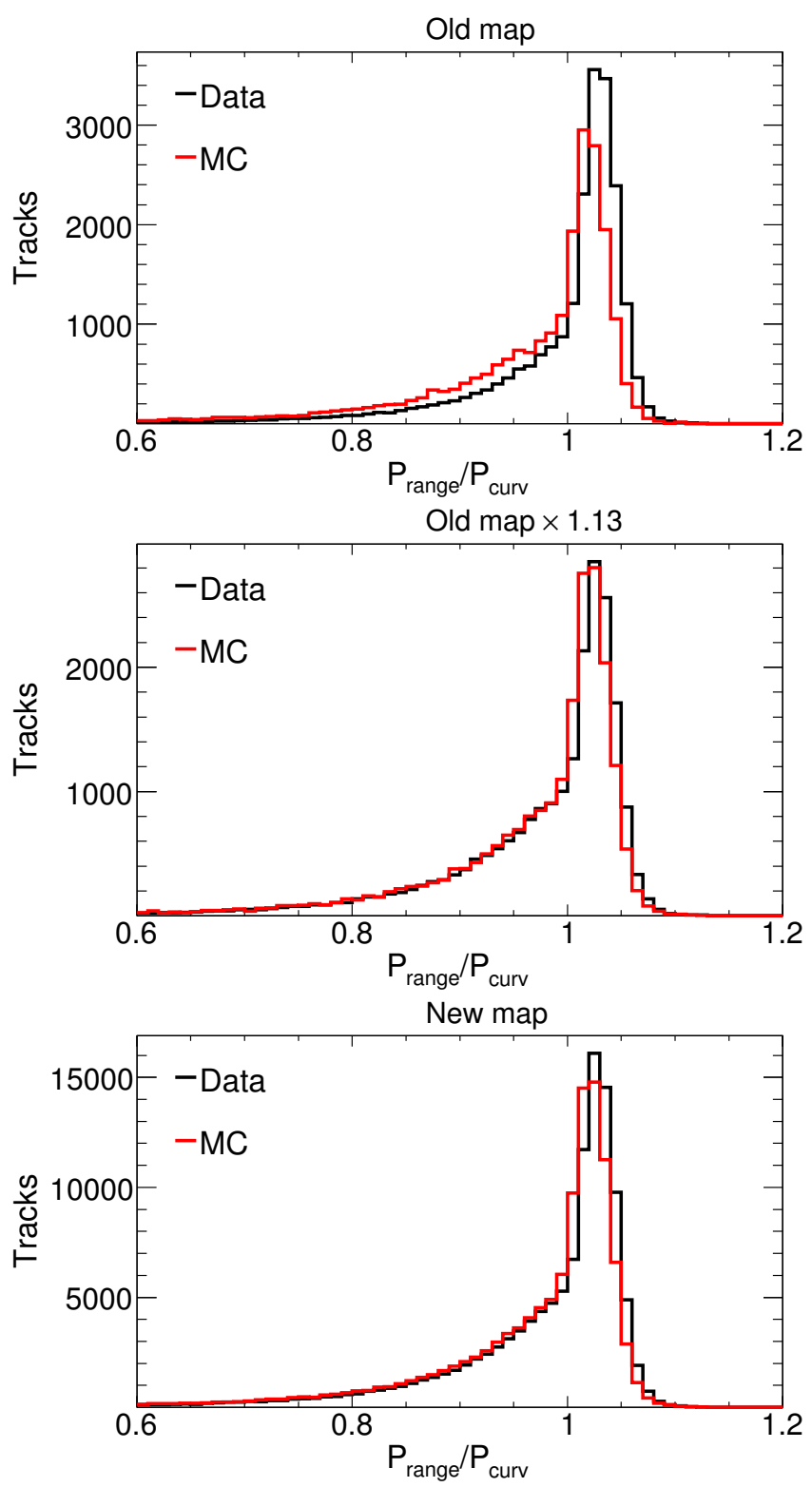

Figure 6.3: These figures show the ratio of the muon momentum from the range over the muon momentum from the curvature, $P_{\text {range }} / P_{\text {curv }}$ for the three magnetic field maps, as discussed in the text. The muon momentum from the curvature, $P_{\text {curv }}$, is computed using the standard fitting algorithm. These figures show the tracks for the near detector data and $\mathrm{MC}$ simulation. The number of the data tracks is scaled down to match the number of the MC tracks. 

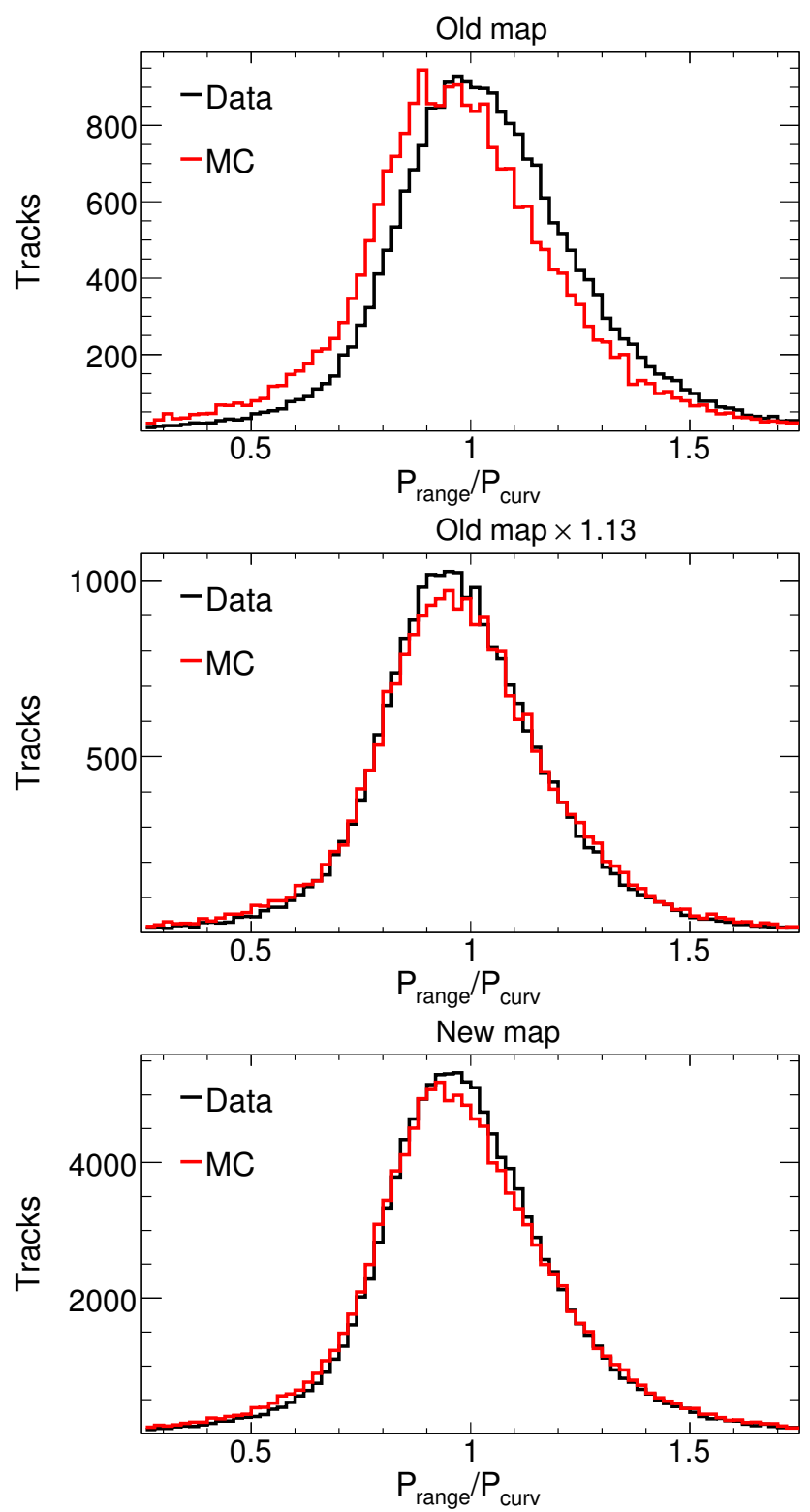

Figure 6.4: These figures show the ratio of the muon momentum from the range over the muon momentum from the curvature, $P_{\text {range }} / P_{\text {curv }}$ for the three magnetic field maps, as discussed in the text. The muon momentum from the curvature, $P_{\text {curv }}$, is computed using the alternative fitting algorithm. These figures show the tracks for the near detector data and MC simulation. The number of the data tracks is scaled down to match the number of the MC tracks. 


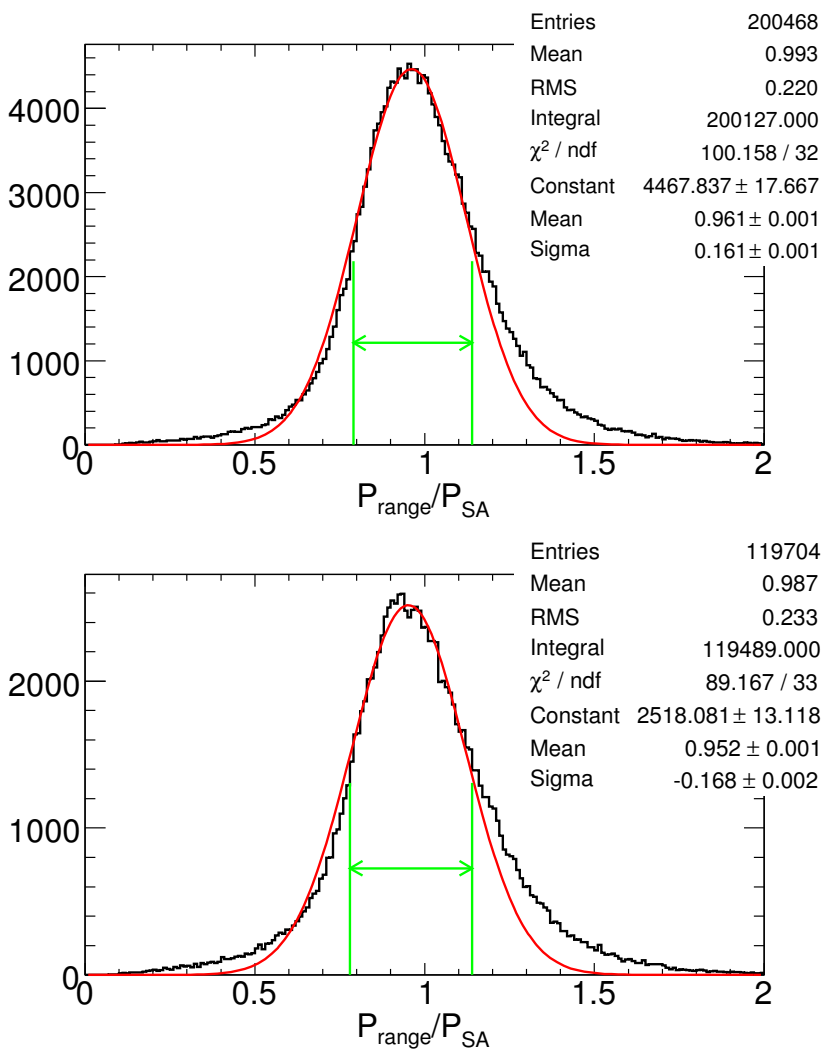

Figure 6.5: Ratio of the muon momentum from the range over the muon momentum from the curvature using the new magnetic field map for the data (top) and the MC (bottom) muon tracks in the near detector. The ratios are fitted with a Gaussian function within a range marked by green lines, located at $60 \%$ of the peak value. The difference between the Gaussian means for the data and the MC simulation is approximately $1 \%$. 
curvature is incorrect by approximately $5 \%$. This $5 \%$ shift translates into the $13 \%$ error on the scale of the near detector magnetic field. Equation 6.1 was used to relate the muon momentum from the curvature to the magnetic field stregnth. Figures 6.4 shows that the $P_{\text {range }} / P_{\text {curv }}$ ratio, for the tracks reconstructed with the 1.13 scaled magnetic field map, is the same in the data and the MC simulation. This observation prompted an investigation of the magnetic field properties in the near detector and far detectors; this work produced the new magnetic field maps, discussed earlier. The new magnetic maps are approximately $12.3 \%$ and $9.2 \%$ stronger than the old maps for the near and far detectors respectively (averaged over the detector fiducial volume). The difference for the $P_{\text {range }} / P_{\text {curv }}$ ratio between the data and the MC simulation with the new map is approximately 1\%, as shown in Figure 6.5.

An error on the muon momentum from the curvature is obtained from a comparison of the stopping muon tracks in the data and the MC simulation. Figure 6.5 shows the $P_{\text {range }} / P_{\text {curv }}$ ratio computed with the new magnetic field map. The difference between the peaks of the data and the MC distributions is approximately $1 \%$. This error is added linearly with the $2 \%$ uncertainty of the muon momentum from the range to produce the $3 \%$ uncertainty of the absolute muon momentum from the curvature. Because the near and far detectors use steel from the same foundry, the same error is used for the muons in the far detector. 


\subsection{Selecting near detector events}

The reconstructed events are required to contain at least one reconstructed track to be selected for our analysis. The track ${ }^{2}$ is required to originate within the fiducial volume of the near detector. The fiducial volume is a cylinder with a radius of $80 \mathrm{~cm}$, which spans 55 detector planes (steel planes 14 through 68$)$ :

$$
\begin{aligned}
& \sqrt{(X-1.4828)^{2}+(Y-0.2384)^{2}}<0.8 \mathrm{~m}, \\
& 0.81009 \mathrm{~m}<Z-0.0392 \mathrm{~m}<4.07710 \mathrm{~m}
\end{aligned}
$$

where $X, Y$, and $Z$ are the detector coordinates of the track vertex, and $X=1.4828 \mathrm{~m}$ and $Y=0.2384 \mathrm{~m}$ are the coordinates of the neutrino beam center at the front face of the near detector. This fiducial volume was adopted to minimize reconstruction errors and improve the measurement of event energy [103]. These two goals are achieved by using the smaller fiducial volume, which improves the containment of secondary particles produced in neutrino interactions. The fiducial volume mass is 23.7 tons. For the oscillation analysis, the uncertainty of the near detector fiducial mass is included in the overall event normalization uncertainty of $4 \%$, for the predicted far detector event rate.

The neutral-current events are rejected using the muon identification technique described in Chapter 4 . The $\nu_{\mu}$ and $\bar{\nu}_{\mu}$ charged-current events are

\footnotetext{
${ }^{2}$ If the event contains more than one track then the longest track is used.
} 


\begin{tabular}{l|r|r} 
Type & Charge sign & Additional requirements \\
\hline$\nu_{\mu}$ charged-current & $\frac{q / p}{\sigma(q / p)}<-2$ & \\
\hline$\nu_{\mu}$ charged-current QES & $\frac{q / p}{\sigma(q / p)}<-2$ & $E_{\text {had }}<150 \mathrm{MeV}$ \\
\hline$\nu_{\mu}$ charged-current RES & $\frac{q / p}{\sigma(q / p)}<-2$ & $E_{\text {had }}>150 \mathrm{MeV}$ and $W^{2}<4 \mathrm{GeV}^{2}$ \\
\hline$\nu_{\mu}$ charged-current DIS & $\frac{q / p}{\sigma(q / p)}<-2$ & $E_{\text {had }}>150 \mathrm{MeV}$ and $W^{2}>4 \mathrm{GeV}^{2}$ \\
\hline $\bar{\nu}_{\mu}$ charged-current & $\frac{q / p}{\sigma(q / p)}>+2$ & $N_{U}>19$ and $N_{V}>19$ and $1<\phi<5$
\end{tabular}

Table 6.1: Selection criteria for the five categories of events used in our analysis of the near and far detector data: $\nu_{\mu}$ charged-current, $\bar{\nu}_{\mu}$ charged-current, and $\nu_{\mu}$ charged-current QES, RES, and DIS events. The charge sign variables $\frac{q / p}{\sigma(q / p)}$ and $\phi$ are defined in Chapter 5. The invariant mass squared $W^{2}$ is defined in Section 3.3. $N_{U}\left(N_{V}\right)$ is the number of track hits in the $\mathrm{U}(\mathrm{V})$ detector view.

selected using the muon charge sign method described in Chapter 5. The $\nu_{\mu}$ charged-current events can be further subdivided into the QES, RES, and DIS events using the technique developed in Section 3.3. The selection criteria for these five event categories are summarized in Table 6.1.

In Table 6.1, a primary event selection method is described. This selection method can be altered by changing the selection requirements listed in Table 6.1. The difference between the primary and altered methods is used to evaluate systematic errors for the event selection. The following variations produce nine additional sets of selected events: (a) the $P_{S}$ requirement is changed by \pm 0.1 ; (b) the $E_{\text {had }}$ is changed by $\pm 100 \mathrm{MeV}$; (c) the $W^{2}$ requirement is changed by $\pm 0.5 \mathrm{GeV}^{2}$; (d) the number of planes requirement is changed by \pm 5 ; (e) the requirement for stopping track selection is relaxed to select more tracks. A complete analysis of the near detector data is performed for each of the nine additional sets of events. This analysis includes the tuning of the MC 

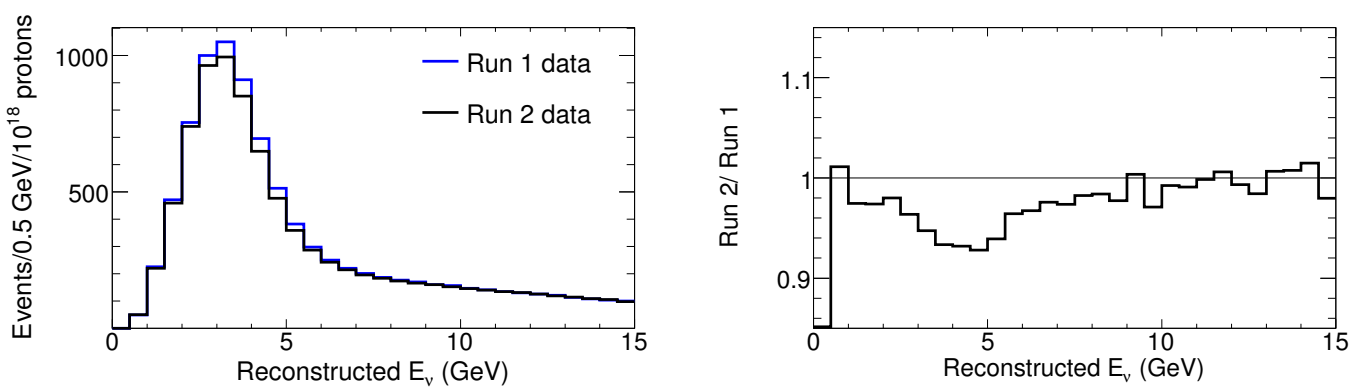

Figure 6.6: Energy spectra for the Run I and Run II data. The target position in Run II was approximately $1 \mathrm{~cm}$ closer to the first horn. This shift changes focusing characteristics for the secondary pions and produces fewer neutrinos. To account for this difference in target position, separate analysis procedures were performed for the Run I and Run II data.

simulation, as described in Chapter 7.

\subsection{Near detector data}

Our analysis uses all the near detector data recorded between May 2005 and July 2007. The integrated number of protons on target as a function of time is shown in Figure 2.2. This period was divided into two shorter periods, Run I and Run II, which were separated by a technical shutdown of the accelerator complex at Fermilab in the Summer of 2006. During this shutdown, the original target was replaced with a target of identical construction because the target's motion mechanism failed. The new target was placed approximately $1 \mathrm{~cm}$ closer to the first horn resulting in a $30 \mathrm{MeV}$ shift in the neutrino energy peak for the low energy (LE) beam configuration. The energy spectra for the Run I and Run II data are shown in Figure 6.6. 


\begin{tabular}{l|l|r|r} 
Beam configuration & Run period & Data POT $\left(\times 10^{18}\right)$ & MC POT $\left(\times 10^{18}\right)$ \\
\hline $\mathrm{L} 10 \mathrm{~cm} / 0 \mathrm{kA}$ & 1 & 2.77 & 38.37 \\
\hline $\mathrm{L} 10 \mathrm{~cm} / 0 \mathrm{kA}$ & 2 & 2.75 & 38.37 \\
\hline $\mathrm{L} 10 \mathrm{~cm} / 170 \mathrm{kA}$ & 1 & 1.44 & 3.54 \\
\hline $\mathrm{L} 10 \mathrm{~cm} / 185 \mathrm{kA}$ & 1 & 126.01 & 193.60 \\
\hline $\mathrm{L} 10 \mathrm{~cm} / 185 \mathrm{kA}$ & 2 & 169.13 & 193.60 \\
\hline $\mathrm{L} 10 \mathrm{~cm} / 200 \mathrm{kA}$ & 1 & 1.35 & 3.58 \\
\hline $\mathrm{L} 100 \mathrm{~cm} / 200 \mathrm{kA}$ & 1 & 1.02 & 3.51 \\
\hline $\mathrm{L} 150 \mathrm{~cm} / 200 \mathrm{kA}$ & 2 & 1.87 & 3.49 \\
\hline $\mathrm{L} 250 \mathrm{~cm} / 200 \mathrm{kA}$ & 2 & 15.46 & 50.38
\end{tabular}

Table 6.2: This table shows the beam configurations used for the analysis and the number of protons on target (POT) for the data and MC simulation.

Table 6.2 lists the beam configurations and the number of protons on target $(\mathrm{POT})$ in each beam configuration. The majority of the data were recorded in the low energy (LE) beam configuration. The MC events are scaled, independently for each beam configuration, by a ratio of the number of POT in the data divided by the number of POT in the MC simulation.

The remaining figures in this chapter show the selected $\nu_{\mu}$ chargedcurrent events for the data and MC simulation (default and tuned). Figures 6.7 and 6.8 show the reconstructed energy $E_{\nu}$, hadronic shower energy $E_{\text {had }}$, muon momentum $P_{\mu}$, and reconstructed muon angle $\theta_{\mu}$. These figures show events from the Run I and Run II data, recorded in the low energy beam configuration. In the reconstructed energy spectrum, there are two regions where the data and default MC simulation disagree. First, there are approximately $20 \%$ more data events with energy less than $4 \mathrm{GeV}$. Appendix A compares the data and MC energy spectra for several values of the Bjorken scaling variable, $X$, and 
the momentum transfered squared variable, $Q^{2}$. These comparisons indicate that the differences between the data and MC simulation below $4 \mathrm{GeV}$ depend on these variables. This fact suggests that in this energy region the neutrino cross-sections are not correctly modeled.

There are approximately $40 \%$ more data events with energy greater than $4 \mathrm{GeV}$. These disagreements depend neither on the Bjorken scaling variable, $X$, nor the momentum transfered squared variable, $Q^{2}$. This fact suggests that these differences are caused by an incorrect modeling of the NuMI neutrino flux, as demonstrated by the previous analysis $[58,101]$.

The kinematic variables, defined in Section 3.3, are shown in Figures 6.9 and 6.10. These distributions are not used by the tuning procedure to minimize differences between the data and the MC simulation. The agreement between the data and the MC simulation significantly improved after the tuning, especially for the invariant mass squared variable that is used to select the RES events. For the reconstructed $Q^{2}$ variable, the data contain fewer events for $Q^{2}<200 \mathrm{MeV}$, after the tuning. For other variables, there also exist regions with substantial differences between the data and MC events. A source of these differences is currently unknown.

The reconstructed energy spectra for the selected QES, RES, and DIS events are shown in Figures 6.11 and 6.13. These spectra are used by the tuning procedure to minimize differences between the data and the MC simulation. Figures 6.15 and 6.16 show the energy spectra for the additional beam configurations, used by the tuning procedure. These figures demonstrate that 
the tuning procedure produces an accurate description of the near detector data, with the exception of the low energy events below $1 \mathrm{GeV}$. These low energy events are susceptible to nuclear effects that tend to suppress neutrino interaction rates.

A bin error for the tuned MC histograms is a sum of statistical and systematic errors. The statistical error is the square root of a number of entries in the bin. The systematic error is determined by varying the event selection requirements, as described in Section 6.2. For each set of the selection requirements, the histograms are filled with the data and $\mathrm{MC}$ events and a tuning procedure is performed using these histograms; then, a new set of the histograms is created and filled with the tuned MC events. The systematic error in each bin is the maximum deviation of any of the additional histograms from the histogram obtained with the primary selection method. 

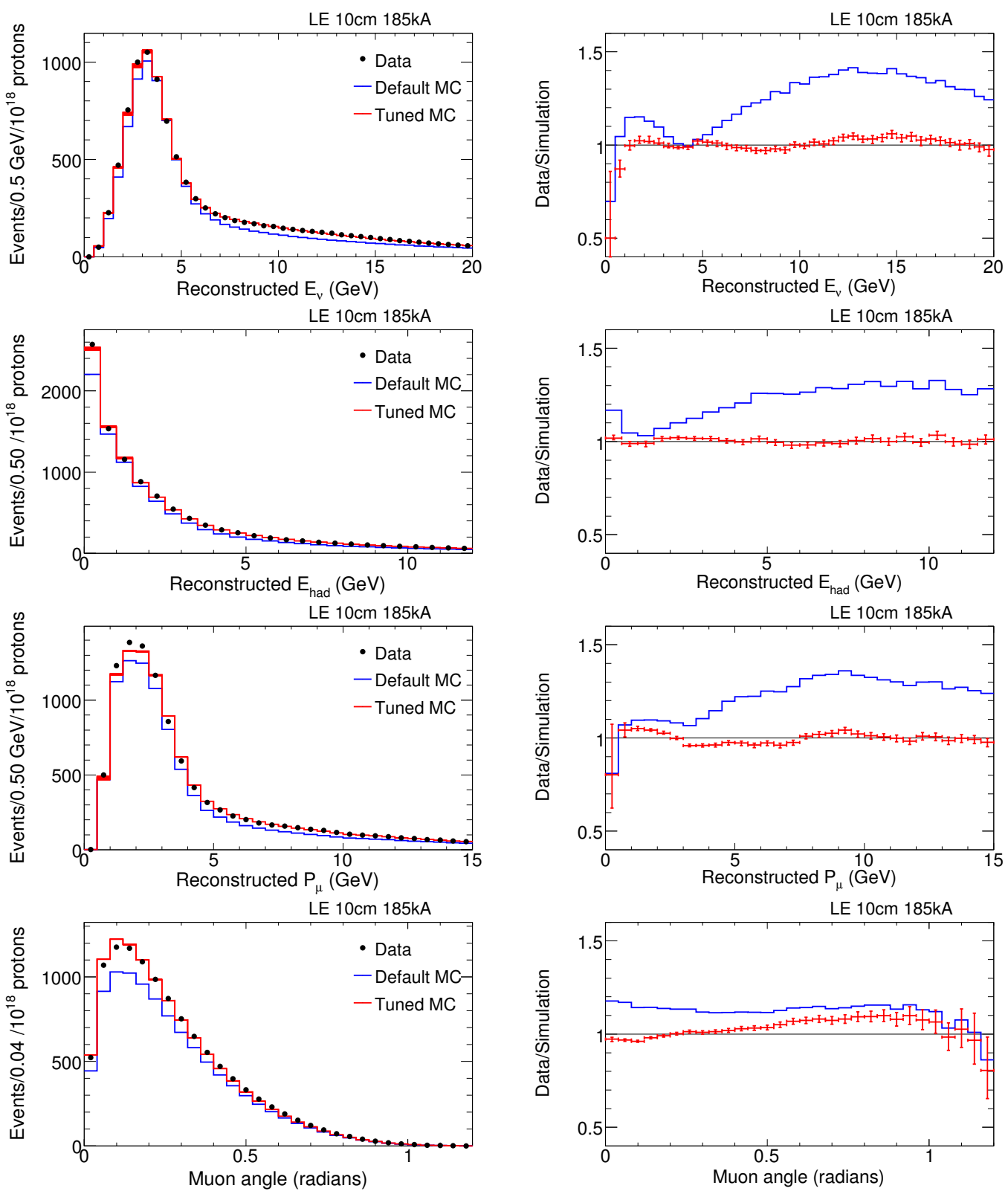

Figure 6.7: Reconstructed event energy $E_{\nu}$, hadronic shower energy $E_{\text {had }}$, muon momentum $P_{\mu}$ and reconstructed muon angle for the selected $\nu_{\mu}$ chargedcurrent events. These figures show the Run I near detector data. The right plots show the data divided by the default and tuned MC simulation. 

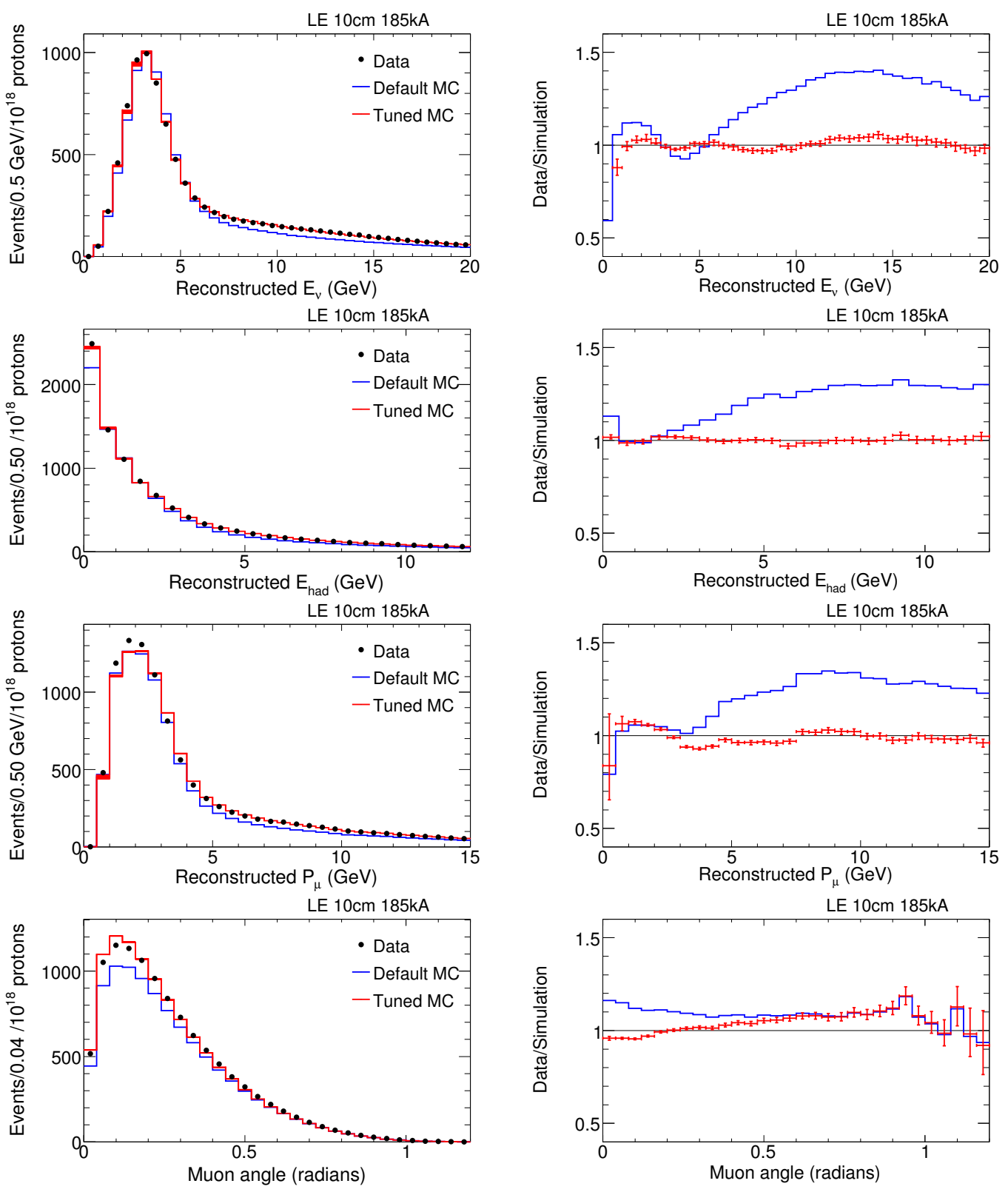

Figure 6.8: Reconstructed event energy $E_{\nu}$, hadronic shower energy $E_{\text {had }}$, muon momentum $P_{\mu}$ and reconstructed muon angle for the selected $\nu_{\mu}$ chargedcurrent events. These figures show the Run II near detector data. The right plots show the data divided by the default and tuned MC simulation. 

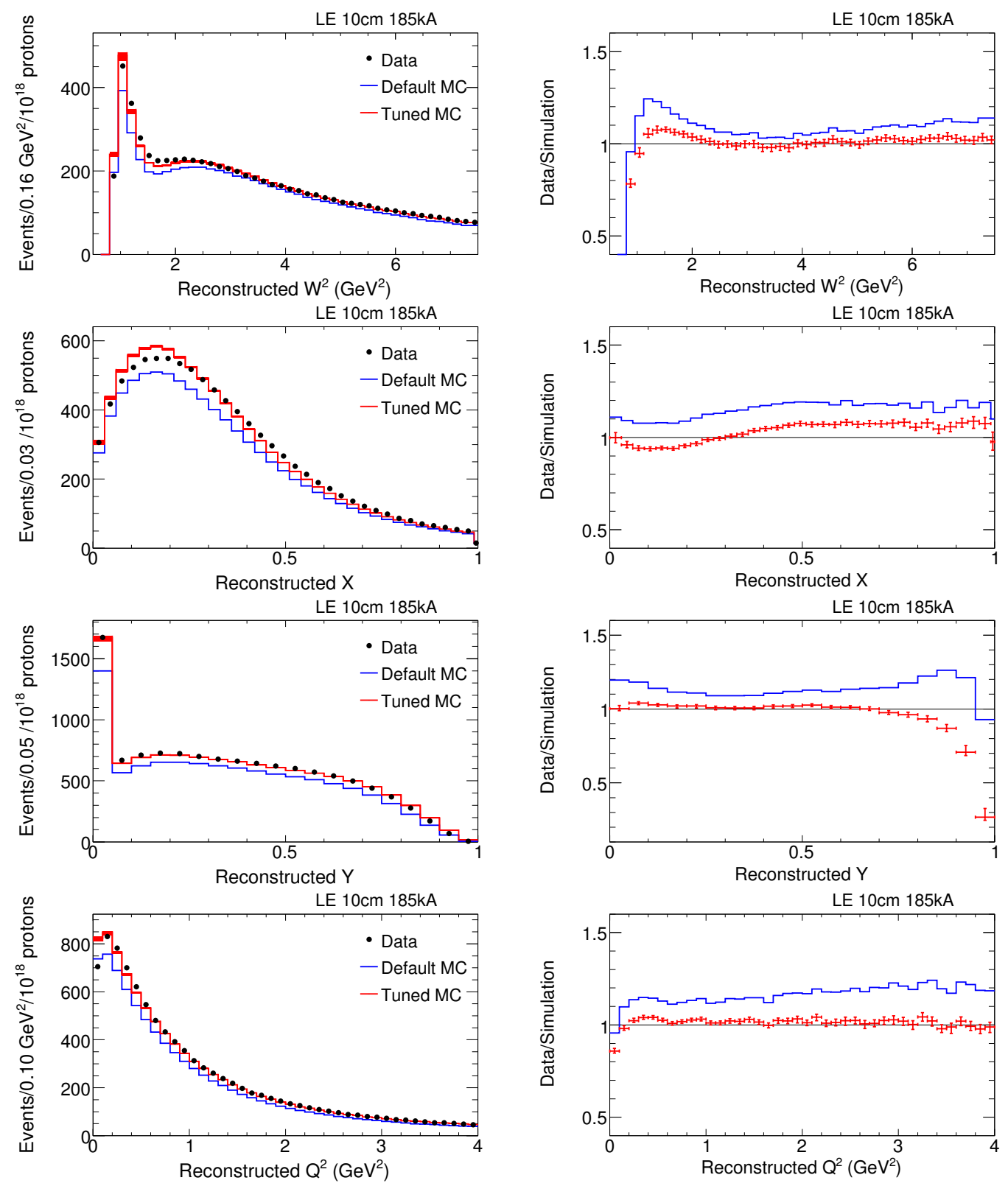

Figure 6.9: Reconstructed $W^{2}, X, Y$, and $Q^{2}$ distributions for the selected $\nu_{\mu}$ charged-current events. These figures show the Run I near detector data. The right plots show the data divided by the default and tuned MC simulation. 

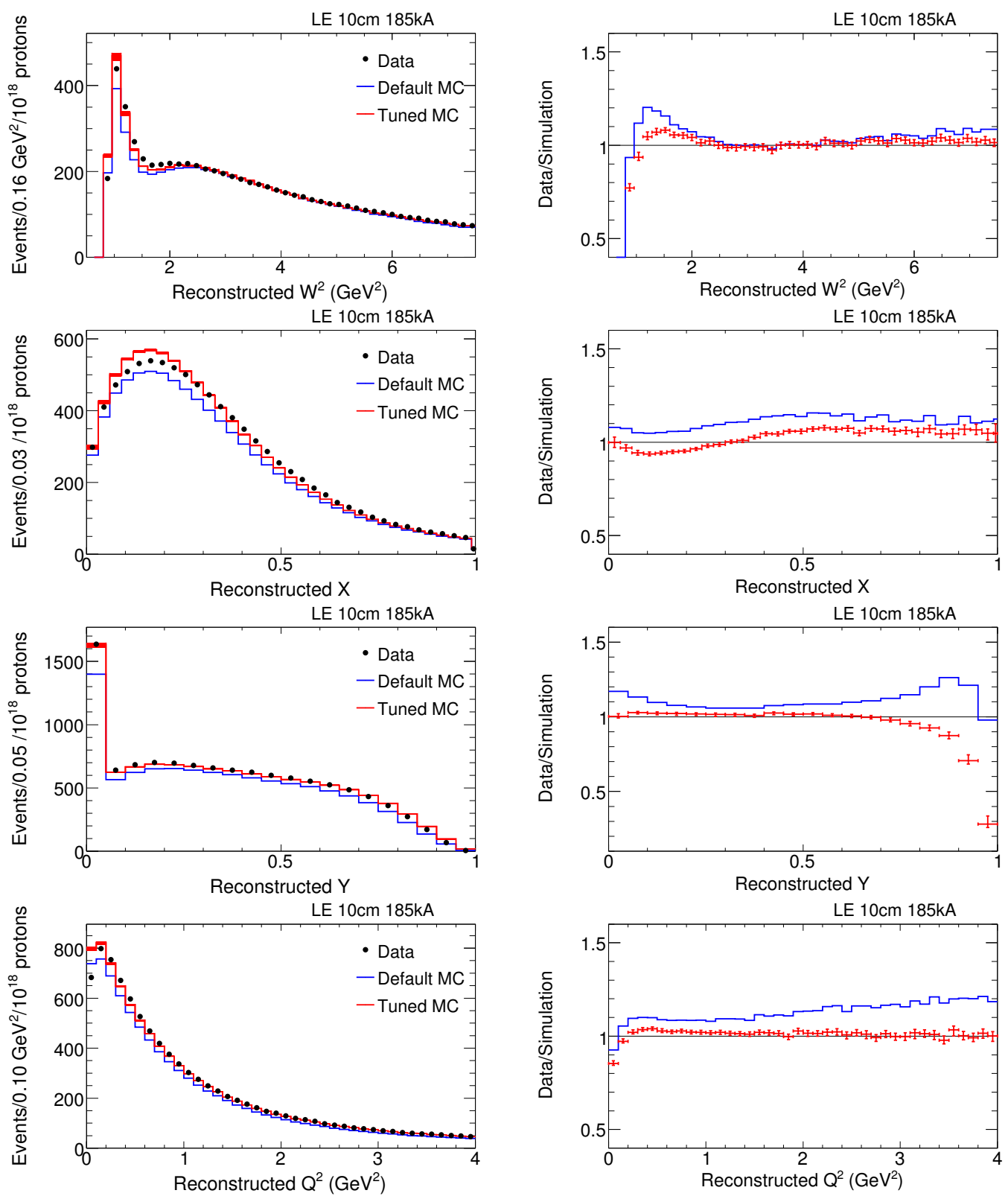

Figure 6.10: Reconstructed $W^{2}, X, Y$, and $Q^{2}$ distributions for the selected $\nu_{\mu}$ charged-current events. These figures show the Run II near detector data. The right plots show the data divided by the default and tuned $\mathrm{MC}$ simulation. 

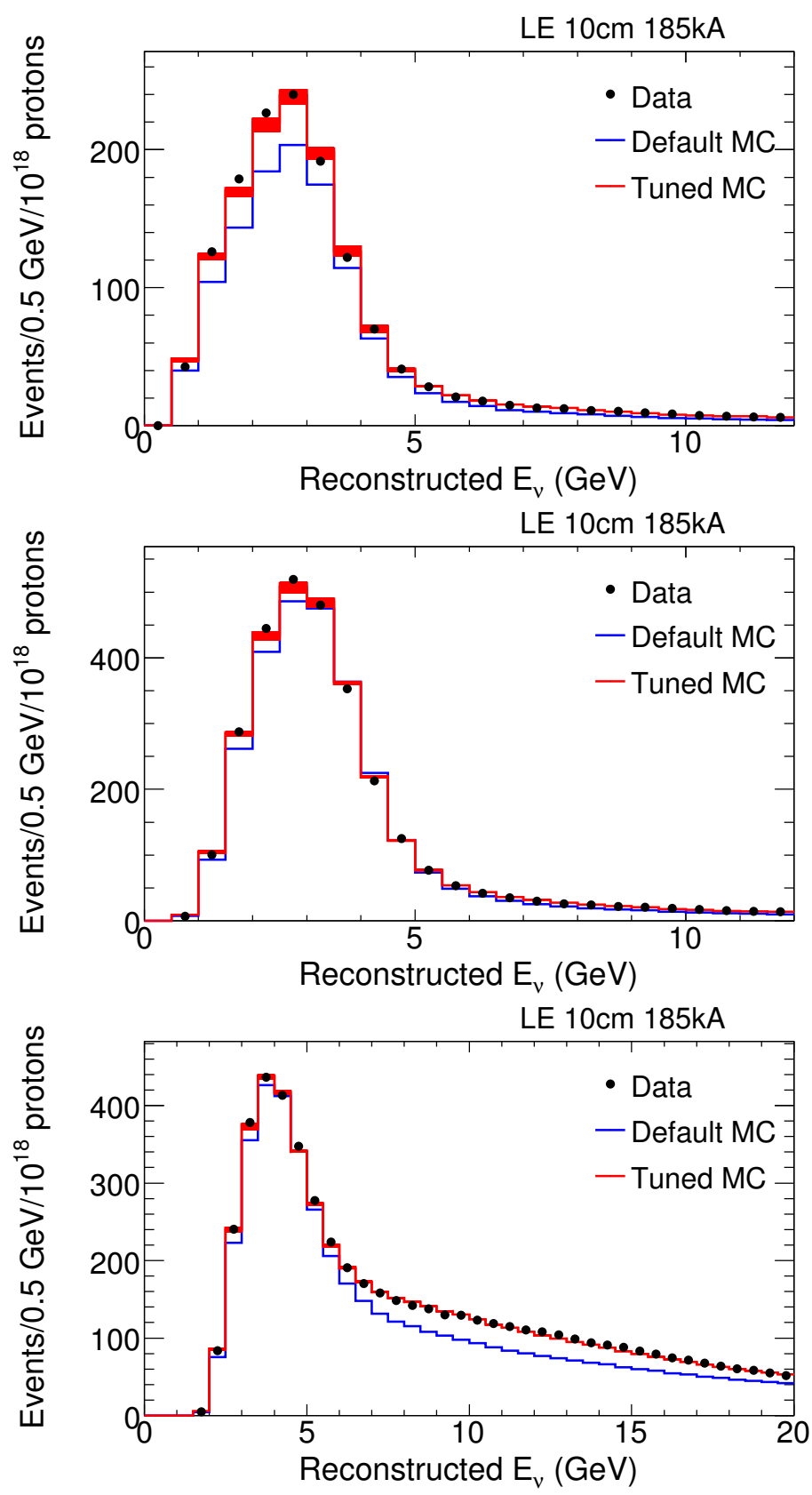

Figure 6.11: Reconstructed energy $E_{\nu}$ for the selected QES, RES, and DIS events. These figures show the Run I near detector data. The top figure shows the selected QES events. The middle figure shows the selected RES events. The bottom figure shows the selected DIS events. 

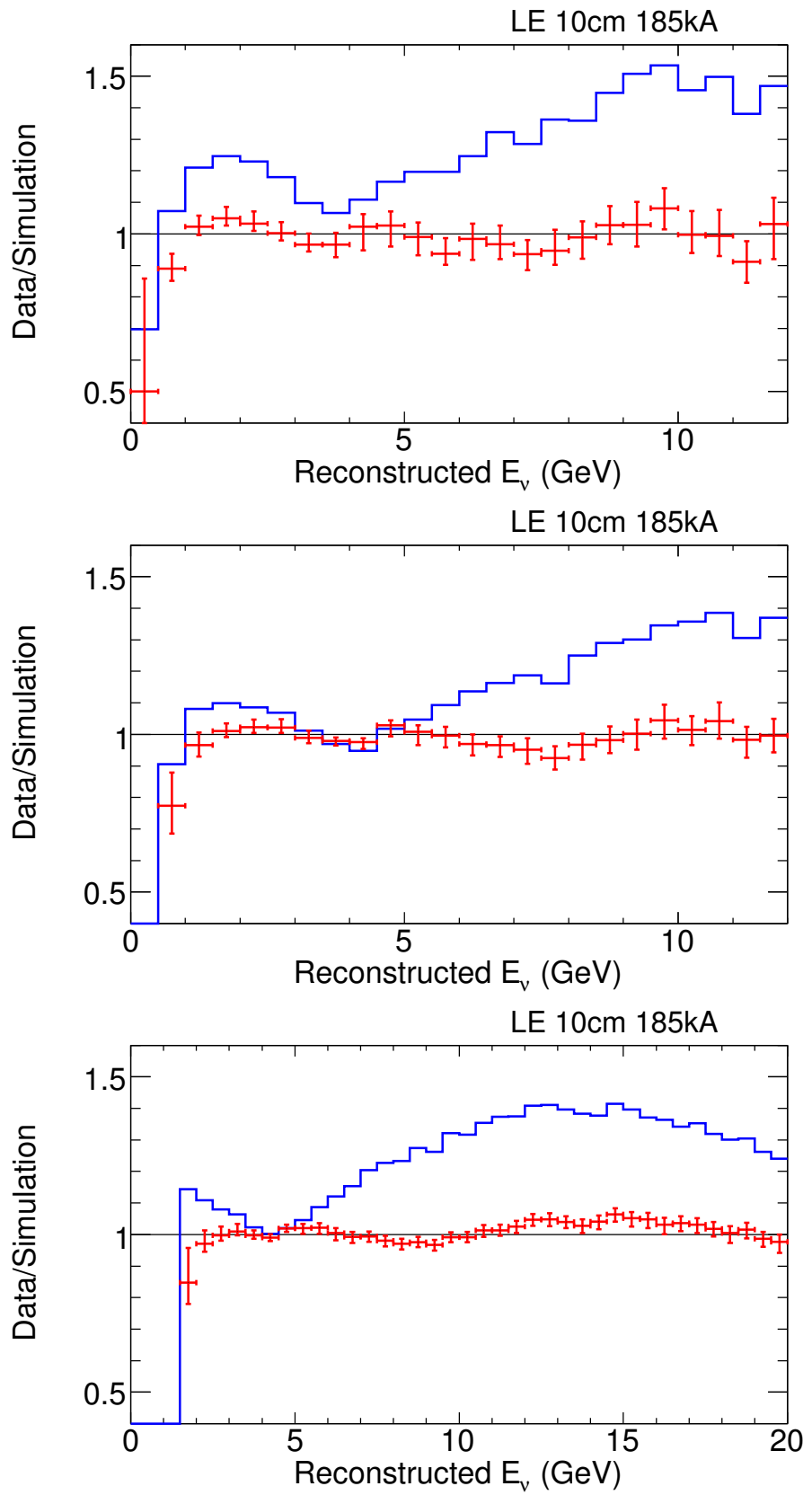

Figure 6.12: These figures show the data divided by the default and tuned MC simulation, for the reconstructed energy $E_{\nu}$. These figures show the Run I near detector data. The top figure shows the selected QES events. The middle figure shows the selected RES events. The bottom figure shows the selected DIS events. 

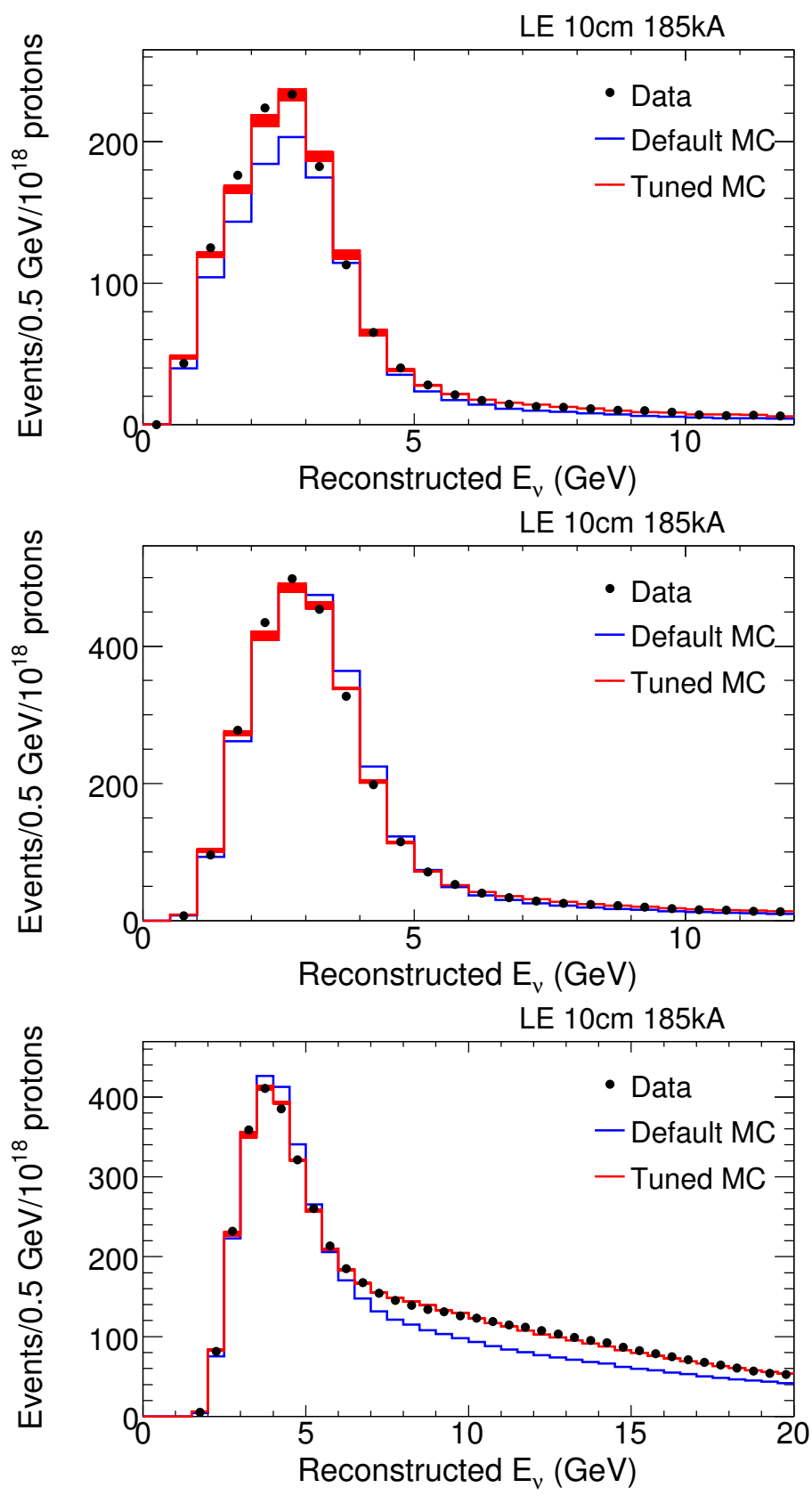

Figure 6.13: Reconstructed energy $E_{\nu}$ for the selected QES, RES, and DIS events. These figures show the Run II near detector data. The top figure shows the selected QES events. The middle figure shows the selected RES events. The bottom figure shows the selected DIS events. 

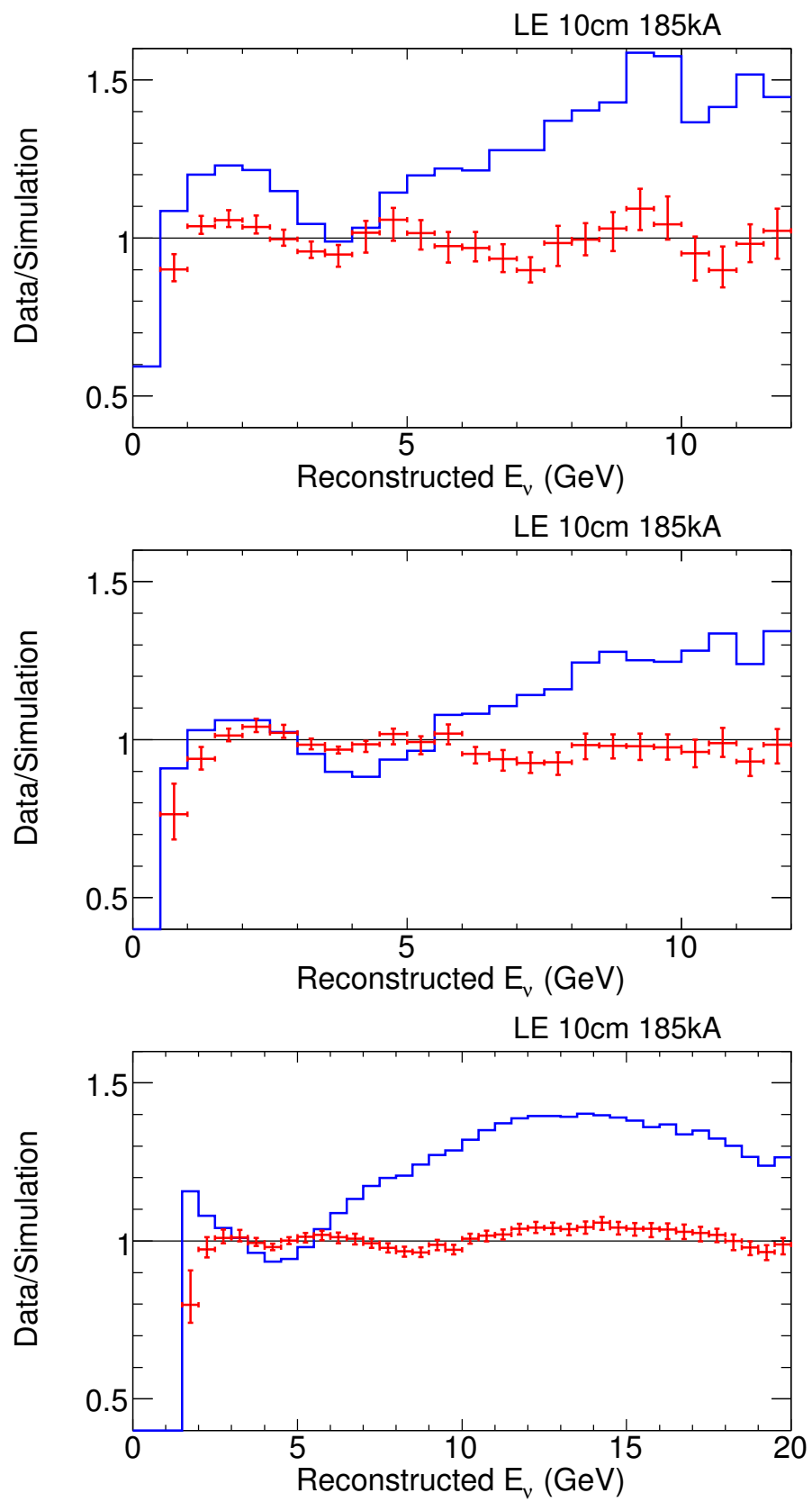

Figure 6.14: These figures show the data divided by the default and tuned MC simulation, for the reconstructed energy $E_{\nu}$. These figures show the Run II near detector data. The top figure shows the selected QES events. The middle figure shows the selected RES events. The bottom figure shows the selected DIS events. 

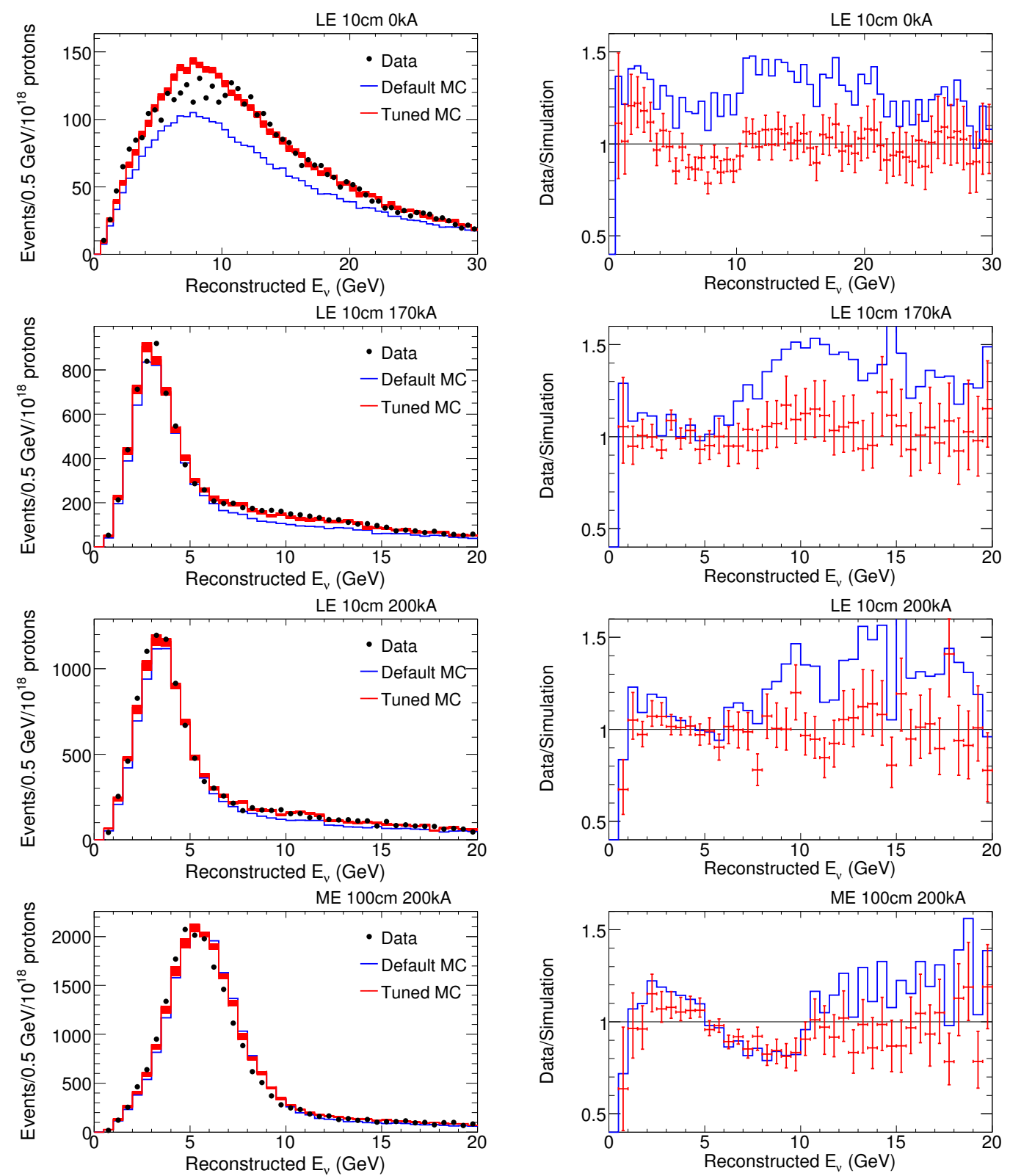

Figure 6.15: Reconstructed energy $E_{\nu}$ for the $\nu_{\mu}$ charged-current events. These figures show the Run I near detector data. Four different beam configurations are shown, as labeled on the figures. The right plots show the data divided by the default and tuned MC simulation. 

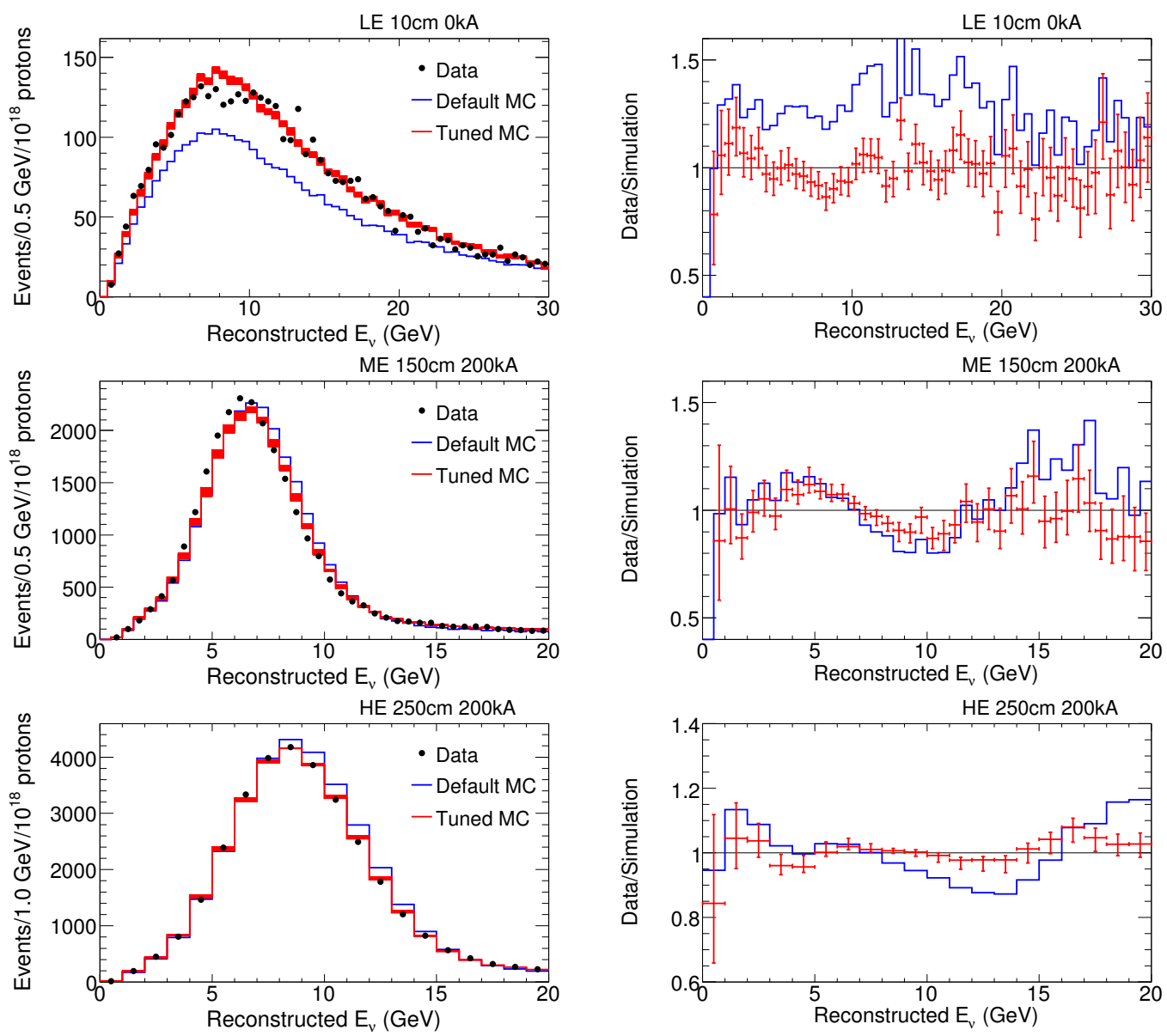

Figure 6.16: Reconstructed energy $E_{\nu}$ for the $\nu_{\mu}$ charged-current events. These figures show the Run II near detector data. Three different beam configurations are shown, as labeled on the figures. The right plots show the data divided by the default and tuned MC simulation. 


\subsection{Summary}

This chapter examined the near detector $\nu_{\mu}$ charged-current events. First, a study of the near detector events resulted in an improved understanding of the magnetic field in the near detector. Then, distributions of the near detector observables from the neutrino interactions were examined. These observables will be used in chapter Chapter 7 to compute corrections to the MC simulation. In Chapters 8 and 9, these corrections will be used in the oscillation analysis of the far detector events. 


\section{Chapter 7}

\section{Tuning of flux and cross-section models}

MINOS is a two-detector neutrino oscillation experiment that uses two spatially separated detectors to observe the NuMI neutrino beam. In essence, MINOS measures the disappearance of muon neutrinos via a counting experiment with two similar detectors. This measurement requires a prediction of the event rate at the far detector in the absence of neutrino oscillations. The near detector data are utilized to compute this prediction. This computation is called an extrapolation from the near detector to the far detector. This usage of the MINOS near detector data results in a partial cancellation of systematic errors from the incorrect modeling of the detector, neutrino flux, and cross-sections, as well as the mismeasurement of event observables [58].

The MINOS experiment utilizes two general approaches to extrapolate the near detector data to the far detector [58]: direct methods and tuning methods. The tuning (or fitting) method analyzes the near detector data and adjusts accordingly parameters of MC simulation models. The corrected (tuned) MC simulation is then used to compute the predicted far detector event rate as a function of the reconstructed neutrino energy. The present analysis uses a tuning method. 
This chapter describes the tuning procedure that minimizes the differences between the near detector data and the MC simulation by adjusting the neutrino flux and cross-section models. The present analysis is an improvement over the previously developed tuning methods [58,80,101,104]. Our analysis treats separately quasi-elastic scattering (QES), resonance production (RES), and deep-inelastic scattering (DIS) events. These events are separated into three categories, using the technique described in Chapter 3. The resulting tuning procedure has some sensitivity to cross-sections for these processes. The measurement of an event rate for two of these processes, QES and RES events, improves our understanding of the $\nu_{\mu}$ charged-current interactions in the MINOS detectors. In addition, our far detector oscillation analysis utilizes these event categories for a measurement of oscillation parameters, as described in Chapter 9.

In this chapter, the tuning parameters for the muon neutrino flux and cross-section models are described in Sections 7.1 and 7.2, and the following sections present tuning results.

\subsection{Parameterization of the flux model}

The NuMI muon neutrino beam is generated by the decay of pions and kaons within the decay pipe. The energy spectrum of this neutrino beam is determined by the momentum distribution of pions and kaons produced by the interaction of $120 \mathrm{GeV}$ protons with the NuMI target. In addition, the relative position of the target and magnetic horns determines the momentum 
of hadrons focused in the forward direction, as discussed in Section 2.2. The predicted contributions of pions and kaons to the muon neutrino flux at the near detector are shown in Figure 2.5.

Figure 7.1 illustrates the focusing effect of the magnetic horns on the $\nu_{\mu}$ flux. This figure shows the transverse, $p_{T}$, and forward, $p_{z}$, momentum components for the MC $\pi^{ \pm}$that decay into the neutrinos that reach the near detector. These momentum components are computed at the point where these hadrons exit the NuMI target. This figure includes four beam configurations. In the LE $10 \mathrm{~cm} / 0 \mathrm{kA}$ beam configuration, ${ }^{1}$ the focusing horns are turned off. As a result, only forward going pions with $p_{T} \approx 50 \mathrm{MeV}$ produce neutrinos that reach the near detector. In other beam configurations, the magnetic horns are activated, selecting hadrons with higher $p_{T}$ values. The magnetic horns focus $\pi^{+}$toward the decay pipe, and the $\pi^{-}$are focused toward the walls of the decay pipe. If the target is pulled further away from the horns, $\pi^{+}$with the higher $p_{z}$ momentum are focused toward the decay pipe; this results in a neutrino flux with a higher average neutrino energy. The data from different beam configurations sample different regions of the $p_{T}$ and $p_{z}$ plane. Similar focusing effects are present for kaons, but the overall focusing strength is reduced because kaons are heavier than pions.

Differences between the design specifications for the NuMI beamline and the actual constructed elements produce disagreements between the data

\footnotetext{
${ }^{1}$ This configuration is labeled as LE010z_0kA in the figures. The naming convention is explained in Section 2.2.
} 

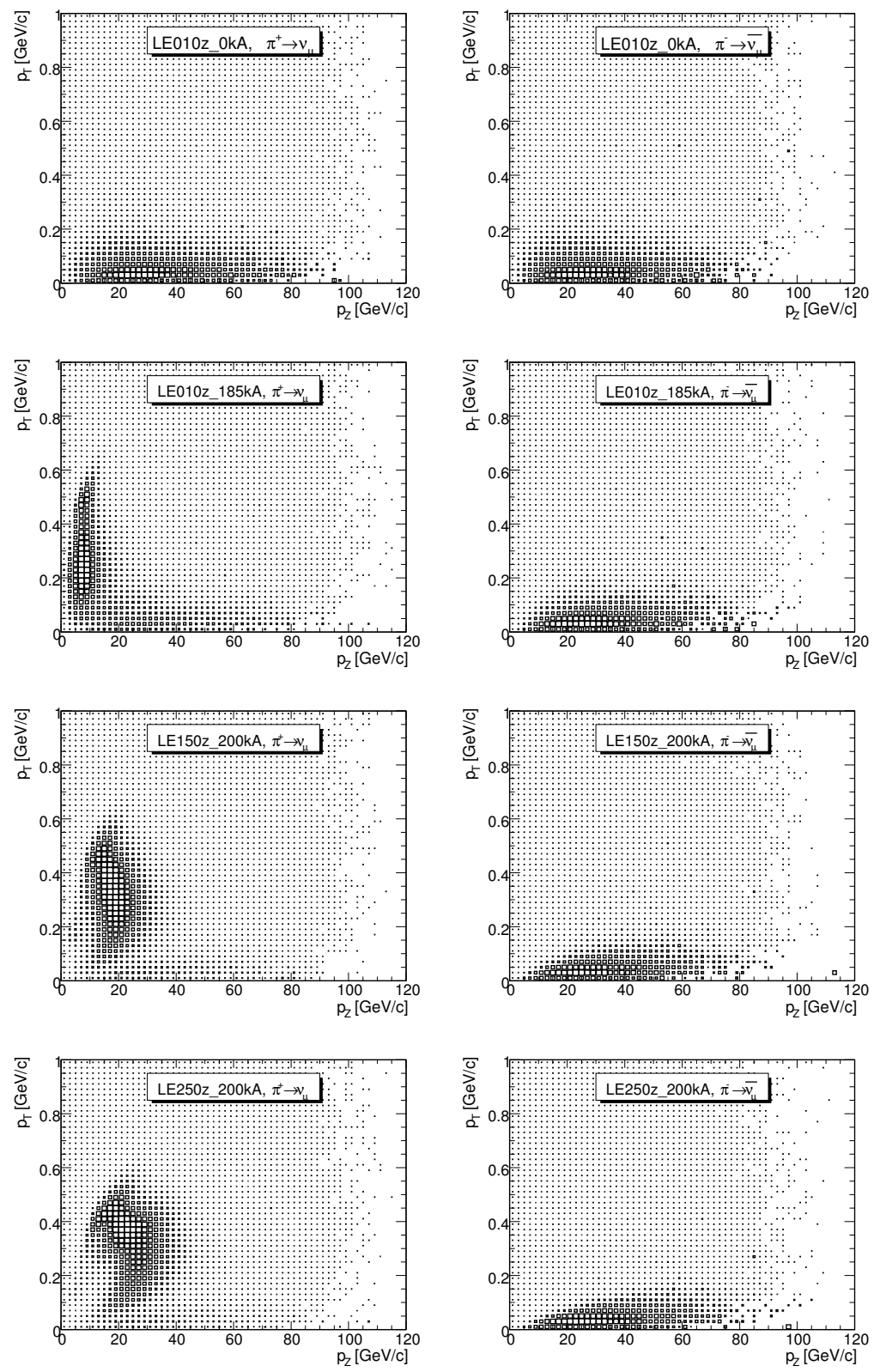

Figure 7.1: Scatter plots of $p_{T}$ versus $p_{z}$ momentum components for the parent pions that produce neutrinos at the near detector, as calculated by FLUKA05. The left plots show the contributions to the near detector $\nu_{\mu}$ spectra from $\pi^{+}$ decays. The right plots show the contributions to the near detector $\bar{\nu}_{\mu}$ spectra from $\pi^{-}$decays. These figures were taken from [63]. 
and expected flux from the MC simulation. The study of these beamline effects concluded that the error of the predicted far detector flux is less than 3\% [101] if the near detector data are used to generate the prediction. These effects contribute the negligably small systematic error for the measurement of the oscillation parameters [58]. This error was not included in the present analysis.

There are substantial differences between the data and (default) MC simulation for the $\nu_{\mu}$ charged-current energy spectra from several beam configurations. These energy spectra are shown in Chapter 6. The magnitude of these disagreements varies with energy and depends on the beam configuration. The largest differences between the data and MC simulation result from the incorrect MC computation of the neutrino flux [58]. The uncertainty in the yield of hadrons from the NuMI target directly translates into the uncertainty of the muon neutrino flux. Hadron production data for the NuMI target are not currently available. A procedure was developed to employ the near detector neutrino data ${ }^{2}$ to constrain the hadron production [101]. This procedure is used by the present analysis to constrain the muon neutrino flux. The main feature of our analysis is a separate treatment of the QES, RES, and DIS events, as discussed next in Section 7.2.

The $\nu_{\mu}$ and $\bar{\nu}_{\mu}$ flux is constrained using the $\nu_{\mu}$ charged-current and $\bar{\nu}_{\mu}$ charged-current events from several beam configurations. The yield of pions and kaons from the NuMI target is parametrized by the following function [105,

\footnotetext{
${ }^{2}$ The NuMI beamline includes ionization chambers that monitor muon flux. These monitors can also measure muon neutrino flux through a measurement of the muon flux.
} 
106]:

$$
\frac{d^{2} N}{d p_{z} d p_{T}}=\left(A\left(x_{F}\right)+p_{T} B\left(x_{F}\right)\right) e^{-C\left(x_{F}\right) p_{T}^{3 / 2}}
$$

where $p_{z}$ is the forward momentum component, $p_{t}$ is the transverse momentum component, and $x_{F} \approx p_{z} / 120 \mathrm{GeV}$ is the fraction of maximum available momentum in the lab frame carried by the hadron. The $A$ parameter controls the hadron yield for the low $p_{T}$ values; the $B$ parameter determines the rising slope of hadron yield with an increasing $p_{T}$ value; and, the $C$ parameter controls the fall off behavior at the large $p_{T}$ values. The parameters $A, B$, and $C$ are functions of $x_{F}$, given by:

$$
\begin{aligned}
& A\left(x_{F}\right)=a_{1}\left(1-x_{F}\right)^{a_{2}}\left(1+a_{3} x_{F}\right) x_{F}^{-a_{4}}, \\
& B\left(x_{F}\right)=b_{1}\left(1-x_{F}\right)^{b_{2}}\left(1+b_{3} x_{F}\right) x_{F}^{-b_{4}}, \\
& C\left(x_{F}\right)= \begin{cases}c_{1} x_{F}^{-c_{2}}+c_{3} & \text { if } x_{F}<0.22, \\
c_{1} e^{c_{2}\left(x_{F}-c_{3}\right)}+c_{4} x_{F}+c_{5} & \text { if } x_{F}>0.22,\end{cases}
\end{aligned}
$$

where the constants $a_{i}, b_{i}$ and $c_{i}$ were obtained by fitting the predicted hadron production yield for $\pi^{+}$and $K^{+}$[105], as computed by FLUKA05. This parametrization provides a satisfactory description of the FLUKA05 hadron production model. The flux tuning procedure does not require a precise agreement between a fitted distribution and the FLUKA05 output because the hadron production is adjusted relative to the FLUKA05 model, as described next.

The tuning procedure adjusts the yield of the pions and kaons off the NuMI target by assigning an importance weight to individual hadrons. The weights are adjusted relative to the output of the FLUKA05 simulation. This 
procedure uses the hadron type $\left(\pi^{ \pm}\right.$and $\left.K^{ \pm}\right)$and the $p_{T}$ and $x_{F}$ values. The yield of $\pi^{+}$is adjusted with the 6 parameters $\left(p_{0}\right.$ through $\left.p_{5}\right)$ :

$$
\begin{aligned}
& A_{\pi^{+}}^{\prime}\left(x_{F}\right)=\left(p_{0}+p_{1} x_{F}\right) A_{\pi^{+}}(x), \\
& B_{\pi^{+}}^{\prime}\left(x_{F}\right)=\left(p_{2}+p_{3} x_{F}\right) B_{\pi^{+}}(x), \\
& C_{\pi^{+}}^{\prime}\left(x_{F}\right)=\left(p_{4}+p_{5} x_{F}\right) C_{\pi^{+}}(x) .
\end{aligned}
$$

The yield of $K^{+}$is adjusted with the 6 parameters $\left(p_{6}\right.$ through $\left.p_{11}\right)$ :

$$
\begin{aligned}
& A_{K^{+}}^{\prime}\left(x_{F}\right)=\left(p_{6}+p_{7} x_{F}\right) A_{K^{+}}(x), \\
& B_{K^{+}}^{\prime}\left(x_{F}\right)=\left(p_{8}+p_{9} x_{F}\right) B_{K^{+}}(x), \\
& C_{K^{+}}^{\prime}\left(x_{F}\right)=\left(p_{10}+p_{11} x_{F}\right) C_{K^{+}}(x) .
\end{aligned}
$$

The importance weight for $\pi^{+}$and $K^{+}$is calculated as follows:

$$
\begin{aligned}
W_{\pi^{+}}\left(p_{T}, x_{F}\right) & =\frac{\left(A_{\pi^{+}}^{\prime}\left(x_{F}\right)+B_{\pi^{+}}^{\prime}\left(x_{F}\right) p_{T}\right) e^{-C_{\pi^{+}}^{\prime}\left(x_{F}\right) p_{T}^{3 / 2}}}{\left(A_{\pi^{+}}\left(x_{F}\right)+B_{\pi^{+}}\left(x_{F}\right) p_{T}\right) e^{-C_{\pi^{+}}\left(x_{F}\right) p_{T}^{3 / 2}}} \\
W_{K^{+}}\left(p_{T}, x_{F}\right) & =\frac{\left(A_{K^{+}}^{\prime}\left(x_{F}\right)+B_{K^{+}}^{\prime}\left(x_{F}\right) p_{T}\right) e^{-C_{K^{+}}^{\prime}\left(x_{F}\right) p_{T}^{3 / 2}}}{\left(A_{K^{+}}\left(x_{F}\right)+B_{K^{+}}\left(x_{F}\right) p_{T}\right) e^{-C_{K^{+}}\left(x_{F}\right) p_{T}^{3 / 2}}} .
\end{aligned}
$$

The near detector data have little sensitivity to the negatively charged hadrons. The magnetic horns focus these particles toward the decay pipe walls, and only the forward going hadrons produce anti-neutrinos that reach the near detector, as illustrated in Figure 7.1 for $\pi^{-}$. The $\pi^{+} / \pi^{-}$and $K^{+} / K^{-}$ ratios are expected to be better constrained by the experimental data used in the FLUKA05 simulation. As a result, for $\pi^{-}$and $K^{-}$the importance weight is adjusted with the 4 parameters $\left(p_{12}\right.$ through $\left.p_{15}\right)$ :

$$
\begin{gathered}
W_{\pi^{-}}\left(p_{T}, x_{F}\right)=\left(p_{12}+p_{13} x_{F}\right) W_{\pi^{+}}\left(p_{T}, x_{F}\right), \\
W_{K^{-}}\left(p_{T}, x_{F}\right)=\left(p_{14}+p_{15} x_{F}\right) W_{K^{+}}\left(p_{T}, x_{F}\right),
\end{gathered}
$$


where $W_{\pi^{+}}\left(p_{T}, x_{F}\right)$ and $W_{K^{+}}\left(p_{T}, x_{F}\right)$ are defined in Equation 7.7.

The initial (default) values of these 16 parameters are:

$$
p_{i}= \begin{cases}1, & \text { if } i=0,2,4,6,8,10,12,14, \\ 0, & \text { if } i=1,3,5,7,9,11,13,15 .\end{cases}
$$

For these initial values, Equations 7.7 and 7.8 return unity for any value of

$p_{T}$ and $p_{z}$. In this case, the default FLUKA05 flux is obtained. By changing the 16 parameters, the $p_{T}$ and $p_{z}$ plane is warped, and the importance weights of hadrons at the different momenta and angles is changed. This procedure effectively changes the yield of hadrons from the NuMI target. As a result, the muon neutrino flux at the detector are also changed. This tuning procedure minimizes the differences between the near detector data and MC simulation using these 16 flux parameters.

\subsection{Parameterization of the cross-section model}

The neutrino interaction rate in the detector is a function of the product of the neutrino flux and cross-section. As result, the MINOS near detector data alone are not sufficient to constrain simultaneously the absolute neutrino flux and the absolute cross-section normalization. The uncertainty of the normalization of the deep-inelastic scattering (DIS) cross-section is 3\%, for the DIS events with $W>1.7 \mathrm{GeV}$ and $E_{\nu}>30 \mathrm{GeV}$ [58]. For the low energy beam configuration, the predicted fraction of DIS events in the near detector is 
$56.8 \%{ }^{3}$ The DIS events are dominant for $E_{\nu}>6 \mathrm{GeV}$ (see Section 3.3). The tuning procedure leaves the normalization of the DIS events unchanged. As a result, the DIS events constrain the absolute flux normalization. However, the tuning procedure provides no information about the real normalization of the DIS cross-section in data below $30 \mathrm{GeV}$, where the DIS cross-section are not as accurately measured.

The normalization uncertainty of the quasi-elastic scattering (QES) cross-section is $10 \%$ [58]. The normalization uncertainty of the single-pion resonance production (RES) cross-section is 10\% [58]. For the low energy beam configuration, the predicted fraction of QES events is $17.6 \%$, and the predicted fraction of RES events is $25.6 \%$. These events are dominant for $E_{\nu}<6 \mathrm{GeV}$. The absolute normalization of the QES and RES events, from $\nu_{\mu}$ charged-current interactions, is adjusted using two parameters. These two parameters freely scale up and down the number of true QES and RES ${ }^{4}$ events in the MC simulation.

In the $E_{\nu}<6 \mathrm{GeV}$ energy region, nuclear effects for final-state hadrons produced in neutrino interaction with iron have a significant effect on the visible energy of the hadronic final state. In particular, there is a substantial uncertainty in the rate of pion absorption. The pion absorption can scramble the signature of the RES events in the MINOS detectors, as discussed in

\footnotetext{
${ }^{3}$ This estimate used the default MC simulation. The tuning procedure changes the high energy neutrino flux, which also changes the number of the DIS events in the near detector.

${ }^{4}$ This scale factor adjusts simultaneously the number of single-pion and higher mass resonances events.
} 
Section 3.3. The uncertainty of the visible hadronic energy from the final-state interactions is estimated by: (a) turning these effects off in the simulation; (b) modifying the simulation so that all of the absorbed pion energy is lost. The resulting changes in the hadronic shower energy response were approximately $10 \%$ [58]. Based on these studies, the normalization uncertainty of the QES and RES events is increased to $20 \%$ for the purpose of this tuning procedure.

The QES, RES, and DIS events, from the $\nu_{\mu}$ charged-current interactions, are separated into three categories using the technique developed in Section 3.3. These QES, RES, and DIS events are used to create three histograms, binned in the reconstructed neutrino energy. These histograms for the Run I and Run II data are shown in Figures 6.11 and 6.13, respectively. The tuning procedure minimizes the differences between the data and MC simulation, using these histograms. The data from several other beam configurations are also included in the fit, as summarized in Section 7.5.

The $\bar{\nu}_{\mu}$ charged-current events are included to constrain the production of $\pi^{-}$and $K^{-}$. These events are not used in the oscillation analysis. The $\bar{\nu}_{\mu}$ charged-current cross-section is unchanged by the fit. The $\bar{\nu}_{\mu}$ charged-current events are dominated by DIS events.

Four parameters are used to adjust the reconstructed neutrino energy scale: (a) three parameters scale the reconstructed energy, $E_{\nu}$, of the true QES, RES, and DIS events from $\nu_{\mu}$ charged-current interactions; (b) one parameter scales the reconstructed energy, $E_{\nu}$, of the true DIS events from $\bar{\nu}_{\mu}$ charged-current interactions. These four parameters are included to estimate 
the uncertainty of the reconstructed energy scale. The three separate scale factors for the QES, RES, and DIS events measure the energy scale uncertainty for different regions of neutrino energy. These events also produce hadronic showers with different energy scales, as discussed in Section 3.3. The separate energy scale factor for the $\bar{\nu}_{\mu}$ charged-current events is included because these events have a higher average energy than the $\nu_{\mu}$ charged-current events. In the MINOS detectors, the uncertainty of the reconstructed energy scale for the $\nu_{\mu}$ charged-current and $\bar{\nu}_{\mu}$ charged-current events is around $5 \%$ [58].

The 6 tuning parameters are summarized in Table 7.1, where the last column lists the uncertainty of these parameters. The tuning procedure changes the absolute normalization of the RES and QES events. The RES and QES cross-sections are rapidly changing functions of neutrino energy, as shown in Figure 3.8. The NuMI neutrino flux is also a rapidly changing function of neutrino energy, as shown in Figure 2.5. As a result, the energy dependence of the RES and QES cross-sections is difficult to measure with the MINOS near detector data. In addition, our results indicate that simple scale factors for the normalization of the QES and RES events are sufficient to describe the near detector data. Thus, it was decided to use the simple scale factors for the measurement of the QES and RES event rates in the MINOS near detector.

\subsection{Fit description and $\chi^{2}$ function}

This section briefly describes the fit procedure (fit) used to minimize the differences between the data and MC simulation. This fit uses the parameters 


\begin{tabular}{l|l|r} 
Parameter & Definition & Error \\
\hline \hline$p_{16}$ & $E_{\nu}$ scale for $\nu_{\mu}$ DIS events & $5 \%$ \\
\hline$p_{17}$ & $E_{\nu}$ scale for $\bar{\nu}_{\mu}$ DIS events & $5 \%$ \\
\hline$p_{18}$ & Normalization of $\nu_{\mu}$ QES cross-section & $20 \%$ \\
\hline$p_{19}$ & $E_{\nu}$ scale for $\nu_{\mu}$ QES events & $5 \%$ \\
\hline$p_{20}$ & Normalization of $\nu_{\mu}$ RES cross-section & $20 \%$ \\
\hline$p_{21}$ & $E_{\nu}$ scale for $\nu_{\mu}$ RES events & $5 \%$
\end{tabular}

Table 7.1: Summary of the 6 parameters used to adjust the cross-section model and the detector model. The listed errors are used for a penalty term computation, as discussed in Section 7.3. The first 16 parameters, indexed from 0 through 15, are used to adjust the neutrino flux, as discussed in Section 7.1.

defined in Sections 7.1 and 7.2. The differences between the data and MC simulation are measured using a $\chi^{2}$ function computed for several histograms. The $\chi^{2}$ function is defined as follows [107]:

$$
\chi^{2}\left(p_{1}, p_{2}, \ldots, p_{N_{p}}\right)=\sum_{i=1}^{N_{b i n}} \frac{\left(n_{D}^{i}-n_{M}^{i}\right)^{2}}{n_{D}^{i}+n_{M}^{i}}+\sum_{j=1}^{N_{P}} \frac{\left(p_{j}-p_{j}^{i n i t}\right)^{2}}{\sigma_{j}^{2}}
$$

where $N_{p}$ is the number of tuning parameters, $n_{D}^{i}$ is the number of data events in the bin $i$, and $n_{M}^{i}$ is the sum of MC event weights in the bin $i$. The second term is a penalty term, where $p_{j}$ is the tuning parameter $j, p_{j}^{\text {init }}$ is the initial (default) parameter value and $\sigma_{j}$ is the uncertainty of the parameter $j$. The histograms are binned in reconstructed event energy. The fit uses three (QES, RES, and DIS) histograms for the low energy beam and a single histogram for each additional beam configuration. The total $\chi^{2}$ is the sum over all histogram bins. The $\chi^{2}$ function depends on the flux and cross-section parameters via the MC event importance weights. The weights of data events are always set to unity. 
The fit is an iterative procedure consisting of these steps:

Step 1 Compute the MC event weights using the current tuning parameters.

Step 2 Fill the histograms with the MC and data events.

Step 3 Compute $\chi^{2}$ for the data and MC histograms.

Step 4 Adjust the tuning parameters and return to Step 1.

The fit minimizes the $\chi^{2}$ function by varying the flux and cross-section parameters. The fit is an iteration of multiple steps, where the parameters are gradually varied to find a global minimum of the $\chi^{2}$ function. This minimization step uses the MINUIT program [108].

\subsection{Kernel method and $\chi^{2}$ function}

The $\chi^{2}$ function is a different function of the energy scale and all other parameters, and this difference affects a minimization procedure. The flux and cross-section parameters change MC event weights, without changing reconstructed event energy. As a result, the $\chi^{2}$ function is a smooth function of these parameters, as illustrated in Figure 7.2. The energy scale parameters change reconstructed event energy. These parameters move events between different bins. The $\chi^{2}$ function is a discontinuous function of the energy scale parameter, as illustrated in Figure 7.2. A discontinuous $\chi^{2}$ function presents a problem for the MINUIT program because MINUIT searches for a minimum by following a gradient descent. The discontinuities in the $\chi^{2}$ function 
prevent MINUIT from computing reliable estimates for the first and second derivatives.

The discontinuity problem is solved using a kernel smoothing method [109]. This method introduces a finite event width so that one event can contribute some weight to multiple histogram bins. This method eliminates sudden changes in the $\chi^{2}$ function when events move between bins. Figure 7.2 shows the $\chi^{2}$ function plotted as a function of the energy scale parameters using the kernel smoothing method. This $\chi^{2}$ function does not have discontinuities. The kernel method does not change the position of the $\chi^{2}$ function minimum (within the statistical errors).
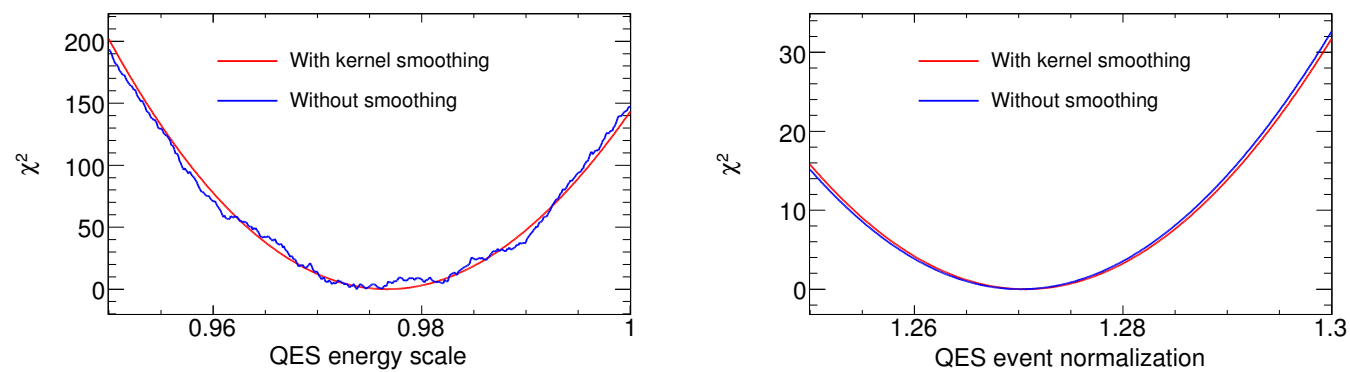

Figure 7.2: These figures show the $\chi^{2}$ function plotted as the function of the energy scale and QES event normalization parameters. The left plot uses the QES energy scale parameter. The right plot uses the QES event normalization parameter. The $\chi^{2}$ function shown in the red line uses the kernel smoothing method, as discussed in the text.

The kernel smoothing method uses the "kernel" function. This function is typically a narrow Gaussian distribution or a similarly shaped function [109]. 
The biweight kernel function is used for kernel smoothing in our analysis:

$$
K(t)= \begin{cases}\frac{15}{16}\left(1-t^{2}\right)^{2}, & \text { if }|t|<1 \\ 0, & \text { otherwise }\end{cases}
$$

The kernel width is set to $4 \%$ of the reconstructed event energy. This value is smaller than the energy resolution, but it is large enough to smooth the $\chi^{2}$ function.

Let $x_{E}$ denote the event energy and $w_{E}$ denote the event weight. Define the kernel width as $\sigma_{E}=0.04 \times x_{E}$. The event density is given by the following expression:

$$
K_{E}(x)= \begin{cases}\frac{15}{16} w_{E}\left(1-\frac{\left(x-x_{E}\right)^{2}}{\sigma_{E}^{2}}\right)^{2}, & \text { if }\left|x-x_{E}\right|<\sigma_{E}, \\ 0, & \text { otherwise. }\end{cases}
$$

The content of any histogram bin that has the non-zero overlap with this event is increased by $I_{w}$ :

$$
I_{w}=\int_{x_{L}}^{x_{H}} K_{E}(x) d x
$$

where $x_{L}$ is the lower bin edge and $x_{H}$ is the upper bin edge. The kernel method allows events to move smoothly between bins, thus avoiding discontinuities in the $\chi^{2}$ function. The histograms shown in this thesis do not include the smoothing procedure. The kernel smoothing is only used by the minimization procedure described in Section 7.3.

\subsection{Tuning results}

This section presents results of the near detector fits. The number of protons on target (POT) for the data and MC simulation is listed in Table 6.2. 
Tables 7.2 and 7.3 summarize the data and MC events used for two fits. A separate fit is performed for the LE data from the Run I and Run II periods. Differences between the two periods are discussed in Section 6.3. The data from other beam configurations are also included into the fits. Our analysis uses all reconstructed near detector data recorded before August of 2007.

The $\chi^{2}$ function for the Run I fit uses the 14 histogram pairs. ${ }^{5}$ The $\chi^{2}$ function for the Run II fit uses the 12 histogram pairs. Each pair corresponds to one line in Tables 7.2 and 7.3. These tables show the $\chi^{2}$ values and the number of events before (default MC) and after tuning (tuned MC). The difference in the number of events between the data and the MC simulation is measured using this variable:

$$
R^{\text {Tuned }}=\frac{N^{\text {Data }}-N^{\text {Tuned }}}{N^{\text {Data }}} \times 100 \%
$$

where $N^{\text {Data }}$ is the number of data events, and $N^{\text {Tuned }}$ is the number of MC events from the tuned MC simulation. The $R^{\text {Tuned }}$ values are listed in the last column of Tables 7.2 and 7.3. For the LE beam configuration, the number of tuned MC events agrees with the data within approximately $0.5 \%$. Overall, the tuned MC simulation accurately describes the near detector data for the two run periods, as discussed in Section 6.3.

\footnotetext{
${ }^{5}$ In each pair, one histogram is filled with the data events, and one histogram is filled with the MC events.
} 


\begin{tabular}{|c|c|c|c|c|c|c|c|c|c|}
\hline Beam type & Run period & & $\chi_{\text {start }}^{2}$ & $\chi_{\text {best }}^{2}$ & Ndof & $N^{\text {Data }}$ & $N^{\text {Default }}$ & $N^{\text {Tuned }}$ & $R^{\text {Tuned }}(\%)$ \\
\hline $\mathrm{L} 10 \mathrm{~cm} / 0 \mathrm{kA}$ & $1 \& 2$ & $\nu_{\mu}$ & 742.8 & 69.6 & 62 & 26966 & 21327 & 27297 & -1.23 \\
\hline $\mathrm{L} 10 \mathrm{~cm} / 170 \mathrm{kA}$ & 1 & $\nu_{\mu}$ & 250.6 & 36.0 & 62 & 14571 & 12711 & 14569 & 0.02 \\
\hline $\mathrm{L} 10 \mathrm{~cm} / 200 \mathrm{kA}$ & 1 & $\nu_{\mu}$ & 199.6 & 37.4 & 62 & 17009 & 15164 & 17009 & -0.00 \\
\hline $\mathrm{L} 100 \mathrm{~cm} / 200 \mathrm{kA}$ & 1 & $\nu_{\mu}$ & 212.4 & 96.1 & 62 & 23636 & 23533 & 24477 & -3.55 \\
\hline $\mathrm{L} 150 \mathrm{~cm} / 200 \mathrm{kA}$ & 2 & $\nu_{\mu}$ & 407.0 & 161.4 & 62 & 53611 & 53683 & 54289 & -1.26 \\
\hline $\mathrm{L} 250 \mathrm{~cm} / 200 \mathrm{kA}$ & 2 & $\nu_{\mu}$ & 1551.3 & 178.7 & 62 & 548359 & 561158 & 548928 & -0.10 \\
\hline $\mathrm{L} 10 \mathrm{~cm} / 185 \mathrm{kA}$ & 1 & $\nu_{\mu} \mathrm{DIS}$ & 13708.0 & 318.0 & 347 & 850088 & 730807 & 848036 & 0.24 \\
\hline $\mathrm{L} 10 \mathrm{~cm} / 185 \mathrm{kA}$ & 1 & $\nu_{\mu} \mathrm{QES}$ & 2508.7 & 179.7 & 96 & 187476 & 157214 & 187010 & 0.25 \\
\hline $\mathrm{L} 10 \mathrm{~cm} / 185 \mathrm{kA}$ & 1 & $\nu_{\mu} \mathrm{RES}$ & 1147.8 & 158.1 & 95 & 392348 & 369422 & 390647 & 0.43 \\
\hline $\mathrm{L} 10 \mathrm{~cm} / 0 \mathrm{kA}$ & $1 \& 2$ & $\bar{\nu}_{\mu}$ & 56.2 & 26.2 & 32 & 5965 & 5466 & 5715 & 4.18 \\
\hline $\mathrm{L} 10 \mathrm{~cm} / 185 \mathrm{kA}$ & 1 & $\bar{\nu}_{\mu}$ & 574.5 & 67.7 & 62 & 124428 & 117063 & 124303 & 0.10 \\
\hline $\mathrm{L} 100 \mathrm{~cm} / 200 \mathrm{kA}$ & 1 & $\bar{\nu}_{\mu}$ & 23.3 & 21.7 & 32 & 1005 & 1025 & 1077 & -7.25 \\
\hline $\mathrm{L} 150 \mathrm{~cm} / 200 \mathrm{kA}$ & 2 & $\bar{\nu}_{\mu}$ & 23.7 & 23.7 & 32 & 1904 & 1937 & 2040 & -7.11 \\
\hline $\mathrm{L} 250 \mathrm{~cm} / 200 \mathrm{kA}$ & 2 & $\bar{\nu}_{\mu}$ & 29.0 & 34.1 & 32 & 16565 & 16045 & 16762 & -1.18 \\
\hline & & & 21435.0 & 1408.5 & 1100 & & & & \\
\hline
\end{tabular}

Table 7.2: This table lists the beam configuration, the selection type, and the number of histogram bins. The table also lists the $\chi^{2}$ values and the number of events in the data and MC simulation, before and after the fit. This fit includes all of the Run I LE data in addition to the data from other beam configurations. 


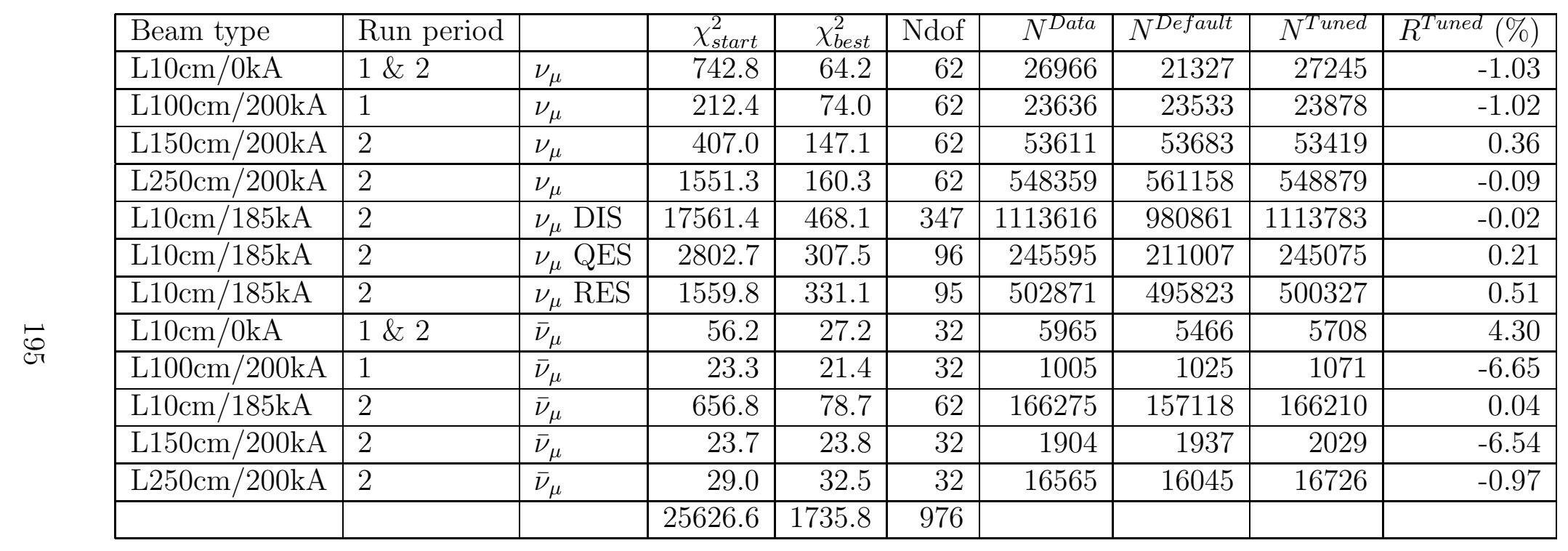

Table 7.3: This table lists the beam configuration, the selection type, and the number of histogram bins. The table also lists the $\chi^{2}$ values and the number of events in the data and MC simulation, before and after the fit. This fit includes all of the Run II LE data in addition to the data from other beam configurations. 
Table 7.4 lists the tuning parameters that minimize the $\chi^{2}$ function (best fit point), for the Run I and Run II fits. The results for the two periods agree within the statistical and systematic errors. The statistical error is computed by varying each parameter (one parameter at a time) near the best fit

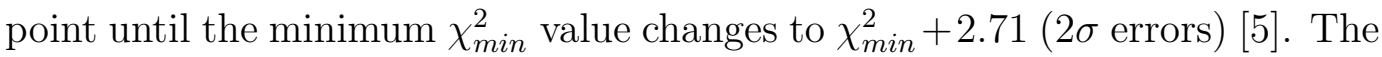
systematic parameters are determined by changing the selection requirements, as discussed in Section 6.2. A total of nine additional fits are performed for both the Run I and Run II data, where each fit uses events selected with a different set of the selection requirements. The systematic errors are computed as the maximum deviation between the best fit parameters obtained using the primary selection method and the best fit parameters obtained using the nine additional sets. Additional systematic errors are discussed in Section 7.6.

Parameters $p_{0}$ through $p_{15}$ change the yield of the neutrino hadron parents as a function of the transverse, $p_{T}$, and longitudinal, $p_{z}$, momentum components. Figure 7.3 shows the weight of the $\pi^{ \pm}$neutrino parents for the Run I and Run II fits. The tuning procedure increases the number of $\pi^{+}$ parents in the forward direction for $p_{T}<50 \mathrm{MeV}$. The fit increases the total number of $\pi^{ \pm}$and $K^{ \pm}$hadrons produced by the NuMI target.

Parameters $p_{16}$ through $p_{21}$ adjust the cross-section model and the energy scale for the $\nu_{\mu}$ charged-current and $\bar{\nu}_{\mu}$ charged-current events. The four energy scales are decreased by approximately $2 \%$. This result suggests that the overall detector energy scale is off by approximately $2 \%$, which is within the $5 \%$ uncertainty of the detector energy scale. This result also implies that the 

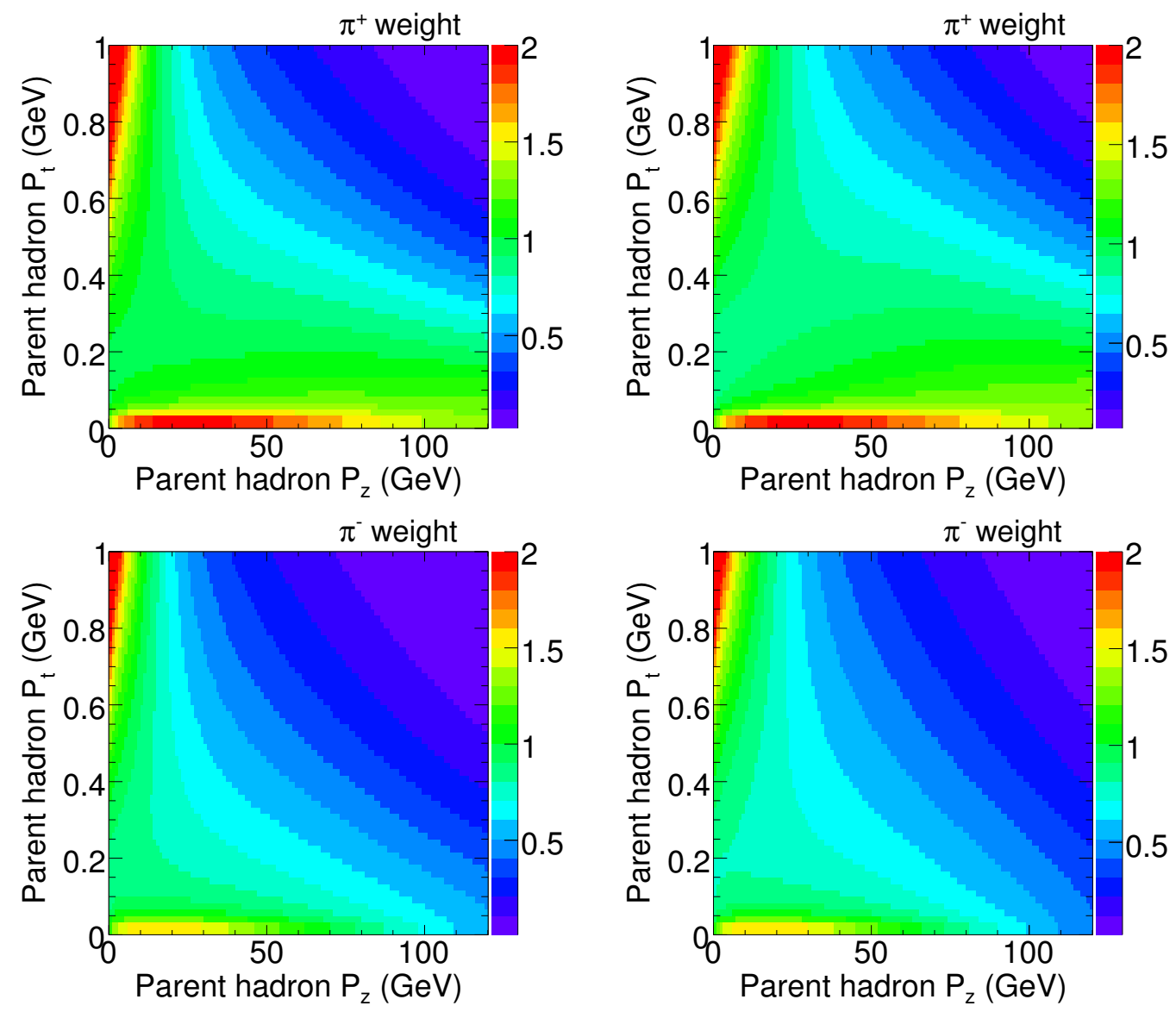

Figure 7.3: The weight of the $\pi^{+}$and $\pi^{-}$neutrino parents is plotted as a function of the $p_{T}$ and $p_{z}$ momentum components. These figures show the weights obtained with the best fit parameters from Table 7.4. The left (right) figures show weights for the Run I (Run II) fit. 


\begin{tabular}{ll|lll|lll} 
Par. & & Run I & Stat. & Syst. & Run II & Stat. & Syst \\
\hline 0 & $\pi^{+}$ & -0.718 & \pm 0.019 & \pm 0.394 & -0.861 & \pm 0.017 & \pm 0.327 \\
1 & $\pi^{+}$ & -1.167 & \pm 0.080 & \pm 0.027 & -1.237 & \pm 0.071 & \pm 0.026 \\
2 & $\pi^{+}$ & 0.8013 & \pm 0.0018 & \pm 0.1062 & 0.6997 & \pm 0.0015 & \pm 0.0846 \\
3 & $\pi^{+}$ & 0.541 & \pm 0.010 & \pm 0.017 & 0.7122 & \pm 0.0089 & \pm 0.0066 \\
4 & $\pi^{+}$ & 0.7499 & \pm 0.0032 & \pm 0.1339 & 0.7397 & \pm 0.0030 & \pm 0.1238 \\
5 & $\pi^{+}$ & 1.727 & \pm 0.024 & \pm 0.057 & 1.307 & \pm 0.023 & \pm 0.070 \\
\hline 6 & $K^{+}$ & 1.11 & \pm 0.13 & \pm 0.02 & 1.11 & \pm 0.11 & \pm 0.01 \\
7 & $K^{+}$ & 0.00 & \pm 0.24 & \pm 0.01 & -0.01 & \pm 0.23 & \pm 0.02 \\
8 & $K^{+}$ & 1.0147 & \pm 0.0071 & \pm 0.0694 & 0.9635 & \pm 0.0064 & \pm 0.0514 \\
9 & $K^{+}$ & 0.407 & \pm 0.017 & \pm 0.096 & 0.504 & \pm 0.015 & \pm 0.068 \\
10 & $K^{+}$ & 0.588 & \pm 0.016 & \pm 0.054 & 0.551 & \pm 0.015 & \pm 0.037 \\
11 & $K^{+}$ & 1.464 & \pm 0.048 & \pm 0.012 & 1.506 & \pm 0.045 & \pm 0.014 \\
\hline 12 & $\pi^{-}$ & 0.8885 & \pm 0.0070 & \pm 0.0224 & 0.9290 & \pm 0.0062 & \pm 0.0203 \\
13 & $\pi^{-}$ & -0.479 & \pm 0.021 & \pm 0.056 & -0.617 & \pm 0.019 & \pm 0.061 \\
\hline 14 & $K^{-}$ & 0.811 & \pm 0.041 & \pm 0.015 & 0.894 & \pm 0.036 & \pm 0.015 \\
15 & $K^{-}$ & 0.08 & \pm 0.10 & \pm 0.03 & 0.054 & \pm 0.090 & \pm 0.046 \\
\hline 16 & $E_{\bar{\nu}}$ & 0.9871 & \pm 0.0040 & \pm 0.0047 & 0.9886 & \pm 0.0036 & \pm 0.0038 \\
17 & $E_{\nu}^{D I S}$ & 0.9822 & \pm 0.0012 & \pm 0.0028 & 0.9822 & \pm 0.0011 & \pm 0.0025 \\
18 & $E_{\nu}^{Q E S}$ & 0.9787 & \pm 0.0026 & \pm 0.0147 & 0.9651 & \pm 0.0022 & \pm 0.0138 \\
19 & $N_{\nu}^{Q E S}$ & 1.2311 & \pm 0.0078 & \pm 0.0395 & 1.2929 & \pm 0.0072 & \pm 0.0345 \\
20 & $E_{\nu}^{R E S}$ & 0.9846 & \pm 0.0027 & \pm 0.0038 & 0.9703 & \pm 0.0025 & \pm 0.0042 \\
21 & $N_{\nu}^{R E S}$ & 0.8768 & \pm 0.0063 & \pm 0.0641 & 0.8626 & \pm 0.0057 & \pm 0.0549
\end{tabular}

Table 7.4: Best fit parameters for the Run I and Run II fits using the data listed in Tables 7.2 and 7.3. The parameters are defined in Table 7.1 and in Equations 7.7 and 7.8 . 
systematic error for the energy scale is independent from the hadronic shower energy. In these fits, the energy of the selected QES events is computed using Equation 3.5.

The number of true QES events is scaled up by approximately $25 \%$. The number of true RES events is scaled down by approximately $13 \%$. The additional systematic errors for these two parameters are discussed in Section 7.6. Figures 6.11 and 6.13 show the reconstructed energy for the selected QES, RES, and DIS events for the data and tuned MC simulation. This result undescores the earlier statement that the simple scale parameters for the QES and RES events accurately describe the data.

\subsection{Study of different fit approaches}

The fit parameters listed in Table 7.4 are the corrections for the MC simulation. These values depend on the neutrino flux and cross-sections models used by the MC simulation. To illustrate this point, the near detector data set was analyzed using two versions of the MC simulation: current and previous. There were two major changes applied to the current MC simulation, compared with the previous version:

1. The secondary pions are produced in interaction of neutrinos. These pions can interact with a nucleus (final-state interactions). The average interaction probability of the secondary pions with the nucleus was increased by approximately $8 \%[110,111]$. However, the neutrino cross- 
sections were unchanged.

2. The current MC simulation uses the new magnetic field map, described in Section 6.1. The new map has a 12\% stronger magnetic field than the old map, used by the previous MC simulation.

The complete tuning procedure was performed using the near detector Run I data and these two MC simulations. The fit results for the 6 fit parameters are presented in Table 7.5. The flux parameters are not shown for clarity, these parameters have similar values for the two MC versions. For the previous MC version, the QES scale factor is 1.48 compared to 1.23 for the current MC version. This result indicates that the QES scale factor adjusts for the nuclear effects in the MC simulation. These nuclear effects include the pion absorption, which reduces the visible hadronic energy in the detector. As a result, the nuclear effects scramble the signature of the RES events. The values of the QES and RES scale parameters are consistent with this observation. For the previous MC version, the RES scale factor is 0.98 compared to 0.88 for the current $\mathrm{MC}$ version.

The same magnetic field map is used to reconstruct the muon momentum via the curvature for the data and MC tracks. The MC simulation also uses the magnetic field map to simulate the passage of muons through the detector. The MC simulation consistently uses the single magnetic field map for the simulation and reconstruction steps. The data muons sample the real magnetic field in the detector, which could be different from the magnetic field 


\begin{tabular}{ll|ll|ll} 
Par. & & Curr. MC & Stat. & Prev. MC & Stat. \\
\hline 16 & $E_{\bar{\nu}}$ & 0.9871 & \pm 0.0040 & 0.9682 & \pm 0.0039 \\
17 & $E_{\nu}^{D I S}$ & 0.9822 & \pm 0.0012 & 0.9642 & \pm 0.0012 \\
18 & $E_{\nu}^{Q E S}$ & 0.9787 & \pm 0.0026 & 0.9596 & \pm 0.0023 \\
19 & $N_{\nu}^{Q E S}$ & 1.2311 & \pm 0.0078 & 1.4755 & \pm 0.0086 \\
20 & $E_{\nu}^{R E S}$ & 0.9846 & \pm 0.0027 & 0.9795 & \pm 0.0026 \\
21 & $N_{\nu}^{R E S}$ & 0.8768 & \pm 0.0063 & 0.9837 & \pm 0.0068
\end{tabular}

Table 7.5: Best fit parameters for the Run I data obtained using the current and previous versions of the MC simulation and event reconstruction software. For clarity of presentation, only 6 parameters are shown. These parameters adjust the cross-section and reconstructed energy scales.

map used for the reconstruction (within the systematic uncertainties). If the incorrect magnetic field map is used to reconstruct the data tracks, then the average muon momentum from the curvature is shifted from its real value. For the previous MC version, the energy scale parameters were consistently lower than the parameters obtained with the current MC version. This fact suggests that the energy scale parameters are correcting for the wrong muon momentum via the curvature in the data. This observation was confirmed by fitting directly for the scale of the muon momentum from the curvature. In conclusion, out fit procedure has a real sensitivity to the detector energy scale.

The second study compares the two energy reconstruction methods described in Section 3.4. The current MC version was used for this study. The $E_{\nu}$ formula (defined in Equation 3.1) and $E_{Q E S}$ formula (defined in Equation 3.5) are used to reconstruct the energy of the selected QES events in the data and MC simulation. The $E_{Q E S}$ formula uses the reconstructed muon angle to estimate the missing hadronic energy in the selected QES events, as discussed in 


\begin{tabular}{ll|ll|ll} 
Par. & & $E_{Q E S}$ & Stat. & $E_{\nu}$ & Stat. \\
\hline 16 & $E_{\bar{\nu}}$ & 0.9871 & \pm 0.0040 & 0.9877 & \pm 0.0040 \\
17 & $E_{\nu}^{D I S}$ & 0.9822 & \pm 0.0012 & 0.9814 & \pm 0.0012 \\
18 & $E_{\nu}^{Q E S}$ & 0.9787 & \pm 0.0026 & 0.9733 & \pm 0.0026 \\
19 & $N_{\nu}^{Q E S}$ & 1.2311 & \pm 0.0078 & 1.2306 & \pm 0.0078 \\
20 & $E_{\nu}^{R E S}$ & 0.9846 & \pm 0.0027 & 0.9881 & \pm 0.0027 \\
21 & $N_{\nu}^{R E S}$ & 0.8768 & \pm 0.0063 & 0.8791 & \pm 0.0063
\end{tabular}

Table 7.6: Best fit parameters for the Run I data. $E_{Q E S}$ column uses the $E_{Q E S}$ formula from Equation 3.5 to reconstruct energy for the selected QES events. $E_{m u}$ column uses the $E_{\nu}$ formula from Equation 3.1 to reconstruct energy for the selected QES events.

Section 3.4. The hadronic energy is missing in the QES events, when the final state proton is absorbed within the nucleus or stopped in the steel. Table 7.6 compares the fit parameters obtained in these two fits. The first fit uses the $E_{\nu}$ formula, and the second fit uses $E_{Q E S}$ formula. The QES energy scale parameters for the two methods agree to within $0.5 \%$. This difference suggests the size of the unmodelled effects present in the data, which can change the amount of visible hadronic energy in the QES events.

\subsection{Summary}

In this chapter, the average event rate of the QES and RES events was adjusted by $1.26 \pm 0.04$ and $0.87 \pm 0.06$, respectively. These corrections are applied relative the QES and RES cross-section models in the NEUGEN event generator. The systematic errors from the nuclear effects were estimated: approximately \pm 0.24 for the QES events, and approximately \pm 0.11 for the RES events. Further work is required to understand the nuclear effects. It is 
not clear that the described procedure fully accounts for all the uncertainties from the final state interactions. The detector energy scale was adjusted by approximately $2 \%$, within the estimated $5 \%$ uncertainty.

There are substantial differences between the MINOS near detector data and MC simulation. These differences are most likely caused by the uncertainties in modeling the neutrino flux and cross-sections. The MC simulation is tuned using 22 parameters. These parameters adjust the neutrino flux and cross-sections models. The tuned MC simulation accurately describes the near detector data, as shown in Chapter 6. This tuned MC simulation is used to generated the predicted far detector events for the analysis of the neutrino oscillations in Chapter 9. 


\section{Chapter 8}

\section{Muon neutrino events in the far detector}

The MINOS experiment measures neutrino oscillation parameters by comparing the rate of the $\nu_{\mu}$ charged-current interactions at the near and far detectors. A measurement of the neutrino events in the near detector is used to predict the expected event rate as a function of energy at the far detector. A fraction of muon neutrinos oscillates between the near and far detectors, resulting in an energy dependent deficit of the observed far detector $\nu_{\mu}$ charged-current events. This chapter discusses the differences in the neutrino interaction rate between the near and far detectors. Then, the selection procedure for the far detector events is summarized. The selected $\nu_{\mu}$ charged-current events in the far detector are examined by comparing these events with the expected event distributions. The oscillation analysis of the far detector events will be presented in Chapter 9.

\subsection{Extrapolation from near to far detectors}

The far detector is located $734.3 \mathrm{~km}$ away from the near detector. All neutrinos reaching the far detector also pass through the near detector. The near detector data is used to predict the number of neutrinos reaching the 
far detector. The distance and the direction from the neutrino source to the detector determine the rate of neutrino interactions and predict differences between the two detectors. This section briefly describes the computation of the predicted event rate at the detector.

In the NuMI neutrino beam, the majority of muon neutrinos are produced in the two-body pion and kaon decays; three body kaon decays contribute less than $0.1 \%$ to the $\nu_{\mu}$ flux. The neutrino energy is determined by the kinematics of the two-body decay in a lab frame and the trajectory of the neutrino to the detector. For the two-body $\pi \rightarrow \mu \nu$ and $K \rightarrow \mu \nu$ decays of the neutrino hadron parents, the neutrino energy is given by this expression:

$$
E_{\nu} \approx \frac{\left(1-\frac{m_{\mu}^{2}}{M^{2}}\right) E}{1+\gamma^{2} \tan ^{2} \theta_{\nu}}
$$

where $m_{\mu}$ and $M$ are the muon and hadron masses, E is the hadron energy, $\gamma=E / M$ is the hadron's Lorentz boost in the lab frame, and $\theta_{\nu}$ is the angle between the neutrino and parent hadron directions in the lab frame. A probability that the neutrino decays in a particular direction is given by the expression:

$$
\frac{d P}{d \Omega_{\nu}} \approx \frac{1}{4 \pi} \frac{4 \gamma^{2}\left(1+\tan ^{2} \theta_{\nu}\right)^{3 / 2}}{\left(1+\gamma^{2} \tan ^{2} \theta_{\nu}\right)^{2}}
$$

The far detector occupies a small solid angle, as viewed from the position of the NuMI target at Fermilab. Figure 8.1 illustrates the geometrical differences between the two detectors. The geometry of the decay pipe and the distance to the two detectors are included in the MC simulation. The MC 


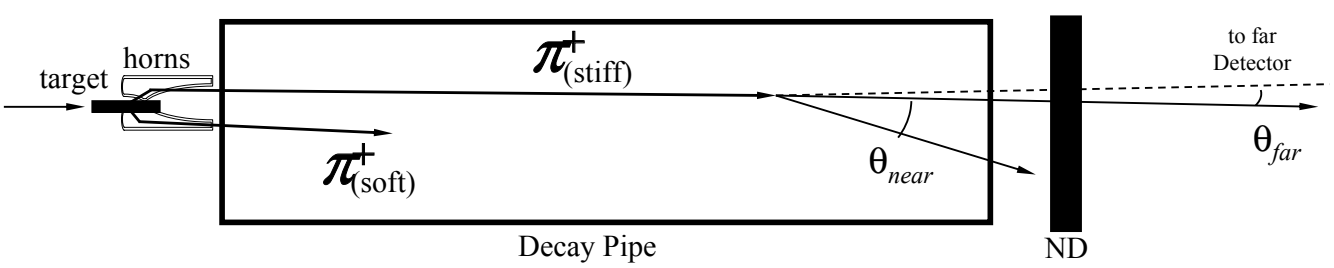

(a)

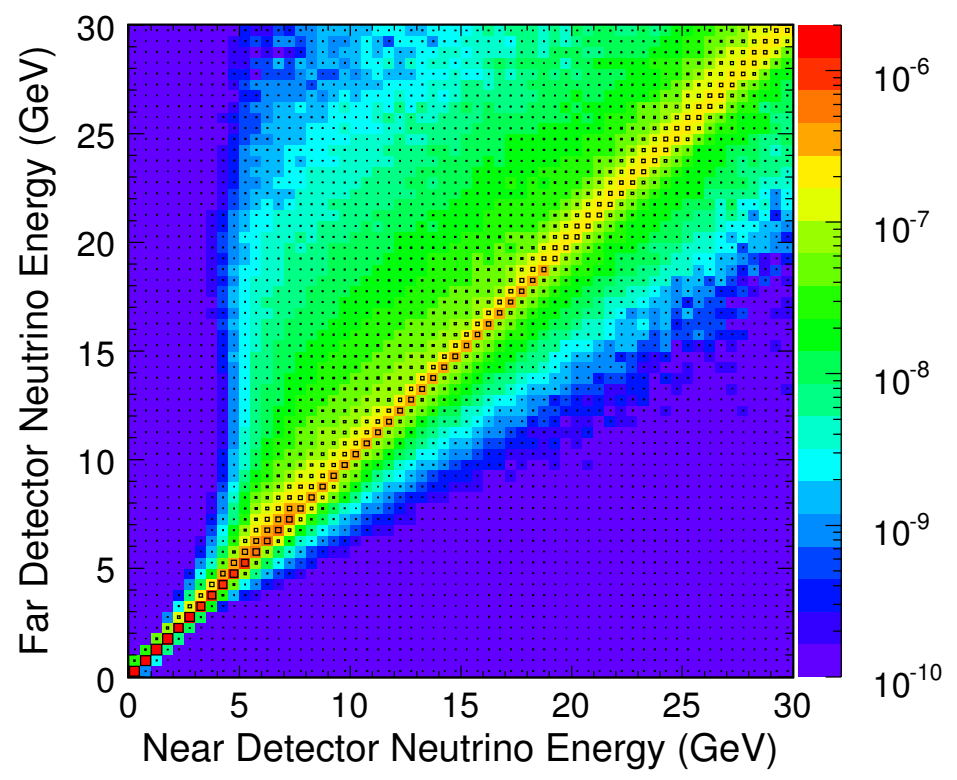

(b)

Figure 8.1: Figure (a) illustrates the differences between the near and far detectors for the $\nu_{\mu}$ charged-current interaction rates at the two detectors. These differences are due to the angular acceptance effects and the distances between the decay point to the two detectors. Figure (b) shows the join distribution of neutrino energies observed in the near and far detectors. The content of each cell represents the mean number of $\nu_{\mu}$ charged-current events expected at the far detector for one $\nu_{\mu}$ charged-current event in the near detector. These figures were taken from [58]. 
simulation predicts the rate of $\nu_{\mu}$ charged-current interactions at the far detector, which is about $1.4 \times 10^{-6}$ smaller than the rate of the $\nu_{\mu}$ charged-current interactions at the near detector.

There are two general approaches used by the MINOS collaboration to compute the rate of the far detector events [58]. Direct methods use a transfer matrix, computed using the MC simulation of the two detectors. This matrix directly transports the event rate as a function of the reconstructed energy from the near detector to the far detector, as illustrated in Figure 8.1b. Tuning (fitting) methods use the near detector data to adjust the parameters of the MC simulation. The tuned MC simulation is then used to compute the far detector event rate. The difference between the near detector data and the tuned MC simulation estimates the accuracy of the tuning procedure. The present analysis employs the tuning method, described in Chapter 7. Figure 8.3 compares the predicted event rate at the far detector without neutrino oscillation for the present analysis and for the recent MINOS analysis [46]; the later result used the direct extrapolation method. There is a discrepancy between two approaches for $E_{\nu}<1 \mathrm{GeV}$. This is consistent with the residual disagreements between the near detector data and the MC simulation for our analysis, as shown in Chapter 6. See Chapter 9, for additional comparisons of the two methods.

There are two significant uncertainties in predicting the neutrino interaction rate at the near and far detectors. The uncertainty for the production of pions and kaons is discussed in Chapter 7. In addition, there are uncertainties 


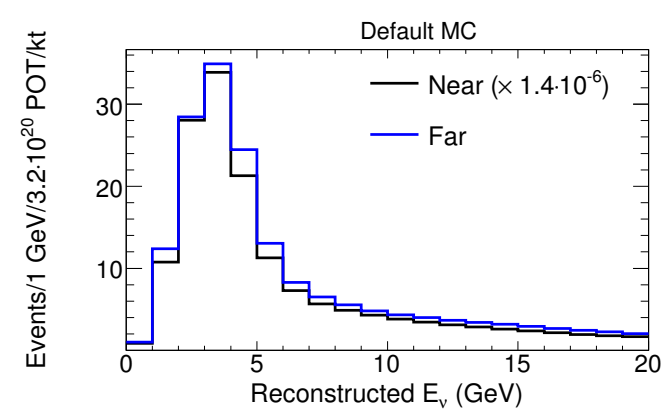

(a)

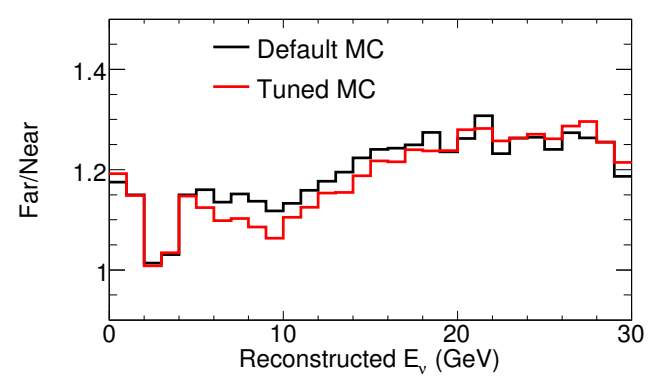

(c)

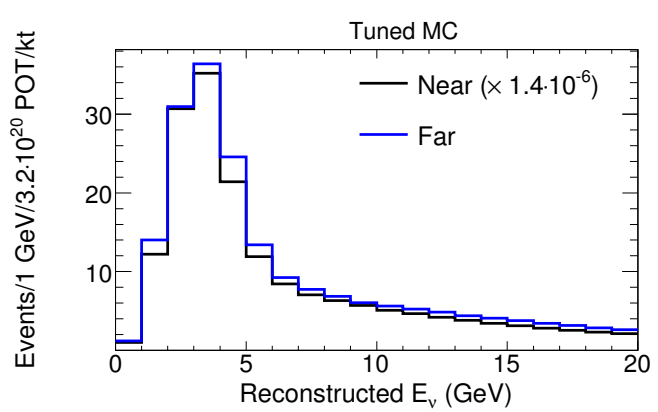

(b)

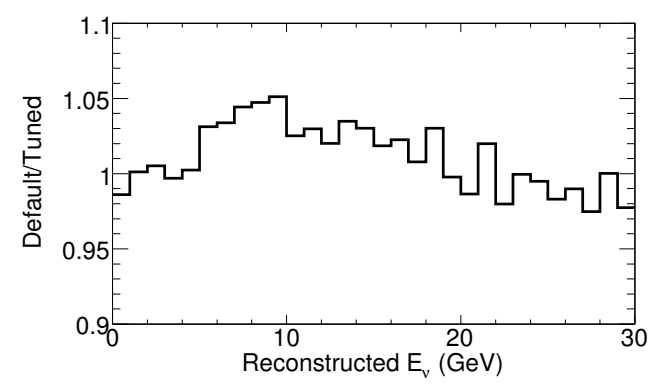

(d)

Figure 8.2: These figures show MC events from the near and far detectors, normalized by the number of POT and the fiducial mass. These figures show the spectrum for the default (a) and tuned (b) MC simulations. Figure (c) shows the ratio of the far over near detector events $\left(\times 1.4 \cdot 10^{-6}\right)$ for the default and tuned MC simulation. Figure (d) shows the ratio of the two histograms from Figure (c).
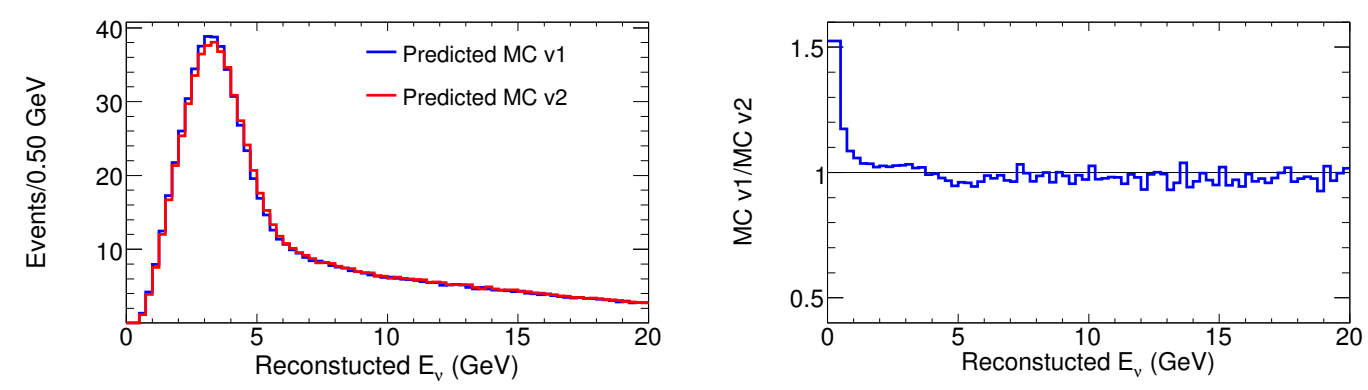

Figure 8.3: Predicted MC events at the far detector. This figure compares the present analysis (MC v1) and the recent MINOS analysis [46] (MC v2) using the identically selected $\nu_{\mu}$ charged-current events. 
associated with the measurements of the properties of beamline elements, such as the position and orientation of the NuMI target and horns. These uncertainties were studied using the MC simulation of the beamline elements. The errors on the predicted far detector event rate were determined to be less than $3 \%$, if the near detector data were used to compute this predicted rate [101].

Figure 8.2 shows the energy spectra for the selected $\nu_{\mu}$ charged-current $\mathrm{MC}$ events at the near and far detectors. The difference between the default and tuned MC simulations for the double ratio of the far over near energy spectra is less than $5 \%$. This fact illustrates the key feature of a two-detector oscillation experiment: the uncertainty of the predicted far detector event rate is reduced if the near detector data are used to compute the event rate.

\subsection{Selecting far detector events}

The far detector events are selected using the expected arrival time of the neutrino beam spills (see Section 2.3). The periods in which the far detector was in a "bad" state (e.g., the magnet coil was off, problems with GPS, high voltage was off) were excluded from the analysis. Figure 8.4 shows the timing distribution of the far detector events relative to the arrival time of the nearest neutrino beam spill. Using the GPS clocks, the MINOS collaboration measured the speed of muon neutrinos traveling from Fermilab to the far detector; the muon neutrino speed is consistent with the speed of light [112].

The far detector events were selected from the Run I and Run II periods using the low energy beam configuration. The Run I period accumulated 

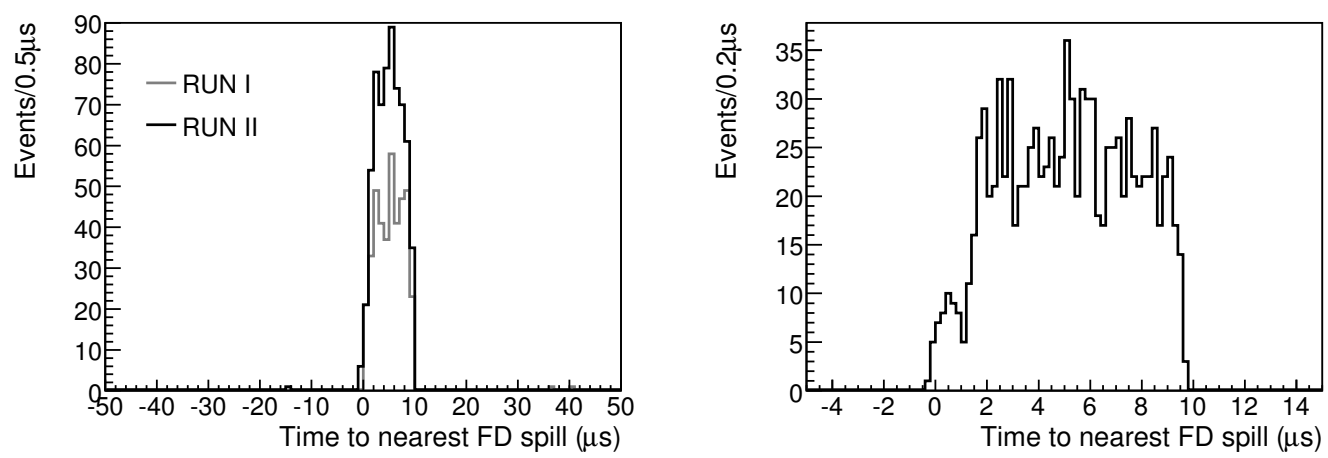

Figure 8.4: Timing of the far detector events, relative to the arrival time of the nearest beam spill. The left plot shows separately events from Run I and Run II. The right plot shows all events. Visible in the right plot is the batch structure of the primary proton beam in the Main Injector.

$1.27 \times 10^{20}$ protons on target. The Run II period accumulated $1.94 \times 10^{20}$ protons on target. The two periods were separated by the accelerator shutdown, as summarized in Section 6.3. The data from each run period are analyzed separately.

The far detector events, within a $50 \mu s$ timing window of the neutrino spill time, are subject to additional analysis cuts. The events are required to have a reconstructed track. The vertex of this track is required to be located within the fiducial volume. ${ }^{1}$ The fiducial volume consists of 448 detector planes, from planes 4 to 239 and 253 to 454 inclusive, and the radius between $0.4 \mathrm{~m}$ and $\sqrt{14} m \approx 3.74 \mathrm{~m}$. The far detector fiducial mass is $4.17 \mathrm{kt}$. The $\nu_{\mu}$ charged-current interactions are selected using the muon selection algorithm described in Chapter 4. The four track variables used for the muon identifi-

\footnotetext{
${ }^{1}$ The longest track is used if more than one track is present in the event.
} 

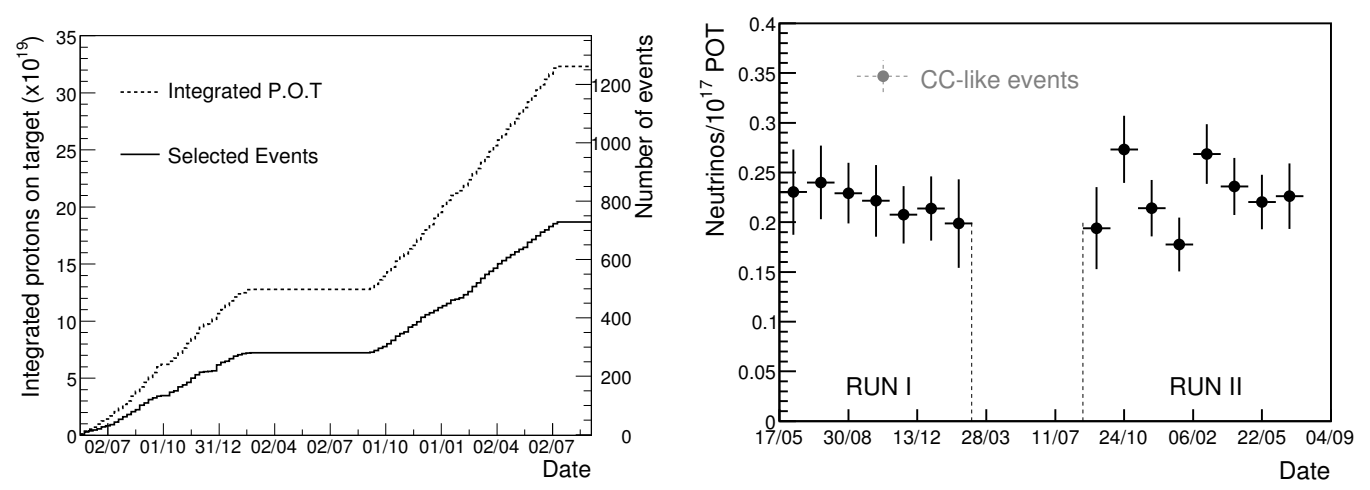

Figure 8.5: The left plots shows both the number of the far detector events and the number of the accumulated protons on target as a function of time. The right plots shows the number of the far detector events per $10^{17}$ protons as a function of time.

cation and the discriminant variable, $P_{S}$, are shown in Figure 8.6. The muon charge sign selection is applied in order to select the $\nu_{\mu}$ charged-current events, as described in Chapter 5.

The direction of the reconstructed track at the vertex is required to be within $53.1^{\circ}$ angle with the detector $\mathrm{Z}$ axis. This requirement was implemented to minimize the number of background events induced by cosmic ray muons. A number of these background events were estimated using two methods. First, the rate of events outside the actual $10 \mu \mathrm{s}$ neutrino beam spill window was examined. Second, the far detector data recorded in anti-coincidence with the beam spills were analyzed using the selection criteria described above. The two methods predicted less than 0.5 background events for the Run I data [58]. This background is significantly smaller than the $4 \%$ uncertainty on the absolute normalization of the predicted far detector event rate. 

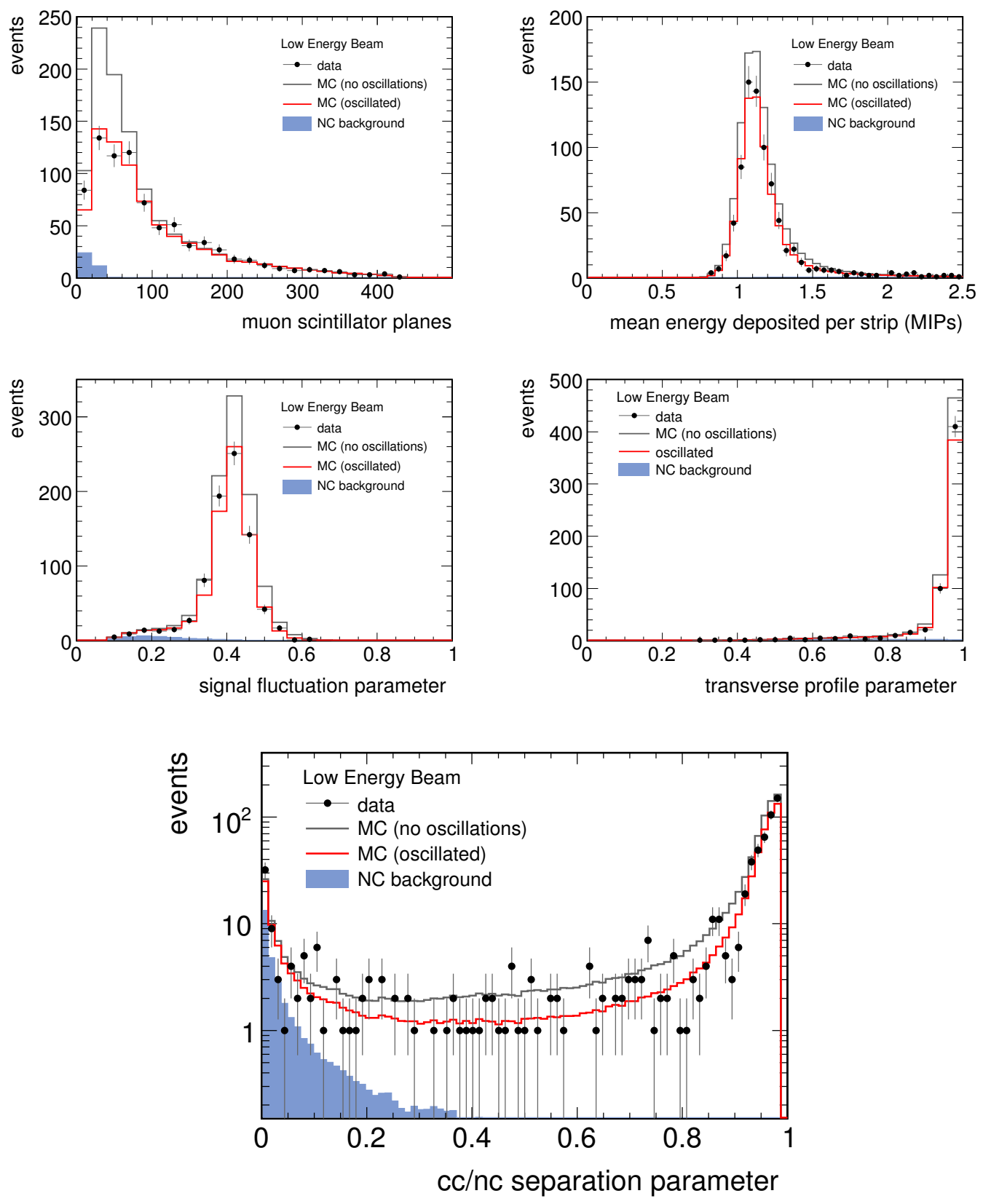

Figure 8.6: The top four figures show the four input variables used for the muon identification. The bottom figure shows the event classification parameter. The data events are compared to the MC events in absence of oscillations and the MC events with generic oscillation parameters. Both versions of the MC simulation are adjusted using the near detector data and the matrix method [46]. 


\subsection{Far detector data}

The MINOS collaboration follows a blind analysis policy for the far detector events produced by the NuMI neutrino beam. This policy was implemented to minimize a potential physicist bias. All analysis techniques were developed using MC events and near detector data; beam neutrino events in the far detector were not used. The far detector data events were revealed in steps, where a subset of all events was examined first. Then, simple event distributions were examined for the entire data set to check for any potential problems $[46,52,58]$. The present analysis uses the subset of the $\nu_{\mu}$ charged-

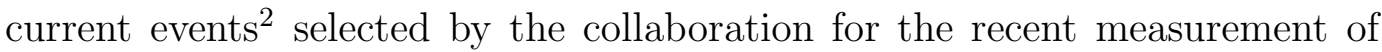
the muon neutrino disappearance [46].

In this chapter, the selected $\nu_{\mu}$ charged-current far detector events are compared with the "predicted" and "oscillated" MC events. The predicted MC events are generated assuming no neutrino oscillations. These MC events use the tuned MC simulation, obtained from the default MC events by applying the flux and cross-section corrections derived in Chapter 7 . The oscillated MC events are obtained from the predicted MC events by applying the twoflavor oscillation hypothesis; the predicted MC events are "weighted" using the oscillation parameters measured in Chapter 9. The MC events are scaled to the number of POT in the Run I and Run II data. In this section, the figures show distributions for the sum of the Run I and Run II events.

\footnotetext{
${ }^{2}$ The list of events for the published MINOS analysis was provided by David Petyt. Our analysis has applied a more restrictive charge sign requirement.
} 
Figure 8.7 shows the positions of the track vertex and the track end for the selected $\nu_{\mu}$ charged-current events. The distributions of the track vertexes is uniform throughout the detector. The selected $\left(\mu^{-}\right)$tracks tend to stop in the coil. This effect is seen as an increase in the number of tracks that end within a small radius from the coil (located at $R=0 \mathrm{~m}$ ). A fraction of tracks leave the detector through the last plane. Figure 8.8 shows the number of event scintillator strips and the event pulse height. The effect of muon neutrino disappearance is seen as a depletion of the short events in the data. Figures 8.9 shows the reconstructed muon and shower energy as well as the total event energy. Figure 8.10 shows the kinematic variables.

In these figures, the oscillation hypothesis provides an accurate description of the data. The distributions of the track vertex positions are independent from the neutrino energy. As a result, the oscillation effect only scales down the entire distribution, uniformly throughout the detector. The muon neutrino disappearance is maximum around $1.5 \mathrm{GeV}$ of the true neutrino energy. This produces a depletion of the low energy events, as observed in the event length and pulse height distributions. The shape of the reconstructed energy spectrum in Figure 8.9 is the most clear evidence for the disappearance of the muon neutrino at the far detector. 

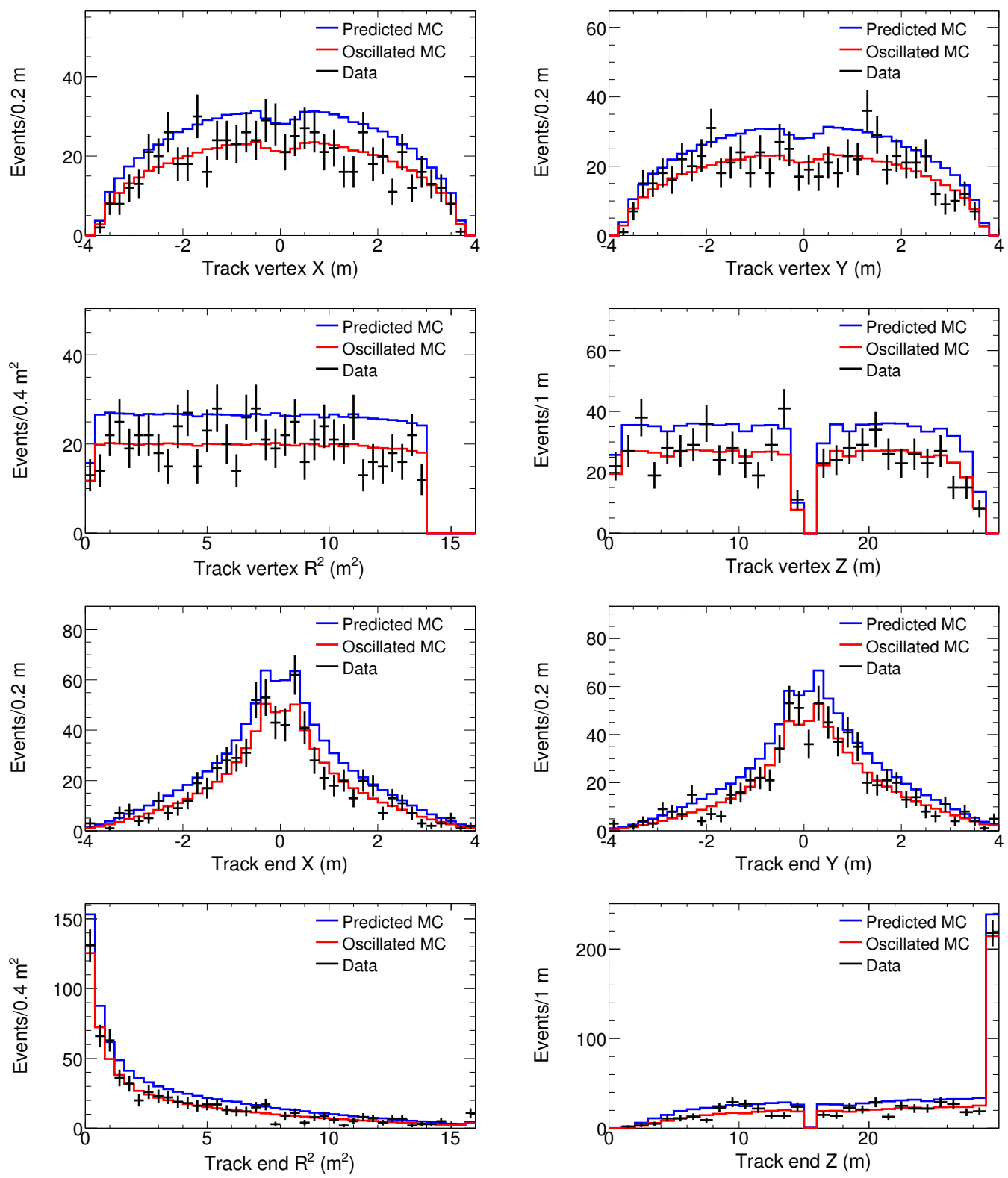

Figure 8.7: These figures show the positions of the track vertex and track end for the selected $\nu_{\mu}$ charged-current far detector events. The data events are compared to the predicted MC events (no oscillations) and the oscillated MC events, as discussed in the text. 

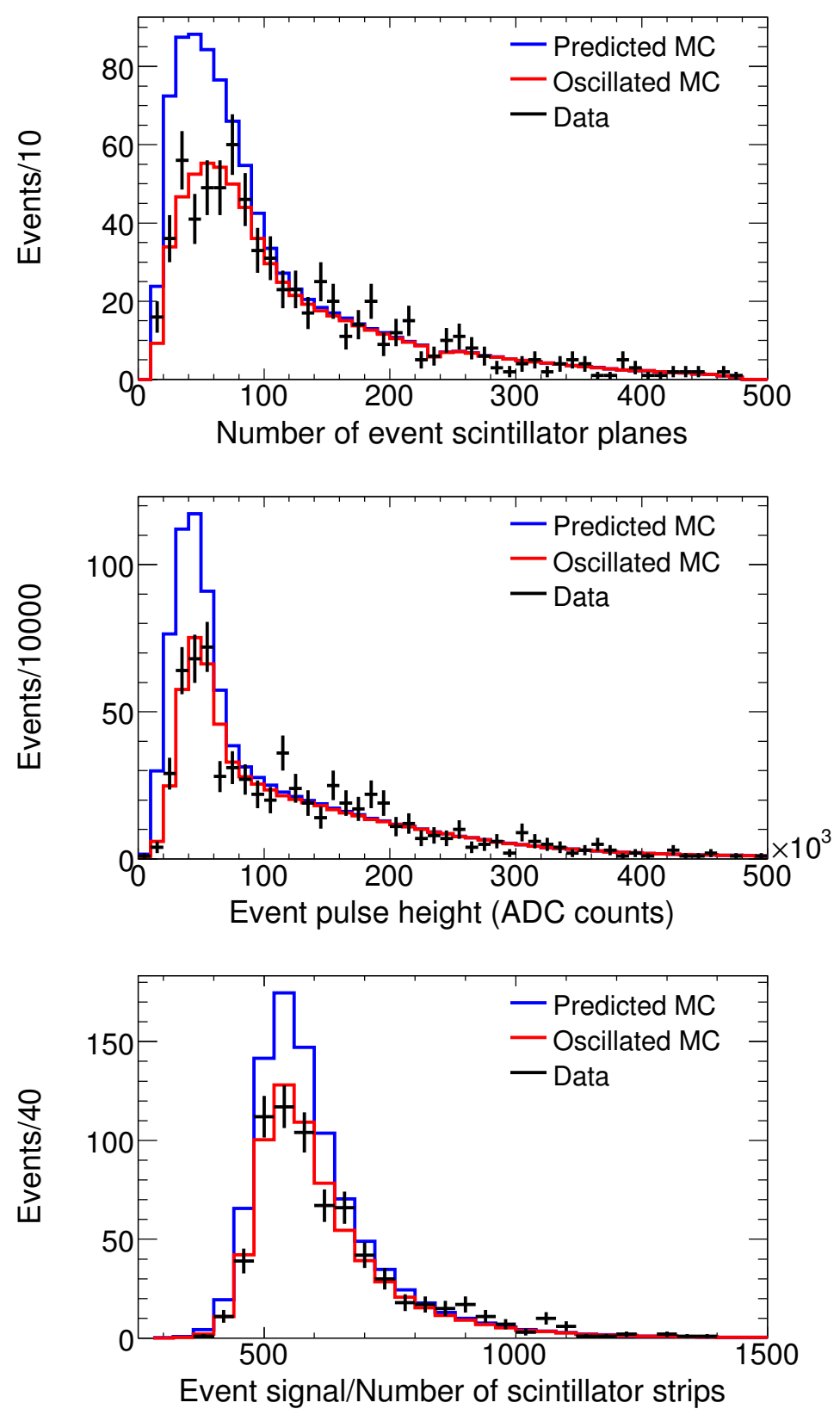

Figure 8.8: These figures show the selected $\nu_{\mu}$ charged-current far detector events. The top figure shows a number of event scintillator planes. The middle figure shows event pulse height, measured in the ADC units, where 100 ADC $\approx 1$ photoelectron. The bottom figure shows the event pulse height divided by the number of event scintillator strips. 

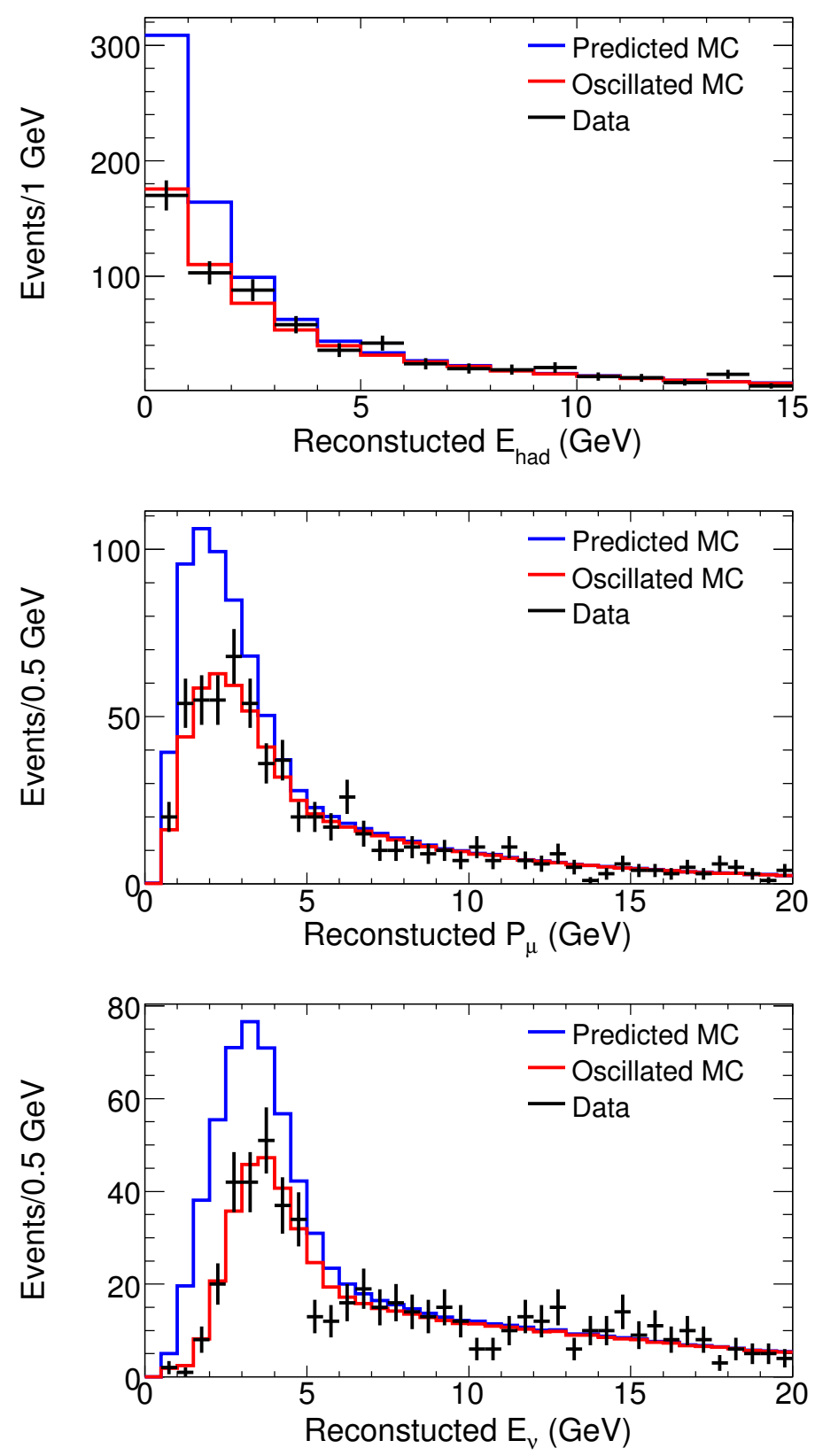

Figure 8.9: These figures show the selected $\nu_{\mu}$ charged-current far detector events. The top figure shows the reconstructed hadronic shower energy, $E_{\text {had }}$. The middle figure shows the reconstructed muon momentum, $P_{\mu}$. The bottom figure shows the total event energy, $E_{\nu}=E_{\text {had }}+E_{\mu}$. 

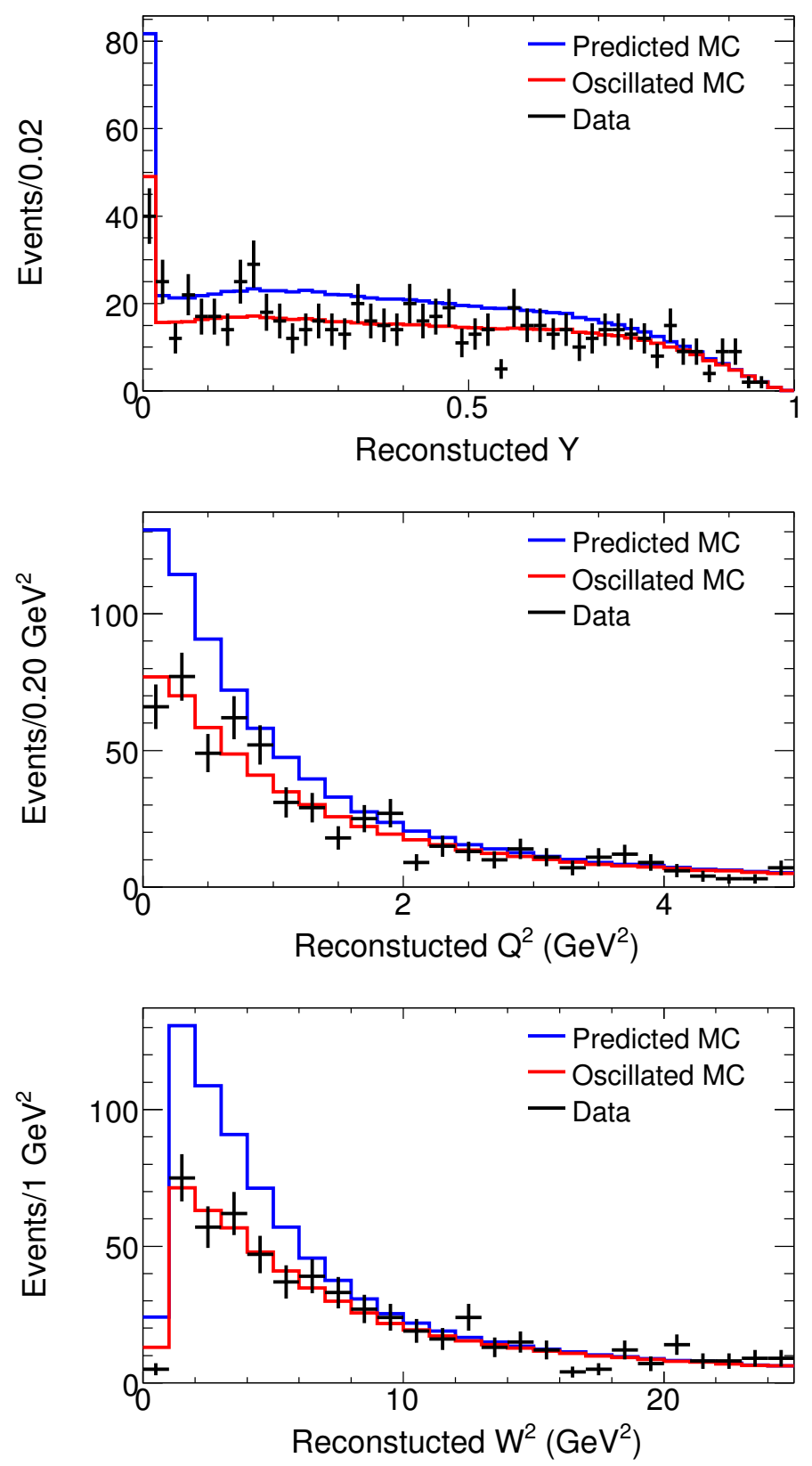

Figure 8.10: These figures show the selected $\nu_{\mu}$ charged-current far detector events. The top figure shows the reconstructed inelasticity parameter, Y. The middle figure shows the reconstructed momentum transfer, $Q^{2}$. The bottom figure shows the reconstructed invariant mass squared, $W^{2}$. These variables are defined in Section 3.3. 


\subsection{Summary}

In this chapter, the event selection procedure for the far detector events was described. The selection requirements are similar to the requirements for the near detector events, with three exceptions. First, the far detector events are required to be in coincidence with the neutrino arrival time, measured using the GPS clocks. Second, the far detector has a larger fiducial volume. Third, an additional requirement was introduced to minimize the number of background events due to cosmic ray muons. The selected far detector data events are accurately described using the oscillation hypothesis. The oscillation parameters will be measured in Chapter 9 . 


\section{Chapter 9}

\section{Measurement of muon neutrino disappearance}

As of July 2007, MINOS accumulated $1.27 \times 10^{20}$ protons on target in the Run I period and $1.94 \times 10^{20}$ protons on target in the Run II period. This exposure includes only the data from the low energy beam configuration. The 702 candidate $\nu_{\mu}$ charged-current events with energies between $0-120 \mathrm{GeV}$ are selected from the recorded far detector data. Assuming there are no neutrino oscillations, the expected number of $\nu_{\mu}$ charged-current events is 910 .

This chapter describes the oscillation analysis of the far detector events. First, the reconstructed energy spectra for the data events are compared to the MC expectation. Then, the oscillation parameters are measured by fitting the data events. Next, the method used to estimate the systematic errors for the measurement of the oscillation parameters is described. Finally, our results are compared with the current MINOS results.

\subsection{Description of the fit method}

The data events are analyzed using a simple two-neutrino oscillation hypothesis. It is assumed that the deficit of the observed events is only due to the $\nu_{\mu} \rightarrow \nu_{\tau}$ oscillations. Following the standard analysis of oscillations for 
the two massive neutrinos [5], the probability that $\nu_{\mu}$ reaches the far detector is given by the expression:

$$
P\left(\nu_{\mu} \rightarrow \nu_{\mu}\right)=1-\sin ^{2} 2 \theta \sin ^{2}\left(1.27 \Delta m^{2} \frac{L}{E}\right)
$$

where $\mathrm{L}[\mathrm{km}]$ is the distance from the NuMI target to the far detector, $\mathrm{E}[\mathrm{GeV}]$ is the neutrino energy, $\Delta m^{2}\left[\mathrm{eV}^{2}\right]$ is the difference of squared masses, and $\theta$ is the mixing angle.

The MC simulation predicts less than $1.5 \nu_{\tau}$ charged-current events from the $\nu_{\mu} \rightarrow \nu_{\tau}$ oscillations. Many of the oscillated $\nu_{\tau}$ are below the production threshold for $\nu_{\tau}$ charged-current interactions. The number of these interactions is significantly less than the event normalization uncertainty. The maximum number of expected $\nu_{\mathrm{e}}$ charged-current interactions is negligibly small. These $\nu_{\mathrm{e}}$ events can appear through the subdominant $\nu_{\mu} \rightarrow \nu_{\mathrm{e}}$ oscillations. The procedure to select $\nu_{\mu}$ charged-current events removes many $\nu_{\tau}$ charged-current and $\nu_{\mathrm{e}}$ charged-current events because these interactions look similar to background neutral-current events. The number of expected background neutral-current events is 4.1, assuming there are no neutrino oscillations.

The oscillation parameters are obtained by minimizing the following function $[5]$ :

$$
\chi^{2}\left(\left|\Delta m^{2}\right|, \sin ^{2} 2 \theta\right)=\sum_{i=1}^{N_{b i n}} 2\left(N_{i}^{e x p}-N_{i}^{o b s}\right)+2 N_{i}^{o b s} \ln \left(\frac{N_{i}^{o b s}}{N_{i}^{e x p}}\right)
$$

where $N_{b i n}$ is the number of histogram bins, $N_{i}^{\text {obs }}$ is the number of observed data events in the bin $i$, and $N_{i}^{e x p}$ is the number of expected MC events in 
the bin $i$. The value of $N_{i}^{e x p}$ depends on the oscillation parameters through weights of events contained in the bin $i$. These weights are adjusted using Equation 9.1.

The minimum of the function defined by Equation 9.2 (called a $\chi^{2}$ function) follows a $\chi^{2}$ distribution in the large sample limit. The $1 \mathrm{~d}$ Confidence Limits (C.L.) for the oscillation parameters $\left|\Delta m^{2}\right|$ and $\sin ^{2} 2 \theta$ are determined from the $\Delta \chi^{2}$ difference using the Gaussian approximation [5]: for 68\% C.L. $\Delta \chi^{2}=1$, for $90 \%$ C.L. $\Delta \chi^{2}=2.71$, and for $99 \%$ C.L. $\Delta \chi^{2}=6.63$.

The computation of the $\chi^{2}$ function defined in Equation 9.2 depends on histogram binning for the reconstructed neutrino energy. Two types of histograms are used for the analysis:

Histogram I Histogram contains 33 bins: 20 bins with $0.5 \mathrm{GeV}$ width from $0 \mathrm{GeV}$ to $10 \mathrm{GeV}$, 10 bins with $1 \mathrm{GeV}$ width from $10 \mathrm{GeV}$ to $20 \mathrm{GeV}$, 20-30 GeV bin, 30-50 GeV bin, and 50-200 GeV bin.

Histogram II Histogram contains 17 bins: 14 bins with $0.5 \mathrm{GeV}$ width from $0 \mathrm{GeV}$ to $7 \mathrm{GeV}, 7-8 \mathrm{GeV}$ bin, 8-10 GeV bin, and 10-200 GeV bin.

The primary fit procedure uses the four histograms shown in Figures 9.1 and 9.2. For each run period, two histograms are created. Histogram I is filled with the selected DIS events, and Histogram II is filled with the selected RES and QES events. The $\chi^{2}$ function is computed for the four pairs of histograms. The MC histograms are scaled to match the number of protons on target in the data. The MINUIT [108] program is used to minimize the $\chi^{2}$ function. 
The additional (secondary) fit procedure uses the two histograms shown in Figure 9.5. This fit does not separate the DIS events from the QES and RES events. The decision to designate a primary fit was based on the novelty of our approach to the analysis of the MINOS far detector events.

\subsection{Measurement of neutrino oscillations parameters}

The two oscillation parameters, $\left|\Delta m^{2}\right|$ and $\sin ^{2} 2 \theta$, are derived from a fit to the selected $\nu_{\mu}$ charged-current events. The fit minimizes the $\chi^{2}$ function defined in Equation 9.2. Table 9.1 summarizes the best fit parameters for the 6 oscillation fits; these 6 fits are described in the caption of Table 9.1. Figures 9.1, 9.2, and 9.3 shows the selected $\nu_{\mu}$ charged-current far detector events. These figures show the event rate as a function of the reconstructed event energy for the data events, the expected MC events without oscillations, and the MC events corrected with the best fit oscillation parameters.

Figures 9.4 and 9.6 show the ratios of the data spectra and the oscillated MC spectra to the MC spectra without oscillations as a function of the reconstructed neutrino energy. There is a clearly visible depletion in the number of data events. The shapes of the data distributions are accurately described by the two-neutrino oscillation hypothesis.

The additional (secondary) fits are also shown in Table 9.1. In these fits, the event energy is reconstructed as the sum of hadronic shower energy and muon energy (see Section 3.4). The secondary fit results are consistent with the primary fit results. 

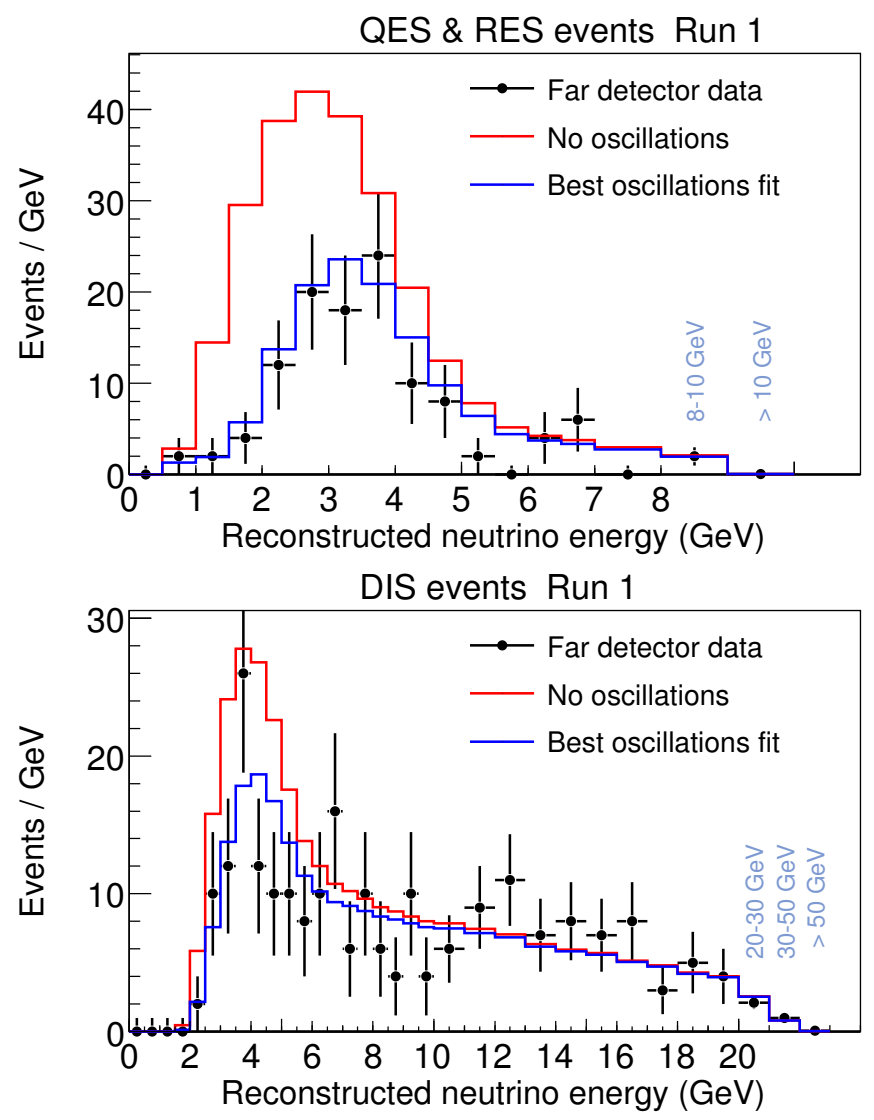

Figure 9.1: These figures show the two energy spectra used for the far detector oscillation fits. The top figure shows QES and RES events combined together. The bottom figure shows the DIS events. These figures show the Run I data events, the expected MC events without oscillations, and the oscillated MC events using the best fit oscillation parameters. 

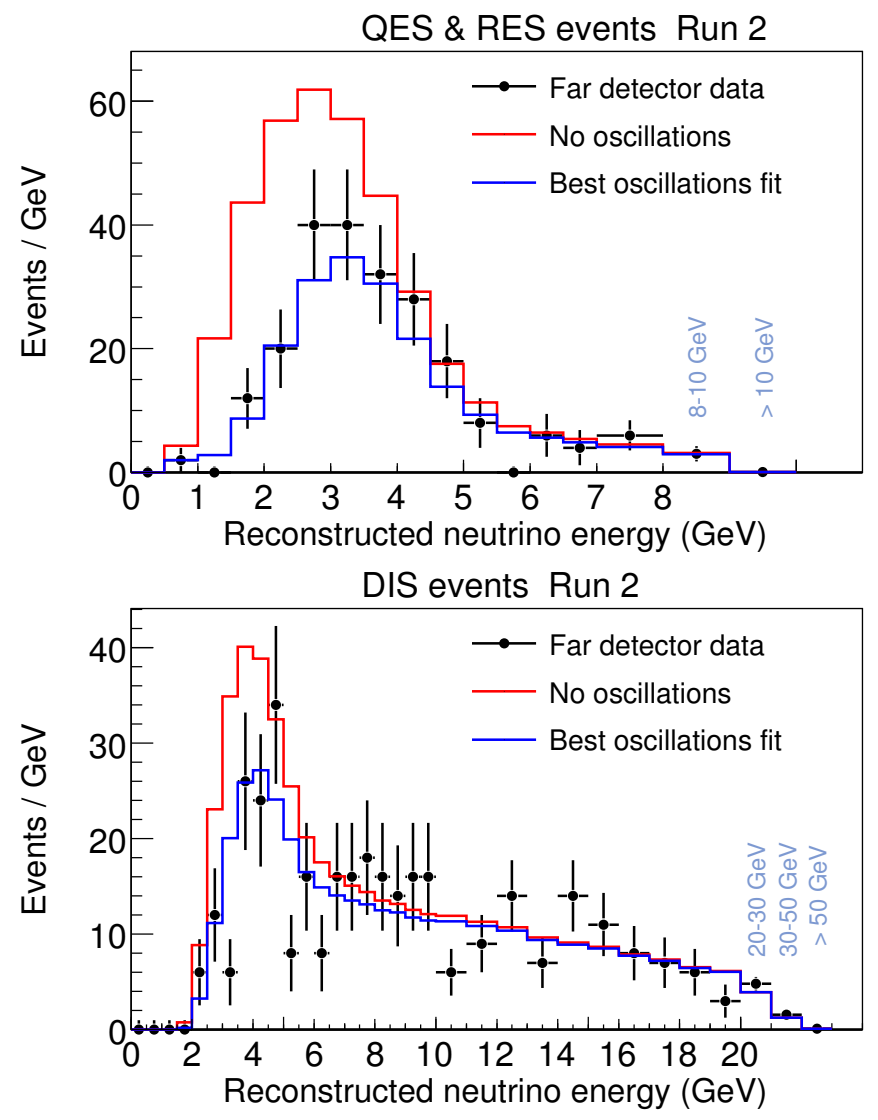

Figure 9.2: These figures show the two energy spectra used for the far detector oscillation fits. The top figure shows QES and RES events combined together. The bottom figure shows the DIS events. These figures show the Run II data events, the expected MC events without oscillations, and the oscillated MC events using the best fit oscillation parameters. 

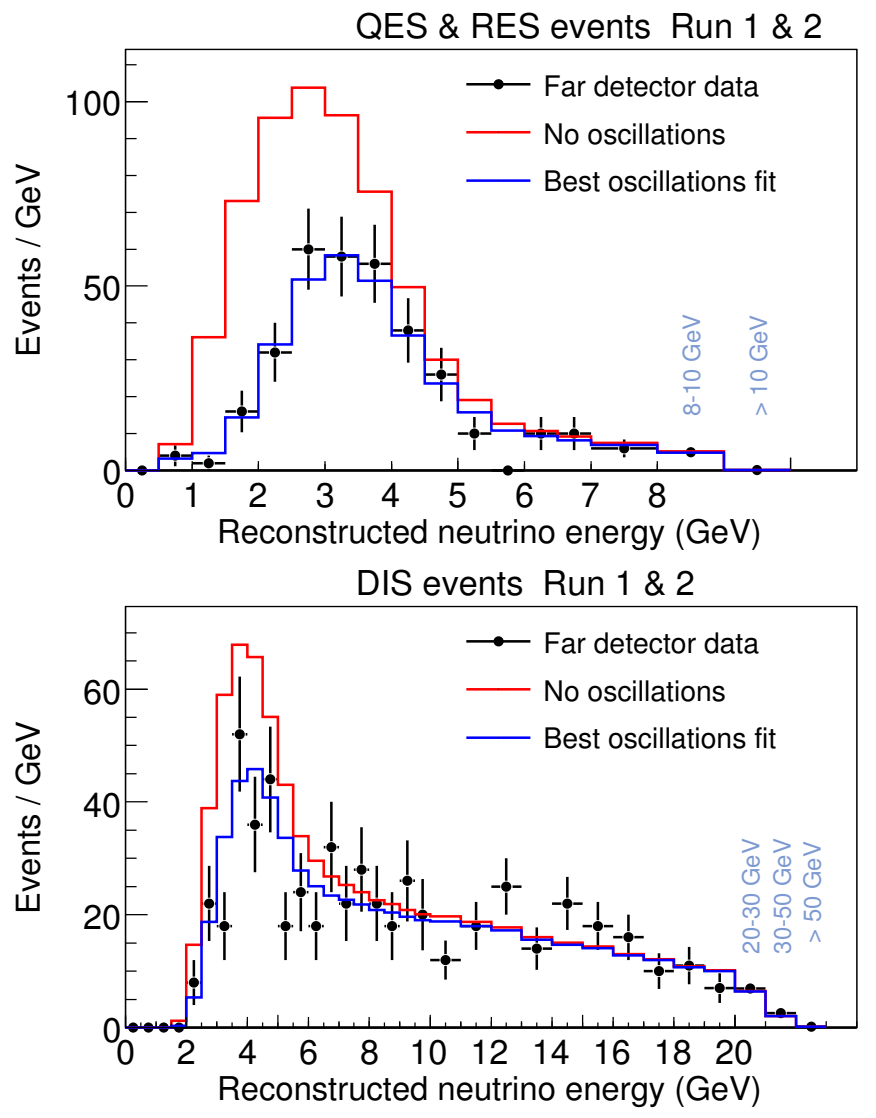

Figure 9.3: These figures show the two energy spectra. The top figure shows QES and RES events combined together. The bottom figure shows the DIS events. These figures show the sum of Run I and Run II data events, the expected MC events without oscillations, and the oscillated MC events using the best fit oscillation parameters. 

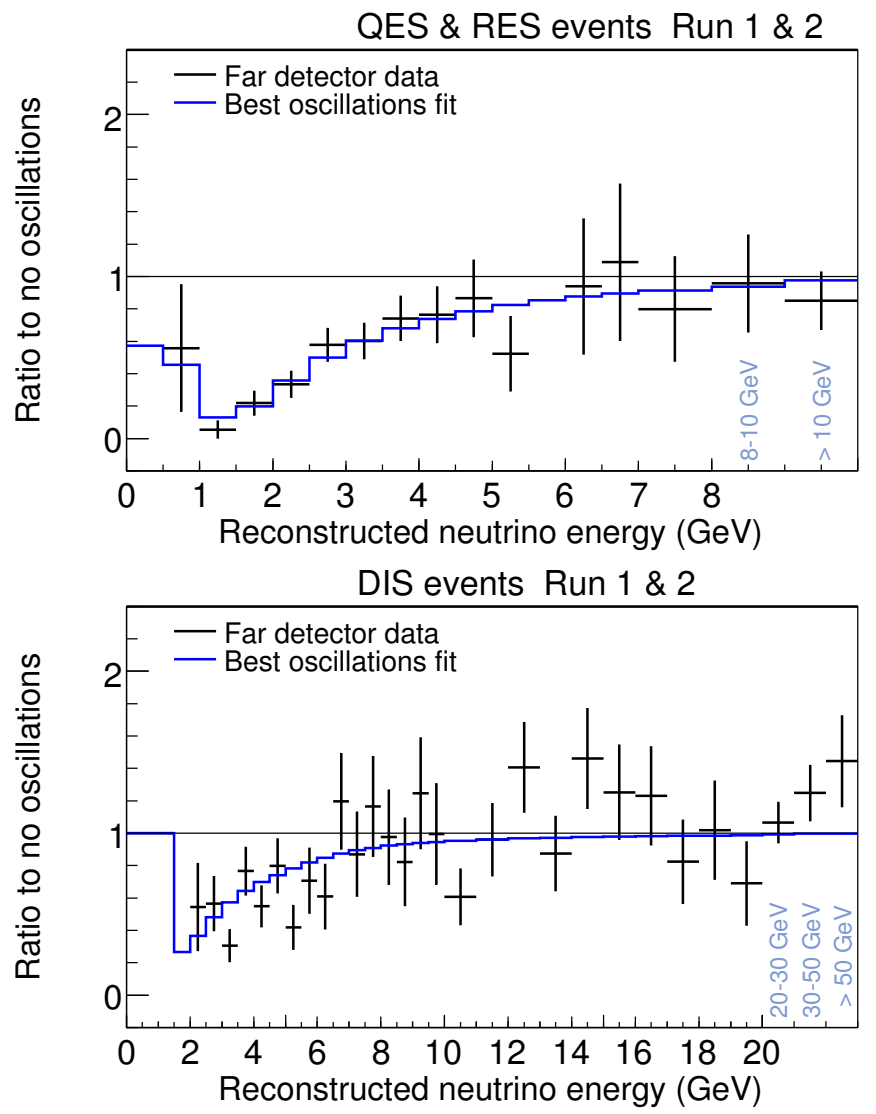

Figure 9.4: These figures show the data spectra and the oscillated MC spectra divided by the expected MC spectra without oscillations. The top figure shows QES and RES events. The bottom figure shows the DIS events. These figures show the sum of Run I and Run II data events. 

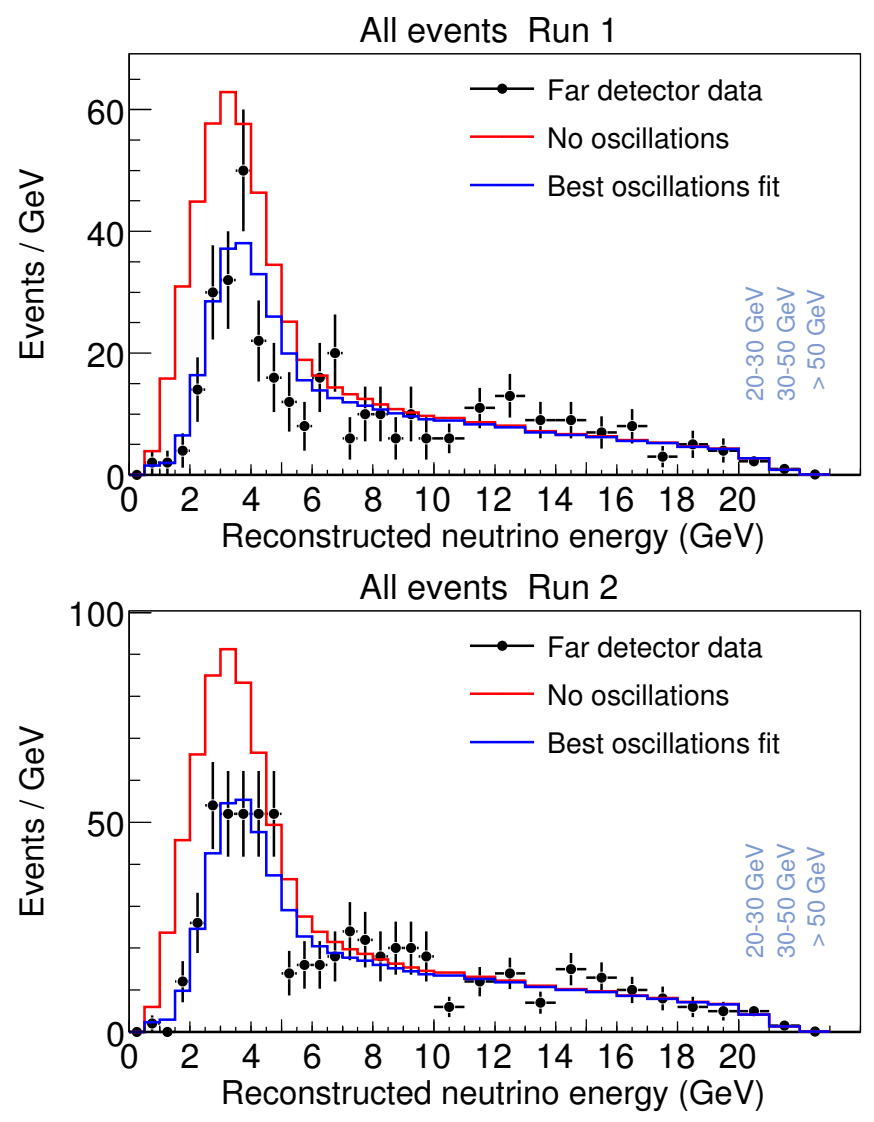

All events Run $1 \& 2$

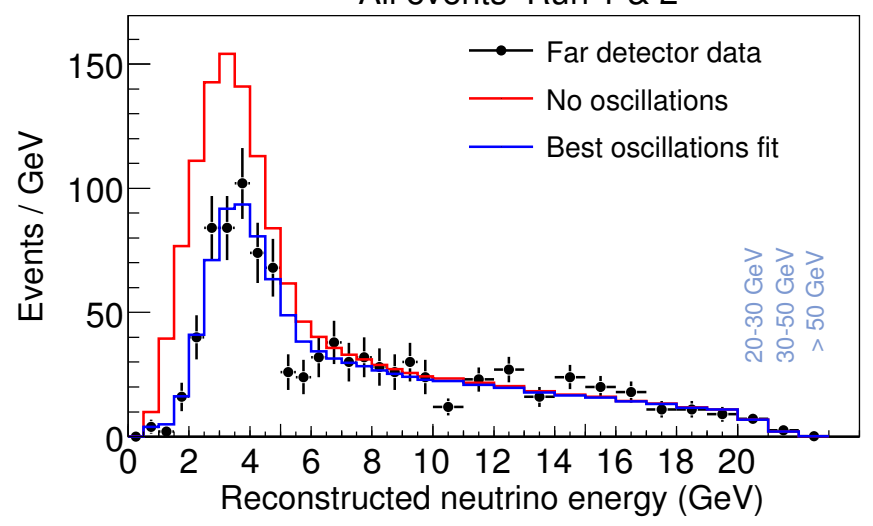

Figure 9.5: These figures show the data events, the expected $\mathrm{MC}$ events without oscillations, and the oscillated MC events using the best fit oscillation parameters. The top figure shows the Run I events. The middle figure shows the Run II events. These top two figures show the reconstructed energy spectra used for the oscillation fit. The bottom figure shows the sum of Run I and Run II events. 

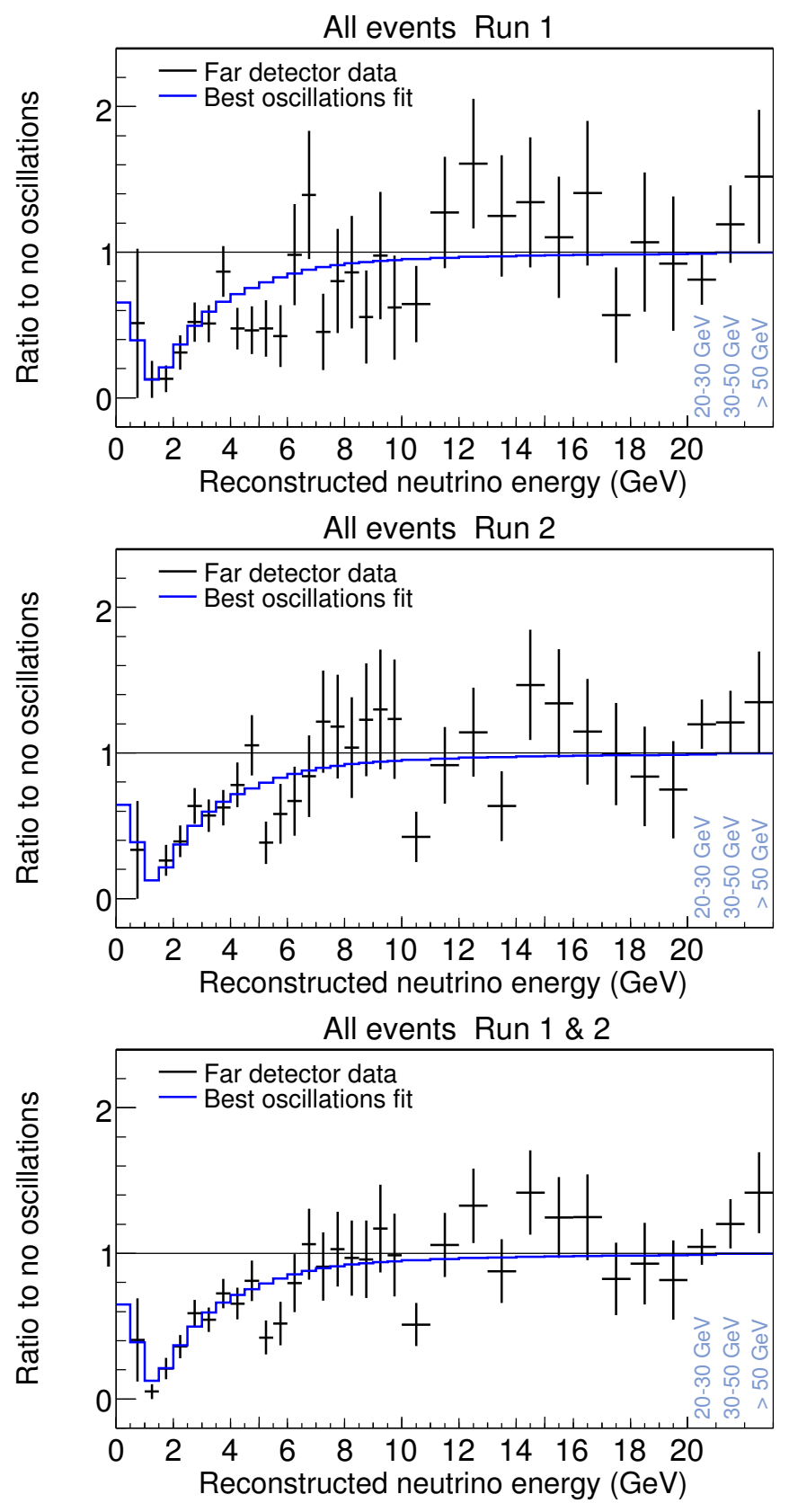

Figure 9.6: These figures show the data spectra and the oscillated MC spectra divided by the MC spectra without oscillations. The top figure shows Run I events. The middle figure shows Run II events. The bottom figure shows the sum of Run I and Run II events. 


\begin{tabular}{l|rc|rc|r|c} 
& $\sin ^{2} 2 \theta$ & Stat. & $\left|\Delta m^{2}\right|$ & Stat. & $\chi_{\text {init }}^{2}$ & $\chi_{\text {best }}^{2}$ \\
\hline \hline Primary Run I & 1.000 & -0.077 & 2.63 & \pm 0.18 & 109.1 & 36.7 \\
\hline Primary Run II & 1.000 & -0.047 & 2.33 & \pm 0.15 & 130.8 & 48.3 \\
\hline Primary all & 1.000 & -0.038 & 2.44 & \pm 0.12 & 240.0 & 86.5 \\
\hline \hline 2nd Run I & 1.000 & -0.071 & 2.64 & \pm 0.19 & 100.9 & 27.2 \\
\hline 2nd Run II & 1.000 & -0.044 & 2.34 & \pm 0.15 & 115.6 & 32.8 \\
\hline 2nd all & 1.000 & -0.034 & 2.45 & \pm 0.12 & 216.5 & 61.6
\end{tabular}

Table 9.1: Best fit oscillation parameters for 6 fit combinations. The primary method separates $\nu_{\mu}$ charged-current events into QES and RES events and DIS events. The secondary (2nd) method uses all $\nu_{\mu}$ charged-current events. The oscillation parameters are shown for the separate fits to the Run I events and the Run II events and the simultaneous fits for the Run I and Run II events. For $\left|\Delta m^{2}\right|$, statistical errors are determined as the largest deviation on the left and right sides from the best fit value for $68 \%$ C.L. interval. $\chi_{\text {init }}^{2}$ is the $\chi^{2}$ value for the data and MC prediction without oscillations. $\chi_{\text {best }}^{2}$ is the $\chi^{2}$ value for the data and $\mathrm{MC}$ weighted by best fit oscillations parameters.

The allowed 1d C.L. regions for the oscillation parameters using the primary fit method are:

$$
\begin{aligned}
& 68 \% \text { C.L.: } \sin ^{2} 2 \theta>0.96 \\
& 2.325 \times 10^{-3} \mathrm{eV}^{2}<\left|\Delta m^{2}\right|<2.554 \times 10^{-3} \mathrm{eV}^{2} \\
& 90 \% \text { C.L.: } \sin ^{2} 2 \theta>0.92 \\
& 2.249 \times 10^{-3} \mathrm{eV}^{2}<\left|\Delta m^{2}\right|<2.627 \times 10^{-3} \mathrm{eV}^{2} \\
& 99 \% \text { C.L.: } \sin ^{2} 2 \theta>0.87 \\
& 2.137 \times 10^{-3} \mathrm{eV}^{2}<\left|\Delta m^{2}\right|<2.732 \times 10^{-3} \mathrm{eV}^{2}
\end{aligned}
$$

For the primary fit method, the allowed regions at $68 \%, 90 \%$, and $99 \%$ C.L. for the $\left|\Delta m^{2}\right|$ and $\sin ^{2} 2 \theta$ oscillation parameters are shown in Figure 9.7. 


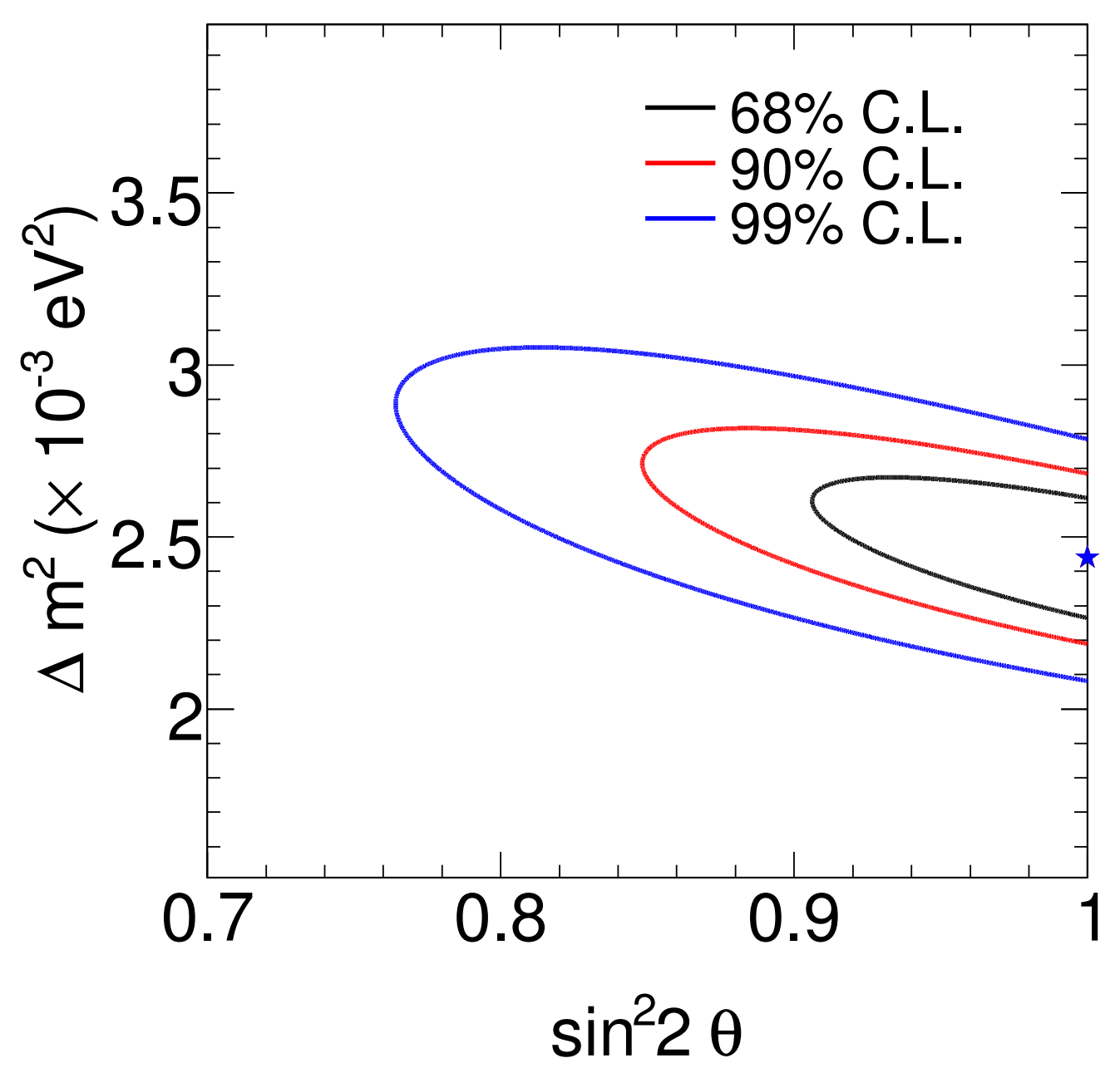

Figure 9.7: Allowed regions of $68 \%, 90 \%$, and $99 \%$ C.L in the $\left|\Delta m^{2}\right|$ and $\sin ^{2} 2 \theta$ plane for the primary fit of the far detector data. This fit separates $\nu_{\mu}$ charged-current events into the DIS events and the combination of QES and RES events. The best fit data point is shown as a blue star: $\left|\Delta m^{2}\right|=$ $2.44 \times 10^{-3} \mathrm{eV}^{2}$ and $\sin ^{2} 2 \theta=1$. 


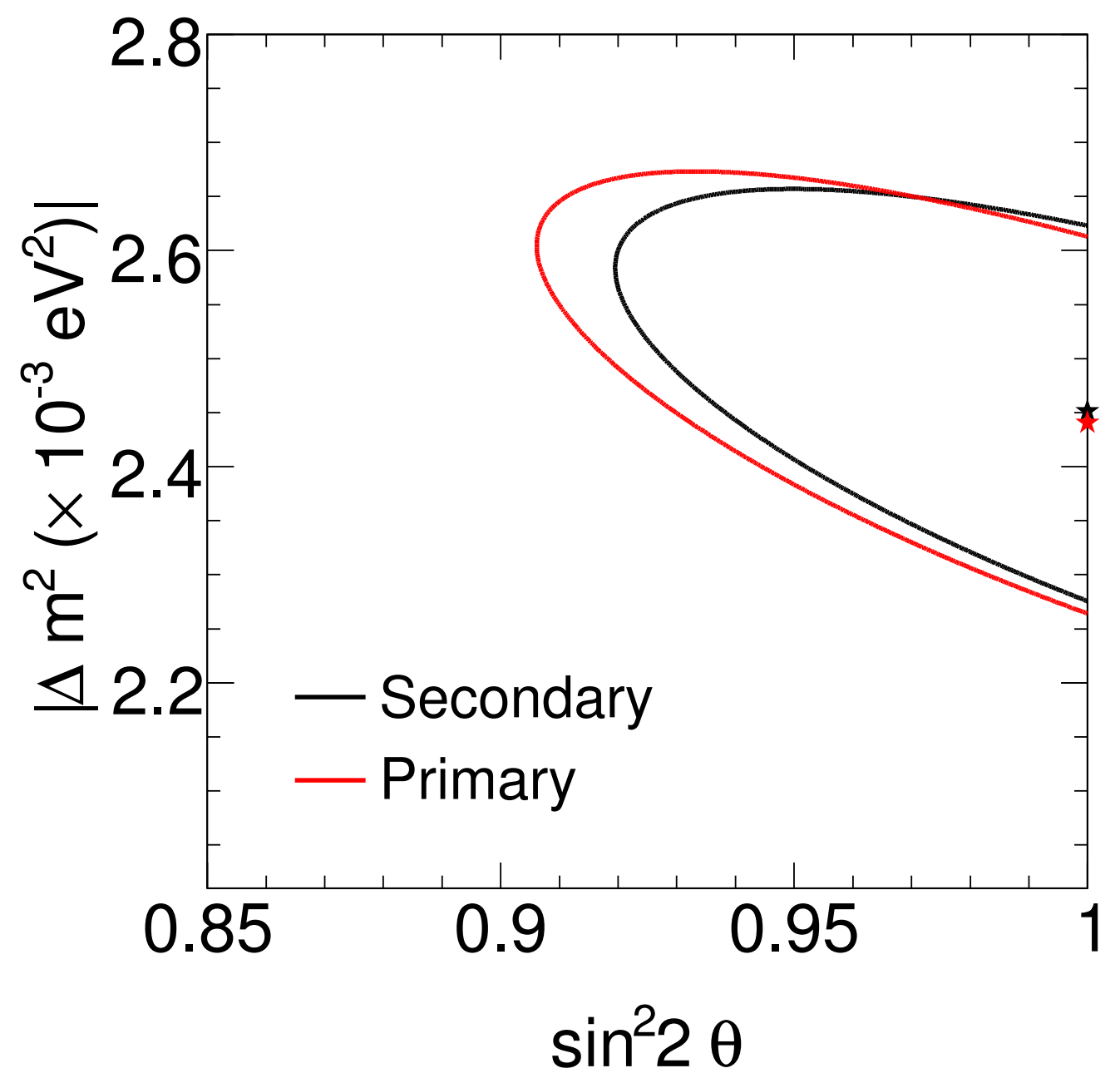

Figure 9.8: Allowed regions of $68 \%$ C.L in the $\left|\Delta m^{2}\right|$ and $\sin ^{2} 2 \theta$ plane for two fits of the far detector data. The region marked with a black line is obtained from the secondary fit. The region marked with a red line is obtained from the primary fit. Both fits are constrained to the physical region. 


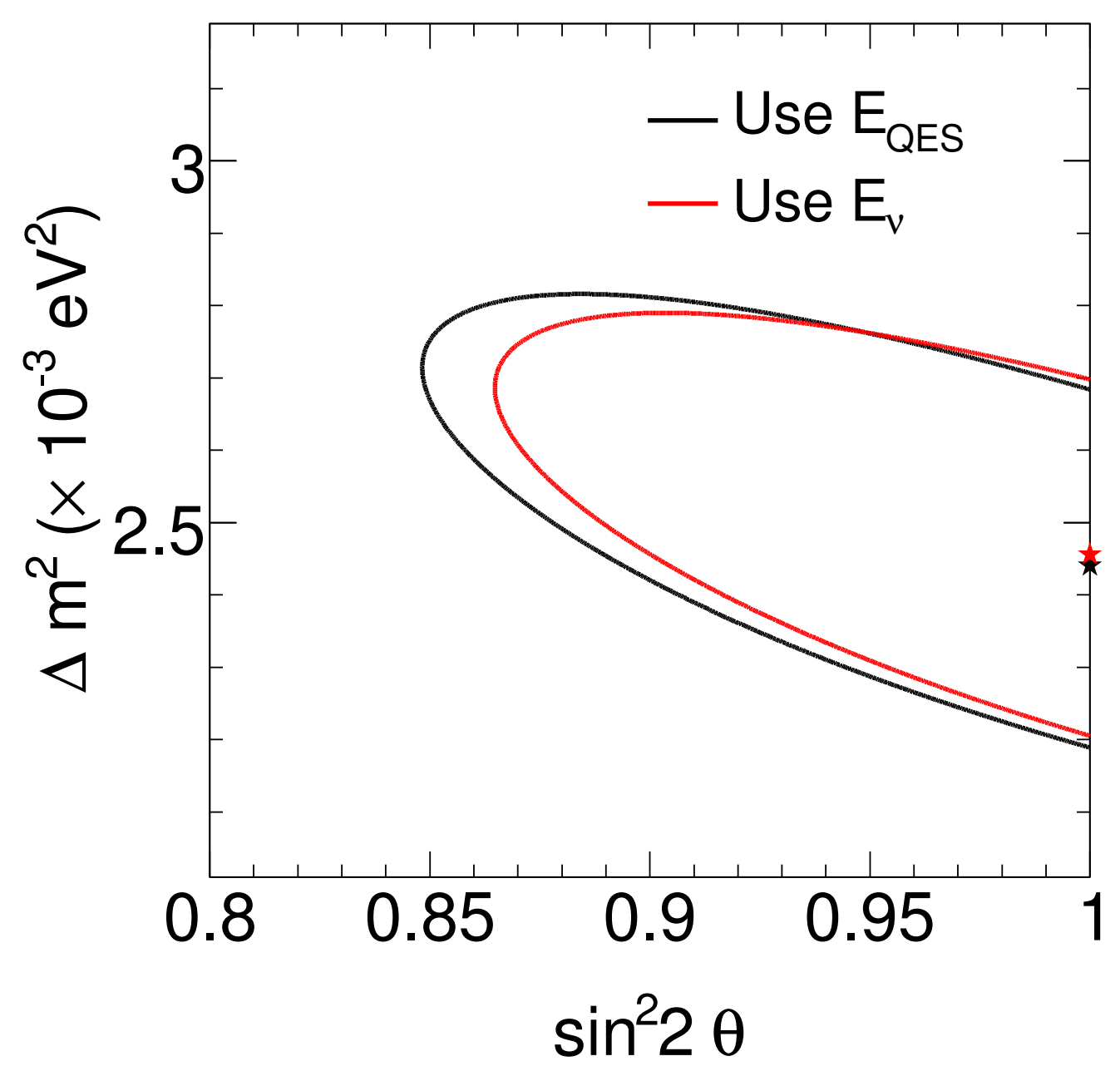

Figure 9.9: Allowed regions of $68 \%$ C.L in the $\left|\Delta m^{2}\right|$ and $\sin ^{2} 2 \theta$ plane for the primary fits of the far detector data. One fit uses the standard energy reconstruction formula and another fit uses the kinematic formula for the selected QES events. Both fits are constrained to the physical region. 


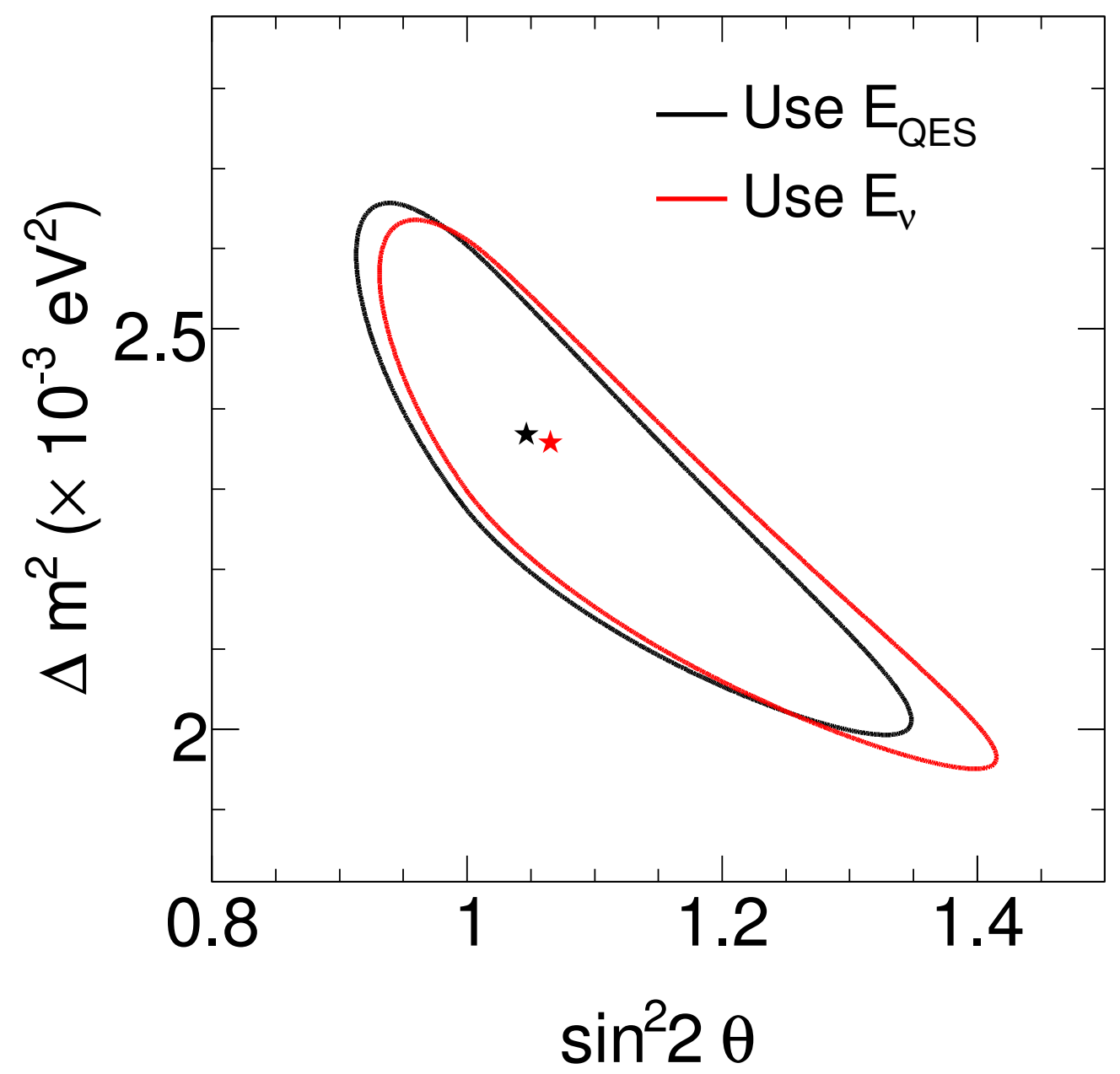

Figure 9.10: Allowed regions of $68 \%$ C.L in the $\left|\Delta m^{2}\right|$ and $\sin ^{2} 2 \theta$ plane for the primary fits of the far detector data. One fit uses the standard energy reconstruction formula and another uses the kinematic formula for the selected QES events. Both fits are not constrained to the physical region. 
The allowed regions are determined using the Gaussian approximation; the contour is defined as $\Delta \chi^{2}=2.3,4.6$, and 9.2 for $68 \%$ C.L., $90 \%$ C.L., and $99 \%$ C.L. respectively.

Figure 9.8 shows the allowed $68 \%$ C.L. regions for the primary and secondary fit methods. The secondary method has the smaller allowed C.L. region. This difference could be explained by statistical and systematic errors. Figures 9.9 and 9.10 show the allowed $68 \%$ C.L. regions for the primary fit methods using two energy reconstruction methods (see Section 3.4). One fit uses the kinematic formula defined in Equation 3.5, and another fit uses the simple formula defined in Equation 3.1. The kinematic formula reduces the energy reconstruction bias for the selected QES events. For this fit, the best fit point is closer to the physical region. This comparison of the two approaches suggests a presence of (small) systematic effect possiblly not modeled by the MC simulation. The two fits agree within the statistical errors. A study of the near detector events (see Section 7.6) does not show differences between the two energy reconstruction methods.

\subsection{Statistically independent pseudo-experiments}

The fit procedure is tested using MC pseudo-experiments. The far detector $\mathrm{MC}$ simulation contains $2.5545 \times 10^{23}$ protons on target (POT). The MC pseudo-experiments are created by dividing these MC events into statistically independent subsamples. Each subsample contains the same number of POT as the data, but the number of events is allowed to fluctuate following Poisson 
statistics. The pseudo-experiments are created following this procedure:

1. Apply the corrections derived in Chapter 7 to all the MC events.

2. Compute mean number of events: $N_{R u n}$ I per $1.27 \times 10^{20}$ POT for the Run I period and $N_{\text {Run II }}$ per $1.94 \times 10^{20}$ POT for the Run II period. All MC events are used to compute these means.

3. Pick a random number of events, $N_{R u n}^{\text {pseudo }}$, from the Poisson distribution with the $N_{\text {Run I }}$ mean. Pick a random number of events, $N_{\text {Run II }}^{\text {pseudo }}$, from the Poisson distribution with the $N_{\text {Run II }}$ mean.

4. Select $N_{R u n ~ I}^{\text {pseudo }}$ and $N_{R u n ~ I I}^{\text {pseudo }}$ independent events from the MC simulation. These events make one pseudo experiment for the Run I and Run II data.

5. Apply oscillation weights to the selected events using Equation 9.1.

The 600 statistical pseudo-experiments are generated using the oscillation parameters obtained using the primary fit method and the data from both run periods. These oscillation parameters are listed in Table 9.1. The events from the pseudo-experiments are fitted in place of the data events. Figure 9.11 shows the sums of the (pseudo) data events from the Run I and Run II periods for the 600 pseudo-experiments. Figure 9.11 also shows the number of $\mathrm{MC}$ events at the best oscillation fit parameters. The results of the oscillation fits for 600 pseudo-experiments are shown in Figure 9.13. The widths 


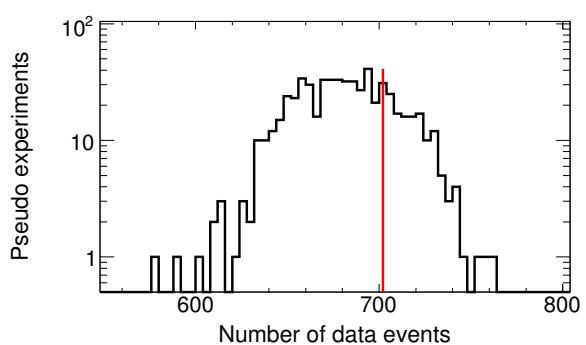

(a)

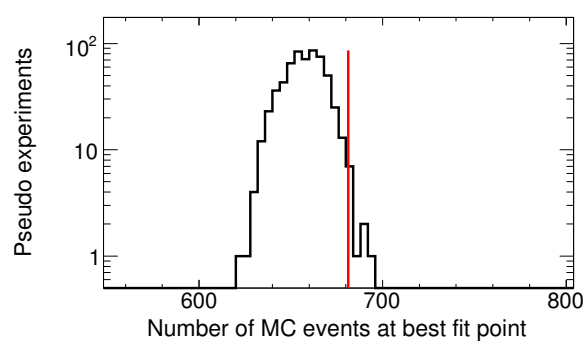

(b)

Figure 9.11: Results of 600 statistically independent pseudo-experiments. (a) number of data events in a pseudo experiment. (b) number of events in a best fit $\mathrm{MC}$ simulation, where fit parameters are constrained to the physical region: $\sin ^{2} 2 \theta \leq 1$. Values obtained from the fit to the far detector data are shown as red lines.

of distributions indicate variations in the fit quantities due to the statistical fluctuations.

The far detector data suggest the maximal value for the mixing angle with $\sin ^{2} 2 \theta=1$. In the above pseudo-experiments, the best fit point is frequently located near the physical boundary at $\sin ^{2} 2 \theta=1$, as shown in Figure 9.12. The effect of the statistical fluctuations is studied using the pseudo-experiments. The parameter $\sin ^{2} 2 \theta$ can be treated as a free parameter, and it is allowed to vary between 0 and 2. The results for these 600 pseudo-experiments are shown in Figure 9.14. In more than half of these pseudo-experiments, the best fit point moves into the unphysical region. This study suggests that the MINOS experiment does not have significant sensitivity to this parameter region. Based on this study, the constrained fit was used for the oscillation analysis. 


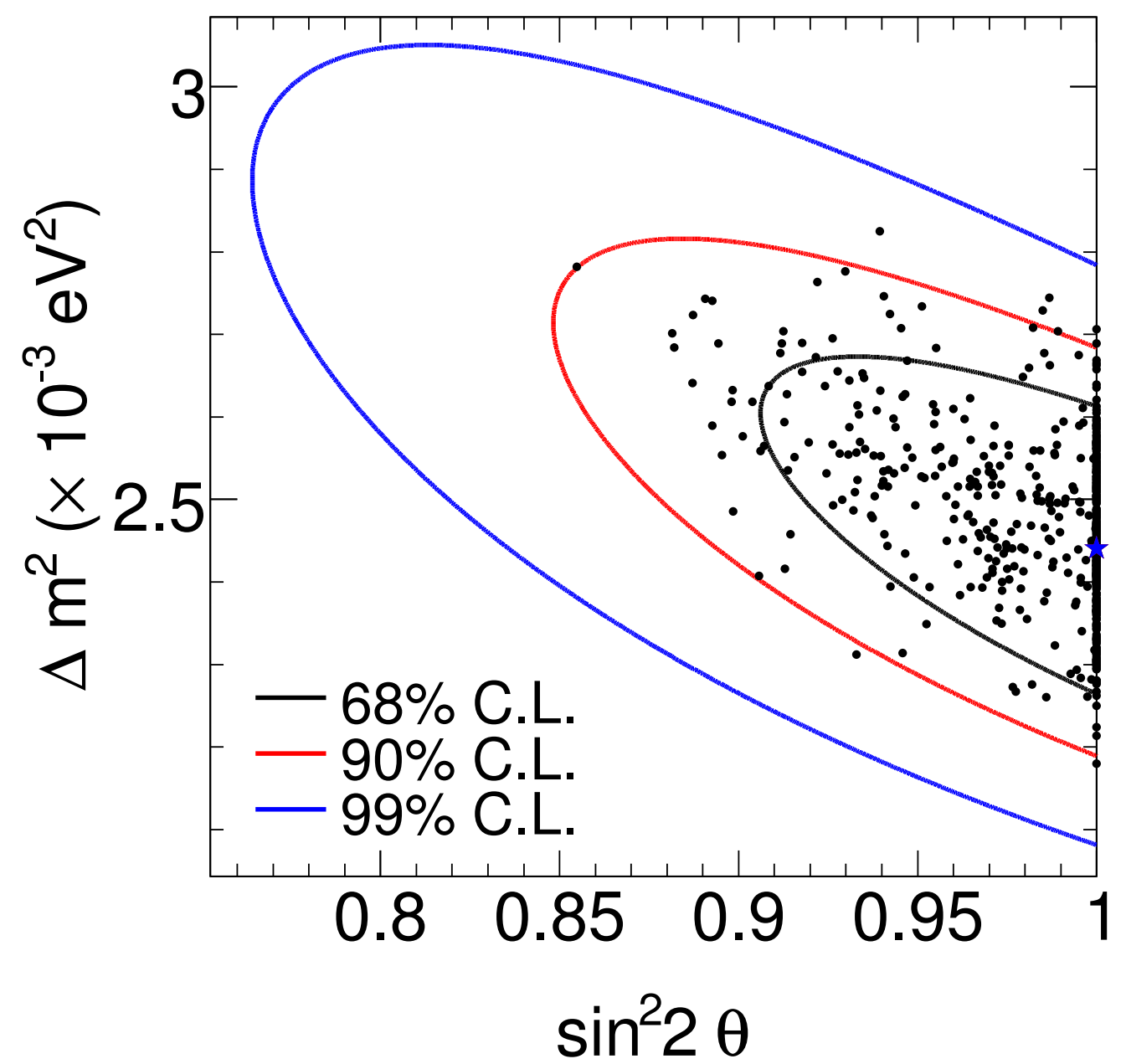

Figure 9.12: Allowed regions of $68 \%, 90 \%$, and 99\% C.L in the $\left|\Delta m^{2}\right|$ and $\sin ^{2} 2 \theta$ plane for the primary fit method. The best fit data point is shown as a blue star: $\left|\Delta m^{2}\right|=2.44 \times 10^{-3} \mathrm{eV}^{2}$ and $\sin ^{2} 2 \theta=1$. Best fit points for the 600 statistically independent pseudo-experiments are shown as black points. 


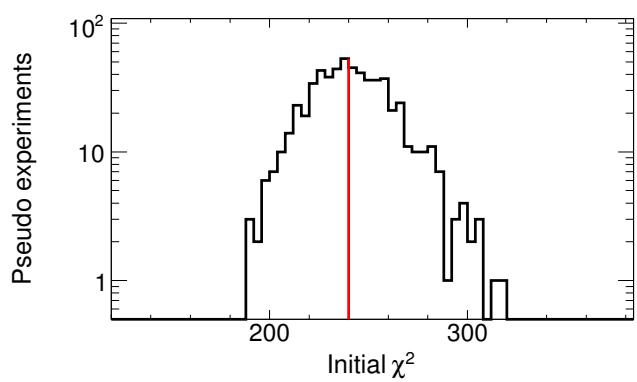

(a)

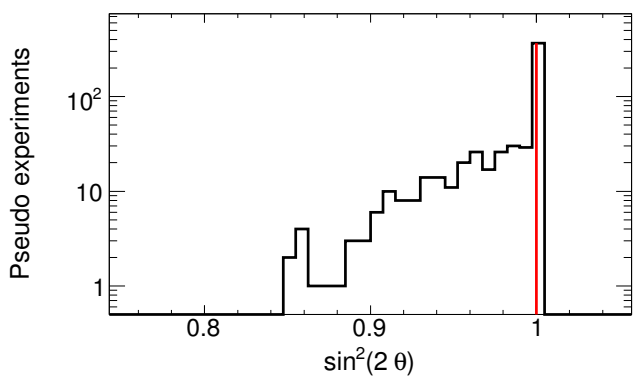

(c)

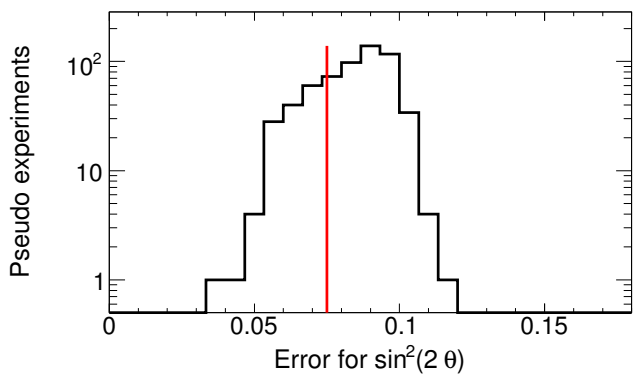

(e)

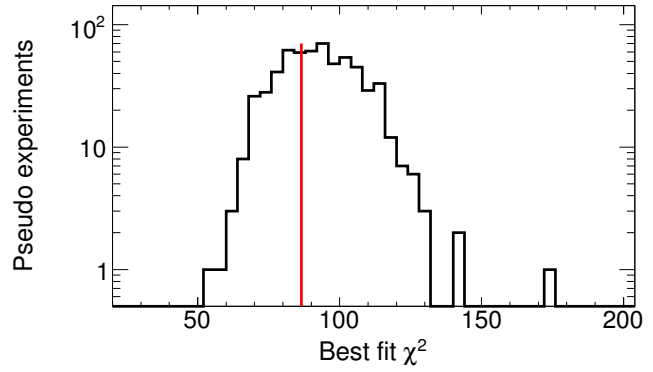

(b)

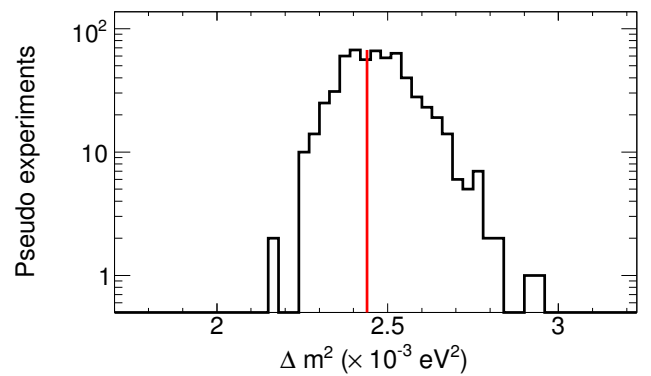

(d)

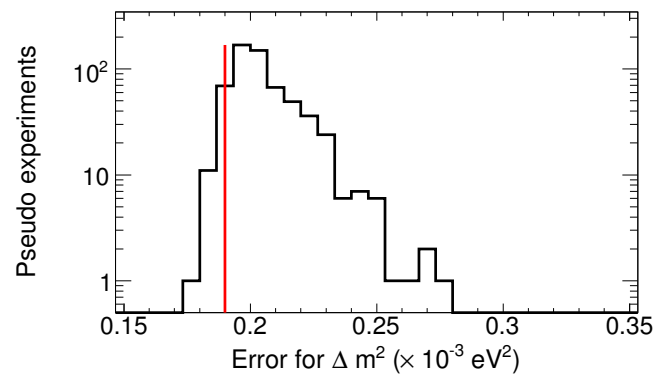

(f)

Figure 9.13: Results of 600 statistically independent pseudo-experiments. Fit parameters are constrained to the physical region: $\sin ^{2} 2 \theta \leq 1$. (a) the $\chi^{2}$ values for the data and MC without oscillations. (b) the $\chi^{2}$ values for the data and the best oscillation fit MC. (c) and (d) the best fit parameters. (e) and (f) $90 \%$ C.L. statistical errors for the best fit parameters. Values obtained from the fit to the real far detector data are shown as red lines; these values were used to generate the pseudo-experiments. 


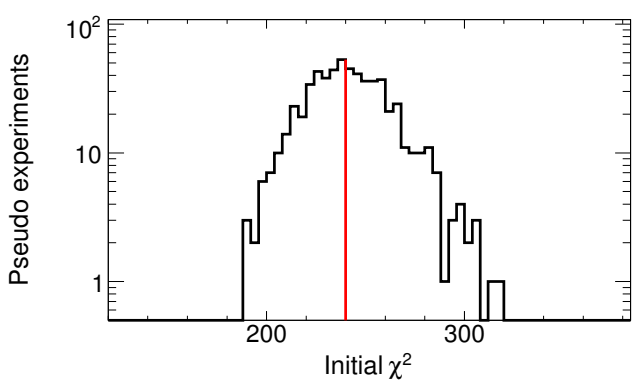

(a)

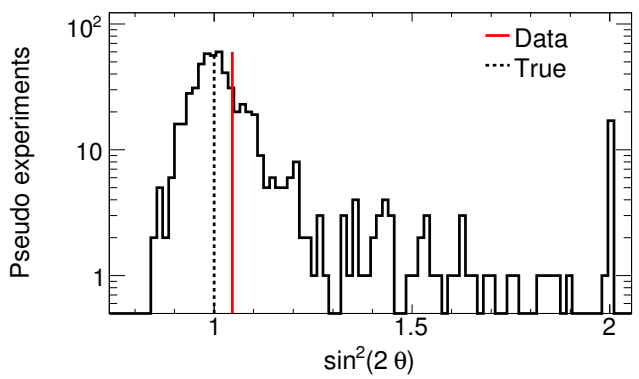

(c)

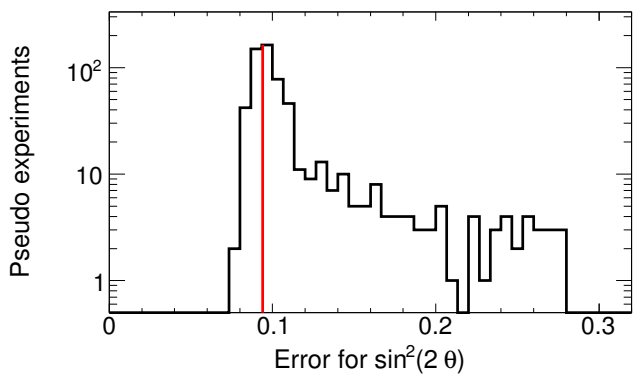

(e)

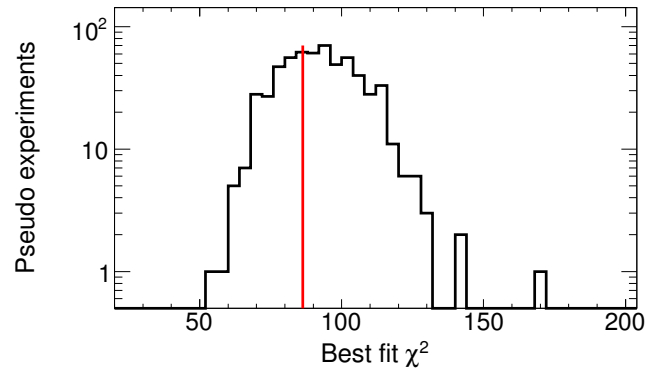

(b)

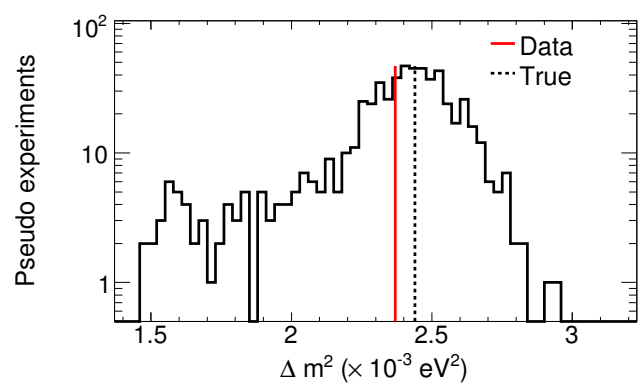

(d)

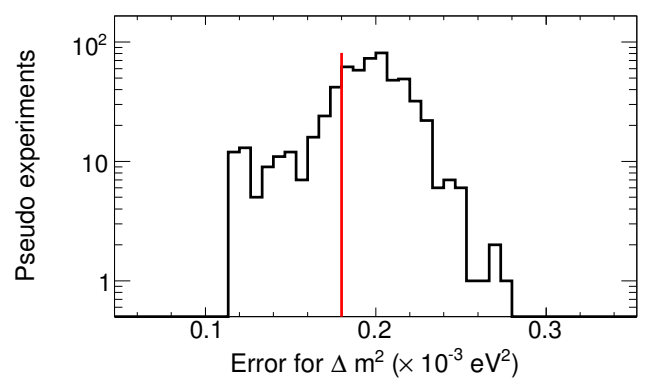

(f)

Figure 9.14: Results of 600 statistically independent pseudo-experiments. Fit parameters are not constrained to the physical region, but with the requirement $\sin ^{2} 2 \theta \leq 2$. (a) the $\chi^{2}$ values for the data and MC without oscillations. (b) the $\chi^{2}$ values for the data and best oscillation fit. (c) and (d) the best fit parameters. (e) and (f) $90 \%$ C.L. statistical errors for best fit parameters. Values obtained from the fit to the real far detector data are shown with red lines. Black dotted lines show the true values of oscillation parameters used to generate the pseudo-experiments. 


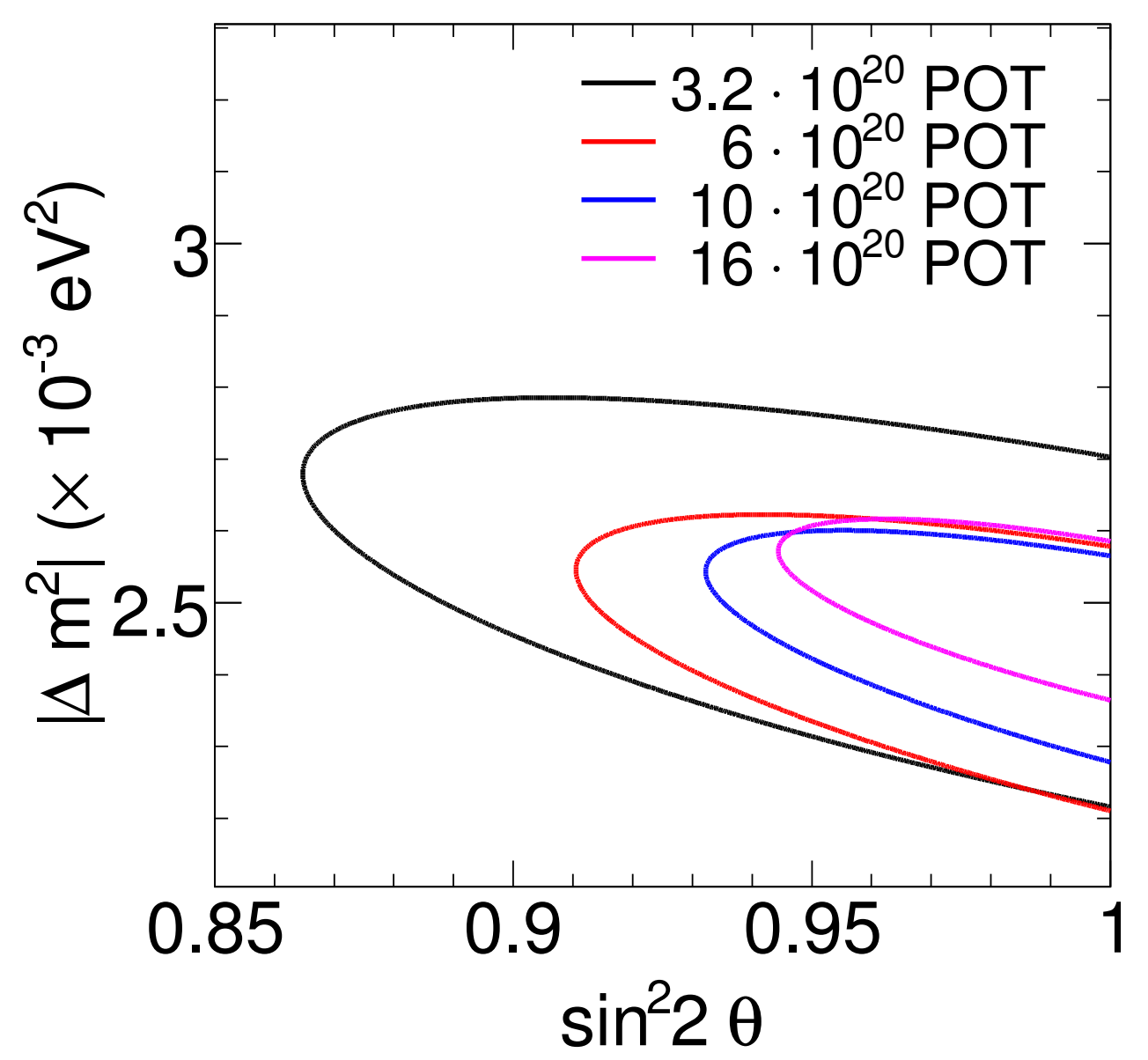

Figure 9.15: Allowed regions of $90 \%$ C.L. in the $\left|\Delta m^{2}\right|$ and $\sin ^{2} 2 \theta$ plane for four simulated pseudo-experiments with several values of the number of protons on target (POT). These contours are obtained using the primary fit method. 


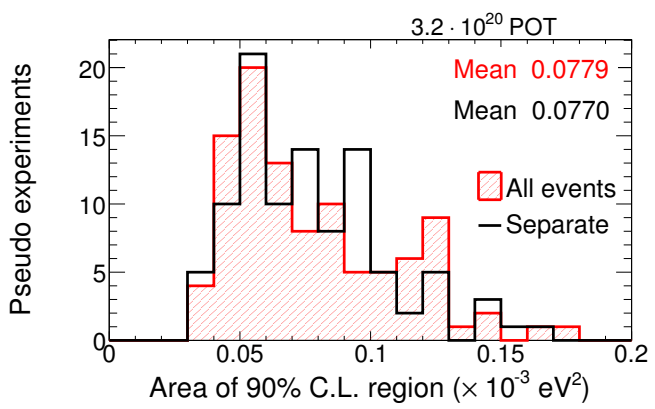

(a)

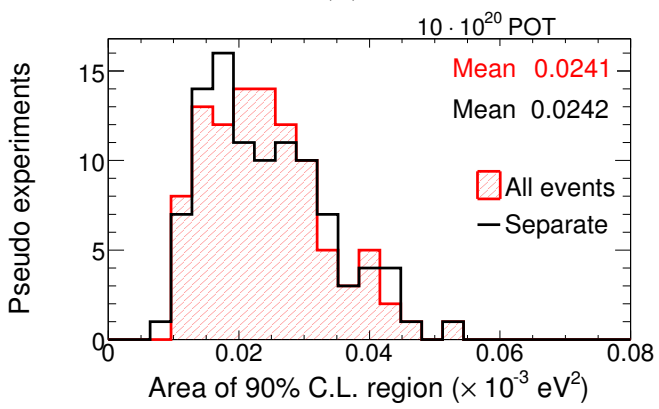

(c)

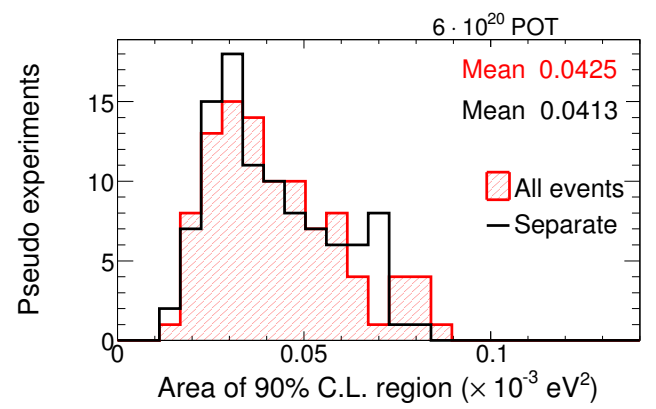

(b)

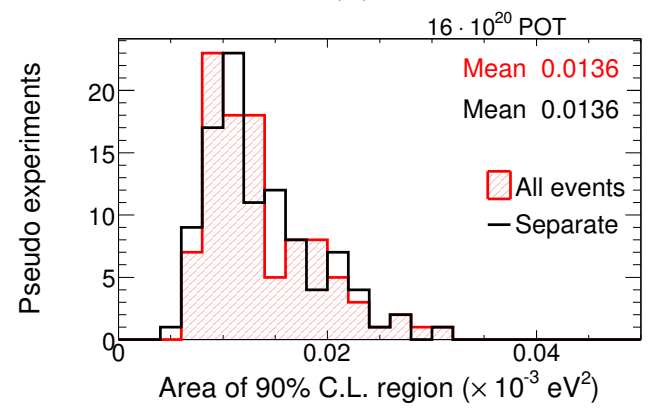

(d)

Figure 9.16: Illustrations of the statistical errors for the four sets of pseudoexperiments with several values of the number of protons on target (POT). The figures show the area contained within the 90\% C.L. (see Figure 9.15) for one hundred pseudo-experiments at each fixed number of POT. The two fit methods are compared in these figures. The primary fit method is shown with a black line, labeled as "Separate." The secondary fit method is shown with a red histogram, labeled as "All events." 
The primary and secondary fit methods are compared using several values of POT: $3.2 \times 10^{20}, 6 \times 10^{20}, 10 \times 10^{20}$, and $16 \times 10^{20}$ POT. MINOS is expected to record between $10 \times 10^{20}$ and $16 \times 10^{20}$ POT by the end of its operations. For each exposure, one hundred pseudo-experiments are generated using the above described procedure, where only a single run period is used. These pseudo experiments are fitted using the primary and secondary fit methods. For example, the allowed regions of $90 \%$ C.L. are shown in Figure 9.15 for four individual pseudo-experiments. For each of the pseudo-experiments, the area of the allowed region of $90 \%$ C.L. is computed. These areas are shown in Figure 9.16. There are no statistically signficant differences between the primary and secondary fit methods. Thus, the two methods have similar statistical sensitivity for the $\left|\Delta m^{2}\right|$ and $\sin ^{2} 2 \theta$ parameters using the (expected) final MINOS data set.

\subsection{Treatment of the systematic errors}

The systematic uncertainties can be broadly separated into two categories. First, there are uncertainties associated with the extrapolation from the near detector to the far detector (extrapolation uncertainties). Second, there are uncertainties associated with the measurement of the far detector events properties (far detector uncertainties). In this section, the systematic errors for the primary fit method are computed. The primary fit method is described in Section 9.1.

The extrapolation uncertainties are studied by varying the MC tuning 
parameters derived in Chapter 7. The MC prediction is generated using the parameters obtained in the fit to the near detector data. The 9 additional sets of parameters are obtained in order to study the dependence of the fit parameters on the selection procedure. These additional sets are described in Sections 6.2 and 7.5. The systematic uncertainties for the measurement of the oscillation parameters are studied by comparing the default extrapolation with these 9 additional extrapolations.

The 10 sets of parameters (default plus 9 additional sets) are applied to the 600 statistically independent pseudo-experiments described in Section 9.3. This procedure generates 6000 pseudo-experiments. Each pseudo experiment is fitted in place of the real data events. Figure 9.17 shows the results of the oscillation fits to 6000 pseudo-experiments. The results from these pseudoexperiments are consistent with the results obtained from the fits to the real far detector data.

There are 6 major far detector systematic uncertainties for the measurement of the oscillation parameters [46]. The far detector systematic uncertainties are studied by varying the following 6 parameters in the far detector MC simulation:

(a) $\pm 10.3 \%$ uncertainty on the absolute hadronic shower energy,

(b) $\pm 3.3 \%$ uncertainty on the relative hadronic shower energy between the near and far detectors, 
(c) $\pm 4.0 \%$ uncertainty on the far detector rate, which is the sum in quadratures of the uncertainties on the detectors's fiducial mass, event selection efficiency, and the counting number of protons on target.

(d) $\pm 50 \%$ uncertainty on the neutral-current contamination among the selected $\nu_{\mu}$ charged-current events,

(e) $\pm 2.0 \%$ uncertainty on the muon momentum measured via the range, (f) $\pm 3.0 \%$ uncertainty on the muon momentum measured via the curvature.

The \pm shifts for each of the 6 uncertainties are applied to the 100 statistically independent pseudo-experiments. This procedure generates 1200 pseudo-experiments. These 1200 pseudo-experiments are compared to the 100 pseudo-experiments without the systematic uncertainties. The results of fitting the combined 1300 pseudo-experiments are shown in Figure 9.18.

The above approach using single parameter \pm shifts does not take into account correlations among the systematic effects. These correlations are studied using an additional set of pseudo-experiments. This set is generated by requiring simultaneous \pm shifts for the 6 systematic uncertainties, described above. For example, $(-,-,-,-,-,-)$ generates one set of the systematic uncertainties. The next set is generated by flipping $\mathrm{a}-$ for $\mathrm{a}+$ for one of the parameters. There are a total of $2^{6}=64$ combinations of these systematic shifts. Each combination is applied to the 100 statistically independent pseudo-experiments. These 6400 pseudo-experiments are compared to the 100 


\begin{tabular}{l|r|r} 
& $\sin ^{2} 2 \theta$ syst. error & $\left|\Delta m^{2}\right|$ syst. error $\left(\times 10^{-3} \mathrm{eV}^{2}\right)$ \\
\hline Extrapolation & 0.00995 & 0.0749 \\
\hline Single shift & 0.00604 & 0.0514 \\
\hline Multiple shifts & 0.01839 & 0.1312 \\
\hline Total & 0.02176 & 0.1596
\end{tabular}

Table 9.2: Systematic errors for the oscillation parameters. The extrapolation error is obtained using 10 sets of the parameters obtained using the near detector data. The single shift error is obtained using the single \pm shift for one of 6 systematic uncertainties. The multiple shifts error is obtained using the multiple \pm shifts for 6 systematic uncertainties. The total error is the sum in quadratures of the three errors.

pseudo-experiments without the systematic effects. The results of fitting the 6500 pseudo-experiments are shown in Figure 9.19.

The systematic error of measuring the oscillation parameters is computed using differences between two best fit parameters. First parameter is obtained from the fit to the pseudo-experiment with the systematic effects applied; second parameter is obtained from the fit to the same pseudo experiment, but without the systematic effects. These differences measure shifts in the oscillation parameters due to the systematic errors. The same systematic uncertainty can produce a different shift for two statistically independent pseudo-experiments. Figure 9.20 shows these differences for $\left|\Delta m^{2}\right|$ and $\sin ^{2} 2 \theta$ oscillation parameters. The largest differences are caused by simultaneous shifts in the 6 systematic uncertainties. The systematic errors of the oscillation parameters are determined by a symmetric interval around zero that contains at least $68 \%$ of the pseudo-experiments. Table 9.2 lists the values of the systematic errors for $\left|\Delta m^{2}\right|$ and $\sin ^{2} 2 \theta$. 


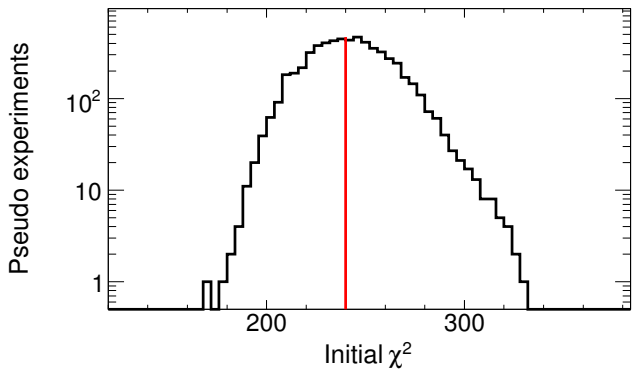

(a)

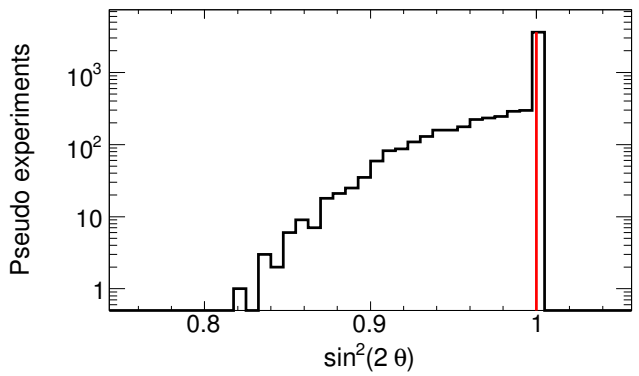

(c)

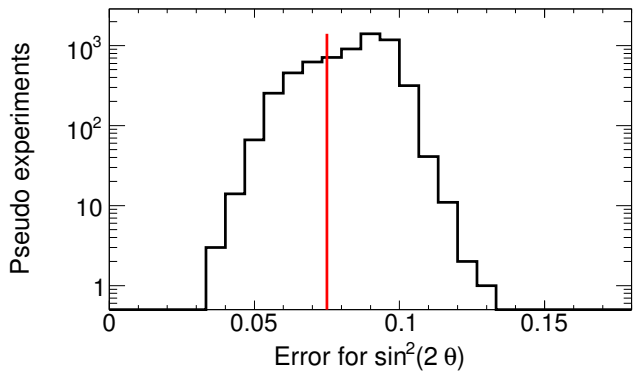

(e)

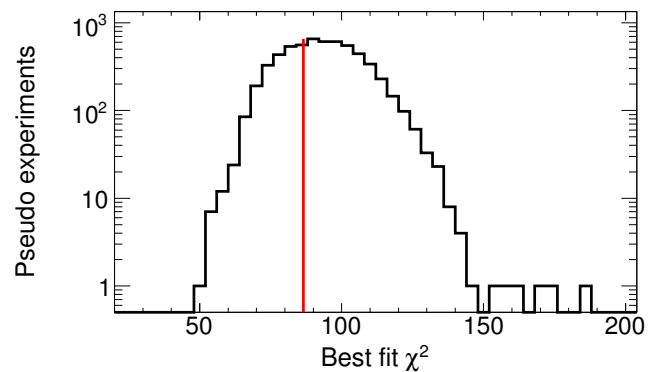

(b)

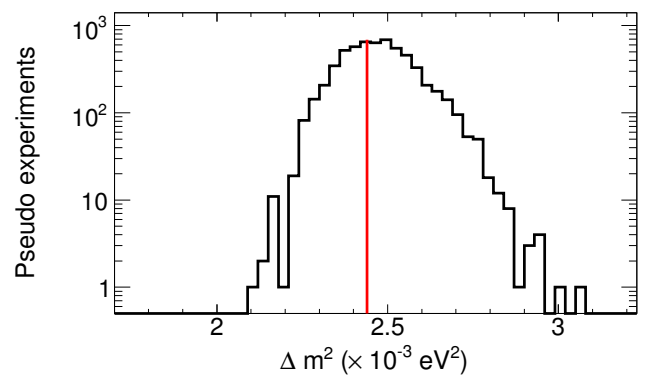

(d)

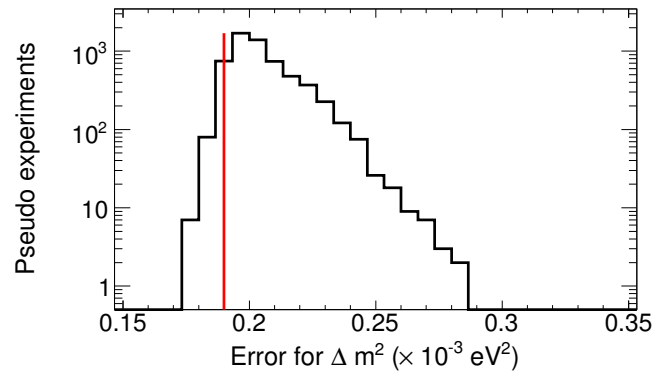

(f)

Figure 9.17: Results of 6600 independent pseudo-experiments for the extrapolation systematic errors, as discussed in the text. (a) the $\chi^{2}$ values for the data and MC without oscillations. (b) the $\chi^{2}$ values for the data and best oscillation fit MC. (c) and (d) the best fit oscillation parameters. (e) and (f) 90\% C.L. statistical errors for the best fit parameters. Red lines show the values obtained from the oscillation fit to the real far detector data. 


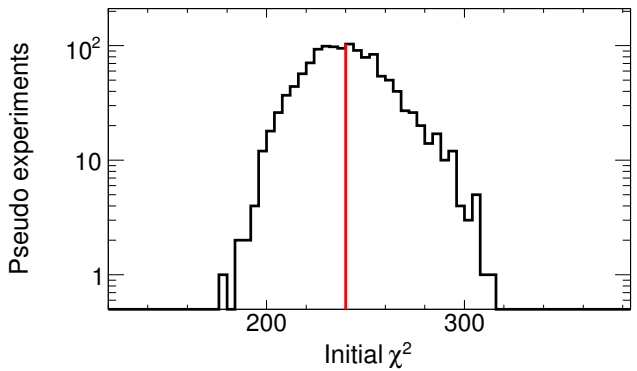

(a)

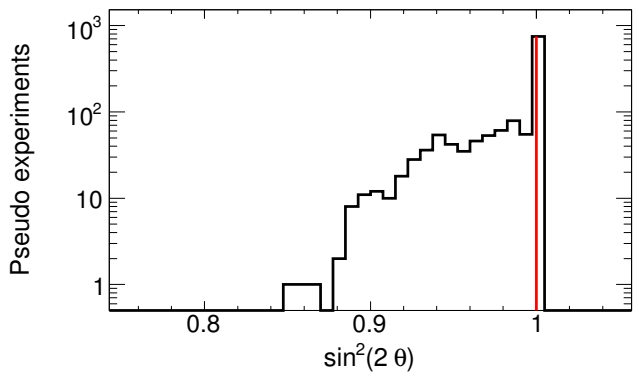

(c)

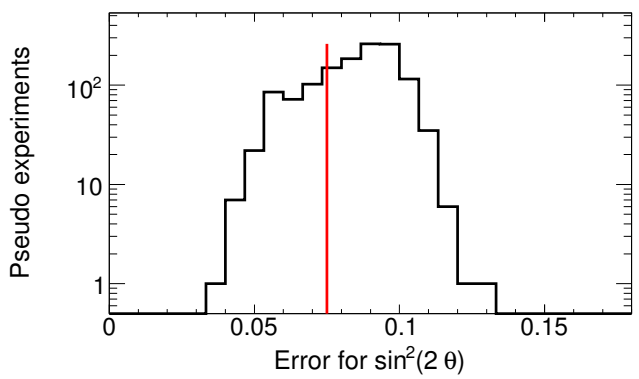

(e)

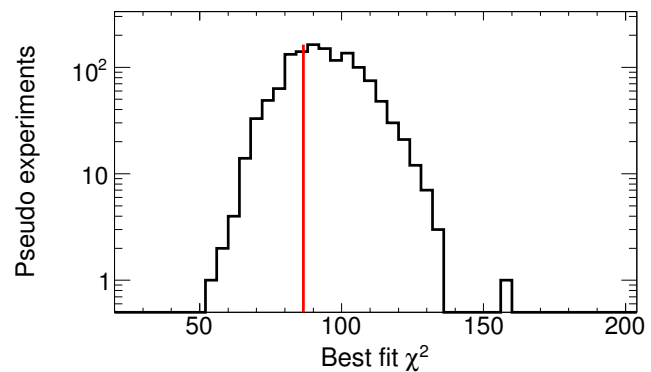

(b)

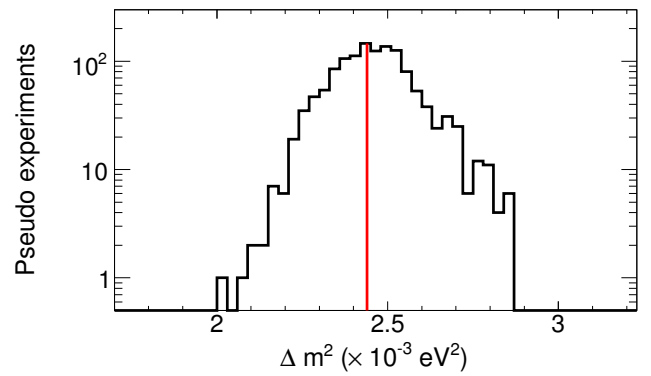

(d)

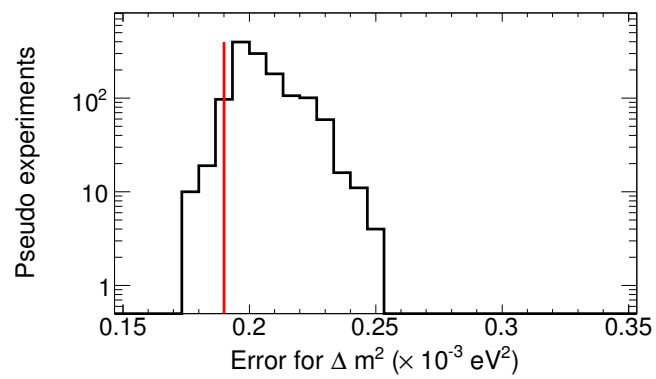

(f)

Figure 9.18: Results of 1200 independent pseudo-experiments for the uncorrelated far detector systematic errors, as discussed in the text. (a) the $\chi^{2}$ values for the data and MC without oscillations. (b) the $\chi^{2}$ values for the data and best oscillation fit MC. (c) and (d) the best fit oscillation parameters. (e) and (f) $90 \%$ C.L. statistical errors for the best fit parameters. Red lines show the values obtained from the oscillation fit to the real far detector data. 


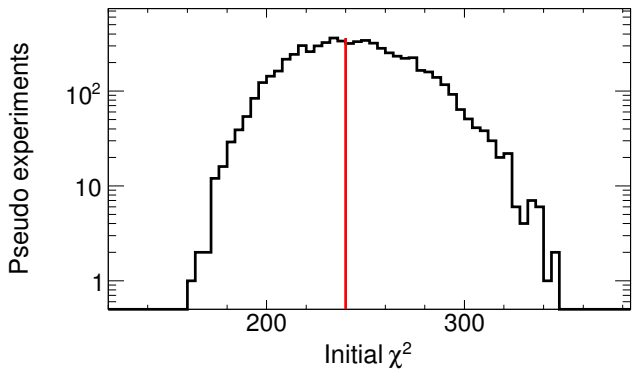

(a)

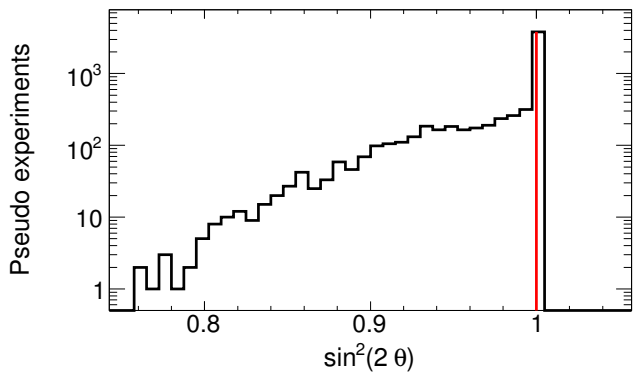

(c)

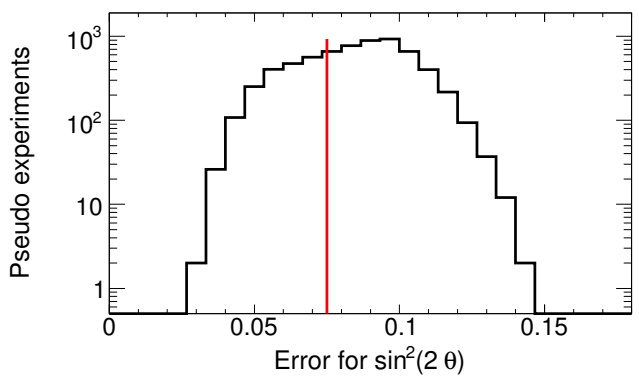

(e)

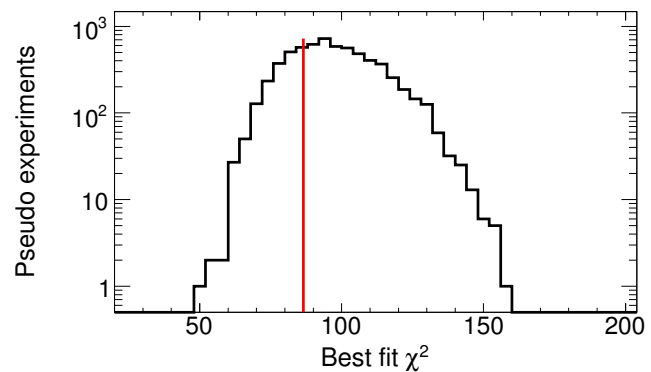

(b)

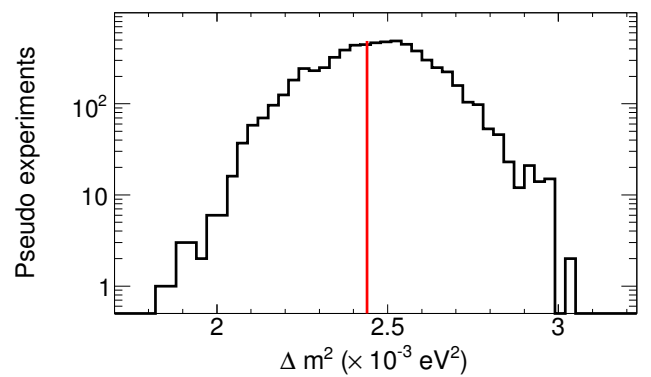

(d)

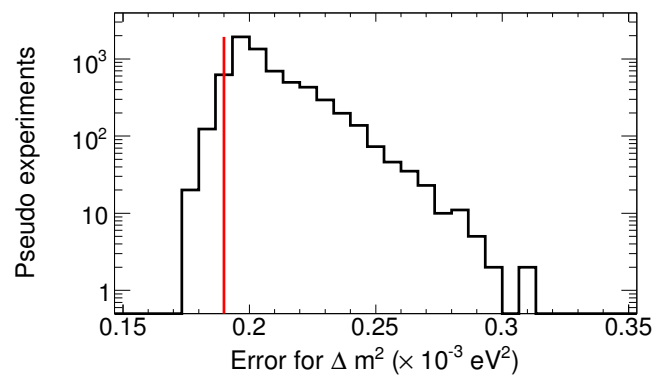

(f)

Figure 9.19: Results of 6400 independent pseudo-experiments for the correlated far detector systematic errors, as discussed in the text. (a) the $\chi^{2}$ values for the data and $\mathrm{MC}$ without oscillations. (b) the $\chi^{2}$ values for the data and best oscillation fit MC. (c) and (d) the best fit oscillation parameters. (e) and (f) $90 \%$ C.L. statistical errors for the best fit parameters. Red lines show the values obtained from the oscillation fit to the real far detector data. 


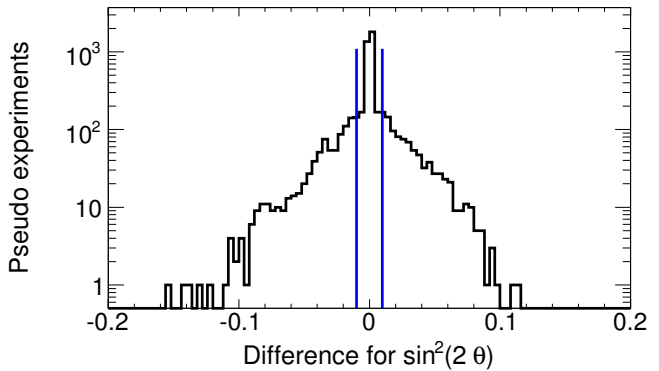

(a)

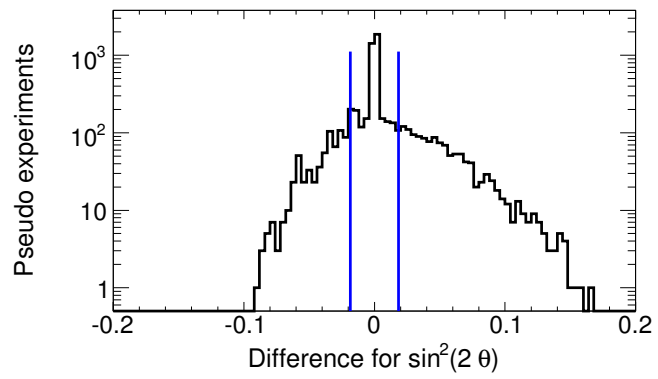

(c)

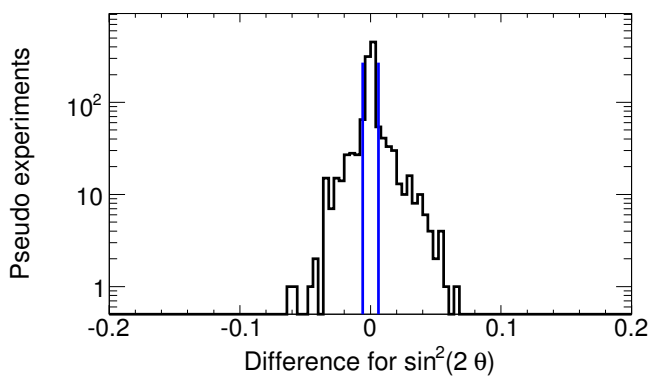

(e)

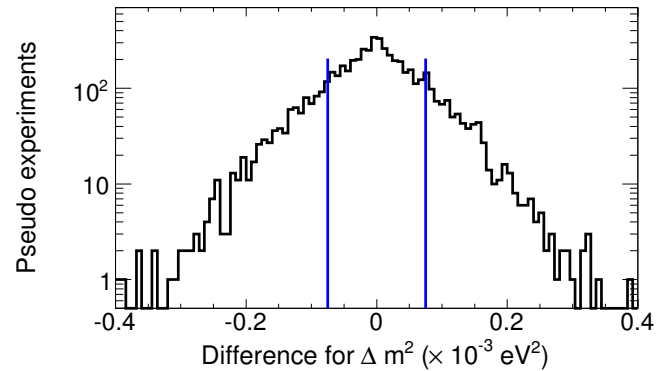

(b)

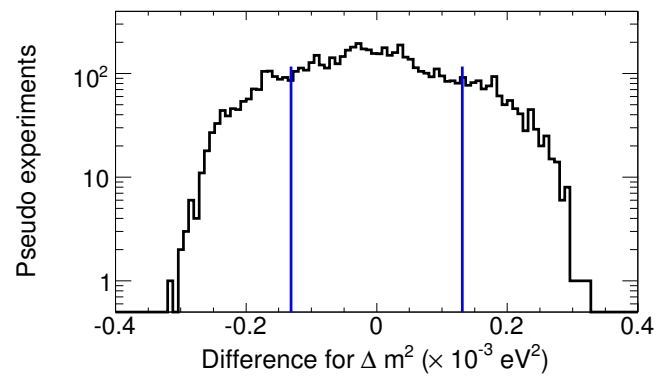

(d)

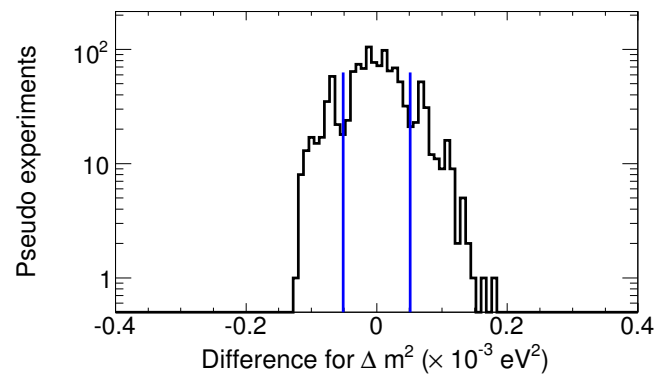

(f)

Figure 9.20: Systematic errors for the three sets of pseudo-experiments. Each figure shows a difference between two fit parameters. First fit parameter is obtained from a fit with a pseudo experiment without systematic effects. Second fit parameter is obtained from a fit with a pseudo experiment that includes one or more systematic shifts, as described in the text. In both cases, the same statistical pseudo experiment is used. Figures (a) and (b) show differences for the near detector systematic effects. Figures (c) and (d) show differences for the far detector correlated systematic effects. Figures (e) and (f) show differences for the far detector uncorrelated systematic effects. Blue lines mark a symmetric region that includes $68 \%$ of the pseudo-experiments. 


\subsection{MINOS results}

The oscillation results obtained in this dissertation are compared with the latest published result by the MINOS collaboration [46]. Four features distinguish this dissertation analysis. First, an improved approach to fitting the near detector data is used. Secondly, the oscillation parameters are obtained using separate histograms for the DIS events and the combination of the QES and RES events. Thirdly, a different procedure is employed to compute the systematic errors for the measurement of the oscillation parameters. Finally, the published results also include the data from the high energy beam configuration. The two analyses share the muon identification technique used to select $\nu_{\mu}$ charged-current events.

For the MINOS result, Figure 9.21 shows the reconstructed energy spectrum for the far detector data, the MC prediction without oscillations, and the MC spectrum weighted by the best fit oscillation parameters. The oscillation hypothesis gives an accurate description of the data. Figure 9.23 shows the allowed C.L. regions for the published MINOS result and the result presented in this dissertation. Two analyses are compatible and have measured the same values for the oscillation parameters. The systematic errors measured with the present analysis are larger than the errors for the MINOS analysis. The MINOS analysis included three largest systematic uncertainties into the oscillation fit as nuisance parameters; this procedure reduces the systematic errors. In addition, using multiple shifts to evaluate the systematic errors has the potential to produce the conservative systematic errors. 


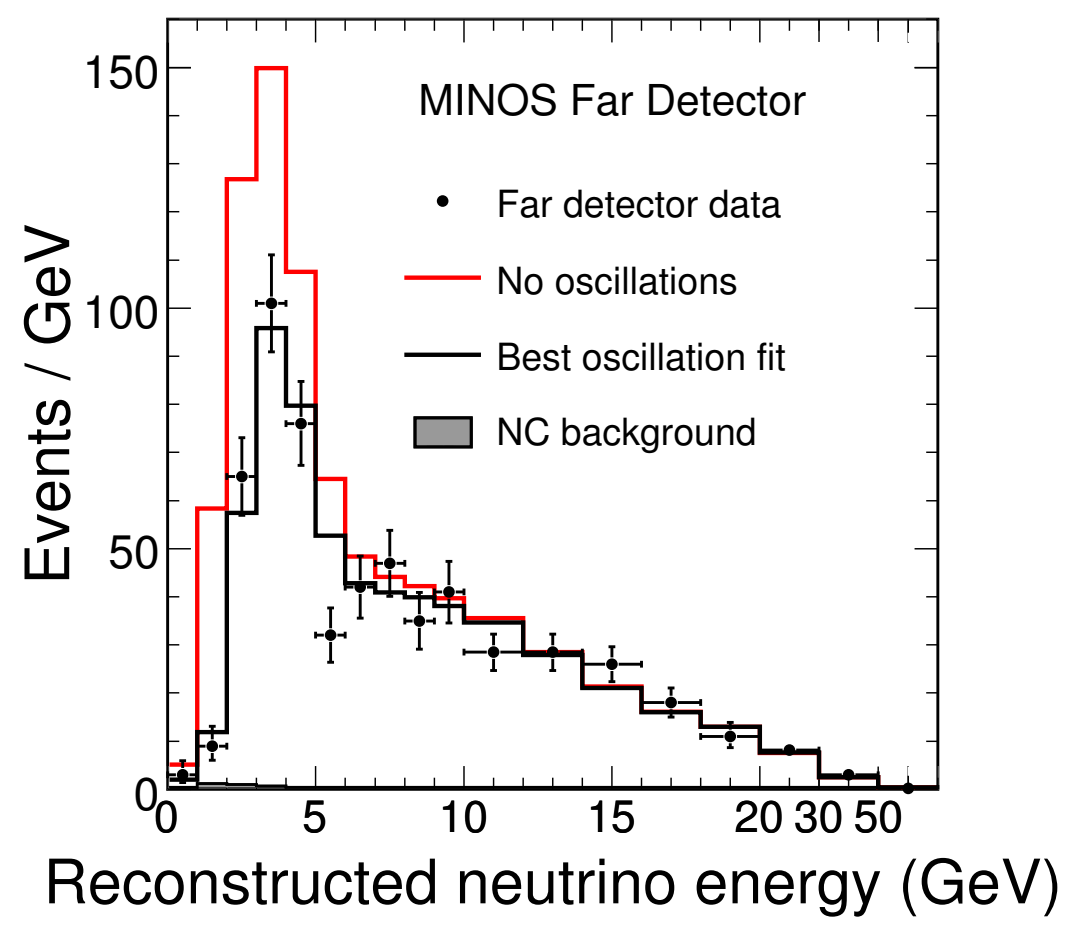

Figure 9.21: The latest published result by the MINOS collaboration for the measurement of oscillation parameters [46]. This figure shows the reconstructed energy spectra for the sum of the Run I and Run II events. Shown are the data events, the MC events without oscillations, and the MC events weighted by the best oscillation fit parameters. 


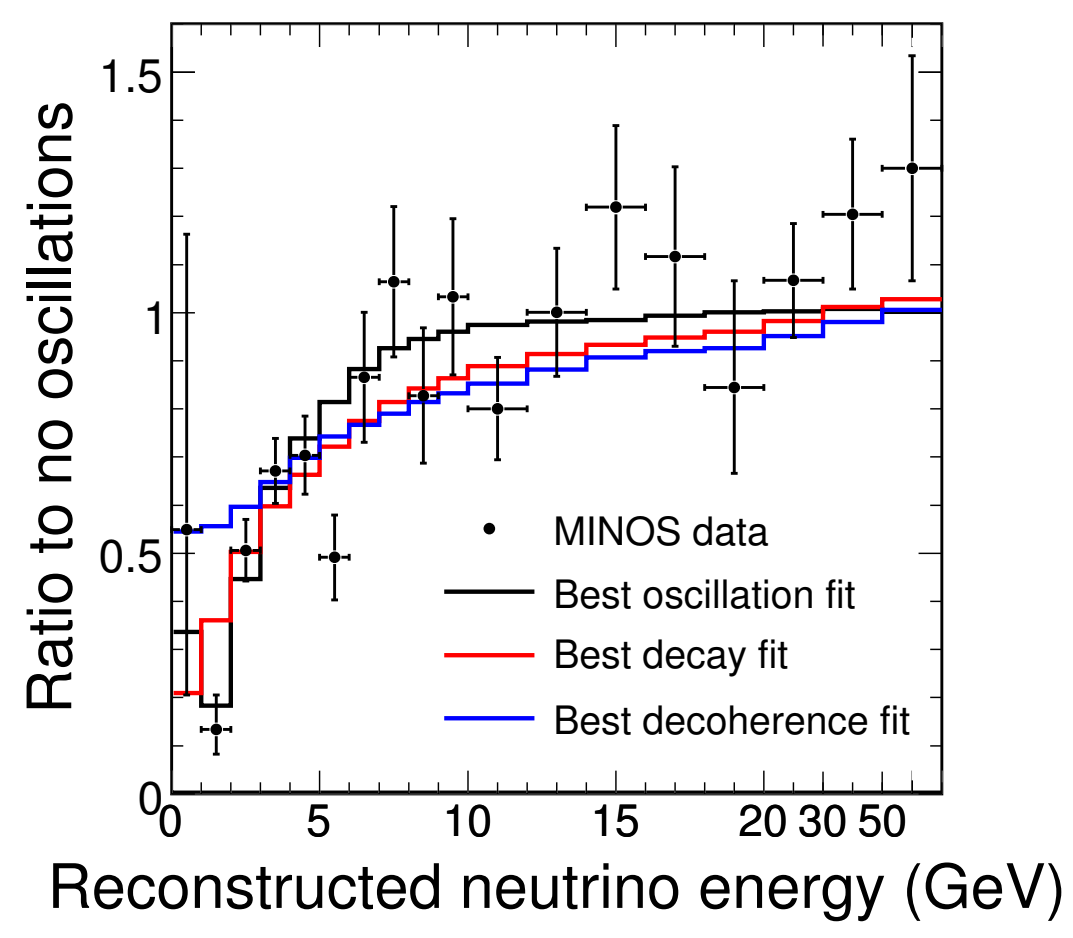

Figure 9.22: The latest published result by the MINOS collaboration for the measurement of oscillation parameters [46]. This figure shows the sum of the Run I and Run II events. This figure shows the data and MC simulation weighted by the best oscillation fit parameters divided the MC expectation without oscillations. These figures show the fits to the standard oscillation hypothesis and two alternative hypotheses (see Chapter 1). 


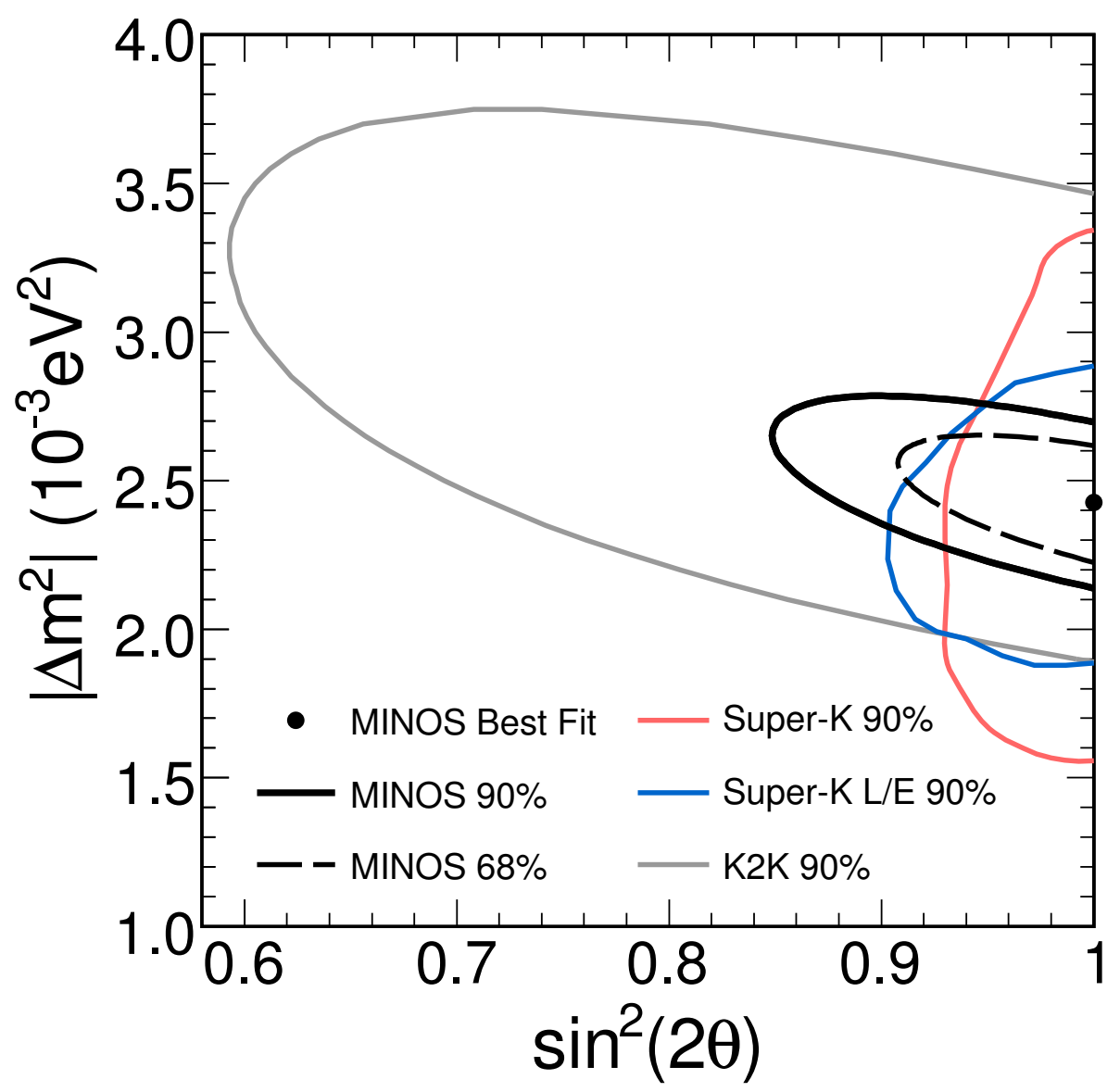

Figure 9.23: This figure shows $68 \%$ and $90 \%$ C.L. regions from the latest published result by the MINOS collaboration for the measurement of oscillation parameters [46]. The best fit parameters for the published MINOS result are: $\sin ^{2} 2 \theta>0.90\left(90 \%\right.$ C.L.) and $\left|\Delta m^{2}\right|=(2.43 \pm 0.13) \times 10^{-3} \mathrm{eV}^{2}$ (68\% C.L) (statical and systematic errors combined). The C.L. regions from Super-Kamiokanda [42] and K2K experiments [48] are also shown. 


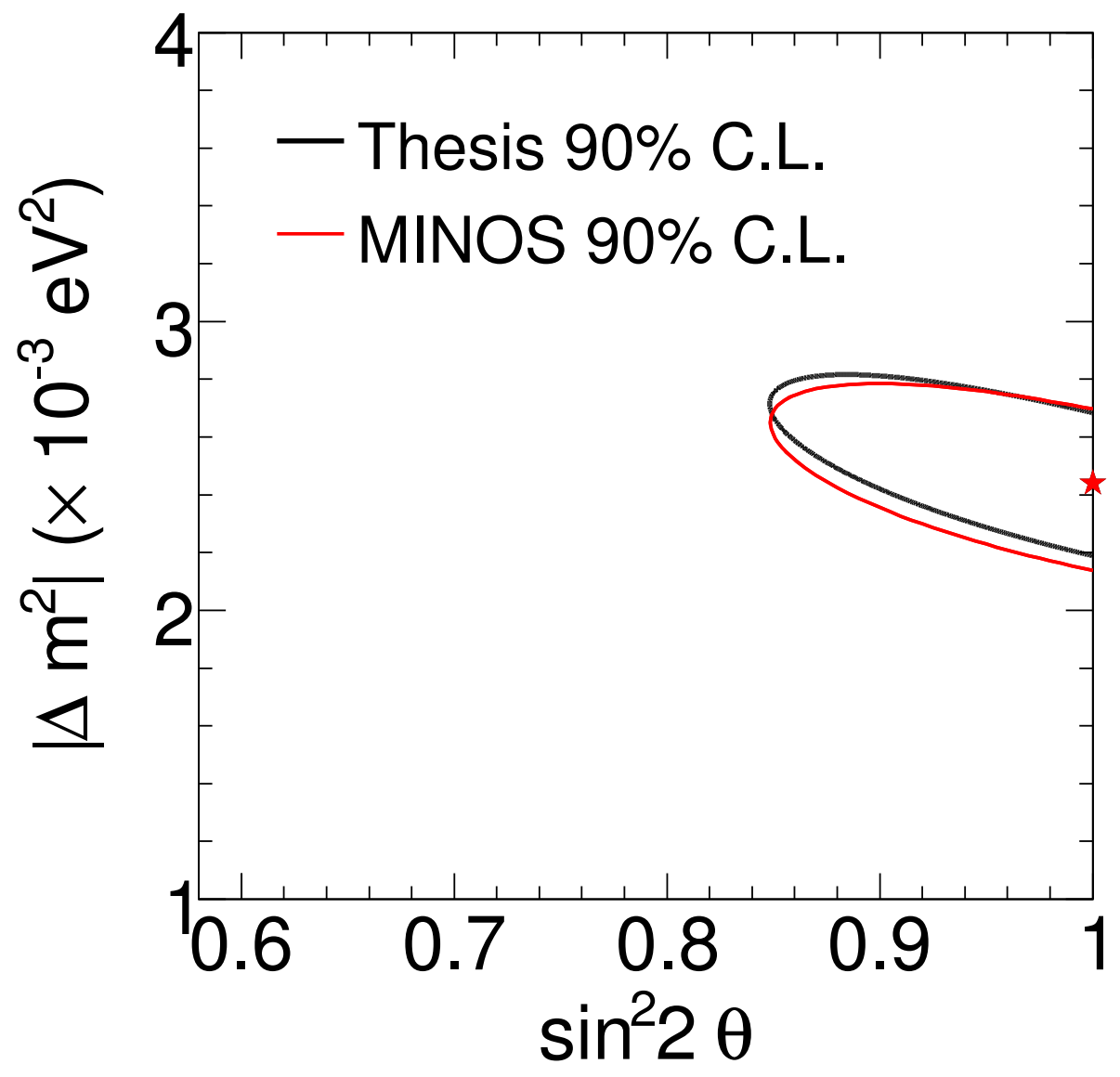

Figure 9.24: This figure shows 90\% C.L. regions for the analysis presented in this thesis and the published MINOS results [46]. The best fit parameters for the published MINOS result are: $\sin ^{2} 2 \theta>0.90$ (90\% C.L.) and $\left|\Delta m^{2}\right|=(2.43 \pm 0.13) \times 10^{-3} \mathrm{eV}^{2}(68 \%$ C.L) (statical and systematic errors combined) The best fit oscillation parameters for the thesis are: $\sin ^{2} 2 \theta>0.96$ $(68 \%$ C.L. $)$ and $\left|\Delta m^{2}\right|=(2.44 \pm 0.12) \times 10^{-3} \mathrm{eV}^{2}(68 \%$ C.L) (statistical errors only). The MINOS contour was obtained using the fit that includes the oscillation parameters as well as the systematic uncertainties, included as nuisance parameters. 


\subsection{Conclusions}

This thesis presented a complete analysis of the MINOS near and far detector data. The thesis developed techniques to identify the muon tracks and measure the muon charge sign in the MINOS detectors. The study of the neutrino interactions in the near detector led to the development of a simple method to identify quasi-elastic, resonance production, and deep inelastic scattering $\nu_{\mu}$ charged-current events. Also, an improved Monte-Carlo tuning procedure was presented. This procedure improved the agreement between the near detector data and the Monte-Carlo simulation for the quasi-elastic, resonance production, and deep inelastic scattering $\nu_{\mu}$ charged-current events. The neutrino flux and cross-section parametrization were used to generate the predicted far detector Monte-Carlo events without neutrino oscillations. The far detector data showed an energy dependent deficit when compared to the Monte-Carlo prediction without oscillations. The fit to the far detector data was performed using the oscillation hypothesis for the two massive neutrinos. The fit gives an excellent description of the data. The measured oscillation parameters are:

$$
\begin{aligned}
& \sin ^{2} 2 \theta=1.0_{-0.038}(\text { stat. })_{-0.022} \text { (syst.) }(68 \% \text { C.L. }) \\
& \left.\left|\Delta m^{2}\right|=2.44_{-0.12}^{+0.12} \text { (stat. }\right)_{-0.16}^{+0.16} \text { (syst.) } \times 10^{-3} \mathrm{eV}^{2}(68 \% \text { C.L. })
\end{aligned}
$$


Appendices 


\section{Appendix A}

\section{Summary of kinematic distributions}

This chapter compares the near detector data with the default and tuned MC simulation. These comparisons use the reconstructed energy spectra for several values of the Bjorken scaling variable, $X$, and the momentum transfered squared variable, $Q^{2}$. In this chapter, all figures show the selected $\nu_{\mu}$ charged-current events. The data events were recorded during the Run II period, using the low energy beam configuration. The $X$ and $Q^{2}$ variables are defined in Chapter 3. The event selection procedure is summarized in Chapter 6 . The default and tuned MC simulations are described in Chapter 7 . In all figures, the data events are shown as black points; the default MC events are shown with a blue line; and, the tuned MC events are shown with a red line. Selected ranges of the $X$ and $Q^{2}$ variables are labeled individually on each figure. The tuned MC histograms include statistical and systematic errors; these errors are discussed in Chapter 6. Right hand panels show the data spectra divided by the tuned and default MC spectra.

Before tuning, there are approximately $20 \%$ more data events with energy less than $4 \mathrm{GeV}$. These differences between the data and default MC simulation depend on the $X$ and $Q^{2}$ variables. This fact suggests that in this 
region the neutrino cross-sections are not correctly modeled.

There are also approximately $40 \%$ more data events with energy greater than $4 \mathrm{GeV}$, compared with the default MC simulation. These disagreements depend neither on the Bjorken scaling variable, $X$, nor the momentum transfered squared variable, $Q^{2}$. This fact suggests that these differences are caused by an incorrect modeling of the NuMI neutrino flux, as demonstrated by the previous analysis $[58,101]$.

The tuned MC simulation accurately describes the data, except for the low $X$ and the low $Q^{2}$ values. 

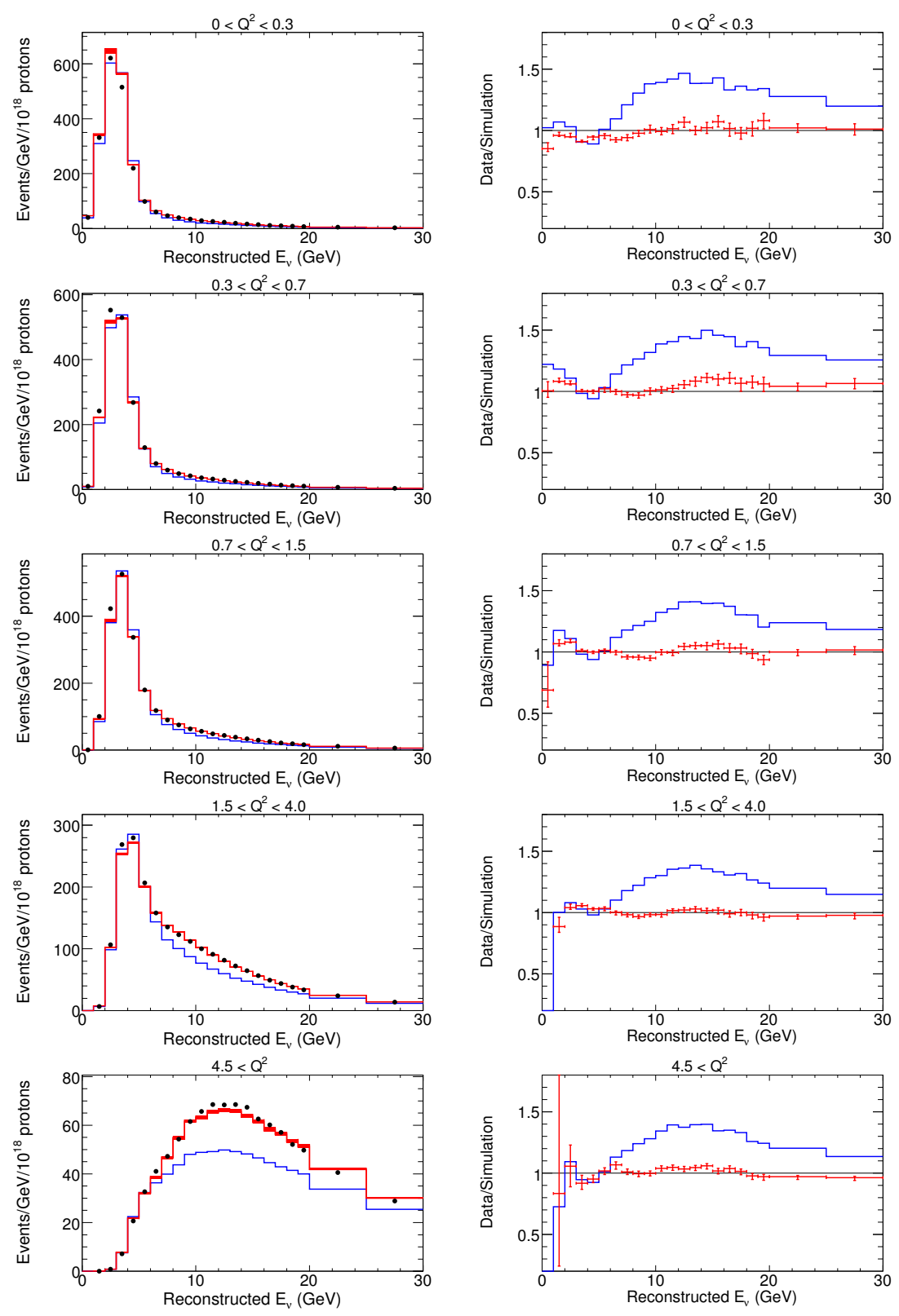

Figure A.1: The $E_{\nu}$ spectra for several values of $Q^{2}$. 

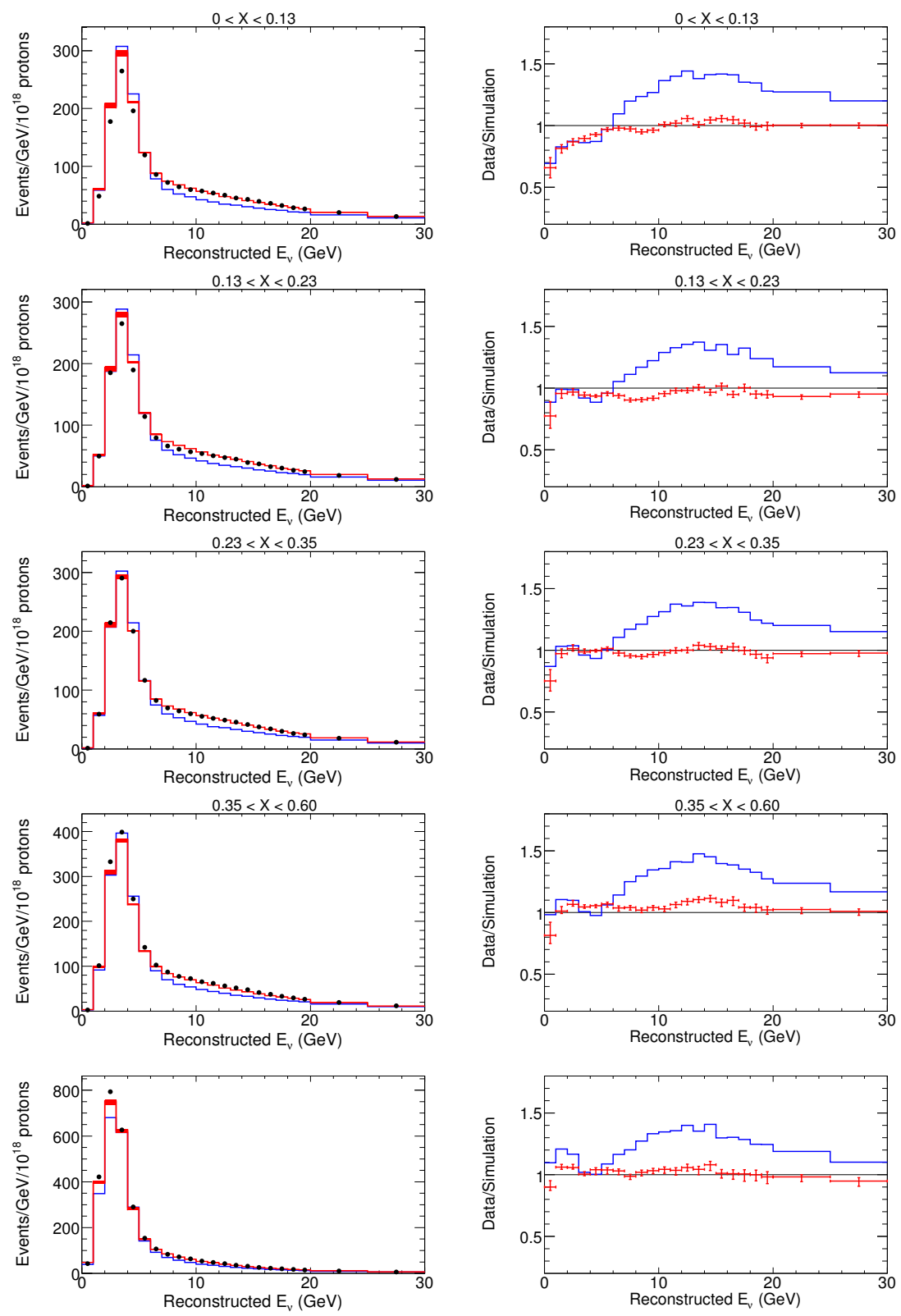

Figure A.2: The $E_{\nu}$ spectra for several values of $X$. 

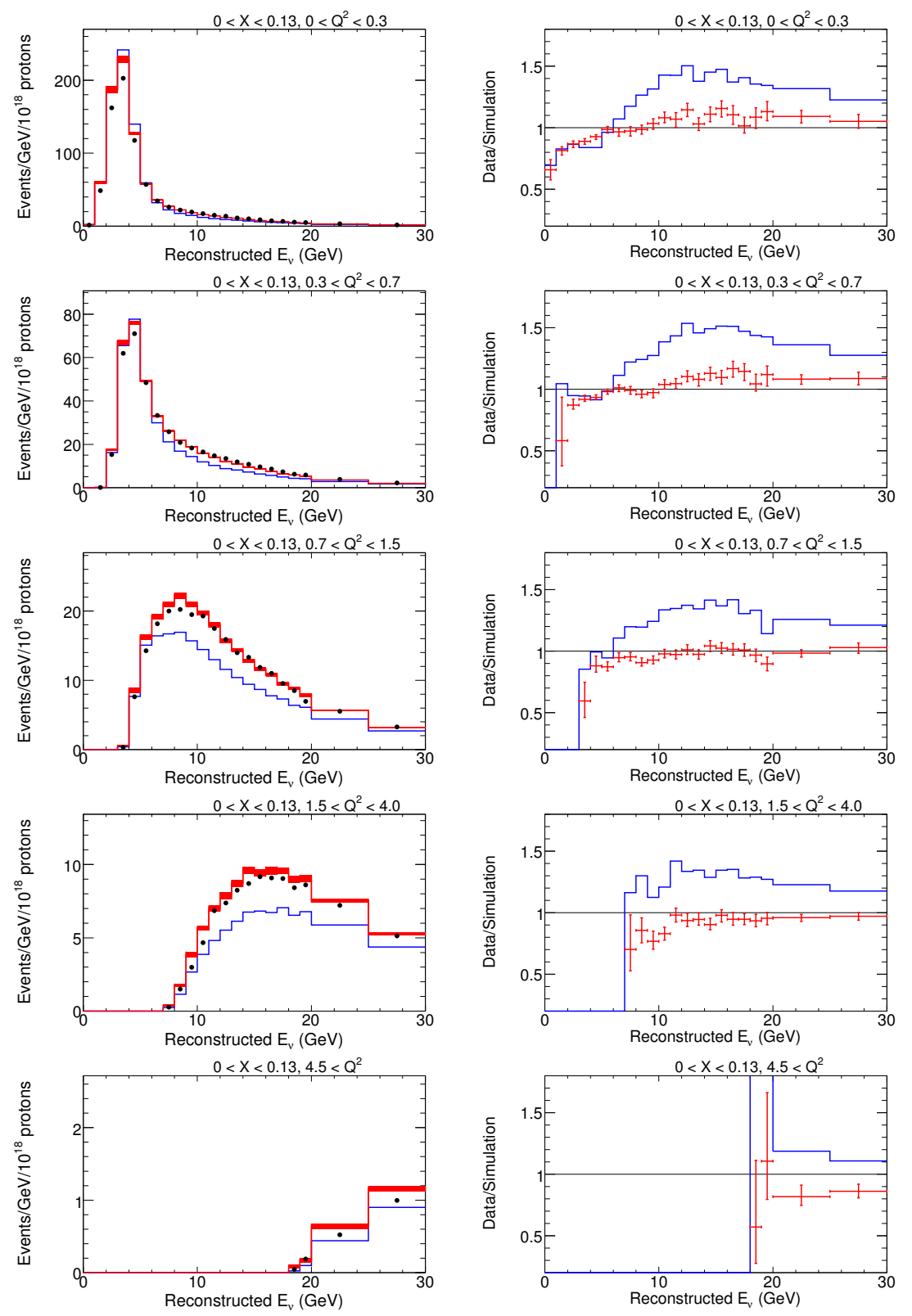

Figure A.3: The $E_{\nu}$ spectra for several values of $Q^{2}$. The $X$ variable is constrained within this range: $0<X<0.13$. 

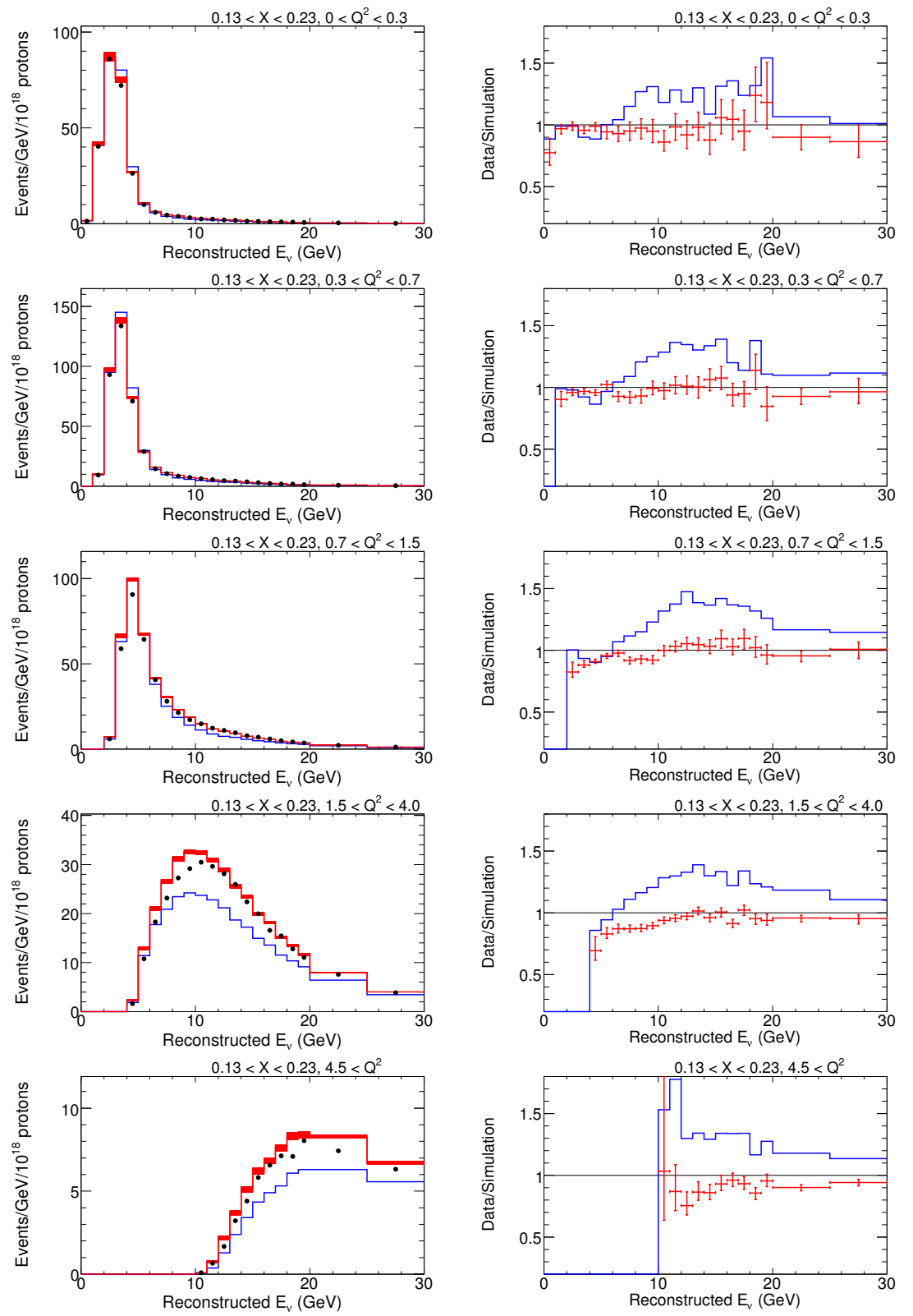

Figure A.4: The $E_{\nu}$ spectra for several values of $Q^{2}$. The $X$ variable is constrained within this range: $0.13<X<0.23$. 

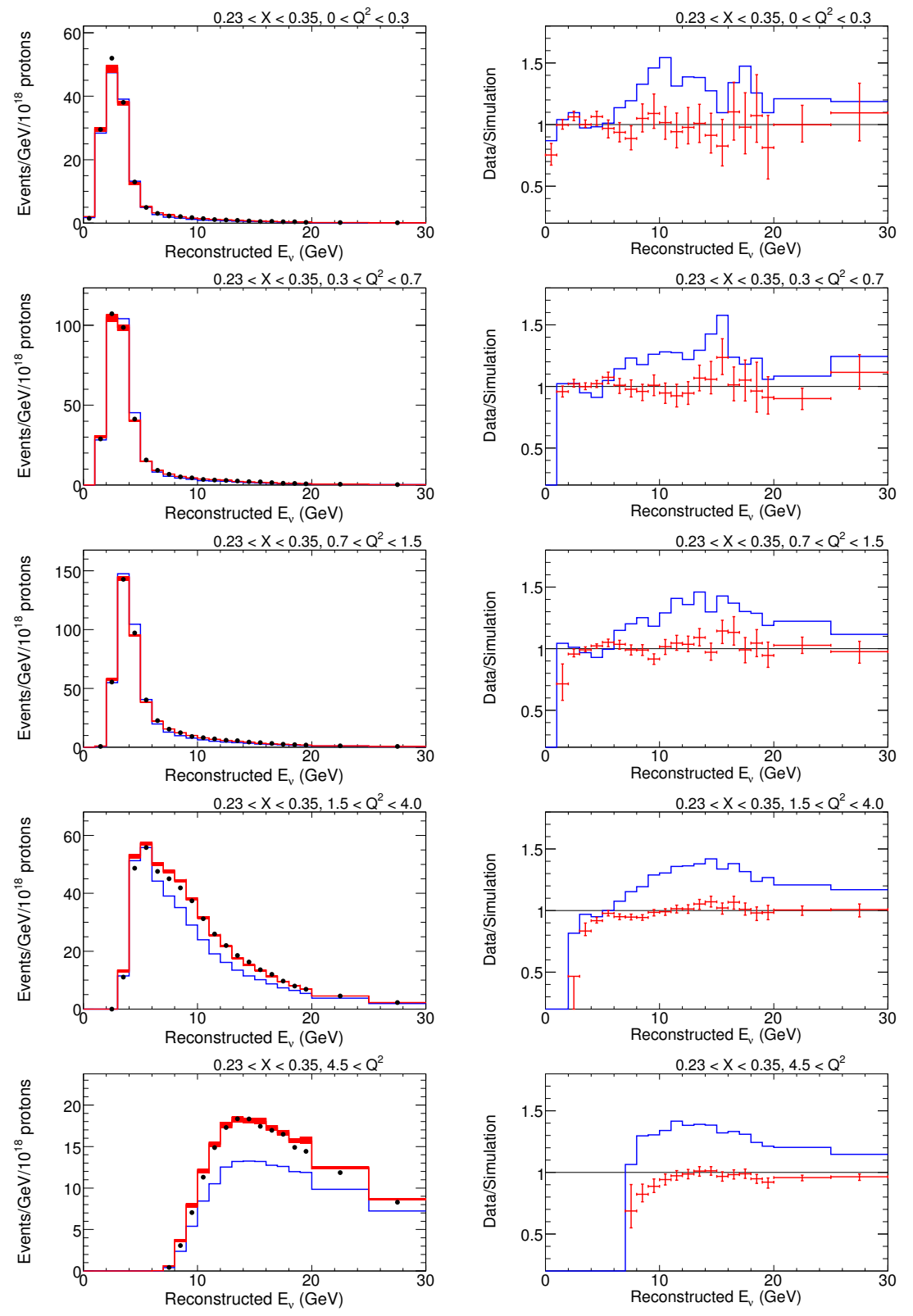

Figure A.5: The $E_{\nu}$ spectra for different values of $Q^{2}$. The $X$ variable is constrained within this range: $0.23<X<0.35$. 

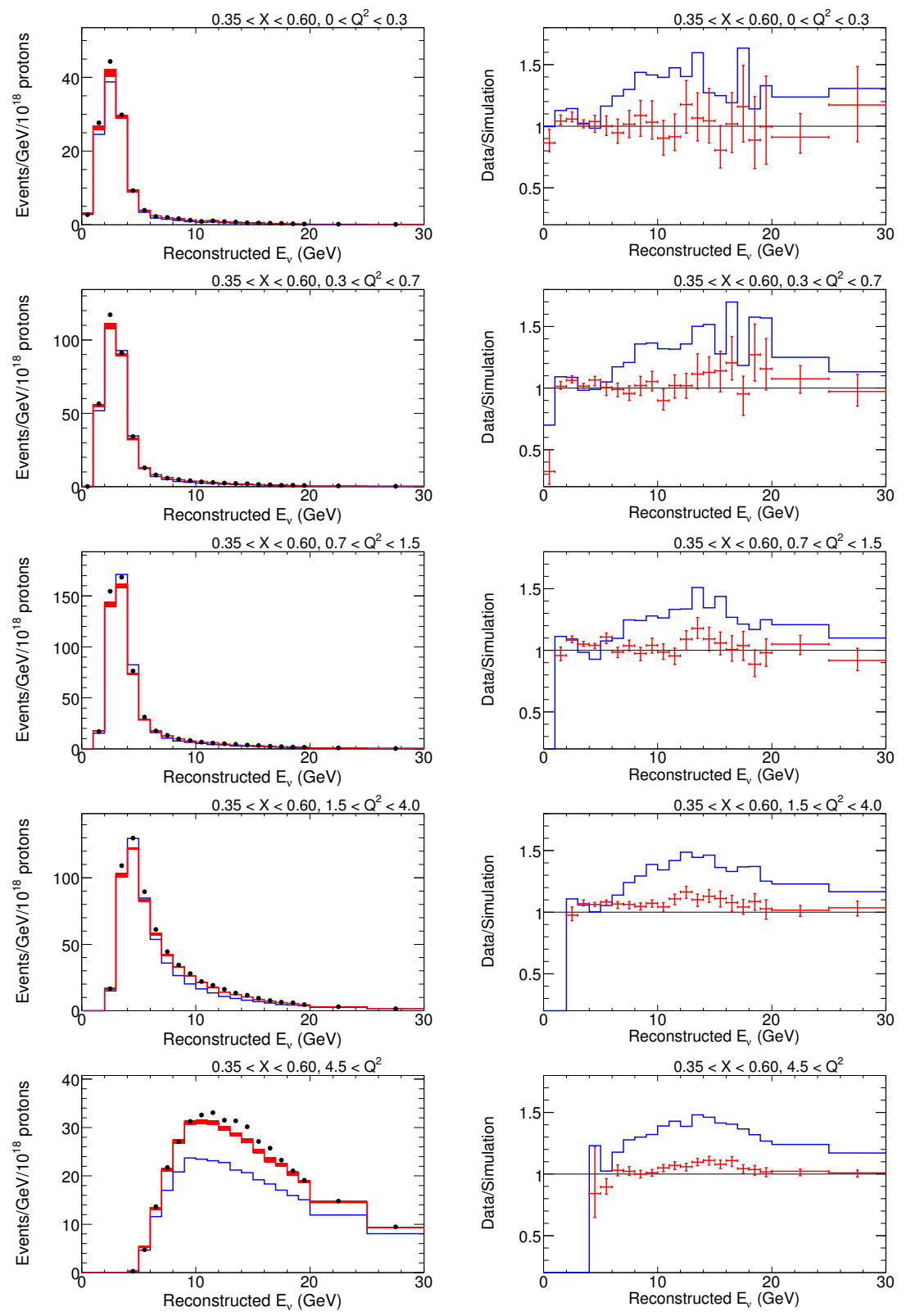

Figure A.6: The $E_{\nu}$ spectra for different values of $Q^{2}$. The $X$ variable is constrained within this range: $0.36<X<0.6$. 

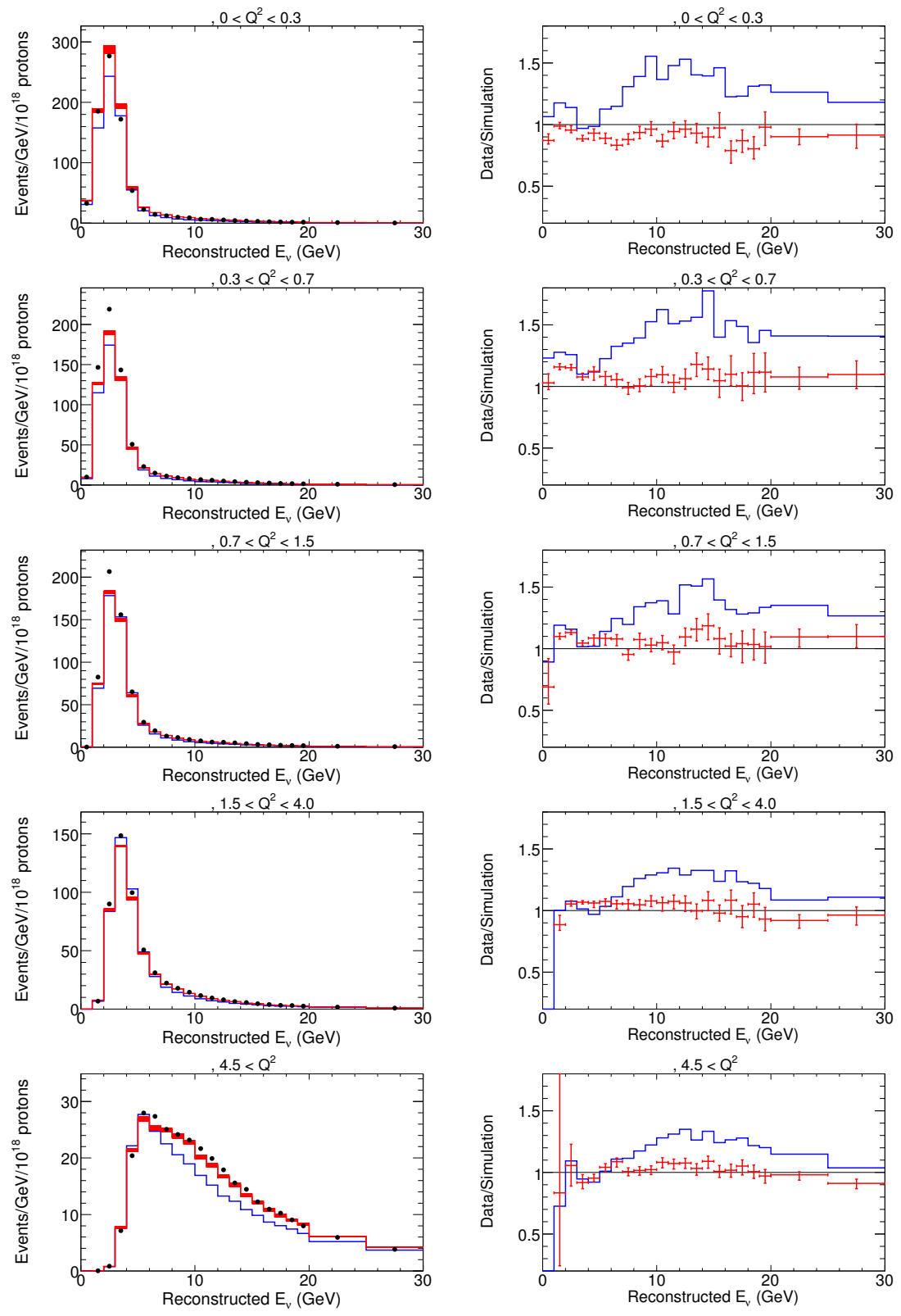

Figure A.7: The $E_{\nu}$ spectra for different values of $Q^{2}$. The $X$ variable is constrained within this range: $X>0.6$. 


\section{Bibliography}

[1] J. Chadwick, Verh. Deutsch. Phys. Ges., 16, 383 (1914).

[2] C. D. Ellis and W. A. Wooster, The Average Energy of Disintegration of Radium E, Proceedings of the Royal Society of London Series A, 117 (776), 109-123 (1927).

[3] J. Chadwick, Possible Existence Of A Neutron, Nature, 129, 312 (1932).

[4] E. Fermi, An attempt of a theory of beta radiation. 1, Z. Phys., 88, 161-177 (1934).

[5] C. Amsler et al., Review of particle physics, Phys. Lett., B667, 1 (2008).

[6] C. L. Cowan, F. Reines, F. B. Harrison, H. W. Kruse and A. D. McGuire, Detection of the Free Neutrino: a Confirmation, Science, 124 (3212), 103-104 (1956).

[7] B. Pontecorvo, Chalk River Nat. Lab. Report PD-205 (1946).

[8] L. Alvarez, University of California Rad. Lab Report UCRL-328 (1949).

[9] J. Davis, Raymond, D. S. Harmer and K. C. Hoffman, Search for neutrinos from the sun, Phys. Rev. Lett., 20, 1205-1209 (1968). 
[10] G. Danby et al., Observation of high-energy neutrino reactions and the existence of two kinds of neutrinos, Phys. Rev. Lett., 9, 36-44 (1962).

[11] J. J. Aubert et al., Experimental Observation of a Heavy Particle J, Phys. Rev. Lett., 33, 1404-1406 (1974).

[12] J. E. Augustin et al., Discovery of a Narrow Resonance in e+ e- Annihilation, Phys. Rev. Lett., 33, 1406-1408 (1974).

[13] F. J. Hasert et al., Observation of neutrino-like interactions without muon or electron in the Gargamelle neutrino experiment, Phys. Lett., B46, 138-140 (1973).

[14] S. W. Herb et al., Observation of a dimuon resonance at $9.5-\mathrm{GeV}$ in 400-GeV proton - nucleus collisions, Phys. Rev. Lett., 39, 252-255 (1977).

[15] M. L. Perl et al., Evidence for anomalous lepton production in e+ eannihilation, Phys. Rev. Lett., 35, 1489-1492 (1975).

[16] G. Arnison et al., Experimental observation of lepton pairs of invariant mass around $95-\mathrm{GeV} / \mathrm{c}^{* *} 2$ at the CERN SPS collider, Phys. Lett., B126, 398-410 (1983).

[17] P. Bagnaia et al., Evidence for $Z 0-->e+e-$ at the CERN anti-p p collider, Phys. Lett., B129, 130-140 (1983). 
[18] F. Abe et al., Observation of top quark production in $\bar{p} p$ collisions, Phys. Rev. Lett., 74, 2626-2631 (1995).

[19] K. Kodama et al., Observation of tau-neutrino interactions, Phys. Lett., B504, 218-224 (2001).

[20] B. Pontecorvo, Mesonium and antimesonium, Sov. Phys. JETP, 6, 429 (1957).

[21] B. Pontecorvo, Inverse beta processes and nonconservation of lepton charge, Sov. Phys. JETP, 7, 172-173 (1958).

[22] Z. Maki, M. Nakagawa and S. Sakata, Remarks on the unified model of elementary particles, Prog. Theor. Phys., 28, 870 (1962).

[23] S. M. Bilenky, C. Giunti and W. Grimus, Phenomenology of neutrino oscillations, Prog. Part. Nucl. Phys., 43, 1-86 (1999).

[24] B. Kayser, On the Quantum Mechanics of Neutrino Oscillation, Phys. Rev., D24, 110 (1981).

[25] L.-L. Chau and W.-Y. Keung, Comments on the Parametrization of the Kobayashi-Maskawa Matrix, Phys. Rev. Lett., 53, 1802 (1984).

[26] R. Davis, Solar neutrinos. II: Experimental, Phys. Rev. Lett., 12, 303-305 (1964). 
[27] J. N. Bahcall, A. M. Serenelli and S. Basu, New solar opacities, abundances, helioseismology, and neutrino fluxes, Astrophys. J., 621, L85L88 (2005).

[28] J. N. Bahcall, Solar neutrinos. I: Theoretical, Phys. Rev. Lett., 12, 300-302 (1964).

[29] J. Hosaka et al., Solar neutrino measurements in Super-Kamiokande-I, Phys. Rev., D73, 112001 (2006), hep-ex/0508053.

[30] B. Aharmim et al., An Independent Measurement of the Total Active 8B Solar Neutrino Flux Using an Array of 3He Proportional Counters at the Sudbury Neutrino Observatory, Phys. Rev. Lett., 101, 111301 (2008).

[31] S. Fukuda et al., Determination of solar neutrino oscillation parameters using 1496 days of Super-Kamiokande-I data, Phys. Lett., B539, 179187 (2002), hep-ex/0205075.

[32] B. Aharmim et al., Electron energy spectra, fluxes, and day-night asymmetries of B-8 solar neutrinos from the 391-day salt phase SNO data set, Phys. Rev., C72, 055502 (2005).

[33] B. Pontecorvo, Neutrino experiments and the question of leptonic-charge conservation, Sov. Phys. JETP, 26, 984-988 (1968).

[34] V. N. Gribov and B. Pontecorvo, Neutrino astronomy and lepton charge, Phys. Lett., B28, 493 (1969). 
[35] L. Wolfenstein, Neutrino oscillations in matter, Phys. Rev., D17, 2369 2374 (1978).

[36] S. Abe et al., Precision Measurement of Neutrino Oscillation Parameters with KamLAND, Phys. Rev. Lett., 100, 221803 (2008).

[37] Y. Declais et al., Search for neutrino oscillations at 15-meters, 40-meters, and 95-meters from a nuclear power reactor at Bugey, Nucl. Phys., B434, 503-534 (1995).

[38] F. Boehm et al., Final results from the Palo Verde neutrino oscillation experiment, Phys. Rev., D64, 112001 (2001).

[39] M. Apollonio et al., Search for neutrino oscillations on a long base-line at the CHOOZ nuclear power station, Eur. Phys. J., C27, 331-374 (2003), hep-ex/0301017.

[40] P. F. Harrison, D. H. Perkins and W. G. Scott, Further evidence for threefold maximal lepton mixing and a hierarchical spectrum of neutrino mass-squared differences, Phys. Lett., B396, 186-196 (1997).

[41] W. A. Mann, Atmospheric neutrinos and the oscillations bonanza (1999), arXiv: hep-ex/9912007.

[42] Y. Ashie et al., Evidence for an oscillatory signature in atmospheric neutrino oscillation, Phys. Rev. Lett., 93, 101801 (2004). 
[43] Y. Fukuda et al., Study of the atmospheric neutrino flux in the multiGeV energy range, Phys. Lett., B436, 33-41 (1998).

[44] V. D. Barger, J. G. Learned, S. Pakvasa and T. J. Weiler, Neutrino decay as an explanation of atmospheric neutrino observations, Phys. Rev. Lett., 82, 2640-2643 (1999).

[45] G. L. Fogli, E. Lisi, A. Marrone and D. Montanino, Status of atmospheric $\mathrm{nu} / \mathrm{mu}-i \mathrm{nu} /$ tau oscillations and decoherence after the first K2K spectral data, Phys. Rev., D67, 093006 (2003).

[46] P. Adamson et al., Measurement of Neutrino Oscillations with the MINOS Detectors in the NuMI Beam, Phys. Rev. Lett., 101, 131802 (2008).

[47] S. E. Kopp, Accelerator neutrino beams, Phys. Rept., 439, 101-159 (2007).

[48] M. H. Ahn et al., Measurement of neutrino oscillation by the K2K experiment, Phys. Rev., D74, 072003 (2006).

[49] R. Acquafredda et al., First events from the CNGS neutrino beam detected in the OPERA experiment, New J. Phys., 8, 303 (2006).

[50] Y. Hayato, T2K at J-PARC, Nucl. Phys. Proc. Suppl., 143, 269-276 (2005). 
[51] R. P. Litchfield, Neutrino induced events in the MINOS detectors, Ph.D. thesis, University of Oxford (2008).

[52] D. G. Michael et al., The magnetized steel and scintillator calorimeters of the MINOS experiment, Nucl. Instrum. Meth., A596, 190-228 (2008).

[53] K. Anderson et al., The NuMI Facility Technical Design Report (1998).

[54] B. Pontecorvo, Electron and muon neutrinos, Sov. Phys. JETP, 10, 1236-1240 (1960).

[55] M. Schwartz, Feasibility of using high-energy neutrinos to study the weak interactions, Phys. Rev. Lett., 4, 306-307 (1960).

[56] S. van der Meer, A directive device for charged particles and its use in an enhanced neutrino beam, CERN-61-07 (1961).

[57] S. E. Kopp, The NuMI neutrino beam at Fermilab (2005), arXiv: physics/0508001.

[58] P. Adamson et al., A Study of Muon Neutrino Disappearance Using the Fermilab Main Injector Neutrino Beam, Phys. Rev., D 77, 072002 (2008).

[59] L. Loiacono and S. Kopp, Beam quality cuts, MINOS Document 2747 (2007).

[60] A. Fasso et al., The physics models of FLUKA: Status and recent development (2003), arXiv:hep-ph/0306267. 
[61] A. Ferrari, P. R. Sala, A. Fasso and J. Ranft, FLUKA: A multi-particle transport code (Program version 2005), CERN-2005-010.

[62] Application Software Group, GEANT Detector Description and Simulation Tool, CERN Program Library Long Writeup W5013.

[63] R. Armstrong et al., Constraining Hadron Production Spectra in NuMI Beam by A Fit to The MINOS Near Detector Data, MINOS Document 4166 (2008).

[64] P. Adamson et al., The MINOS calibration detector, Nucl. Instrum. Meth., A556, 119-133 (2006).

[65] A. Cabrera, Systematic Comparison of the MINOS Near and Far Detector Readout Systems, Ph.D. thesis, University of Oxford (2005).

[66] T. Cundiff et al., The MINOS near detector front end electronics, IEEE Trans. Nucl. Sci., 53, 1347-1355 (2006).

[67] J. Oliver, N. Felt, G. Feldman, A. Lebedev and R. Lee, Design and performance of the readout system of the MINOS Far Detector, IEEE Trans. Nucl. Sci., 51, 2193-2195 (2004).

[68] A. Belias et al., The MINOS data acquisition system, IEEE Trans. Nucl. Sci., 51, 451-455 (2004).

[69] P. Adamson et al., The MINOS light injection calibration system, Nucl. Instrum. Meth., A492, 325-343 (2002). 
[70] J. J. Hartnell, Measurement of the MINOS Detectors' Relative Calorimetric Energy Response, Ph.D. thesis, University of Oxford (2005).

[71] P. A. Symes, Preliminary measurement of neutrino oscillation parameters by NuMI/MINOS and calibration studies for improving this measurement, Ph.D. thesis, University of Sussex (2005).

[72] M. A. Kordosky, Hadronic Interactions in the MINOS Detectors, Ph.D. thesis, University of Texas (2004).

[73] P. L. Vahle, Electromagnetic Interactions in the MINOS Detectors, Ph.D. thesis, University of Texas (2004).

[74] J. S. Marshall, A study of muon neutrino disappearance with the MINOS detectors and the NuMI neutrino beam, Ph.D. thesis, Cambridge University (2008).

[75] D. E. Groom, N. V. Mokhov and S. I. Striganov, Muon stopping power and range tables 10-mev to 100-tev, Atom. Data Nucl. Data Tabl., 78, $183-356$ (2001).

[76] T. H. Osiecki, A search for sterile neutrinos in MINOS, Ph.D. thesis, University of Texas (2007).

[77] M. A. Thomson, Alternative DeMuxer, MINOS Document 2535 (2006).

[78] H. Gallagher, The NEUGEN neutrino event generator, Nucl. Phys. B Proc. Suppl., 112, 188-194 (2002). 
[79] Durham Database Group, HEPDATA: Reaction Data Database.

[80] M. Dorman, Cross Section Measurements for Quasi-Elastic NeutrinoNucleus Scattering with the MINOS Near Detector, Ph.D. thesis, University College London (2008).

[81] G. P. Zeller, Low energy neutrino cross sections: Comparison of various Monte Carlo predictions to experimental data (2003), arXiv:hep-ex/ 0312061.

[82] J. M. Conrad, M. H. Shaevitz and T. Bolton, Precision measurements with high energy neutrino beams, Rev. Mod. Phys., 70, 1341-1392 (1998).

[83] T. K. Gaisser and J. S. O'Connell, Interactions of atmospheric neutrinos in nuclei at low-energy, Phys. Rev., D34, 822-825 (1986).

[84] A. Bodek and J. L. Ritchie, Fermi Motion Effects in Deep Inelastic Lepton Scattering from Nuclear Targets, Phys. Rev., D23, 1070 (1981).

[85] R. D. Ransome, Pion absorption and re-scattering, Nucl. Phys. B Proc. Suppl., 139, 208-212 (2005).

[86] M. Kordosky, Ramifications of intranuclear re-scattering in MINOS, Nucl. Phys. B Proc. Suppl., 159, 223-228 (2006).

[87] S. Dytman, H. Gallagher and M. Kordosky, Hadronic Shower Energy Scale Uncertainty in the MINOS Experiment (2008), arXiv:hep-ex/ 0806.2119 
[88] L. Landau, On the energy loss of fast particles by ionization, J. Phys. (USSR), 8, 201-205 (1944).

[89] R. Ospanov, Muon energy straggling function and the near detector dE/dx, MINOS Document 2637 (2007).

[90] R. Ospanov and K. Lang, Selecting charged current $\nu_{\mu}$ events in the data and the simulation, MINOS Document 4390 (2008).

[91] E. Barnes and N. Saoulidou, Low Pulse Height excess in the Near Detector, MINOS Document 2337 (2006).

[92] N. Tagg et al., Performance of Hamamatsu 64-anode photomultipliers for use with wavelength-shifting optical fibres, Nucl. Instrum. Meth., A539, 668-678 (2005).

[93] K. Lang et al., Characterization of 1600 Hamamatsu 16-anode photomultipliers for the MINOS Far detector, Nucl. Instrum. Meth., A545, 852-871 (2005).

[94] D. G. Michael et al., Observation of muon neutrino disappearance with the MINOS detectors and the NuMI neutrino beam, Phys. Rev. Lett., 97, 191801 (2006).

[95] R. Ospanov, CC selection with k-nearest neighbor algorithm presentation, MINOS Document 2740 (2007). 
[96] T. Hastie, R. Tibshirani and J. Friedman, The Elements of Statistical Learning: Data Mining, Inference, and Prediction, Springer (2001).

[97] A. Hocker et al., TMVA: Toolkit for multivariate data analysis, PoS, ACAT, 040 (2007), arXiv:physics/0703039.

[98] J. L. Bentley, Multidimensional binary search trees used for associative searching, Commun. ACM, 18 (9), 509-517 (1975).

[99] J. H. Freidman, J. L. Bentley and R. A. Finkel, An Algorithm for Finding Best Matches in Logarithmic Expected Time, ACM Trans. Math. Softw., 3 (3), 209-226 (1977).

[100] D. Petyt, PID sensitivity study status, MINOS Document 2743 (2007).

[101] Z. Pavlovic, Observation of muon neutrino disappearance in the NuMI beam, Ph.D. thesis, University of Texas (2008).

[102] D. T. J. Thomas, A. Para, The Principles of Muon Tracking in MINOS, MINOS Document 301 (1997).

[103] J. Hartnell, Details of the ND and FD Fiducial Volume for CC and NuMuBar Analyses, MINOS Document 4099 (2008).

[104] D. A. Petyt, Description of the ND fit method for the MINOS $\nu_{\mu}$ CC disappearance measurement with $1^{20}$ POT, MINOS Document 1342 (2006).

[105] S. Kopp et al., Constraining the Beam Neutrino MC Flux Using the MINOS ND Data, MINOS Document 2965 (2008). 
[106] M. Bonesini, A. Marchionni, F. Pietropaolo and T. Tabarelli de Fatis, On particle production for high energy neutrino beams, Eur. Phys. J., C20, 13-27 (2001).

[107] L. Lyons, Statistics for nuclear and particle physicists, Cambridge University Press (1986).

[108] F. James and M. Roos, Minuit: A System for Function Minimization and Analysis of the Parameter Errors and Correlations, Comput. Phys. Commun., 10, 343-367 (1975).

[109] B. W. Silverman, Density Estimation for Statistics and Data Analysis, Chapman \& Hall/CRC (1986).

[110] S. Dytman and H. R. Gallagher, New Intranuke model for Neugen - hA, MINOS Document 3484 (2008).

[111] S. Dytman and H. R. Gallagher, Intranuke Modifications - Pions and Neutrino Comparisons, MINOS Document 3823 (2008).

[112] P. Adamson et al., Measurement of neutrino velocity with the MINOS detectors and NuMI neutrino beam, Phys. Rev., D76, 072005 (2007). 


\section{Vita}

Rustem Ospanov attended high school in Karaganda, Kazakhstan. In 1994 he entered Tomsk State University in Tomsk, Russia, and received the degree of Bachelor of Science in Physics. In the following years he attended the Johns Hopkins University where he received the degree of Master of Arts in Physics. In September, 2002, he entered the physics department at the University of Texas at Austin. He spent most of his graduate career working on the MINOS experiment at the Fermi National Accelerator Laboratory, Batavia, Illinois.

Permanent address: Department of Physics

University of Texas at Austin

1 University Station C1600

Austin, TX 78712-0264

This dissertation was typeset with $\mathrm{LT}_{\mathrm{EX}}^{\dagger}$ by the author.

\footnotetext{
${ }^{\dagger} \mathrm{LTT}_{\mathrm{EX}}$ is a document preparation system developed by Leslie Lamport as a special version of Donald Knuth's TEX Program.
} 